\title{
Clickable Azide-Functionalized Bromo-Aryl-Aldehydes - Synthesis and Photophysical Characterization
}

Dominik Göbel, Marius Friedrich, Enno Lork, Boris Nachtsheim

Submitted date: 04/05/2020 - Posted date: 06/05/2020

Licence: CC BY-NC-ND 4.0

Citation information: Göbel, Dominik; Friedrich, Marius; Lork, Enno; Nachtsheim, Boris (2020): Clickable Azide-Functionalized Bromo-Aryl-Aldehydes - Synthesis and Photophysical Characterization. ChemRxiv. Preprint. https://doi.org/10.26434/chemrxiv.12238967.v1

In this article we describe the functionalization of bromo aryl aldehyde-based fluorophores with azide functionalities, their Cu-catalyzed attachment to alkynes and the effect of these functionalizations on their emission properties.

File list (3)

Manuscript_Chemrxiv_Azides.pdf (491.61 KiB) view on ChemRxiv - download file

Supporting Information_Chemrxiv.pdf (12.33 MiB) view on ChemRxiv - download file 


\section{Clickable Azide-functionalized Bromo-Aryl-}

\section{Aldehydes - Synthesis and Photophysical}

\section{Characterization}

Dominik Göbel ${ }^{1}$, Marius Friedrich ${ }^{1,2}$, Enno Lork ${ }^{3}$, Boris J. Nachtsheim¹

Address: ${ }^{1}$ Institute for Organic and Analytical Chemistry, University of Bremen, Leobener Straße 7, D-28359 Bremen, Germany, ${ }^{2}$ Department of Organic Chemistry, Technical University Kaiserslautern, Erwin-Schrödinger-Straße Geb.54, D-67663 Kaiserslautern, Germany ${ }^{3}$ Institute for Inorganic and Crystallographic Chemistry, University of Bremen, Leobener Straße NW2, D-28359 Bremen, Germany

Email: Boris J. Nachtsheim - nachtsheim@uni-bremen.de

${ }^{*}$ Corresponding author

\section{Abstract}

Herein we present the facile synthesis of three azide-functionalized fluorophores and their covalent attachment as triazoles in Huisgen-type cycloaddition with model alkynes. Besides two ortho- and para-bromo-substituted benzaldehydes, the azide functionalization of a fluorene-based structure will be presented. The copper(I)catalyzed azide-alkyne cycloaddition (CuAAC) of the so synthesized azidefunctionalized bromocarbaldehydes with terminal alkynes exhibiting different degree of steric demand was performed in high efficiency. Finally, we investigated the photophysical properties of the azide-functionalized arenes and their covalently linked 
triazole derivatives to gain deeper insight towards the effect of these covalent linkers on their emission behavior.

\section{Keywords}

fluorenes, bromo-arylaldehydes, click-chemistry, phosphorescence, fluorescence

\section{Introduction}

Small organic luminophores exhibiting room-temperature phosphorescence (RTP) have attracted great attention due to promising applications in optoelectronic devices [1-8], biological imaging [9-12] and chemical sensing [13-15]. Referring to the Jablonski diagram (see Scheme 1a) $[16,17]$, upon excitation from the singlet ground state $\left(S_{0}\right)$ to higher singlet states $\left(S_{n}\right)$, followed by internal conversion (IC), either nonradiative or radiative decay to $S_{0}$ can occur. While the latter decay (fluorescence) takes place without change in electron spin, phosphorescence is defined as the radiative transition from the lowest excited triplet state $\left(T_{1}\right)$ to the singlet ground state $\left(\mathrm{S}_{0}\right)$ [1821]. Triplet state excitons are generated by the spin-forbidden intersystem crossing (ISC) process from the first excited singlet state $\left(\mathrm{S}_{1}\right)$. Pursuant to the El-Sayed rule (see Scheme 1b) [22, 23], ISC is spin allowed from ${ }^{1}\left(n, \pi^{*}\right)$ to ${ }^{3}\left(\pi, \pi^{*}\right)$ and from ${ }^{1}(\pi$, $\left.\pi^{*}\right)$ to ${ }^{3}\left(n, \pi^{*}\right)$ excited states, while ISC is spin-forbidden from ${ }^{1}\left(n, \pi^{*}\right)$ to ${ }^{3}\left(n, \pi^{*}\right)$ and from ${ }^{1}\left(\pi, \pi^{*}\right)$ to ${ }^{3}\left(\pi, \pi^{*}\right)$ excited states, owing to poor orbital overlap, resulting in decreased spin-orbit coupling. 
a)

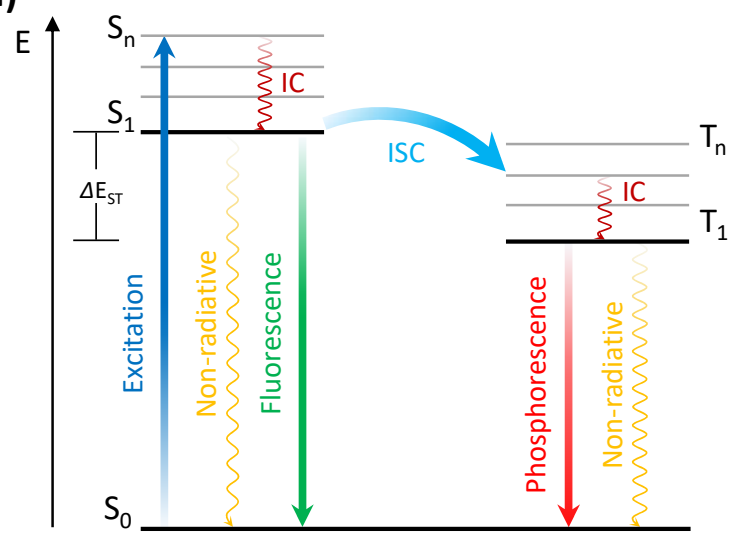

b)

\section{El-Sayed Rule}

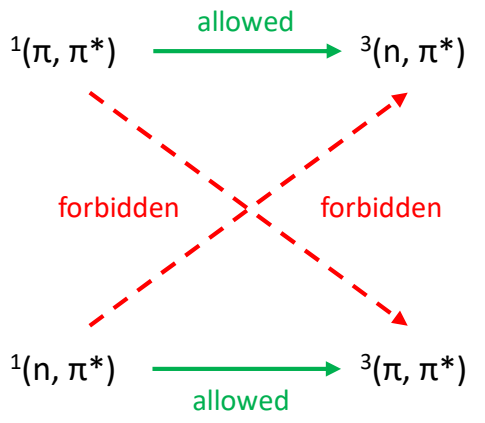

Scheme 1: a) Schematic depiction of the Jablonski diagram; b) Schematic representation of El-Sayed's rule.

Quenching processes of triplet states, induced by molecular motions, oxygen or humidity, restrict the versatile application of such organic materials [24]. Thus, low temperatures [25-27] or inert conditions [28] are necessary to facilitate afterglow emission.

Most phosphorescence studies are focused on metal complexes, due to a strong heavy atom-induced spin-orbit coupling [29-33]. Considering the high price and potential of toxicity of many metal-complexes, pure organic phosphors are highly desirable $[16,17$, 34-37]. Two approaches are applied to achieve organic phosphors. (1) Introduction of non-metal heavy atoms such as halogens $(\mathrm{Br}$ or $\mathrm{I})$ or functionalities containing lone pairs, in particular carbonyls, nitrogen, sulfur and phosphorus derivatives which 
facilitate the ISC process from $\mathrm{S}_{1}$ to $\mathrm{T}_{\mathrm{n}}$ and thus increase the spin-orbital coupling [3847]. Also, decreasing the singlet-triplet splitting energy ( $\Delta \mathrm{E} s \mathrm{~s})$ caused by intramolecular charge-transfer (ICT) interactions is an approved method [48, 49]. (2) Significant reduction of nonradiative transitions can be achieved by the host-guest method [5052] or by crystallization [53-57]. In difference to liquid phase, the highly ordered packing and the restriction of molecular motions in the crystalline state favor persistent luminescence.

The promotion of ISC processes through intermolecular halogen bonding to generate efficient RTP was initially investigated by Kim et al. [58]. They developed the minimalistic 2,5-dihexyloxy-4-bromobenzaldehyde 1 [59-63] which shows weak fluorescence in solution, but exhibits green phosphorescence with a quantum yield of $\Phi_{\mathrm{P}}=2.9 \%$ in the crystalline state: This behavior is caused by intermolecular halogen bonds from the carbonyl-oxygen to an adjacent bromine-atom (Figure 1a).

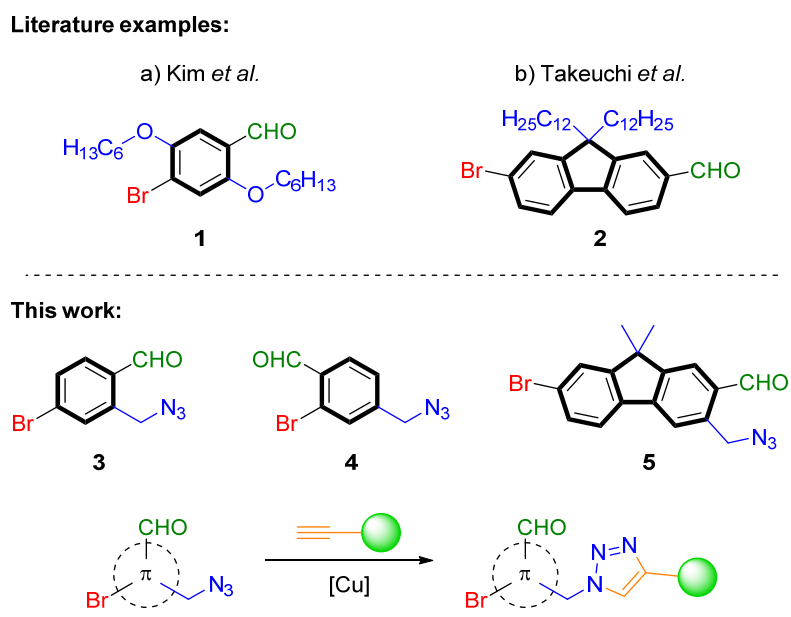

Figure 1: Top: Literature examples of organic compounds showing RTP in the crystalline state (a) and in solution (b). Bottom: Azide-linked derivatives presented in this work.

Despite multifarious examples of RTP in the crystalline state, purely organic compounds showing RTP in solution are rare [64-69]. Takeuchi et al. [28] reported a 
bromofluorenecarbaldehyde 2 which shows blue fluorescence in chloroform at $298 \mathrm{~K}$ under air and green phosphorescence under argon with a phosphorescence quantum yield of $\Phi_{P}=5.9 \%$ (Figure $1 \mathrm{~b}$ ). This observation is reasoned by a strong $\left(\pi, \pi^{*}\right)$ character of the $\mathrm{T}_{2}$-state.

Although phosphorescent organic compounds are well investigated towards their photophysical properties in the crystalline state, in solution or physically embedded in polymer matrices, there is a significant lack of possibilities [61] for their targeted covalent attachment to higher structures. This is due to missing synthetic strategies to incorporate suitable linkable functionalities into those luminophores.

Our group is highly interested in the de novo synthesis of small organic luminophores and in this regard we recently developed efficient methods for the synthesis of ESIPTbased luminophores $[70,71]$. Herein, we present the efficient functionalization of derivatives of the potent luminophores $\mathbf{1}$ and $\mathbf{2}$ with "clickable" azide functionalities to target structures 3-5 and further investigated the influence of this functionalization, both in the unlinked azide state and the linked triazole state, on the emission properties of these compounds (Figure 1).

\section{Results and Discussion}

\section{Syntheses}

\section{para- and ortho-Bromobenzaldehyde 3 and 4}

We initiated our synthetic investigations towards azide-functionalized parabromobenzaldehyde $\mathbf{3}$ in a two-step sequence. Condensation with 2-amino-2methylpropan-1-ol and oxidation with NBS yielded the oxazoline 6 in good yield. Directed ortho-metalation utilizing $\mathrm{TMPMgCl} \cdot \mathrm{LiCl}$ under mild conditions and subsequent smooth formylation with DMF afforded benzaldehyde 7. Due to rapid 
decomposition of 7 under ambient and acidic conditions, rapid aqueous work-up was conducted, followed by reduction with $\mathrm{NaBH}_{4}$, yielding the corresponding primary alcohol 8 in $81 \%$ over two steps. The transformation to azide 9 was accomplished by deprotonation using 1,8-diazabicyclo[5.4.0]undec-7-ene (DBU) and reaction with diphenylphosphoryl azide (DPPA) in excellent yield. Finally, the oxazoline group, which acted as directing and protecting group, was removed in a three-step sequence of $N$-methylation, reduction of the in situ formed iminium ion and acidic hydrolysis. This afforded the azide-functionalized para-bromobenzaldehyde 3 in $78 \%$ yield and an overall yield of $56 \%$ (starting from 4-bromobenzaldehyde).

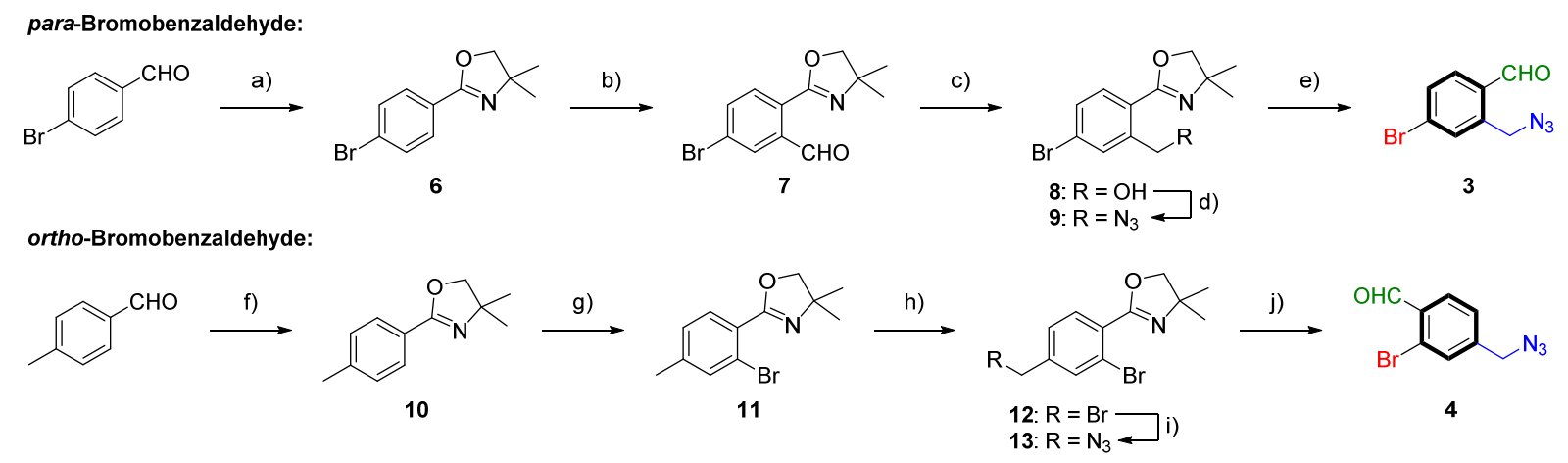

Scheme 2: Reaction conditions: para-Bromobenzaldehyde 3: a) 1) 2-amino-2methylpropan-1-ol, $4 \AA \mathrm{MS}, \mathrm{CH}_{2} \mathrm{Cl}_{2}, 25^{\circ} \mathrm{C}, 18 \mathrm{~h}$; 2) $\mathrm{NBS}, \mathrm{CH}_{2} \mathrm{Cl}_{2}, 25^{\circ} \mathrm{C}, 5 \mathrm{~h}, 91 \%$; b) 1) $\mathrm{TMPMgCl} \cdot \mathrm{LiCl}, \mathrm{THF}, 25{ }^{\circ} \mathrm{C}, 4 \mathrm{~h} ; 2$ ) DMF, THF, $0{ }^{\circ} \mathrm{C}$ to $25{ }^{\circ} \mathrm{C}, 1.5 \mathrm{~h}$; c) $\mathrm{NaBH}_{4}$, THF:MeOH 1:1, $0{ }^{\circ} \mathrm{C}, 1$ h, $81 \%$ (2 steps); d) DPPA, DBU, PhMe, $25{ }^{\circ} \mathrm{C}, 18$ h, $98 \%$; e) 1) MeOTf, $\mathrm{CH}_{2} \mathrm{Cl}_{2}, 25^{\circ} \mathrm{C}, 2.5 \mathrm{~h} ; 2$ ) $\mathrm{NaBH}_{4}$, THF:MeOH $\left.4: 1 \mathrm{v}: \mathrm{v}, 0{ }^{\circ} \mathrm{C}, 2.5 \mathrm{~h} ; 3\right)$ oxalic acid, THF: $\mathrm{H}_{2} \mathrm{O} 4: 1 \mathrm{v}: \mathrm{v}, 25^{\circ} \mathrm{C}, 20 \mathrm{~h}, 78 \%$. Overall yield from 4-bromobenzaldehyde to 3: $56 \%$ (5 steps). ortho-Bromobenzaldehyde 4: f) 1) 2-amino-2-methylpropan-1-ol, 4 $\AA \mathrm{MS}, \mathrm{CH}_{2} \mathrm{Cl}_{2}, 25^{\circ} \mathrm{C}, 18 \mathrm{~h} ;$ 2) NBS, $\mathrm{CH}_{2} \mathrm{Cl}_{2}, 25^{\circ} \mathrm{C}, 4 \mathrm{~h}, 98 \%$; g) 1) TMPMgCl.LiCl, THF, $\left.25{ }^{\circ} \mathrm{C}, 4 \mathrm{~h} ; 2\right)\left(\mathrm{CBrCl}_{2}\right) 2, \mathrm{THF}, 0{ }^{\circ} \mathrm{C}$ to $25^{\circ} \mathrm{C}, 10 \mathrm{~h}, 76 \%$; h) NBS, AIBN, $\mathrm{CCl}_{4}$, $100{ }^{\circ} \mathrm{C}, 7 \mathrm{~h}, 66 \%$; i) $\mathrm{NaN}_{3}, \mathrm{DMF}, 25^{\circ} \mathrm{C}, 4 \mathrm{~h}, 99 \%$; j) 1) $\mathrm{MeOTf} \mathrm{CH}_{2} \mathrm{Cl}_{2}, 25^{\circ} \mathrm{C}, 2.5 \mathrm{~h}$; 2) $\mathrm{NaBH}_{4}$, THF:MeOH 4:1 v:v, $0{ }^{\circ} \mathrm{C}, 2.5 \mathrm{~h}$; 3) oxalic acid, THF: $\mathrm{H}_{2} \mathrm{O} 4: 1 \mathrm{v}: \mathrm{v}, 25^{\circ} \mathrm{C}, 20 \mathrm{~h}$, 85\%. Overall yield from 4-methylbenzaldehyde to 4: 41\% (5 steps). 
Azide-functionalized ortho-bromobenzaldehyde 4 was prepared by a similar route as aldehyde 3. Initially, oxazoline formation from 4-methylbenzaldehyde yielded 2aryloxazoline $\mathbf{1 0}$ in almost quantitative yield. Introduction of the ortho-bromine substituent was again accomplished by metalation using $\mathrm{TMPMgCl} \cdot \mathrm{LiCl}$ and subsequent reaction with 1,2-dibromotetrachloroethane to afford $\mathbf{1 1}$ in $76 \%$ yield. A second bromination at benzylic position provided the dibrominated derivative 12 in $66 \%$ yield. Substitution reaction of benzyl bromide with sodium azide delivered primary azide 13 in quantitative yield. Again, final back-conversion of the oxazoline group to the corresponding aldehyde afforded azide-functionalized ortho-bromobenzaldehyde 4 in $85 \%$ yield and an overall yield of $41 \%$ (starting from 4-methylbenzaldehyde).

\section{Bromofluorenecarbaldehyde 5}

The synthetic route to azide-functionalized 7-bromofluorene-2-carbaldehyde 5 started from unfunctionalized fluorene. Double bromination to 14 , followed by double methylation of the methylene bridge to 15 and a lithiation/formylation sequence afforded 7-bromofluorene-2-carbaldehyde 16 in excellent yield over three steps. Conversion to the 2-aryloxazoline 17 was accomplished in $92 \%$ yield using the same method as described for the synthesis of 6 and 10. ortho-Metalation with TMPMgCl-LiCl [70] and conversion of the magnesiated species with DMF to carbaldehyde 18 was followed by reduction with $\mathrm{NaBH}_{4}$ to give the primary alcohol 19 . In contrast to benzaldehyde 7, carbaldehyde $\mathbf{1 8}$ showed no decomposition at ambient temperature. While acidic hydrolysis of 19 provided exocyclic $y$-lactone $\mathbf{2 0}$, substitution reaction with DPPA/NaN 3 yielded the primary azide in $87 \%$ yield. In accordance to previous deprotection reactions, fluorene $\mathbf{2 1}$ was converted by means of a three-step sequence to the desired azide-functionalized 7-bromofluorene-2-carbaldehyde $\mathbf{5}$ in $86 \%$ yield and an overall yield of $45 \%$ (starting from fluorene). The molecular structure of 5 could be verified by X-ray diffraction (XRD, see Scheme 3). 


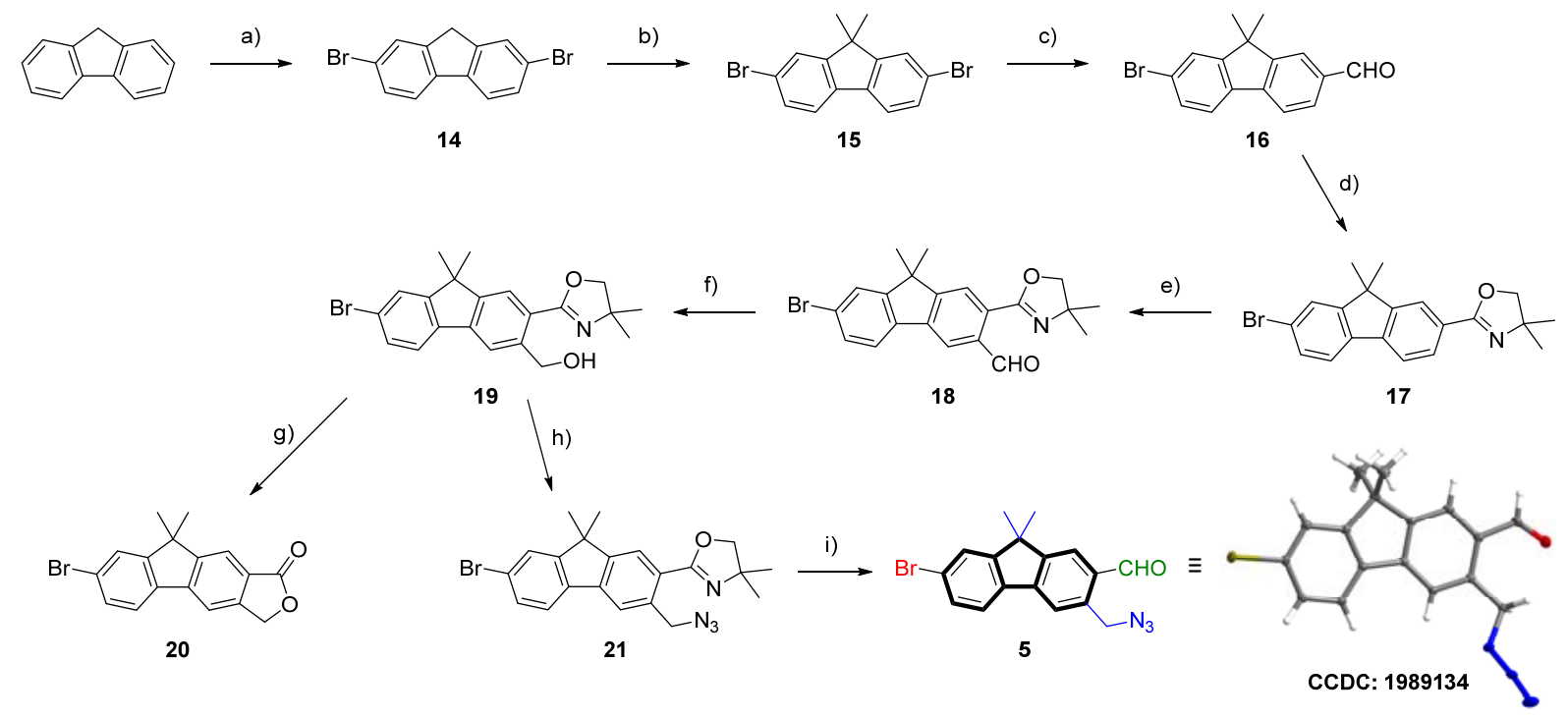

Scheme 3: Reaction conditions: a) $\mathrm{Br}_{2}$, Fe-powder, $\mathrm{CHCl}_{3}, \mathrm{O}^{\circ} \mathrm{C}, 4 \mathrm{~h}, 99 \%$; b) $\mathrm{KOH}$, $\mathrm{KI}, \mathrm{Mel}, \mathrm{DMSO}, 25^{\circ} \mathrm{C}, 18 \mathrm{~h}, 92 \%$; c) 1) $n \mathrm{BuLi}, \mathrm{THF},-78^{\circ} \mathrm{C}, 1 \mathrm{~h}$; 2) DMF, THF, $-78^{\circ} \mathrm{C}$ to $25{ }^{\circ} \mathrm{C}, 10 \mathrm{~h}, 90 \%$; d) 1) 2-amino-2-methylpropan-1-ol, $4 \AA \mathrm{MS}, \mathrm{CH}_{2} \mathrm{Cl}_{2}, 25^{\circ} \mathrm{C}, 18 \mathrm{~h}$; 2) NBS, $\mathrm{CH}_{2} \mathrm{Cl}_{2}, 25^{\circ} \mathrm{C}, 3 \mathrm{~h}, 92 \%$; e) 1) TMPMgCl$\left.\cdot \mathrm{LiCl}, \mathrm{THF}, 25^{\circ} \mathrm{C}, 4 \mathrm{~h} ; 2\right) \mathrm{DMF}, \mathrm{THF}$, $-15{ }^{\circ} \mathrm{C}$ to $25^{\circ} \mathrm{C}, 2 \mathrm{~h}$; f) $\mathrm{NaBH}_{4}$, THF:MeOH 1:1 v:v, $0{ }^{\circ} \mathrm{C}, 1.5 \mathrm{~h}, 79 \%$ (2 steps); g) $\mathrm{HCl}$ $(4 \mathrm{~N}), 120^{\circ} \mathrm{C}, 6 \mathrm{~h}, 91 \%$; h) 1) DPPA, DBU, PhMe, $\left.25^{\circ} \mathrm{C}, 15 \mathrm{~h} ; 2\right) \mathrm{NaN}_{3}, \mathrm{PhMe}, 60{ }^{\circ} \mathrm{C}$, $4 \mathrm{~h}, 87 \%$; i) 1) MeOTf, $\mathrm{CH}_{2} \mathrm{Cl}_{2}, 25^{\circ} \mathrm{C}, 2.5 \mathrm{~h}$; 2) $\mathrm{NaBH}_{4}$, THF:MeOH 4:1 v:v, $0{ }^{\circ} \mathrm{C}, 2.5 \mathrm{~h}$; 3) oxalic acid, THF: $\mathrm{H}_{2} \mathrm{O} 4: 1 \mathrm{v}: \mathrm{v}, 25^{\circ} \mathrm{C}, 20 \mathrm{~h}, 86 \%$. Overall yield to 5: $45 \%$ (8 steps). The molecular structure of $\mathbf{5}$ shows $50 \%$ probability ellipsoids.

\section{Derivatization of fluorenyl methanol 19}

To gain deeper insights about the emission behavior of fluorenes bearing different functional groups in the side chains, fluorenyl methanol 19 was subjected to derivatization reactions. Deprotonation and subsequent methylation afforded methoxy derivative 22, which was then converted into the methoxy-functionalized 7-bromofluorene-2-carbaldehyde 23 in 75\% yield. Implementation of potent leaving groups in the side chain as complement linkable functionalities via $\mathrm{S}_{\mathrm{N}}$-reaction was 
initiated by mesylation. Unfortunately, the mesylated fluorene showed such a high reactivity that rapid decomposition occurred. However, bromination was conducted by various substitution methods delivering benzyl bromide $\mathbf{2 4}$, which upon isolation cyclized to iminium bromide 25 in high yields. To suppress this unexpected cyclization, careful fine-adjustment of the work-up conditions were made. Here, upon complete formation of 24, rapid filtration of the reaction mixture through a plug of neutral alumina, solvent evaporation and quick conversion in the next step was successfully applied. Deprotection to the carbaldehyde was performed using the well applied three-step methylation/reduction/hydrolysis sequence. Methylation of the oxazoline nitrogen afforded iminium salt 26, which was reduced to oxazolidine 27 . Again, similar to $\mathbf{2 5}$, cyclization took place and ammonium triflate $\mathbf{2 8}$ was isolated in $56 \%$ yield (starting from 24). In contrast to the cyclization of oxazoline 24, oxazolidine 27 cyclized already during the reaction, caused by the increased basicity of the ring nitrogen.

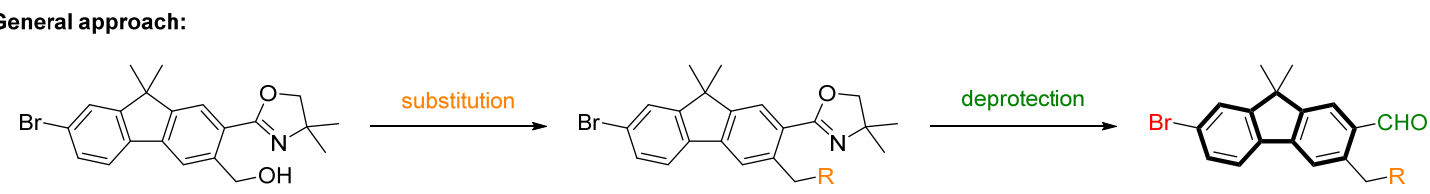

19

Derivatization:
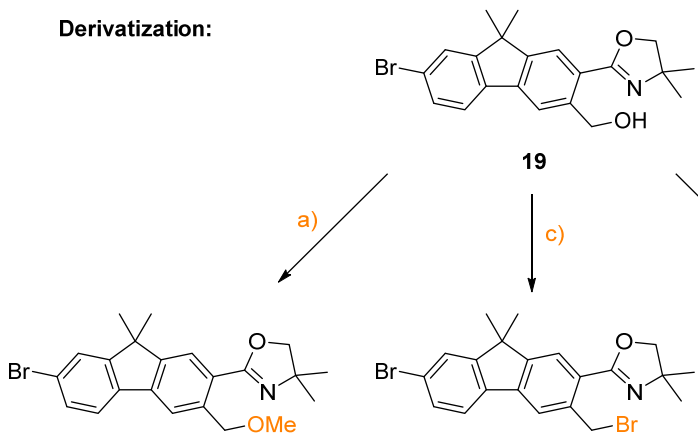

22
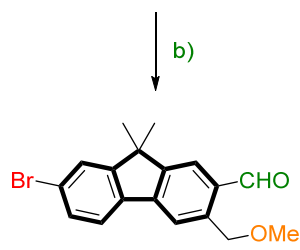

23
19

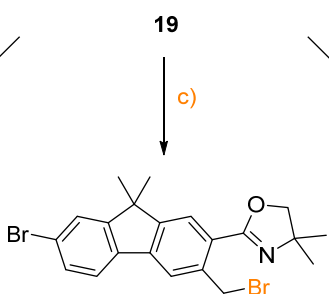

24

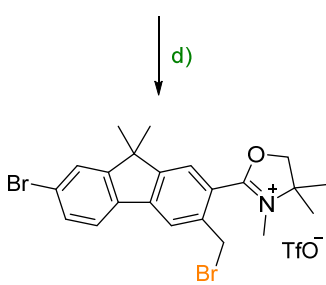

26

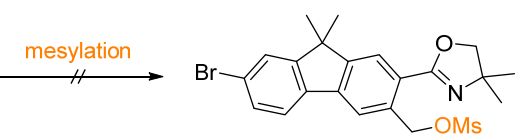

unstable

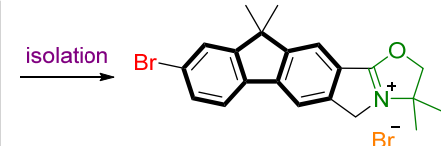

25

24

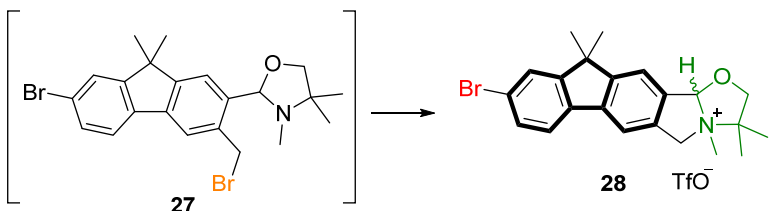


Scheme 4: Reaction conditions: a) 1) $\mathrm{NaH}, \mathrm{THF}, 0{ }^{\circ} \mathrm{C}, 30 \mathrm{~min}$; 2) Mel, THF, $0{ }^{\circ} \mathrm{C}$ to $25{ }^{\circ} \mathrm{C}, 2 \mathrm{~h}, 99 \%$; b) 1) MeOTf, $\mathrm{CH}_{2} \mathrm{Cl}_{2}, 25^{\circ} \mathrm{C}, 3 \mathrm{~h}$; 2) $\mathrm{NaBH}_{4}$, THF:MeOH 4:1 v:v, $0{ }^{\circ} \mathrm{C}$, $3 \mathrm{~h}$; 3) oxalic acid, THF: $\mathrm{H}_{2} \mathrm{O} 4: 1 \mathrm{v}: \mathrm{v}, 25^{\circ} \mathrm{C}, 24 \mathrm{~h}, 75 \%$. Overall yield from fluorene to 23: $44 \%$ (8 steps). c) $\mathrm{CBr}_{4}, \mathrm{PPh}_{3}, \mathrm{CH}_{2} \mathrm{Cl}_{2}, 0{ }^{\circ} \mathrm{C}$ to $25^{\circ} \mathrm{C}, 2 \mathrm{~h}, 95 \%$; or $\mathrm{NBS}, \mathrm{PPh}_{3}$, $\mathrm{CH}_{2} \mathrm{Cl}_{2}, 0{ }^{\circ} \mathrm{C}$ to $25^{\circ} \mathrm{C}, 2 \mathrm{~h}, 91 \%$; or $\mathrm{PBr}_{3}, \mathrm{CH}_{2} \mathrm{Cl}_{2}, 0{ }^{\circ} \mathrm{C}$ to $25^{\circ} \mathrm{C}, 2 \mathrm{~h}, 92 \%$; d) MeOTf, $\mathrm{CH}_{2} \mathrm{Cl}_{2}, 25^{\circ} \mathrm{C}, 3 \mathrm{~h}$; e) $\mathrm{NaBH}_{4}$, THF:MeOH 4:1 v:v, $0{ }^{\circ} \mathrm{C}, 3 \mathrm{~h}$. Overall yield from 24 to 28: $56 \%$ (2 steps).

\section{CuAAC reactions of bromocarbaldehydes}

We further investigated the reactivity of azide-functionalized bromocarbaldehydes 3, 4 and $\mathbf{5}$ in copper(I)-catalyzed azide-alkyne cycloaddition reactions (CuAAC). For this, we treated the azide-functionalized luminophores with alkynes exhibiting different degree of steric demand, including 1-decyne 29, phenyl acetylene $\mathbf{3 0}$, 1-ethynyladamantane 31 and 1,3-di-tert-butyl-5-ethynylbenzene 32 (see Scheme 5). All triazoles 33-44, based on the bromocarbaldehydes 3, 4 and 5 were successfully isolated in excellent yields of $>90 \%$. As a further model functionalization, the sterically demanding adamantly substituted triazole $\mathbf{4 2}$ was subjected to a methylation reaction with Meerwein's salt (trimethyloxonium tetrafluoroborate) to deliver the $\mathrm{N}$-methylated triazole 45 in $83 \%$ yield. 


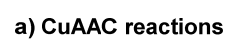

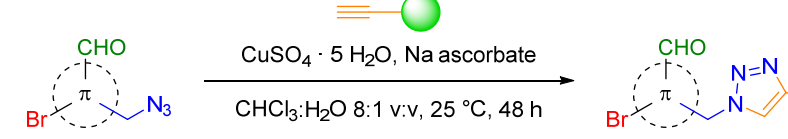
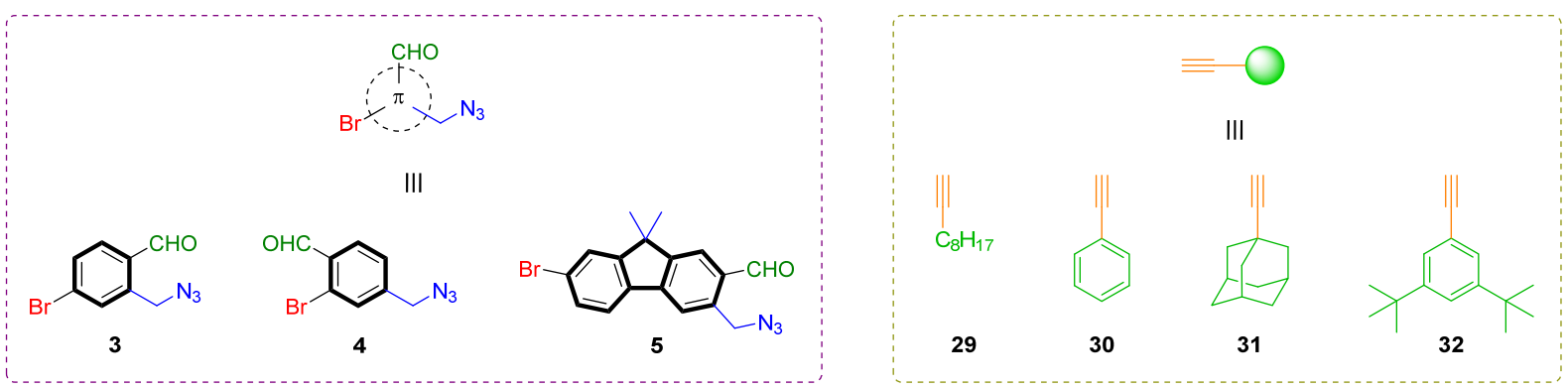

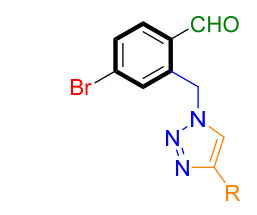

33: $\mathrm{R}=$ octyl $(94 \%)$

34: $R=$ adamantyl $(97 \%)$

35: $R=$ phenyl $(99 \%)$

36: $R=$ bis $(t \mathrm{Bu})$ Phenyl $(96 \%)$

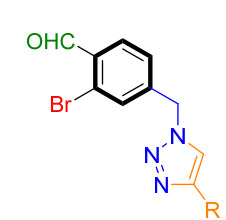

37: $\mathrm{R}=$ octyl $(92 \%)$

38: $R=$ adamantyl $(97 \%)$

39: $R=$ phenyl $(97 \%)$

40: $R=$ bis(tBu)Phenyl $(94 \%)$

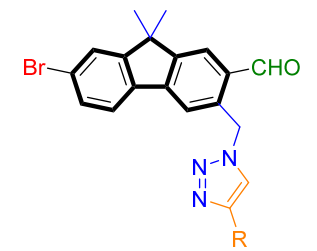

41: $R=$ octyl $(91 \%)$

42: $R=$ adamantyl $(99 \%)$

43: $R=$ phenyl $(94 \%)$

44: $\mathrm{R}=$ bis $(t \mathrm{Bu}) \mathrm{Phenyl}(98 \%)$

b) Methylation reaction
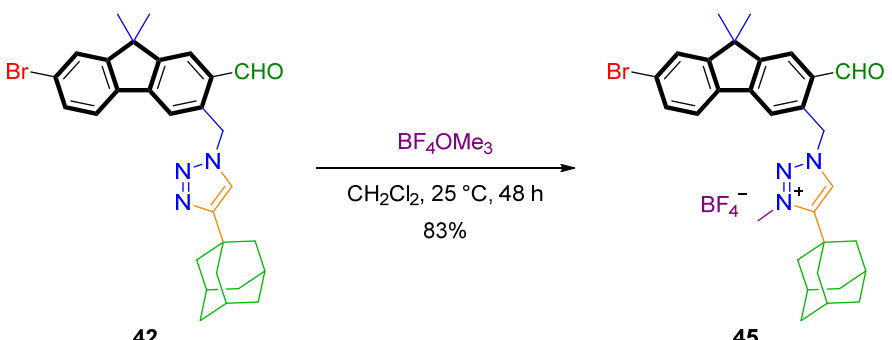

45

Scheme 5: a) CuAAC reactions of azide-functionalized bromocarbaldehydes 3, 4 and 5 with terminal alkynes to triazoles 33-44. General reaction conditions for CuAAC reactions: Azide (1.00 eq), terminal alkyne (1.05 eq), $\mathrm{CuSO}_{4} \cdot 5 \mathrm{H}_{2} \mathrm{O}(0.1 \mathrm{eq}), \mathrm{Na}$ ascorbate $(0.50 \mathrm{eq}), \mathrm{CHCl}_{3},(0.1 \mathrm{M})$ and water $(12.5 \mathrm{mM})$ at $25{ }^{\circ} \mathrm{C}$ for $48 \mathrm{~h}$. b) Methylation reaction of adamantly substituted triazole 42 with Meerwein's salt (trimethyloxonium tetrafluoroborate).

\section{Photophysical properties}

We finally examined the photophysical properties of both the azides and the triazoles.

UV-Vis absorption measurements of para-Bromobenzaldehyde 3 and ortho- 
Bromobenzaldehyde 4 as well as the corresponding triazoles $33-40$ were conducted in chloroform. Intense absorption bands below $270 \mathrm{~nm}$ are observed, which can be attributed to typical $\pi-\Pi^{*}$ transitions deriving from the single-benzene core. In addition, unstructured absorption bands above $290 \mathrm{~nm}$ are observed, while ortho-derivatives exhibit more broadened bands than the para-derivatives (see Figure 2a and S1-S11). Emission spectra for para-bromocarbaldehyde $\mathbf{3}$ and adamantyl-triazole $\mathbf{3 4}$ in the solid-state show maxima at $440 \mathrm{~nm}$ (for compound 3) and $416 \mathrm{~nm}$ (for compound 34) (see Figure 2b). Additionally, fluorescence half lifetimes $(T)$ of $\mathbf{3}$ and $\mathbf{3 4}$ were determined by time correlated single photon counting (TCSPC), indicating low halflifetimes of 2.21 ns (for compound 3) and 3.23 ns (for compound 34) for lower populated species (see Figure S6). Quantum yields $(\Phi)$ of both derivatives are below $1 \%$ in the solid-state. In chloroform no luminescence was detected for the paraderivatives. All ortho-derivatives exhibit no luminescence neither in solution nor in the solid-state [72].
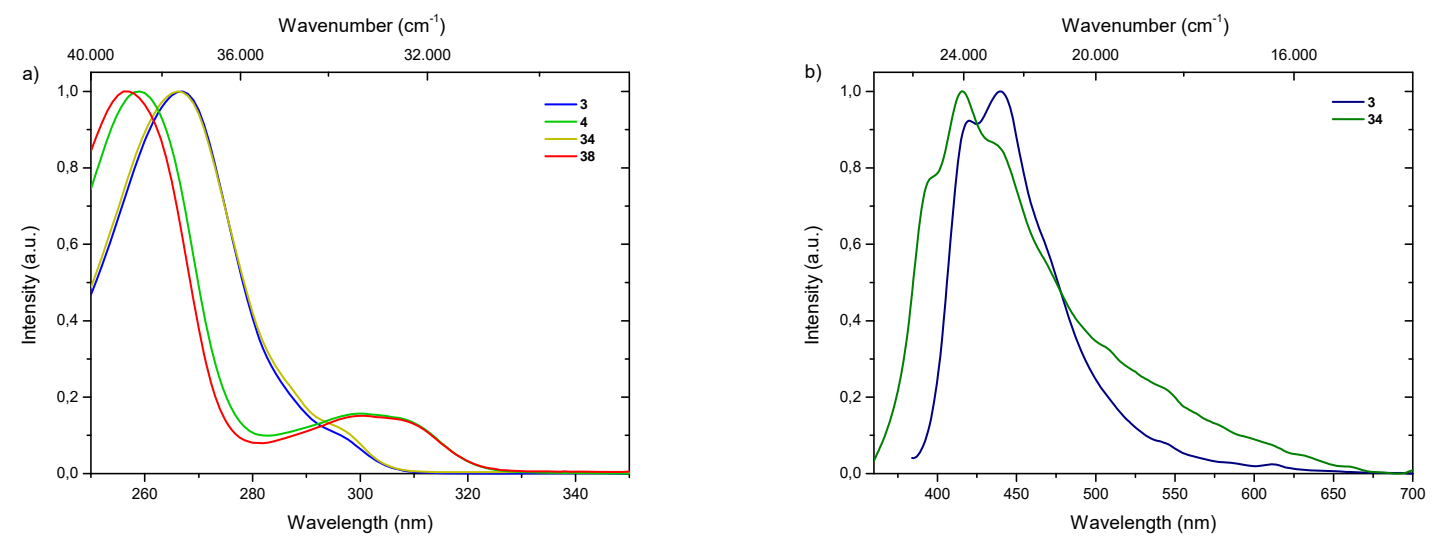

Figure 2: a) Normalized UV-Vis absorption spectra of $\mathbf{3}$ (blue line), $\mathbf{3 4}$ (green line), $\mathbf{4}$ (yellow line) and $\mathbf{3 8}$ (red line) in $\mathrm{CHCl}_{3}$. b) Normalized emission spectra of $\mathbf{3}$ (navy line), 34 (olive line) in $\mathrm{CHCl}_{3}$ after bubbling with argon for 30 minutes. 
These observations for para- and ortho-bromobenzaldehydes indicate that phosphorescence is quenched in these systems yielding only weak fluorescence. This may be owing to the azide-moiety either as functional group or as part of the triazole heterocycle. Similar observations were already made on luminescent materials [73, 74].

Fluorene derivatives were subjected to photophysical measurements as well. UV-Vis absorption spectra of bromofluorenecarbaldehydes 5 and $\mathbf{1 6}$ show intense absorption bands at 331 and $330 \mathrm{~nm}$. Furthermore, adamantyl-triazole 42 and the corresponding tetrafluoroborate salt $\mathbf{4 5}$ exhibit similar absorption properties, while the methylated species 45 shows a comparable slightly red-shifted absorption band (see Figure 3a and S12-S14). Emission measurements of carbaldehydes 5 and 16 reveal that solidstate emission bands are more blue-shifted than emission bands in solution (see Figure 3b). Similar observations were made for adamantyl-triazole 42 and tetrafluoroborate salt 45 with maxima at $510 \mathrm{~nm}$ and $540 \mathrm{~nm}$ (see Figure 3c). However, TCSPC demonstrate that solely fluorescence is observed in all physical states for all investigated compounds with a maximum half-lifetime $\tau$ of $11.6 \mathrm{~ns}$ for methylated triazole 45 in chloroform (see Figure 3d). Quantum yields $\Phi$ were determined to be $<1 \%$ for all structures. Further fluorene derivatives - methoxymethyl carbaldehyde 23, iminium bromide 25 and ammonium triflate 28 - exhibit intense absorption maxima around $320 \mathrm{~nm}$ (see Figure S13). For 25 and 28, emission maxima in the solid-state were red-shifted compared to emission bands in chloroform solution (see Figure S31 and S33). 23 Exhibits a deep violet emission maximum at $360 \mathrm{~nm}$ in chloroform solution and no emission in the solid-state (see Figure S30). Half-lifetimes $T$ were defined up to 8.66 ns for iminium bromide 25 , with quantum yields $\Phi$ below $1 \%$ (see Figure S32). The absence of any long living triplet species in all fluorene derivatives in 
solution as well as in the solid-state again indicates undesirable quenching events induced by the azide functionalities, similar to para- and ortho-bromobenzaldehydes.
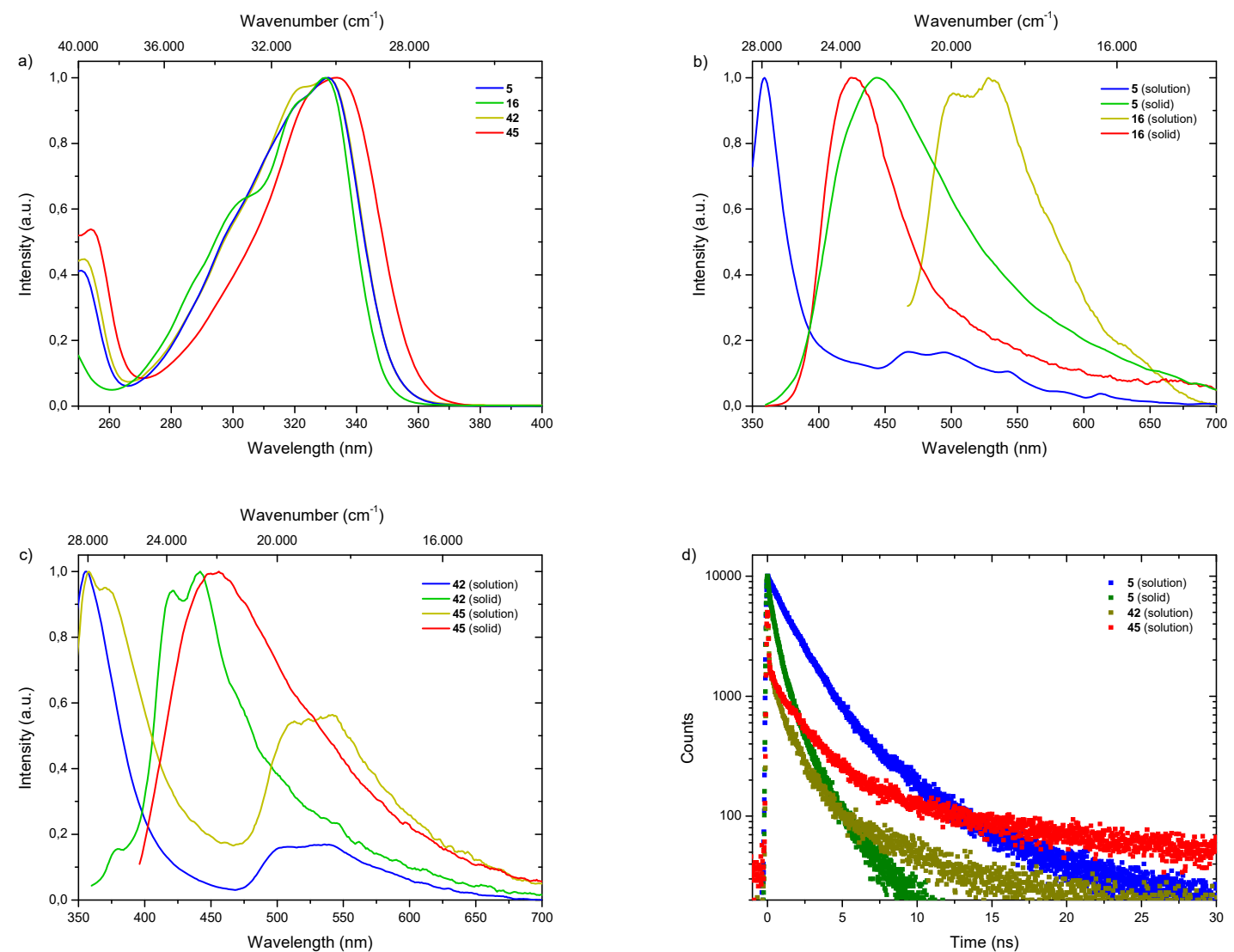

Figure 3: a) Normalized UV-Vis absorption spectra of 5 (blue line), 16 (green line), 42 (yellow line) and $\mathbf{4 5}$ (red line) in $\mathrm{CHCl}_{3}$. b) Normalized emission spectra of $\mathbf{5}$ (in solution, blue line and solid-state, green line) and $\mathbf{1 6}$ (in solution, yellow line and solid-state, red line). c) Normalized emission spectra of $\mathbf{4 2}$ (in solution, blue line and solid-state, green line) and 45 (in solution, yellow line and solid-state, red line). d) Time resolved emission decay curves of $\mathbf{5}$ (in solution, blue dots and solid-state, green dots), $\mathbf{4 2}$ (in solution, yellow dots) and 45 (in solution, red dots) at $25^{\circ} \mathrm{C}$. In all diluted measurements $(c=$ $\left.10^{-5} \mathrm{~mol} \cdot \mathrm{L}^{-1}\right) \mathrm{CHCl}_{3}$ was used as solvent and solutions were sparged with argon for 30 minutes. 


\section{Conclusion}

In summary, azide-functionalized ortho- and para-bromo benzaldehydes and fluorene derivates were successfully synthesized. The azide bearing arenes were efficiently linked with even sterically highly demanding alkynes in a copper(I)-catalyzed azidealkyne cycloaddition (CuAAC). Initial photophysical investigations of azidefunctionalized bromocarbaldehydes and fluorenes revealed that the azide-moiety and the triazole heterocycle efficiently quench phosphorescent transitions. Efforts of installing other functional groups suitable for covalent connections or modification of the alkyl bridge between the arene and the azide are underway in our laboratories.

\section{Supporting Information}

Supporting Information File 1:

Detailed experimental procedures, characterization data, photophysical properties, and copies of NMR spectra.

Supporting Information File 2:

Crystallographic data for compound $\mathbf{5}$.

\section{Acknowledgements}

We thank Prof. Dr. Nadja-C. Bigall, Dr. Dirk Dorfs and Pascal Rusch (all from Leibniz University Hannover) for supporting the photophysical measurements.

\section{References}

1. Kabe, R.; Notsuka, N.; Yoshida, K.; Adachi, C. Adv. Mater. 2016, 28, 655-660. doi:10.1002/adma.201504321 
2. Tao, Y.; Yang, C.; Qin, J. Chem. Soc. Rev. 2011, 40, 2943-2970. doi:10.1039/c0cs00160k

3. Goushi, K.; Yoshida, K.; Sato, K.; Adachi, C. Nat. Photonics 2012, 6, 253-258. doi:10.1038/nphoton.2012.31

4. Zhang, Q.; Li, B.; Huang, S.; Nomura, H.; Tanaka, H.; Adachi, C. Nat. Photonics 2014, 8, 326-332. doi:10.1038/nphoton.2014.12

5. Chaudhuri, D.; Wettach, H.; van Schooten, K. J.; Liu, S.; Sigmund, E.; Höger, S.; Lupton, J. M. Angew. Chem. Int. Ed. 2010, 49, 7714-7717. doi:10.1002/anie.201003291

6. Chaudhuri, D.; Sigmund, E.; Meyer, A.; Röck, L.; Klemm, P.; Lautenschlager, S.; Schmid, A.; Yost, S. R.; van Voorhis, T.; Bange, S.; Höger, S.; Lupton, J. M. Angew. Chem. Int. Ed. 2013, 52, 13449 13452. doi:10.1002/anie.201307601

7. Murawski, C.; Leo, K.; Gather, M. C. Adv. Mater. 2013, 25, 6801-6827. doi:10.1002/adma.201301603

8. Baldo, M. A.; O'Brien, D. F.; You, Y.; Shoustikov, A.; Sibley, S.; Thompson, M. E.; Forrest, S. R. Nature 1998, 395, 151-154. doi:10.1038/25954

9. Gao, R.; Mei, X.; Yan, D.; Liang, R.; Wei, M. Nat. Commun. 2018, 9, 2798. doi:10.1038/s41467018-05223-3

10. Miao, Q.; Xie, C.; Zhen, X.; Lyu, Y.; Duan, H.; Liu, X.; Jokerst, J. V.; Pu, K. Nat. Biotechnol. 2017, 35, 1102-1110. doi:10.1038/nbt.3987

11. Zhen, X.; Tao, Y.; An, Z.; Chen, P.; Xu, C.; Chen, R.; Huang, W.; Pu, K. Adv. Mater. 2017, 29, 1606665. doi:10.1002/adma.201606665

12. Zhang, G.; Palmer, G. M.; Dewhirst, M. W.; Fraser, C. L. Nat. Mater. 2009, 8, 747-751. doi:10.1038/nmat2509

13. DeRosa, C. A.; Seaman, S. A.; Mathew, A. S.; Gorick, C. M.; Fan, Z.; Demas, J. N.; Peirce, S. M.; Fraser, C. L. ACS Sens. 2016, 1, 1366-1373. doi:10.1021/acssensors.6b00533

14. Lehner, P.; Staudinger, C.; Borisov, S. M.; Klimant, I. Nat. Commun. 2014, 5, 4460. doi: $10.1038 /$ ncomms 5460

15. Kwon, M. S.; Lee, D.; Seo, S.; Jung, J.; Kim, J. Angew. Chem. Int. Ed. 2014, 53, 11177-11181. doi:10.1002/anie. 201404490 
16. Xiao, L.; Fu, H. Chem. Eur. J. 2019, 25, 714-723. doi:10.1002/chem.201802819

17. Jia, W.; Wang, Q.; Shi, H.; An, Z.; Huang, W. Chem. Eur. J. 2020, 26, 4437-4448. doi:10.1002/chem.201904500

18. Kasha, M. Chem. Rev. 1947, 41, 401-419. doi:10.1021/cr60129a015

19. Lewis, G. N.; Calvin, M. J. Am. Chem. Soc. 1945, 67, 1232-1233. doi:10.1021/ja01223a513

20. Lewis, G. N.; Kasha, M. J. Am. Chem. Soc. 1944, 66, 2100-2116. doi:10.1021/ja01240a030

21. Lewis, G. N.; Lipkin, D.; Magel, T. T. J. Am. Chem. Soc. 1941, 63, 3005-3018. doi:10.1021/ja01856a043

22. S. K. Lower and M. A. El-Sayed; Lower, S. K.; El-Sayed, M. A. Chem. Rev. 1966, 66, 199-241. doi:10.1021/cr60240a004

23. El-Sayed, M. A. J. Chem. Phys. 1963, 38, 2834-2838. doi:10.1063/1.1733610

24. Hirata, S. Adv. Optical Mater. 2017, 5, 1700116. doi:10.1002/adom.201700116

25. Menning, S.; Kramer, M.; Coombs, B. A.; Rominger, F.; Beeby, A.; Dreuw, A.; Bunz, U. H. F. J. Am. Chem. Soc. 2013, 135, 2160-2163. doi:10.1021/ja400416r

26. Yuan, W. Z.; Shen, X. Y.; Zhao, H.; Lam, J. W. Y.; Tang, L.; Lu, P.; Wang, C.; Liu, Y.; Wang, Z.; Zheng, Q.; Sun, J. Z.; Ma, Y.; Tang, B. Z. J. Phys. Chem. C 2010, 114, 6090-6099. doi:10.1021/jp909388y

27. Zhang, G.; Chen, J.; Payne, S. J.; Kooi, S. E.; Demas, J. N.; Fraser, C. L. J. Am. Chem. Soc. 2007, 129, 8942-8943. doi:10.1021/ja0720255

28. Xu, J.; Takai, A.; Kobayashi, Y.; Takeuchi, M. Chem. Commun. 2013, 49, 8447-8449. doi:10.1039/c3ec44809f

29. Schulze, M.; Steffen, A.; Würthner, F. Angew. Chem. Int. Ed. 2015, 54, 1570-1573. doi:10.1002/anie.201410437

30. Hirata, S.; Totani, K.; Yamashita, T.; Adachi, C.; Vacha, M. Nat. Mater. 2014, 13, 938-946. doi:10.1038/nmat4081

31. Pan, Z.; Lu, Y.-Y.; Liu, F. Nat. Mater. 2011, 11, 58-63. doi:10.1038/nmat3173

32. Tong, B.; Mei, Q.; Wang, S.; Fang, Y.; Meng, Y.; Wang, B. J. Mater. Chem. 2008, 18, 1636-1639. doi:10.1039/b800977e 
33. Liu, Z. W.; Guan, M.; Bian, Z. Q.; Nie, D. B.; Gong, Z. L.; Li, Z. B.; Huang, C. H. Adv. Funct. Mater. 2006, 16, 1441-1448. doi:10.1002/adfm.200600099

34. Zhao, J.; Chen, K.; Hou, Y.; Che, Y.; Liu, L.; Jia, D. Org. Biomol. Chem. 2018, 16, 3692-3701. doi:10.1039/c8ob00421h

35. Forni, A.; Lucenti, E.; Botta, C.; Cariati, E. J. Mater. Chem. C 2018, 6, 4603-4626. doi:10.1039/C8TC01007B

36. Baroncini, M.; Bergamini, G.; Ceroni, P. Chem. Commun. 2017, 53, 2081-2093. doi:10.1039/c6ec09288h

37. Mukherjee, S.; Thilagar, P. Chem. Commun. 2015, 51, 10988-11003. doi:10.1039/c5cc03114a

38. Shi, H.; Song, L.; Ma, H.; Sun, C.; Huang, K.; Lv, A.; Ye, W.; Wang, H.; Cai, S.; Yao, W.; Zhang, Y.; Zheng, R.; An, Z.; Huang, W. J. Phys. Chem. Lett. 2019, 10, 595-600. doi:10.1021/acs.jpclett.8b03712

39. Li, J.-A.; Zhou, J.; Mao, Z.; Xie, Z.; Yang, Z.; Xu, B.; Liu, C.; Chen, X.; Ren, D.; Pan, H.; Shi, G.; Zhang, Y.; Chi, Z. Angew. Chem. Int. Ed. 2018, 57, 6449-6453. doi:10.1002/anie.201800762

40. Xiong, Y.; Zhao, Z.; Zhao, W.; Ma, H.; Peng, Q.; He, Z.; Zhang, X.; Chen, Y.; He, X.; Lam, J. W. Y.; Tang, B. Z. Angew. Chem. Int. Ed. 2018, 57, 7997-8001. doi:10.1002/anie.201800834

41. Gu, L.; Shi, H.; Miao, C.; Wu, Q.; Cheng, Z.; Cai, S.; Gu, M.; Ma, C.; Yao, W.; Gao, Y.; An, Z.; Huang, W. J. Mater. Chem. C 2018, 6, 226-233. doi:10.1039/C7TC04452F

42. Cai, S.; Shi, H.; Zhang, Z.; Wang, X.; Ma, H.; Gan, N.; Wu, Q.; Cheng, Z.; Ling, K.; Gu, M.; Ma, C.; Gu, L.; An, Z.; Huang, W. Angew. Chem. Int. Ed. 2018, 57, 4005-4009. doi:10.1002/anie.201800697

43. Cai, S.; Shi, H.; Tian, D.; Ma, H.; Cheng, Z.; Wu, Q.; Gu, M.; Huang, L.; An, Z.; Peng, Q.; Huang, W. Adv. Funct. Mater. 2018, 28, 1705045. doi:10.1002/adfm.201705045

44. Cai, S.; Shi, H.; Li, J.; Gu, L.; Ni, Y.; Cheng, Z.; Wang, S.; Xiong, W.-W.; Li, L.; An, Z.; Huang, W. Adv. Mater. 2017, 29, 1701244. doi:10.1002/adma.201701244

45. Yang, Z.; Mao, Z.; Zhang, X.; Ou, D.; Mu, Y.; Zhang, Y.; Zhao, C.; Liu, S.; Chi, Z.; Xu, J.; Wu, Y.C.; Lu, P.-Y.; Lien, A.; Bryce, M. R. Angew. Chem. Int. Ed. 2016, 55, 2181-2185. doi:10.1002/anie.201509224 
46. Gong, Y.; Chen, G.; Peng, Q.; Yuan, W. Z.; Xie, Y.; Li, S.; Zhang, Y.; Tang, B. Z. Adv. Mater. 2015, 27, 6195-6201. doi:10.1002/adma.201502442

47. An, Z.; Zheng, C.; Tao, Y.; Chen, R.; Shi, H.; Chen, T.; Wang, Z.; Li, H.; Deng, R.; Liu, X.; Huang, W. Nat. Mater. 2015, 14, 685-690. doi:10.1038/nmat4259

48. Yu, Z.; Wu, Y.; Peng, Q.; Sun, C.; Chen, J.; Yao, J.; Fu, H. Chem. Eur. J. 2016, 22, 4717-4722. doi:10.1002/chem.201600300

49. Chen, X.; Xu, C.; Wang, T.; Zhou, C.; Du, J.; Wang, Z.; Xu, H.; Xie, T.; Bi, G.; Jiang, J.; Zhang, X.; Demas, J. N.; Trindle, C. O.; Luo, Y.; Zhang, G. Angew. Chem. Int. Ed. 2016, 55, 9872-9876. doi:10.1002/anie.201601252

50. Lin, Z.; Kabe, R.; Nishimura, N.; Jinnai, K.; Adachi, C. Adv. Mater. 2018, 30, 1803713. doi:10.1002/adma.201803713

51. Li, D.; Lu, F.; Wang, J.; Hu, W.; Cao, X.-M.; Ma, X.; Tian, H. J. Am. Chem. Soc. 2018, 140, 19161923. doi:10.1021/jacs.7b12800

52. Kabe, R.; Adachi, C. Nature 2017, 550, 384-387. doi:10.1038/nature24010

53. Gan, N.; Wang, X.; Ma, H.; Lv, A.; Wang, H.; Wang, Q.; Gu, M.; Cai, S.; Zhang, Y.; Fu, L.; Zhang, M.; Dong, C.; Yao, W.; Shi, H.; An, Z.; Huang, W. Angew. Chem. Int. Ed. 2019, 58, 14140-14145. doi:10.1002/anie.201907572

54. Yang, J.; Ren, Z.; Chen, B.; Fang, M.; Zhao, Z.; Tang, B. Z.; Peng, Q.; Li, Z. J. Mater. Chem. C 2017, 5, 9242-9246. doi:10.1039/C7TC03656F

55. Shimizu, M.; Shigitani, R.; Nakatani, M.; Kuwabara, K.; Miyake, Y.; Tajima, K.; Sakai, H.; Hasobe, T. J. Phys. Chem. C 2016, 120, 11631-11639. doi:10.1021/acs.jpcc.6b03276

56. Xie, Y.; Ge, Y.; Peng, Q.; Li, C.; Li, Q.; Li, Z. Adv. Mater. 2017, 29, 1606829. doi:10.1002/adma.201606829

57. Bergamini, G.; Fermi, A.; Botta, C.; Giovanella, U.; Di Motta, S.; Negri, F.; Peresutti, R.; Gingras, M.; Ceroni, P. J. Mater. Chem. C 2013, 1, 2717-2724. doi:10.1039/c3tc00878a

58. Bolton, O.; Lee, K.; Kim, H.-J.; Lin, K. Y.; Kim, J. Nat. Chem. 2011, 3, 205-210. doi:10.1038/nchem.984 
59. Yu, Y.; Kwon, M. S.; Jung, J.; Zeng, Y.; Kim, M.; Chung, K.; Gierschner, J.; Youk, J. H.; Borisov, S. M.; Kim, J. Angew. Chem. Int. Ed. 2017, 56, 16207-16211. doi:10.1002/anie.201708606

60. Kwon, M. S.; Jordahl, J. H.; Phillips, A. W.; Chung, K.; Lee, S.; Gierschner, J.; Lahann, J.; Kim, J. Chem. Sci. 2016, 7, 2359-2363. doi:10.1039/C5SC03986J

61. Kwon, M. S.; Yu, Y.; Coburn, C.; Phillips, A. W.; Chung, K.; Shanker, A.; Jung, J.; Kim, G.; Pipe, K.; Forrest, S. R.; Youk, J. H.; Gierschner, J.; Kim, J. Nat. Commun. 2015, 6, 8947. doi:10.1038/ncomms9947

62. Lee, D.; Jung, J.; Bilby, D.; Kwon, M. S.; Yun, J.; Kim, J. ACS Appl. Mater. Interfaces 2015, 7, 2993-2997. doi:10.1021/am5087165

63. Bolton, O.; Lee, D.; Jung, J.; Kim, J. Chem. Mater. 2014, 26, 6644-6649. doi:10.1021/cm503678r

64. Goudappagouda; Manthanath, A.; Wakchaure, V. C.; Ranjeesh, K. C.; Das, T.; Vanka, K.; Nakanishi, T.; Babu, S. S. Angew. Chem. Int. Ed. 2019, 58, 2284-2288. doi:10.1002/anie.201811834

65. Kuila, S.; Rao, K. V.; Garain, S.; Samanta, P. K.; Das, S.; Pati, S. K.; Eswaramoorthy, M.; George, S. J. Angew. Chem. Int. Ed. 2018, 57, 17115-17119. doi:10.1002/anie.201810823

66. Yu, Z.; Wu, Y.; Xiao, L.; Chen, J.; Liao, Q.; Yao, J.; Fu, H. J. Am. Chem. Soc. 2017, 139, 63766381. doi:10.1021/jacs.7b01574

67. Huang, C.-H.; Wu, P.-J.; Chung, K.-Y.; Chen, Y.-A.; Li, E. Y.; Chou, P.-T. Phys. Chem. Chem. Phys. 2017, 19, 8896-8901. doi:10.1039/c7cp00074j

68. Ventura, B.; Bertocco, A.; Braga, D.; Catalano, L.; d'Agostino, S.; Grepioni, F.; Taddei, P. J. Phys. Chem. C 2014, 118, 18646-18658. doi:10.1021/jp5049309

69. Koch, M.; Perumal, K.; Blacque, O.; Garg, J. A.; Saiganesh, R.; Kabilan, S.; Balasubramanian, K. K.; Venkatesan, K. Angew. Chem. Int. Ed. 2014, 53, 6378-6382. doi:10.1002/anie.201402199

70. Göbel, D.; Clamor, N.; Nachtsheim, B. J. Org. Biomol. Chem. 2018, 16, 4071-4075. doi:10.1039/c8ob01072b

71. Göbel, D.; Clamor, N.; Lork, E.; Nachtsheim, B. J. Org. Lett. 2019, 21, 5373-5377. doi:10.1021/acs.orglett.9b01350 
72. Sarkar, S.; Hendrickson, H. P.; Lee, D.; DeVine, F.; Jung, J.; Geva, E.; Kim, J.; Dunietz, B. D. J. Phys. Chem. C 2017, 121, 3771-3777. doi:10.1021/acs.jpcc.6b12027

73. Xie, S.; Proietti, G.; Ramström, O.; Yan, M. J. Org. Chem. 2019, 84, 14520-14528. doi:10.1021/acs.joc.9b02050

74. Lord, S. J.; Lee, H.-L. D.; Samuel, R.; Weber, R.; Liu, N.; Conley, N. R.; Thompson, M. A.; Twieg, R. J.; Moerner, W. E. J. Phys. Chem. B 2010, 114, 14157-14167. doi:10.1021/jp907080r 


\title{
Clickable Azide-functionalized Bromo-Aryl-Aldehydes - Synthesis and Photophysical Characterization
}

\author{
Supporting Information \\ Dominik Göbel ${ }^{1}$, Marius Friedrich ${ }^{1,2}$, Enno Lork ${ }^{3}$, Boris J. Nachtsheim ${ }^{* 1}$ \\ ${ }^{1}$ Institute for Organic and Analytical Chemistry, University of Bremen, \\ Leobener Straße NW2, D-28359 Bremen, Germany, \\ ${ }^{2}$ Department of Organic Chemistry, Technical University Kaiserslautern, \\ Erwin-Schrödinger-Straße Geb.54, D-67663 Kaiserslautern, Germany, \\ ${ }^{3}$ Institute for Inorganic and Crystallographic Chemistry, University of Bremen, \\ Leobener Straße NW2, D-28359 Bremen, Germany
}

*Corresponding author: nachtsheim@uni-bremen.de 


\section{Table of Content}

1 General Information ........................................................................................................................... 1

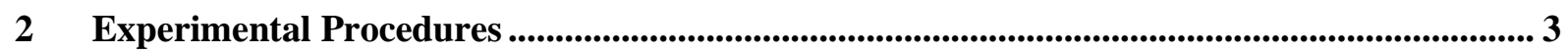

2.1 Preparation of Metalation Agents .................................................................................................. 3

2.1.1 Preparation of the Reagent $i \mathrm{PrMgCl} \cdot \mathrm{LiCl}$............................................................................. 3

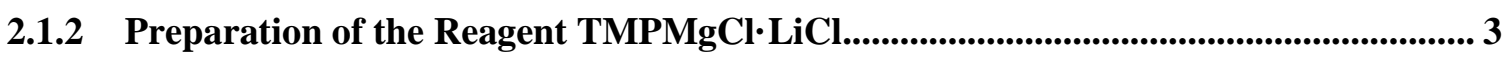

2.2 Preparation of 2-(Azidomethyl)-4-bromobenzaldehyde (3) .............................................. 4

2.2.1 2-(4-Bromophenyl)-4,4-dimethyl-4,5-dihydrooxazole (6)................................................... 4

2.2.2 5-Bromo-2-(4,4-dimethyl-4,5-dihydrooxazol-2-yl)benzaldehyde (7) ............................ 5

2.2.3 (5-Bromo-2-(4,4-dimethyl-4,5-dihydrooxazol-2-yl)phenyl)methanol (8) ....................... 6

2.2.4 2-(2-(Azidomethyl)-4-bromophenyl)-4,4-dimethyl-4,5-dihydrooxazole (9) .................. 6

2.2.5 2-(Azidomethyl)-4-bromobenzaldehyde (3) .................................................................... 7

2.3 Preparation of 4-(Azidomethyl)-2-bromobenzaldehyde (4) ...................................................... 8

2.3.1 4,4-Dimethyl-2-(p-tolyl)-4,5-dihydrooxazole (10).......................................................... 8

2.3.2 2-(4-(Bromomethyl)phenyl)-4,4-dimethyl-4,5-dihydrooxazole (S1) ............................. 9

2.3.3 2-(4-(Azidomethyl)phenyl)-4,4-dimethyl-4,5-dihydrooxazole (S2).............................. 10

2.3.4 2-(2-Bromo-4-methylphenyl)-4,4-dimethyl-4,5-dihydrooxazole (11) .......................... 10

2.3.5 2-(2-Bromo-4-(bromomethyl)phenyl)-4,4-dimethyl-4,5-dihydrooxazole (12) ............ 11

2.3.6 2-(4-(Azidomethyl)-2-bromophenyl)-4,4-dimethyl-4,5-dihydrooxazole (13) .............. 12

2.3.7 4-(Azidomethyl)-2-bromobenzaldehyde (4) .................................................................. 12

2.4 Preparation of Highly Functionalized Fluorenes (5), (23), (25) \& (28) .............................. 14

2.4.1 2,7-Dibromo-9H-fluorene (14) ................................................................................................... 15

2.4.2 2,7-Dibromo-9,9-dimethyl-9H-fluorene (15).................................................................. 15

2.4.3 7-Bromo-9,9-dimethyl-9H-fluorene-2-carbaldehyde (16) ............................................... 16

2.4.4 2-(7-Bromo-9,9-dimethyl-9H-fluoren-2-yl)-4,4-dimethyl-4,5-dihydrooxazole (17)... 17

2.4.5 7-Bromo-2-(4,4-dimethyl-4,5-dihydrooxazol-2-yl)-9,9-dimethyl-9H-fluorene-3-

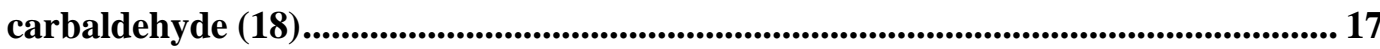

2.4.6 (7-Bromo-2-(4,4-dimethyl-4,5-dihydrooxazol-2-yl)-9,9-dimethyl-9H-fluoren-3-

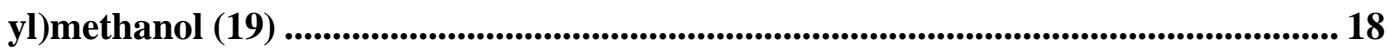

2.4.7 7-Bromo-9,9-dimethyl-3,9-dihydro-1H-fluoreno[2,3-c]furan-1-one (20)..................... 19

2.4.8 2-(3-(Azidomethyl)-7-bromo-9,9-dimethyl-9H-fluoren-2-yl)-4,4-dimethyl-4,5dihydrooxazole (21)...................................................................................................... 20

2.4.9 3-(Azidomethyl)-7-bromo-9,9-dimethyl-9H-fluorene-2-carbaldehyde (5) .................. 21

2.4.10 2-(7-Bromo-3-(methoxymethyl)-9,9-dimethyl-9H-fluoren-2-yl)-4,4-dimethyl-4,5-

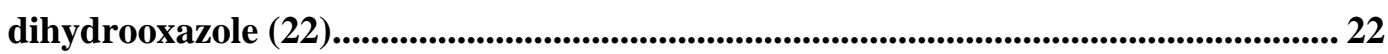

2.4.11 7-Bromo-3-(methoxymethyl)-9,9-dimethyl-9H-fluorene-2-carbaldehyde (23).......... 22 
2.4.12 9-Bromo-3,3,11,11-tetramethyl-2,3,5,11-tetrahydroindeno[1,2-f]oxazolo[2,3a]isoindol-4-ium bromide (25)

2.4.13 9-Bromo-3,3,4,11,11-pentamethyl-2,3,4,5,11,12b-hexahydroindeno[1,2-f]oxazolo[2,3a]isoindol-4-ium trifluoromethanesulfonate (28) .

2.4.14 Attempted Preparation of (7-bromo-2-(4,4-dimethyl-4,5-dihydrooxazol-2-yl)-9,9dimethyl-9H-fluoren-3-yl)methyl methanesulfonate (S3)

2.5 Preparation of Sterically Hindered Alkynes. 27

2.5.1 Preparation of $(3 r, 5 r, 7 r)$-1-ethynyladamantane $(31)$......................................................... 27

2.5.2 Preparation of 1,3-di-tert-butyl-5-ethynylbenzene (32) ..................................................... 28

2.5.2.1 3,5-Di-tert-butylbenzaldehyde (S4) .................................................................................... 28

2.5.2.2 1,3-Di-tert-butyl-5-(2,2-dibromovinyl)benzene (S5) ................................................ 29

2.5.3 1,3-Di-tert-butyl-5-ethynylbenzene (32) ..................................................................................... 30

2.6 Copper-Catalyzed Azide-Alkyne Cycloadditions (CuAAC) .......................................... 31

2.6.1 General Procedure for the CuAACs (GP1) ............................................................. 31

2.6.2 4-Bromo-2-((4-octyl-1H-1,2,3-triazol-1-yl)methyl)benzaldehyde (33) .......................... 31

2.6.3 2-((4-((1s,3s)-Adamantan-1-yl)-1H-1,2,3-triazol-1-yl)methyl)-4-bromobenzaldehyde (34). 32

2.6.4 4-Bromo-2-((4-phenyl-1H-1,2,3-triazol-1-yl)methyl)benzaldehyde (35) 32

2.6.5 4-Bromo-2-((4-(3,5-di-tert-butylphenyl)-1H-1,2,3-triazol-1-yl)methyl)benzaldehyde (36)

2.6.6 2-Bromo-4-((4-octyl-1H-1,2,3-triazol-1-yl)methyl)benzaldehyde (37) 33

2.6.7 4-((4-((1s,3s)-Adamantan-1-yl)-1H-1,2,3-triazol-1-yl)methyl)-2-bromobenzaldehyde (38)

2.6.8 2-Bromo-4-((4-phenyl-1H-1,2,3-triazol-1-yl)methyl)benzaldehyde (39) 35

2.6.9 2-Bromo-4-((4-(3,5-di-tert-butylphenyl)-1H-1,2,3-triazol-1-yl)methyl)benzaldehyde (40) 35

2.6.10 7-Bromo-9,9-dimethyl-3-((4-octyl-1H-1,2,3-triazol-1-yl)methyl)-9H-fluorene-2carbaldehyde (41).

2.6.11 3-((4-((3r,5r,7r)-Adamantan-1-yl)-1H-1,2,3-triazol-1-yl)methyl)-7-bromo-9,9dimethyl-9H-fluorene-2-carbaldehyde (42)

2.6.12 7-Bromo-9,9-dimethyl-3-((4-phenyl-1H-1,2,3-triazol-1-yl)methyl)-9H-fluorene-2carbaldehyde (43)

2.6.13 7-Bromo-3-((4-(3,5-di-tert-butylphenyl)-1H-1,2,3-triazol-1-yl)methyl)-9,9-dimethyl$9 H$-fluorene-2-carbaldehyde (44) 38

2.7 Methylation Reaction of Adamantyl substituted triazole 42 . 39

2.7.1 4-((3r,5r,7r)-Adamantan-1-yl)-1-((7-bromo-2-formyl-9,9-dimethyl-9H-fluoren-3yl)methyl)-3-methyl-1H-1,2,3-triazol-3-ium tetrafluoroborate (45) 


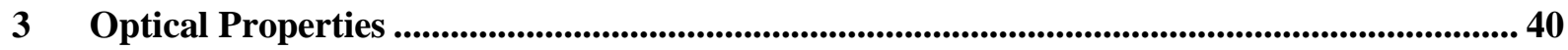

3.1 2-(Azidomethyl)-4-bromobenzaldehyde (3) \& Derivatives ................................................. 40

3.2 4-(Azidomethyl)-2-bromobenzaldehyde (4) \& Derivatives .................................................. 43

3.3 3-(Azidomethyl)-7-bromo-9,9-dimethyl-9H-fluorene-2-carbaldehyde (5) \& Derivatives 45

4 Crystal Structure of 3-(Azidomethyl)-7-bromo-9,9-dimethyl-9H-fluorene-2-carbaldehyde (5) 62

4.1 Crystal Data for (5) ................................................................................................................. 62

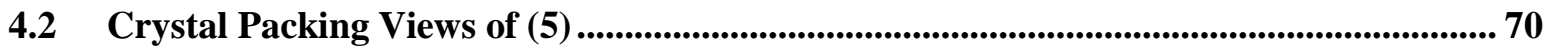

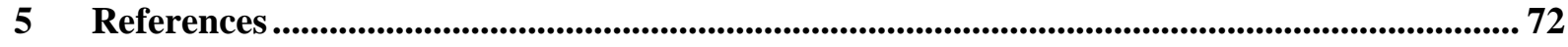

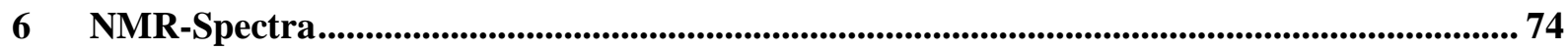




\section{General Information}

Unless otherwise noted, all reactions were carried out under a nitrogen atmosphere using standard Schlenk techniques. All chemicals were purchased from commercial suppliers and either used as received or purified according to Purification of Common Laboratory Chemicals.[1] Anhydrous tetrahydrofuran (THF) and dichloromethane $\left(\mathrm{CH}_{2} \mathrm{Cl}_{2}\right)$ were obtained from an inert PS-MD-6 solvent purification system. All other solvents were dried using standard methods.[1] Yields refer to isolated yields of compounds estimated to be $>95 \%$ pure as determined by ${ }^{1} \mathrm{H}-\mathrm{NMR}$ spectroscopy.

Thin layer chromatography was performed on fluorescence indicator marked precoated silica gel 60 plates (Macherey-Nagel, ALUGRAM Xtra SIL G/UV $\mathrm{UV}_{254}$ ) and visualized by UV light $(254 \mathrm{~nm} / 366 \mathrm{~nm})$ or staining solution. $\mathrm{KMnO}_{4}$ staining solution was prepared using the following recipe: $\mathrm{KMnO}_{4}(1.5 \mathrm{~g}), \mathrm{K}_{2} \mathrm{CO}_{3}(10 \mathrm{~g})$ and aqueous $\mathrm{NaOH}(1.25 \mathrm{~mL}, 10 \%)$ in water $(200 \mathrm{~mL})$. Flash column chromatography was performed on silica gel $(0.040-0.063 \mathrm{~mm})$ with the solvents given in the procedures. Abbreviations for solvents used: $\mathrm{CH}=$ cyclohexane, $\mathrm{EE}=$ ethyl acetate. Retention factors were determined at chamber saturation at $25{ }^{\circ} \mathrm{C}$. Developments were carried out between $3.0-3.5 \mathrm{~cm}$. If not otherwise stated, crude products were adsorbed on silica gel (up to twice the amount of crude product mass) prior to purification by flash column chromatography.

NMR spectra were recorded on a Bruker Avance 360WB spectrometer, a Bruker Avance Neo $600 \mathrm{MHz}$ spectrometer with BBO probe head and a Bruker Avance Neo $600 \mathrm{MHz}$ spectrometer with TXI probe head at $23{ }^{\circ} \mathrm{C}$. Chemical shifts for ${ }^{1} \mathrm{H}-\mathrm{NMR}$ spectra are reported as $\delta$ (parts per million) relative to the residual proton signal of $\mathrm{CDCl}_{3}$ at $7.26 \mathrm{ppm}(\mathrm{s}), \mathrm{MeOD}-d_{4}$ at $3.31 \mathrm{ppm}(\mathrm{p})$. Chemical shifts for ${ }^{13} \mathrm{C}-\mathrm{NMR}$ spectra are reported as $\delta$ (parts per million) relative to the signal of $\mathrm{CDCl}_{3}$ at $77.0 \mathrm{ppm}(\mathrm{t}), \mathrm{MeOD}-d_{4}$ at $49.0 \mathrm{ppm}$ (sept). Chemical shifts for ${ }^{19} \mathrm{~F}-\mathrm{NMR}$ spectra are reported as $\delta$ (parts per million) relative to the signal of $\mathrm{Si}\left(\mathrm{CH}_{3}\right)_{4}$ at $0.0 \mathrm{ppm}$. The following abbreviations are used to describe splitting patterns: br. = broad, $\mathrm{s}=$ singlet, $\mathrm{d}=$ doublet, $\mathrm{dd}=$ doublet of doublets, $\mathrm{ddd}=$ doublet of doublet of doublets, $\mathrm{t}=$ triplet, $\mathrm{p}=$ pentet, $\mathrm{m}=$ multiplet. Coupling constants $J$ are given in Hertz.

ESI and APCI mass spectra were recorded on an Advion Expression $\mathrm{CMS}^{\mathrm{L}}$ via ASAP probe or direct inlet. EI mass spectra were obtained from an Agilent 7890B GC System with an Agilent 5977A MSD mass spectrometer. As column a HP-5M5 (5\%-phenyl)-methylpolysiloxane was used. High resolution (HR) EI mass spectra were recorded on a double focusing mass spectrometer ThermoQuest MAT 95 XL from Finnigan MAT. HR-ESI and HR-APCI mass spectra were recorded on a Bruker Impact II. All Signals are reported with the quotient from mass to charge $\mathrm{m} / \mathrm{z}$. 
IR spectra were recorded on a Nicolet Thermo iS10 scientific spectrometer with a diamond ATR unit. The absorption bands are reported in $\mathrm{cm}^{-1}$ with indicated relative intensities: $\mathrm{s}$ (strong, $0-33 \% \mathrm{~T}$ ); $\mathrm{m}$ (medium, $34-66 \%$ T), w (weak, $67-100 \%$ T), and br (broad).

Melting points of solids, compounds that solidified after chromatography, were measured on a Büchi M-5600 Melting Point apparatus and are uncorrected. The measurements were performed with a heating rate of $5{ }^{\circ} \mathrm{C} / \mathrm{min}$ and the melting points are reported in ${ }^{\circ} \mathrm{C}$.

Low temperature reactions $\left(-78^{\circ} \mathrm{C}\right)$ were cooled using a Julabo FT902 cryostat. If not otherwise noted, solvents were removed on a Büchi Rotavapor R-300 with $40{ }^{\circ} \mathrm{C}$ water bath temperature.

Absorption measurements were performed either using an Agilent Cary 5000 UV-Vis-NIR spectrophotometer or a Shimadzu UV-2700 UV-Vis spectrophotometer. Emission measurements were performed using an Edinburgh Instruments FLS 1000 photoluminescence spectrometer. Absolute quantum yields were measured using an Edinburgh integrating sphere. TCSPC measurements were performed using a fast response MCP-PMT detector on the FLS 1000 and a $376 \mathrm{~nm}$ Edinburgh EPL Laser as excitation source with $10-20 \mathrm{MHz}$ repetition rate and $80 \mathrm{ps}$ pulse width. All measurements were performed at $23{ }^{\circ} \mathrm{C}$ room temperature in Quartz Cuvettes with $10 \mathrm{~mm}$ path length by Hellma Analytics.

1

Single crystals were grown as described in the procedure below. Intensity data of suitable crystals were collected on a Bruker Venture D8 diffractometer at $100 \mathrm{~K}$ with Mo-K $\alpha(0.71073 \AA$ A) radiation. All structures were solved by direct methods and refined based on $\mathrm{F}^{2}$ by use of the SHELX program package as implemented in Olex2.[2, 3] All non-hydrogen atoms were refined using anisotropic displacement parameters. Hydrogen atoms attached to carbon atoms were included in geometrically calculated positions using a rigid model. Crystal and refinement data are collected in Tables S4-22. Figures were created using Olex2. Crystallographic data for the structural analyses have been deposited with the Cambridge Crystallographic Data Centre. Copies of this information may be obtained free of charge from The Director, CCDC, 12 Union Road, Cambridge CB2 1EZ, UK (Fax: +44-1223-336033; e-mail: deposit@ccdc.cam.ac.uk or http://www.ccdc.cam.ac.uk). 


\section{Experimental Procedures}

\subsection{Preparation of Metalation Agents}

\subsubsection{Preparation of the Reagent $i \mathrm{PrMgCl} \cdot \mathrm{LiCl}$}

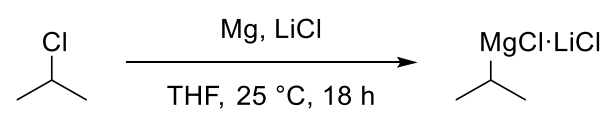

A slightly modified literature procedure was used.[4] $\mathrm{LiCl}(4.24 \mathrm{~g}, 100 \mathrm{mmol}, 1.00 \mathrm{eq})$ was placed in a heat gun-dried and nitrogen-flushed Schlenk flask and heated in vacuo at $140{ }^{\circ} \mathrm{C}$ by heat gun for five hours. Magnesium turnings $(2.67 \mathrm{~g}, 110 \mathrm{mmol}, 1.10 \mathrm{eq})$ were placed in another heat gun-dried and nitrogen-flushed Schlenk flask and the dried $\mathrm{LiCl}$ and anhydrous THF $(50 \mathrm{~mL})$ were added. 2-Chloropropane $(9.14 \mathrm{~mL}, 100 \mathrm{mmol}, 1.00 \mathrm{eq})$ in anhydrous THF $(50 \mathrm{~mL})$ was slowly added at $25^{\circ} \mathrm{C}$ through a dropping funnel. After approximately $1 / 5$ of addition the mixture was slightly warmed with a heat gun until the reaction started (within ten minutes). When the reaction started the remaining solution was added dropwise and stirring was continued for 18 hours. After complete addition the temperature of the mixture rose until it started to boil. To remove excess of magnesium the grey solution was cannulated to another heat gun-dried and nitrogen-flushed Schlenk flask. The Grignard reagent was titrated[5] prior to use against $\mathrm{I}_{2}(0.50-0.60 \mathrm{mmol})$ in anhydrous THF $(2 \mathrm{~mL})$ at $0{ }^{\circ} \mathrm{C}$ which resulted in a conversion of $92-96 \%$. Color change from dark violet to pale brown indicated the end of the titration.

\subsubsection{Preparation of the Reagent TMPMgCl$\cdot \mathbf{L i C l}$}

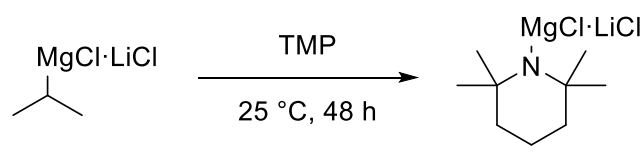

A slightly modified literature procedure was used.[6] A heat gun-dried and nitrogen-flushed Schlenk flask was charged with freshly titrated $i \mathrm{PrMgCl} \cdot \mathrm{LiCl}(75.0 \mathrm{~mL}, 90.0 \mathrm{mmol}, 1.00 \mathrm{eq}, 1.20 \mathrm{M})$. Freshly distilled TMP (16.0 mL, $94.5 \mathrm{mmol}, 1.05 \mathrm{eq})$ was added through a rubber septum to the vigorously stirred Grignard solution via syringe pump $(0.5 \mathrm{~mL} / \mathrm{min})$ at $25^{\circ} \mathrm{C}$. The reaction mixture was stirred at $25{ }^{\circ} \mathrm{C}$ for 48 hours, while the solution turned dark green. The base was titrated[7] prior to use against benzoic acid (122 mg, $1.00 \mathrm{mmol}$ ) using (4-phenylazo)diphenylamine ( $3 \mathrm{mg}$ ) as indicator in anhydrous THF $(2.00 \mathrm{~mL})$ at $0{ }^{\circ} \mathrm{C}$ which resulted in a conversion of $96-99 \%$. Color change from orange to dark violet indicated the end of the titration. 


\subsection{Preparation of 2-(Azidomethyl)-4-bromobenzaldehyde (3)}<smiles>CC(C)C=O</smiles><smiles>CC1(C)COC(c2ccc(Br)cc2)=N1</smiles>

6

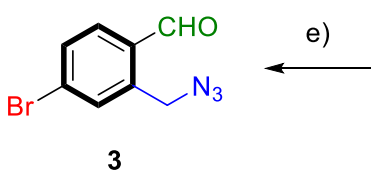

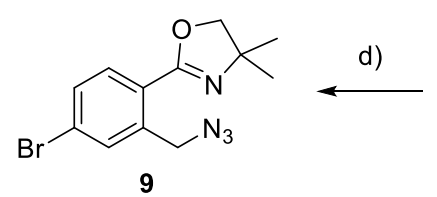

Reaction conditions: a) 1) 2-amino-2-methylpropan-1-ol, $4 \AA$ MS, $\mathrm{CH}_{2} \mathrm{Cl}_{2}, 25^{\circ} \mathrm{C}, 18 \mathrm{~h}$; 2) NBS, $\mathrm{CH}_{2} \mathrm{Cl}_{2}, 25^{\circ} \mathrm{C}, 5 \mathrm{~h}, 91 \%$; b) 1) TMPMgCl$\cdot \mathrm{LiCl}, \mathrm{THF}, 25^{\circ} \mathrm{C}, 4 \mathrm{~h}$; 2 ) DMF, THF, $0{ }^{\circ} \mathrm{C}$ to $25^{\circ} \mathrm{C}, 1.5 \mathrm{~h}$; c) $\mathrm{NaBH}_{4}$, THF:MeOH 1:1, $0{ }^{\circ} \mathrm{C}, 1 \mathrm{~h}, 81 \%$ (2 steps); d) DPPA, DBU, PhMe, $25{ }^{\circ} \mathrm{C}, 18$ h, $98 \%$; e) 1) MeOTf, $\mathrm{CH}_{2} \mathrm{Cl}_{2}, 25^{\circ} \mathrm{C}, 2.5 \mathrm{~h}$; 2) $\mathrm{NaBH}_{4}$, THF:MeOH 4:1 v:v, $0{ }^{\circ} \mathrm{C}$, $2.5 \mathrm{~h} ; 3$ ) oxalic acid, THF: $\mathrm{H}_{2} \mathrm{O} 4: 1$ v:v, $25^{\circ} \mathrm{C}, 20$ h, $78 \%$. Overall yield: $56 \%$ (5 steps).

\subsubsection{2-(4-Bromophenyl)-4,4-dimethyl-4,5-dihydrooxazole (6)}<smiles>O=Cc1ccc(Br)cc1</smiles>

1) 2-amino-2-methylpropan-1-ol, $4 \AA \mathrm{MS}, \mathrm{CH}_{2} \mathrm{Cl}_{2}, 25^{\circ} \mathrm{C}, 18 \mathrm{~h}$

2) NBS, $\mathrm{CH}_{2} \mathrm{Cl}_{2}, 25^{\circ} \mathrm{C}, 5 \mathrm{~h}$ $91 \%$<smiles>CC1(C)COC(c2ccc(Br)cc2)=N1</smiles>

A modified literature procedure was used.[8] 4-Bromobenzaldehyde (5.55 g, $30.0 \mathrm{mmol}, 1.00 \mathrm{eq})$ and 2-amino-2-methylpropan-1-ol (5.35 g, $60.0 \mathrm{mmol}, 2.00 \mathrm{eq})$ were dissolved in $\mathrm{CH}_{2} \mathrm{Cl}_{2}(100 \mathrm{~mL}, 0.3 \mathrm{M})$ and $4 \AA$ A MS (20.0 g) was added. After 18 hours of slowly stirring at $25^{\circ} \mathrm{C}$ NBS $(10.7 \mathrm{~g}, 60.0 \mathrm{mmol}$, $2.00 \mathrm{eq}$ ) was added in one portion and stirring was continued for another five hours at $25^{\circ} \mathrm{C}$. All solids were filtered off and the organic phase was washed with saturated $\mathrm{NaHCO}_{3}$ solution $(100 \mathrm{~mL})$. The combined aqueous phases were extracted with $\mathrm{CH}_{2} \mathrm{Cl}_{2}(3 \times 50 \mathrm{~mL})$. All organic phases were washed with saturated $\mathrm{NaS}_{2} \mathrm{O}_{3}$ solution $(100 \mathrm{~mL})$ and the aqueous phase was extracted with $\mathrm{CH}_{2} \mathrm{Cl}_{2}(50 \mathrm{~mL})$. The combined organic phases were dried over anhydrous $\mathrm{MgSO}_{4}$, filtered and the solvent was removed under reduced pressure. Purification by flash column chromatography $\left(\mathrm{SiO}_{2}, \mathrm{CH}: \mathrm{EE}\right.$ 10:1 v:v) afforded $\mathbf{6}(6.97 \mathrm{~g}, 27.4 \mathrm{mmol}, 91 \%)$ as a colorless oil which solidified upon standing to form a pale brown solid.

$\mathbf{R}_{f}=0.26\left(\mathrm{SiO}_{2}\right.$, CH:EE 10:1 v:v). Mp.: $38-40{ }^{\circ} \mathrm{C} .{ }^{1} \mathbf{H}-\mathrm{NMR}\left(\mathbf{3 6 0} \mathbf{M H z}, \mathbf{C D C l}_{3}\right): \delta=7.83-7.74$

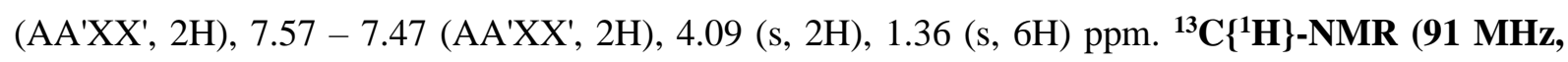
$\left.\mathbf{C D C l}_{3}\right): \delta=161.4,131.6(2 \mathrm{x}), 129.9$ (2x), 127.1, 125.9, 79.3, 67.9, 28.5 (2x) ppm. IR (ATR, neat): $\tilde{v}=2969(\mathrm{~m}), 2624(\mathrm{w}), 2897(\mathrm{w}), 1642(\mathrm{~s}), 1591(\mathrm{~m}), 1479(\mathrm{~m}), 1459(\mathrm{~m}), 1397(\mathrm{~m}), 1357$ (m), 1315 (m), 1296 (m), 1177 (m), 1070 (s), 1063 (s), 1009 (s), 964 (m), 923 (m), 835 (s), 824 (m), 726 (s), 712 
(m), $676(\mathrm{~s}) \mathrm{cm}^{-1}$. MS (APCI): $\mathrm{m} / \mathrm{z}=254.0\left[\mathrm{C}_{11} \mathrm{H}_{12}{ }^{79} \mathrm{BrNO}+\mathrm{H}\right]^{+}$. HR-MS (APCI): calculated for $\mathrm{C}_{11} \mathrm{H}_{13}{ }^{79} \mathrm{BrNO}^{+}[\mathrm{M}+\mathrm{H}]^{+}: \mathrm{m} / \mathrm{z}=254.01750$, found: 254.01748 (Dev.: $-0.02 \mathrm{mu} ;-0.10 \mathrm{ppm}$ ). The analytical data are in accordance with the literature.[9]

\subsubsection{5-Bromo-2-(4,4-dimethyl-4,5-dihydrooxazol-2-yl)benzaldehyde (7)}<smiles>CC1(C)COC(c2ccc(Br)cc2)=N1</smiles>

6

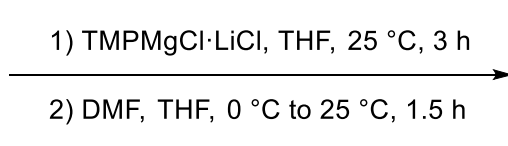

2) DMF, THF, $0{ }^{\circ} \mathrm{C}$ to $25^{\circ} \mathrm{C}, 1.5 \mathrm{~h}$

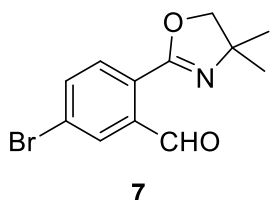

7

A modified literature procedure was used.[9] A heat gun-dried and nitrogen-flushed Schlenk flask, equipped with a magnetic stirring bar and a rubber septum was charged with 6 ( $3.30 \mathrm{~g}, 13.0 \mathrm{mmol}$, $1.00 \mathrm{eq}$ ) and evacuated for half an hour. The flask was flushed with nitrogen and anhydrous THF $(32.5 \mathrm{~mL}, 0.4 \mathrm{M})$ was added. Addition of TMPMgCl$\cdot \mathrm{LiCl}(26.0 \mathrm{~mL}, 26.0 \mathrm{mmol}, 2.00 \mathrm{eq}, 1.00 \mathrm{M}$ in THF) via syringe within three minutes at $25{ }^{\circ} \mathrm{C}$ was followed by stirring for three hours at the same conditions. DMF (4.03 mL, $52.0 \mathrm{mmol}, 4.00 \mathrm{eq}$ ) was diluted in anhydrous THF (10 mL) and added via syringe pump $(0.5 \mathrm{~mL} / \mathrm{min})$ to the dark red solution at $0{ }^{\circ} \mathrm{C}$. After complete addition the mixture was stirred for one and a half hours while it was allowed to warm to $25^{\circ} \mathrm{C}$. Full consumption of the starting material was followed by diluting with water $(50 \mathrm{~mL})$ and ethyl acetate $(50 \mathrm{~mL})$. The phases were separated and the aqueous layer was extracted with ethyl acetate $(4 \times 50 \mathrm{~mL})$. The combined organic phases were dried over anhydrous $\mathrm{NaSO}_{4}$, filtered and concentrated under reduced pressure on a rotary evaporator at $30{ }^{\circ} \mathrm{C}$. Due to its sensibility, the crude product was directly used for the next step. If desired

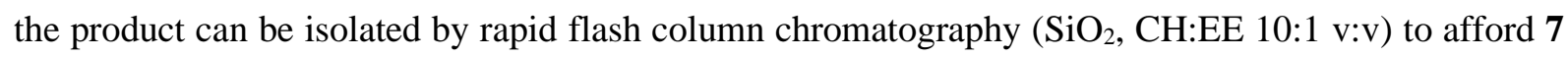
as a yellowish oil.

$\mathbf{R}_{f}=0.23\left(\mathrm{SiO}_{2}, \mathrm{CH}: \mathrm{EE} 10: 1 \mathrm{v}: \mathrm{v}\right) .{ }^{1} \mathbf{H}-\mathbf{N M R}\left(\mathbf{3 6 0} \mathbf{M H z}, \mathbf{C D C l}_{3}\right): \delta=10.71(\mathrm{~s}, 1 \mathrm{H}), 8.05\left(\mathrm{~d},{ }^{4} \boldsymbol{J}_{\mathrm{HH}}=2.1\right.$ $\mathrm{Hz}, 1 \mathrm{H}), 7.78\left(\mathrm{~d},{ }^{3} J_{\mathrm{HH}}=8.3 \mathrm{~Hz}, 1 \mathrm{H}\right), 7.71\left(\mathrm{dd},{ }^{3} J_{\mathrm{HH}}=8.3 \mathrm{~Hz},{ }^{4} J_{\mathrm{HH}}=2.1 \mathrm{~Hz}, 1 \mathrm{H}\right), 4.14(\mathrm{~s}, 2 \mathrm{H}), 1.39(\mathrm{~s}$, 6H) ppm. ${ }^{13} \mathbf{C}\left\{{ }^{1} \mathbf{H}\right\}$-NMR (91 MHz, $\left.\mathbf{C D C l}_{3}\right): \delta=191.3,159.6,137.4,135.9,131.6,131.0,128.6,125.9$, 79.5, 69.0, 28.5 (2x) ppm. IR (ATR, neat): $\tilde{v}=2967$ (w), 2928 (w), $2894(\mathrm{w}), 1687$ (s), 1644 (m), 1582 (m), 1563 (w), 1478 (w), 1381 (w), 1364 (w), 1351 (m), 1305 (m), 1284 (w), 1251 (w), 1212 (w), 1182 (m), 1129 (w), 1082 (w), 1046 (s), 987 (w), 963 (m), 922 (w), 881 (w), 865 (w), 836 (m), 819 (w), 712 (m), $677(\mathrm{~m}) \mathrm{cm}^{-1}$. MS (APCI): $\mathrm{m} / \mathrm{z}=282.0\left[\mathrm{C}_{12} \mathrm{H}_{12}{ }^{79} \mathrm{BrNO}_{2}+\mathrm{H}\right]^{+}$. HR-MS (ESI): calculated for $\mathrm{C}_{12} \mathrm{H}_{13}{ }^{79} \mathrm{BrNO}_{2}{ }^{+}[\mathrm{M}+\mathrm{H}]^{+}: \mathrm{m} / \mathrm{z}=282.01242$, found: 282.01244 (Dev.: $0.02 \mathrm{mu}$; $0.07 \mathrm{ppm}$ ). 


\subsection{3 (5-Bromo-2-(4,4-dimethyl-4,5-dihydrooxazol-2-yl)phenyl)methanol (8)}
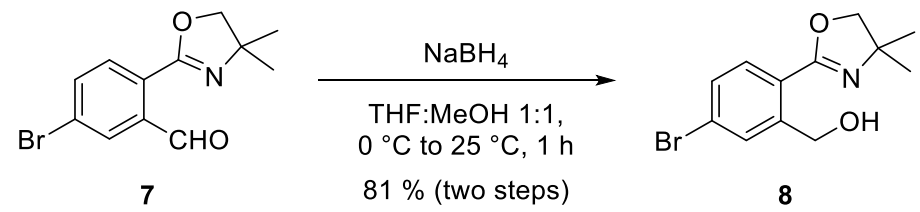

A modified literature procedure was used.[10] The crude benzaldehyde 7 (13.0 mmol, $1.00 \mathrm{eq})$ was dissolved in THF:MeOH (26 mL, 1:1 v:v, $0.5 \mathrm{M})$ and cooled to $0{ }^{\circ} \mathrm{C} . \mathrm{NaBH}_{4}(984 \mathrm{mg}, 26.0 \mathrm{mmol}$, $2.00 \mathrm{eq}$ ) was added and stirring was continued for two more hours while the mixture was allowed to warm slowly to $25^{\circ} \mathrm{C}$. Saturated $\mathrm{NH}_{4} \mathrm{Cl}$ solution $(20 \mathrm{~mL})$, ethyl acetate $(20 \mathrm{~mL})$ and water $(30 \mathrm{~mL})$ were added successively and the phases were separated. The aqueous layer was extracted with ethyl acetate $(4 \times 50 \mathrm{~mL})$, the combined organic phases were dried over anhydrous $\mathrm{NaSO}_{4}$, filtered and concentrated under reduced pressure. Purification by flash column chromatography $\left(\mathrm{SiO}_{2}, \mathrm{CH}: \mathrm{EE}\right.$ 10:1 v:v) afforded $\mathbf{8}$ (2.98 g, $10.5 \mathrm{mmol}, 81 \%$ over two steps $)$ as a colorless oil.

$\mathbf{R}_{f}=0.17\left(\mathrm{SiO}_{2}, \mathrm{CH}: \mathrm{EE} 10: 1 \mathrm{v}: \mathrm{v}\right) .{ }^{1} \mathbf{H}-\mathbf{N M R}\left(\mathbf{3 6 0} \mathbf{M H z}, \mathbf{C D C l}_{3}\right): \delta=7.71\left(\mathrm{~d},{ }^{3} \boldsymbol{J}_{\mathrm{HH}}=8.2 \mathrm{~Hz}, 1 \mathrm{H}\right), 7.51$ $\left(\mathrm{d},{ }^{4} J_{\mathrm{HH}}=2.1 \mathrm{~Hz}, 1 \mathrm{H}\right), 7.47\left(\mathrm{dd},{ }^{3} J_{\mathrm{HH}}=8.3 \mathrm{~Hz},{ }^{4} J_{\mathrm{HH}}=2.1 \mathrm{~Hz}, 1 \mathrm{H}\right), 6.65\left(\mathrm{t},{ }^{3} J_{\mathrm{HH}}=7.6 \mathrm{~Hz}, 1 \mathrm{H}\right), 4.59(\mathrm{~d}$, $\left.{ }^{3} J_{\mathrm{HH}}=7.6 \mathrm{~Hz}, 2 \mathrm{H}\right), 4.12(\mathrm{~s}, 2 \mathrm{H}), 1.39$ (s, 6H) ppm. ${ }^{13} \mathbf{C}\left\{{ }^{1} \mathbf{H}\right\}-\mathbf{N M R}\left(\mathbf{9 1} \mathbf{M H z}, \mathbf{C D C l}_{3}\right): \delta=161.6,144.0$, 133.4, 131.6, 130.8, 126.0, 125.7, 78.9, 68.2, 64.1, 28.5 (2x) ppm. IR (ATR, neat): $\tilde{v}=3270(\mathrm{w}), 2966$ (w), $2928(\mathrm{w}), 2895$ (w), 1636 (s), $1586(\mathrm{w}), 1561$ (w), 1462 (w), $1386(\mathrm{w}), 1352(\mathrm{~m}), 1308(\mathrm{~m}), 1284$ (w), 1186 (m), 1090 (m), 1054 (s), 1032 (s), 963 (m), 875 (w), 844 (m), 826 (m), 706 (m), 679 (m) $\mathrm{cm}^{-1}$. MS (APCI): $\mathrm{m} / \mathrm{z}=284.0\left[\mathrm{C}_{12} \mathrm{H}_{14}{ }^{79} \mathrm{BrNO}_{2}+\mathrm{H}\right]^{+}$. HR-MS (EI, $70 \mathrm{eV}$ ): calculated for $\mathrm{C}_{12} \mathrm{H}_{14}{ }^{79} \mathrm{BrNO}_{2}{ }^{+\cdot}[\mathrm{M}]^{+*}: \mathrm{m} / \mathrm{z}=283.02024$, found: 283.02011 (Dev.: $-0.13 \mathrm{mu} ;-0.47 \mathrm{ppm}$ ).

\subsubsection{2-(2-(Azidomethyl)-4-bromophenyl)-4,4-dimethyl-4,5-dihydrooxazole (9)}

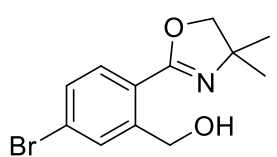

8

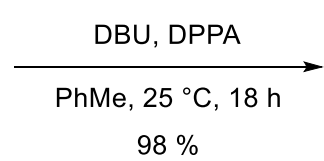

$98 \%$

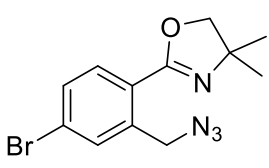

A modified literature procedure was used.[11] 8 (1.42 g, $5.00 \mathrm{mmol}, 1.00 \mathrm{eq})$ was dissolved in PhMe $(16.7 \mathrm{~mL}, 0.3 \mathrm{M})$ and DBU $(970 \mu \mathrm{L}, 6.50 \mathrm{mmol}, 1.30 \mathrm{eq})$ was added via syringe pump $(1.0 \mathrm{~mL} / \mathrm{min})$ at $25^{\circ} \mathrm{C}$. The mixture was stirred for ten minutes and DPPA $(1.29 \mathrm{~mL}, 6.00 \mathrm{mmol}, 1.20 \mathrm{eq})$ was added via syringe pump $(0.3 \mathrm{~mL} / \mathrm{min})$. After complete addition the suspension was stirred for 18 hours at $25^{\circ} \mathrm{C}$. Saturated $\mathrm{NH}_{4} \mathrm{Cl}$ solution $(50 \mathrm{~mL})$ and ethyl acetate $(100 \mathrm{~mL})$ were added and the phases separated. The aqueous phase was extracted with ethyl acetate $(3 \times 50 \mathrm{~mL})$ and the combined organic phases were dried over anhydrous $\mathrm{NaSO}_{4}$. After filtration the solvent was removed under reduced pressure and purification was conducted by flash column chromatography $\left(\mathrm{SiO}_{2}, \mathrm{CH}: \mathrm{EE} 15: 1 \mathrm{v}: \mathrm{v}\right)$ to provide 9 (1.52 $\mathrm{g}$, $4.92 \mathrm{mmol}, 98 \%$ ) as a colorless liquid. 
$\mathbf{R}_{f}=0.30\left(\mathrm{SiO}_{2}, \mathrm{CH}: \mathrm{EE} 15: 1 \mathrm{v}: \mathrm{v}\right)$. Mp.: around $0{ }^{\circ} \mathrm{C} .{ }^{1} \mathbf{H}-\mathbf{N M R}\left(\mathbf{3 6 0} \mathbf{M H z}, \mathbf{C D C l}_{\mathbf{3}}\right): \delta=7.75\left(\mathrm{~d},{ }^{3} \boldsymbol{J}_{\mathrm{HH}}=\right.$ $8.4 \mathrm{~Hz}, 1 \mathrm{H}), 7.62\left(\mathrm{~d},{ }^{4} J_{\mathrm{HH}}=2.1 \mathrm{~Hz}, 1 \mathrm{H}\right), 7.49\left(\mathrm{dd},{ }^{3} J_{\mathrm{HH}}=8.4 \mathrm{~Hz},{ }^{4} J_{\mathrm{HH}}=2.0 \mathrm{~Hz}, 1 \mathrm{H}\right), 4.87(\mathrm{~s}, 2 \mathrm{H}), 4.06$ (s, 2H), 1.37 (s, 6H) ppm. ${ }^{13} \mathbf{C}\left\{{ }^{1} \mathbf{H}\right\}-N M R\left(\mathbf{9 1} \mathbf{M H z}, \mathbf{C D C l}_{3}\right): \delta=160.4,138.4,132.3,131.7,131.1$, 125.6, 125.6, 78.6, 68.6, 52.8, 28.5 (2x) ppm. IR (ATR, neat): $\tilde{v}=2966$ (w), 2929 (w), 2892 (w), 2099 (s), 1642 (m), 1588 (w), 1564 (w), 1481 (w), 1350 (w), 1338 (w), 1304 (m), 1277 (m), 1212 (w), 1184 (m), 1090 (w), 1042 (s), 967 (w), 923 (w), 872 (w), 830 (m), 741 (w), 679 (w) cm-1. MS (APCI): m/z $=309.0\left[\mathrm{C}_{12} \mathrm{H}_{13}{ }^{79} \mathrm{BrN}_{4} \mathrm{O}+\mathrm{H}\right]^{+}$. HR-MS (ESI): calculated for $\mathrm{C}_{12} \mathrm{H}_{13}{ }^{79} \mathrm{BrN}_{4} \mathrm{O}^{+}[\mathrm{M}+\mathrm{H}]^{+}: \mathrm{m} / \mathrm{z}=309.03455$, found: 309.03499 (Dev.: $0.44 \mathrm{mu} ; 1.43 \mathrm{ppm}$ ).

\subsubsection{2-(Azidomethyl)-4-bromobenzaldehyde (3)}

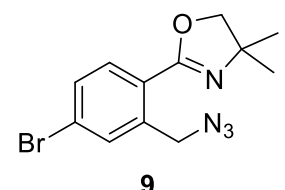

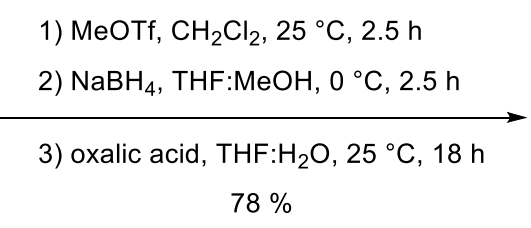

$78 \%$

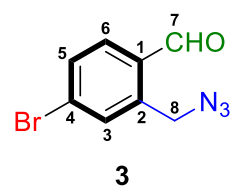

3

A modified literature procedure was used.[12] 9 (1.86 g, $6.00 \mathrm{mmol}, 1.00 \mathrm{eq})$ was dissolved in $\mathrm{CH}_{2} \mathrm{Cl}_{2}$ $(20.0 \mathrm{~mL}, 0.3 \mathrm{M})$ and MeOTf $(1.31 \mathrm{~mL}, 12.0 \mathrm{mmol}, 2.00 \mathrm{eq})$ was added via syringe in one portion at $25^{\circ} \mathrm{C}$. After stirring for two and a half hours at the same temperature the solution was cooled to $0{ }^{\circ} \mathrm{C}$. A solution of $\mathrm{NaBH}_{4}(454 \mathrm{mg}, 12.0 \mathrm{mmol}, 2.00 \mathrm{eq})$ in THF:MeOH (20.0 mL, 4:1 v:v, $\left.0.3 \mathrm{M}\right)$ was added via syringe within 20 minutes and stirring was continued at $0{ }^{\circ} \mathrm{C}$ for another two and a half hours. Saturated $\mathrm{NH}_{4} \mathrm{Cl}$ solution $(20 \mathrm{~mL})$, water $(20 \mathrm{~mL})$ and $\mathrm{CH}_{2} \mathrm{Cl}_{2}(40 \mathrm{~mL})$ were added and the phases separated. The aqueous layer was extracted with $\mathrm{CH}_{2} \mathrm{Cl}_{2}(3 \times 50 \mathrm{~mL})$ and the combined organic phases were dried over anhydrous $\mathrm{Na}_{2} \mathrm{SO}_{4}$. After filtration and solvent evaporation under reduced pressure, the residue was redissolved in THF: $\mathrm{H}_{2} \mathrm{O}(20.0 \mathrm{~mL}, 4: 1 \mathrm{v}: \mathrm{v}, 0.3 \mathrm{M})$. Oxalic acid dihydrate $(1.51 \mathrm{~g}$, $12.0 \mathrm{mmol}, 2.00 \mathrm{eq}$ ) was added at $25^{\circ} \mathrm{C}$ and the mixture was stirred for 18 hours at the same temperature. Saturated $\mathrm{NaHCO}_{3}$ solution $(20 \mathrm{~mL})$, water $(20 \mathrm{~mL})$ and $\mathrm{CH}_{2} \mathrm{Cl}_{2}(40 \mathrm{~mL})$ were added and the phases separated. The aqueous layer was extracted with $\mathrm{CH}_{2} \mathrm{Cl}_{2}(4 \times 40 \mathrm{~mL})$ and the combined organic phases were dried over anhydrous $\mathrm{Na}_{2} \mathrm{SO}_{4}$. Filtration and solvent evaporation under reduced pressure and purification by flash column chromatography ( $\left.\mathrm{SiO}_{2}, \mathrm{CH}: \mathrm{EE} 20: 1 \mathrm{v}: \mathrm{v}\right)$ afforded $\mathbf{3}$ (1.12 $\mathrm{g}$, $4.67 \mathrm{mmol}, 78 \%$ ) as an off-white solid.

$\mathbf{R}_{f}=0.24\left(\mathrm{SiO}_{2}, \mathrm{CH}: \mathrm{EE} 20: 1 \mathrm{v}: \mathrm{v}\right)$. Mp.: $47-49{ }^{\circ} \mathrm{C} .{ }^{1} \mathbf{H}-\mathbf{N M R}\left(\mathbf{3 6 0} \mathbf{M H z}, \mathbf{C D C l}_{3}\right): \delta=10.07\left(\mathrm{~d},{ }^{5} \boldsymbol{J}_{\mathrm{HH}}=\right.$ $0.1 \mathrm{~Hz} 1 \mathrm{H}, \mathrm{CHO}), 7.76-7.73$ (m, 1H, H-3), $7.73-7.66$ (m, 2H, H-5, H-6), 4.88 (s, 2H, CH H $_{2} \mathrm{ppm}$. ${ }^{13} \mathbf{C}\left\{{ }^{1} \mathbf{H}\right\}$-NMR (91 MHz, $\left.\mathbf{C D C l}_{3}\right): \delta=192.0(\mathrm{C}-7), 139.3(\mathrm{C}-2), 135.9(\mathrm{C}-6), 132.4(\mathrm{C}-3), 132.1(\mathrm{C}-$ 1), 131.8 (C-5), 129.8 (C-4), 51.7 (C-8) ppm. IR (ATR, neat): $\tilde{v}=3098$ (w), 2928 (w), 2849 (w), 2751 (w), 2114 (m), 1687 (m), 1593 (m), 1556 (m), 1426 (m), 1378 (w), 1338 (m), 1292 (m), 1223 (w), 1197 (m), 1086 (m), 979 (w), 933 (m), 885 (m), 872 (m), 828 (w), 802 (s), 701 (w), 688 (m) cm $\mathrm{cm}^{-1}$. MS (ESI): $\mathrm{m} / \mathrm{z}=262.1\left[\mathrm{C}_{8} \mathrm{H}_{6}{ }^{79} \mathrm{BrN}_{3} \mathrm{O}+\mathrm{Na}\right]^{+}$. HR-MS (ESI): calculated for $\mathrm{C}_{8} \mathrm{H}_{6}{ }^{79} \mathrm{BrN}_{3} \mathrm{O}^{+}[\mathrm{M}+\mathrm{Na}]^{+}: \mathrm{m} / \mathrm{z}$ 
$=261.95864$, found: 261.95890 (Dev.: $0.26 \mathrm{mu} ; 0.97 \mathrm{ppm}$ ). The analytical data are in accordance with the literature.[13]

\subsection{Preparation of 4-(Azidomethyl)-2-bromobenzaldehyde (4)}

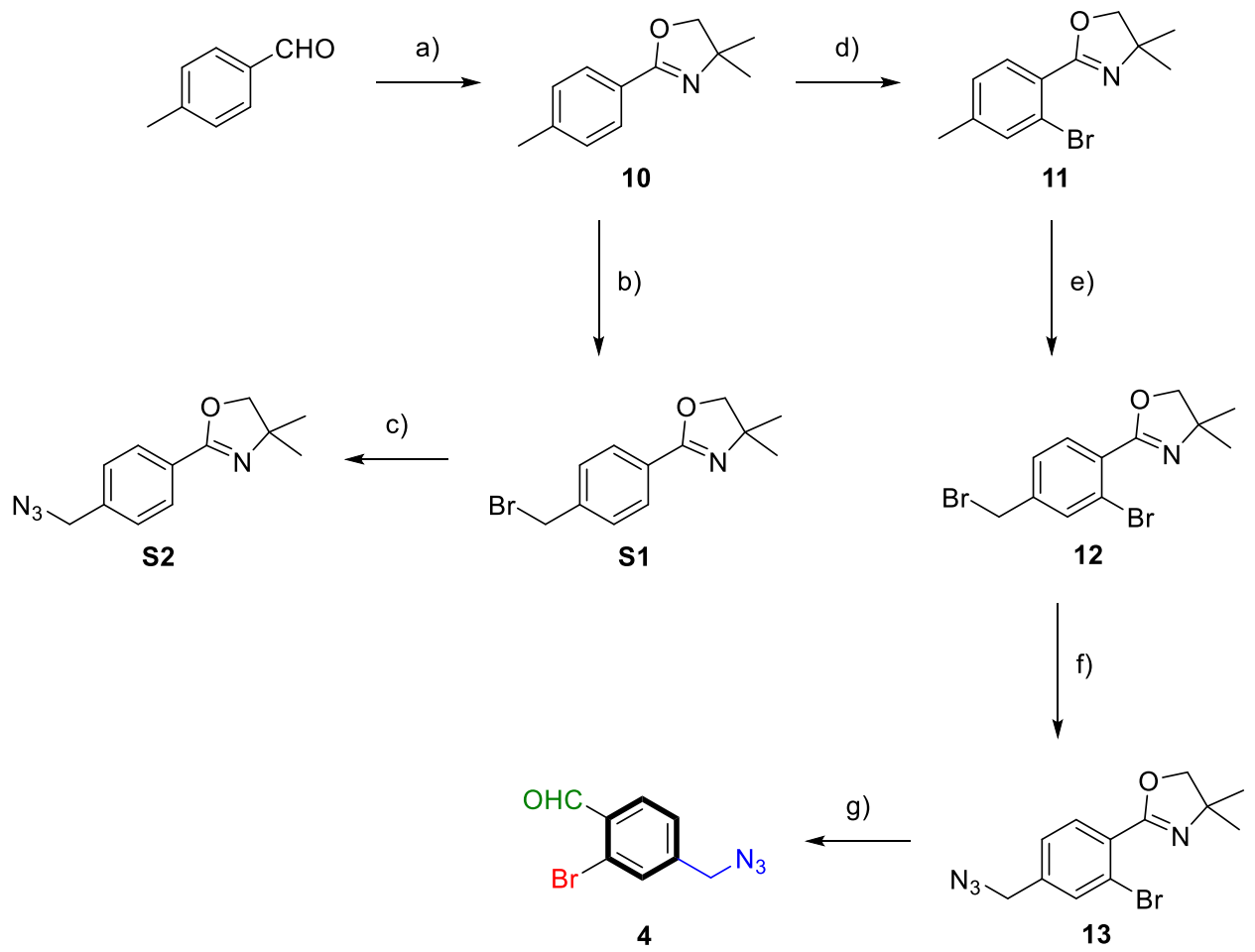

Reaction conditions: a) 1) 2-amino-2-methylpropan-1-ol, $4 \AA \mathrm{AS}, \mathrm{CH}_{2} \mathrm{Cl}_{2}, 25^{\circ} \mathrm{C}, 18 \mathrm{~h}$; 2) $\mathrm{NBS}, \mathrm{CH}_{2} \mathrm{Cl}_{2}, 25^{\circ} \mathrm{C}, 4 \mathrm{~h}, 98 \%$; b) NBS, AIBN, $\mathrm{CCl}_{4}, 100{ }^{\circ} \mathrm{C}, 5 \mathrm{~h}, 78 \%$; c) $\mathrm{NaN}_{3}$, DMF, $25^{\circ} \mathrm{C}, 4 \mathrm{~h}, 90 \%$; d) 1) TMPMgCl$\left.\cdot \mathrm{LiCl}, \mathrm{THF}, 25^{\circ} \mathrm{C}, 4 \mathrm{~h} ; 2\right)\left(\mathrm{CBrCl}_{2}\right)_{2}$, THF, $0{ }^{\circ} \mathrm{C}$ to $25{ }^{\circ} \mathrm{C}, 10 \mathrm{~h}, 76 \%$; e) NBS, AIBN, CCl $4,100{ }^{\circ} \mathrm{C}, 7 \mathrm{~h}, 66 \%$; f) NaN3, DMF, $25{ }^{\circ} \mathrm{C}, 4 \mathrm{~h}, 99 \%$; g) 1) MeOTf, $\mathrm{CH}_{2} \mathrm{Cl}_{2}, 25^{\circ} \mathrm{C}, 2.5 \mathrm{~h}$; 2) $\mathrm{NaBH}_{4}$, THF:MeOH 4:1 v:v, $\left.0^{\circ} \mathrm{C}, 2.5 \mathrm{~h} ; 3\right)$ oxalic acid, THF: $\mathrm{H}_{2} \mathrm{O}$ 4:1 v:v, $25^{\circ} \mathrm{C}, 20 \mathrm{~h}, 85 \%$. Overall yield: $41 \%$ (5 steps).

\subsubsection{4,4-Dimethyl-2-(p-tolyl)-4,5-dihydrooxazole (10)}<smiles>Cc1ccc(C=O)cc1</smiles>

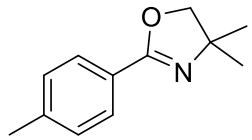

10

A modified literature procedure was used.[8] $p$-Tolualdehyde (8.41 g, $70.0 \mathrm{mmol}, 1.00 \mathrm{eq})$ and 2-amino2-methylpropan-1-ol (12.5 g, $140 \mathrm{mmol}, 2.00 \mathrm{eq})$ were dissolved in $\mathrm{CH}_{2} \mathrm{Cl}_{2}(175 \mathrm{~mL}, 0.4 \mathrm{M})$ and $4 \AA$ AS $(25.0 \mathrm{~g})$ was added. After 18 hours of slowly stirring at $25^{\circ} \mathrm{C}$ NBS $(24.9 \mathrm{~g}, 140.0 \mathrm{mmol}$, $2.00 \mathrm{eq}$ ) was added in one portion and stirring was continued for another four hours at $25^{\circ} \mathrm{C}$. All solids were filtered off and the organic phase was washed with saturated $\mathrm{NaHCO}_{3}$ solution $(3 \times 100 \mathrm{~mL})$. The combined aqueous phases were extracted with $\mathrm{CH}_{2} \mathrm{Cl}_{2}(2 \times 100 \mathrm{~mL})$. All organic phases were washed with saturated $\mathrm{NaS}_{2} \mathrm{O}_{3}$ solution $(100 \mathrm{~mL})$ and the aqueous phase was extracted with $\mathrm{CH}_{2} \mathrm{Cl}_{2}(50 \mathrm{~mL})$. 
The combined organic phases were dried over anhydrous $\mathrm{MgSO}_{4}$, filtered and the solvent was removed under reduced pressure. Purification by flash column chromatography $\left(\mathrm{SiO}_{2}, \mathrm{CH}: \mathrm{EE} 10: 1\right.$ to 5:1 v:v) afforded $10(13.0 \mathrm{~g}, 68.7 \mathrm{mmol}, 98 \%)$ as a pale brown solid with a characteristic nougat odor.

$\mathbf{R}_{f}=0.16\left(\mathrm{SiO}_{2}, \mathrm{CH}: \mathrm{EE}\right.$ 10:1 v:v). Mp.: $42-44{ }^{\circ} \mathrm{C} .{ }^{1} \mathbf{H}-\mathbf{N M R}\left(\mathbf{3 6 0} \mathbf{M H z}, \mathbf{C D C l}_{3}\right): \delta=7.81\left(\mathrm{~d},{ }^{3} \boldsymbol{J}_{\mathrm{HH}}=\right.$ $7.7 \mathrm{~Hz}, 2 \mathrm{H}), 7.19\left(\mathrm{~d},{ }^{3} \mathrm{~J}_{\mathrm{HH}}=7.8 \mathrm{~Hz}, 2 \mathrm{H}\right), 4.07(\mathrm{~s}, 2 \mathrm{H}), 2.37$ (s, 3H), $1.36(\mathrm{~s}, 6 \mathrm{H}) \mathrm{ppm} .{ }^{13} \mathbf{C}\left\{{ }^{1} \mathbf{H}\right\}-\mathbf{N M R}$ (91 MHz, $\left.\mathbf{C D C l}_{3}\right): \delta=162.2,141.6,129.1$ (2x), 128.3 (2x), 125.3, 79.1, 67.6, 28.5 (2x), 21.6 ppm. IR (ATR, neat): $\tilde{v}=2970(\mathrm{w}), 2924$ (w), 2894 (w), 1642 (m), 1510 (m), 1463 (w), 1409 (w), 1364 (w), 1353 (m), 1316 (m), 1302 (m), 1250 (w), 1193 (w), 1173 (m), 1065 (s), 1019 (m), 992 (w), 965 (m), $917(\mathrm{~m}), 871(\mathrm{w}), 829(\mathrm{~s}), 727(\mathrm{~s}), 682(\mathrm{~s}) \mathrm{cm}^{-1}$. MS (APCI): $\mathrm{m} / \mathrm{z}=190.1[\mathrm{M}+\mathrm{H}]^{+}$. The analytical data are in accordance with the literature.[14]

\subsubsection{2-(4-(Bromomethyl)phenyl)-4,4-dimethyl-4,5-dihydrooxazole (S1)}

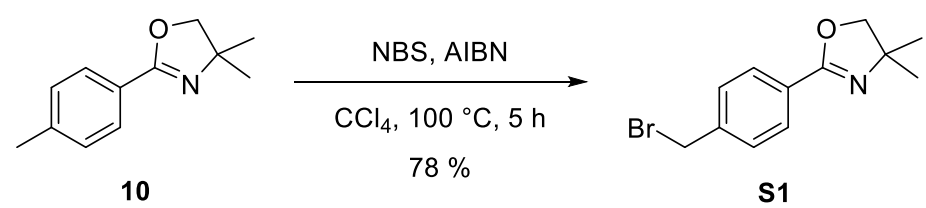

A modified literature procedure was used.[15] $10(568 \mathrm{mg}, 3.00 \mathrm{mmol}, 1.00 \mathrm{eq})$ was dissolved in $\mathrm{CCl}_{4}$ $(10 \mathrm{~mL}, 0.3 \mathrm{M})$ and NBS (534 mg, $3.00 \mathrm{mmol}, 1.00 \mathrm{eq})$ was added in one portion. AIBN (24.3 mg, $150 \mu \mathrm{mol}, 0.05 \mathrm{eq}$ ) was added and the mixture was heated to $100{ }^{\circ} \mathrm{C}$ (oil bath temperature) for five hours. After cooling to $25{ }^{\circ} \mathrm{C}$ the mixture was directly filtered over anhydrous $\mathrm{Na}_{2} \mathrm{SO}_{4}$ and the solids were washed with $\mathrm{CCl}_{4}(20 \mathrm{~mL})$. The solvent was evaporated under reduced pressure and purification

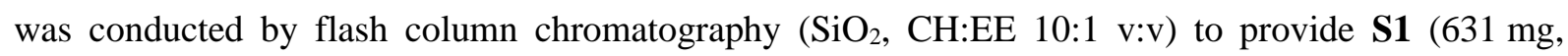
$2.35 \mathrm{mmol}, 78 \%$ ) as a colorless solid.

$\mathbf{R}_{f}=0.11\left(\mathrm{SiO}_{2}\right.$, CH:EE 10:1 v:v). Mp.: $77-79{ }^{\circ} \mathrm{C} .{ }^{1} \mathbf{H}-\mathrm{NMR}\left(\mathbf{3 6 0} \mathbf{~ M H z}, \mathbf{C D C l}_{3}\right): \delta=7.94-7.87$ (AA'XX', 2H), $7.45-7.39$ (AA'XX', 2H), 4.49 (s, 2H), 4.11 (s, 2H), 1.38 (s, 6H) ppm. ${ }^{13}{ }^{13}\left\{{ }^{1} \mathbf{H}\right\}-\mathbf{N M R}$ (91 MHz, $\left.\mathbf{C D C l}_{3}\right): \delta=161.6,140.9,129.1$ (2x), 128.8 (2x), 128.2, 79.3, 67.8, 32.8, 28.6 (2x) ppm. IR (ATR, neat): $\tilde{v}=2965$ (w), 2926 (w), 2898 (w), 1644 (m), 1511 (w), 1458 (w), 1415 (m), 1357 (m), 1320 (m), 1299 (m), 1228 (w), 1194 (w), 1177 (m), 1068 (s), 1018 (m), 987 (w), 960 (m), 918 (w), 870 (w), $849(\mathrm{~m}), 835(\mathrm{~m}), 819(\mathrm{~m}), 747(\mathrm{w}), 688(\mathrm{~s}) \mathrm{cm}^{-1}$. MS (APCI): $\mathrm{m} / \mathrm{z}=267.9\left[\mathrm{C}_{12} \mathrm{H}_{14}{ }^{79} \mathrm{BrNO}+\mathrm{H}\right]^{+}$. HR-MS (EI, $70 \mathrm{eV}$ ): calculated for $\mathrm{C}_{12} \mathrm{H}_{14}{ }^{79} \mathrm{BrNO}^{+}[\mathrm{M}]^{+}: \mathrm{m} / \mathrm{z}=267.02533$, found: 267.02514 (Dev.: $-0.19 \mathrm{mu} ;-0.70 \mathrm{ppm})$. The analytical data are in accordance with the literature.[16] 


\subsubsection{2-(4-(Azidomethyl)phenyl)-4,4-dimethyl-4,5-dihydrooxazole (S2)}<smiles>CC1(C)COC(c2ccc(CBr)cc2)=N1</smiles>

S1

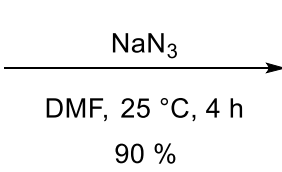

$90 \%$

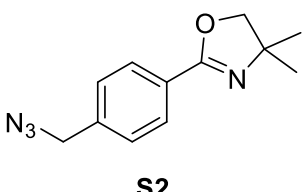

S2

A modified literature procedure was used.[13] $\mathbf{S 1}$ (992 mg, $3.70 \mathrm{mmol}, 1.00 \mathrm{eq}$ ) was dissolved in DMF $(12.3 \mathrm{~mL}, 0.3 \mathrm{M})$ and $\mathrm{NaN}_{3}(481 \mathrm{mg}, 7.40 \mathrm{mmol}, 2.00 \mathrm{eq})$ was added in one portion. After stirring for four hours at $25{ }^{\circ} \mathrm{C}$ water $(20 \mathrm{~mL})$ and $\mathrm{CH}_{2} \mathrm{Cl}_{2}(50 \mathrm{~mL})$ were added and the phases separated. The aqueous phase was extracted with $\mathrm{CH}_{2} \mathrm{Cl}_{2}(6 \times 50 \mathrm{~mL})$ and the combined organic phases were dried over anhydrous $\mathrm{Na}_{2} \mathrm{SO}_{4}$, filtered and the solvent was removed under reduced pressure. Purification by flash column chromatography ( $\mathrm{SiO}_{2}, \mathrm{CH}: \mathrm{EE} 10: 1$ to $\left.5: 1 \mathrm{v}: \mathrm{v}\right)$ provided $\mathbf{S 2}(771 \mathrm{mg}, 3.35 \mathrm{mmol}, 90 \%)$ as a colorless liquid.

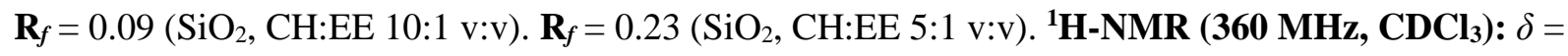
7.98 - 7.92 (AA'XX', 2H), 7.39 - 7.32 (AA'XX', 2H), 4.38 (s, 2H), 4.11 (s, 2H), 1.38 (s, 6H) ppm. ${ }^{13} \mathbf{C}\left\{{ }^{1} \mathbf{H}\right\}$-NMR (91 MHz, $\left.\mathbf{C D C l}_{3}\right): \delta=161.3,138.2,128.5$ (2x), 127.8, 127.7 (2x), 78.9, 67.4, 54.1, 28.2 (2x) ppm. IR (ATR, neat): $\tilde{v}=2967$ (w), 2928 (w), 2893 (w), 2094 (s), 1647 (s), 1513 (w), 1462 (w), 1417 (m), 1352 (m), 1316 (m), 1297 (m), 1247 (m), 1179 (m), 1064 (s), 1019 (m), 991 (w), 965 (m), 920 (w), $844(\mathrm{~m}), 802(\mathrm{~m}), 730(\mathrm{~m}), 693(\mathrm{~m}) \mathrm{cm}^{-1}$. MS (APCI): m/z = $231.0\left[\mathrm{C}_{12} \mathrm{H}_{14} \mathrm{~N}_{4} \mathrm{O}+\mathrm{H}\right]^{+}$. HR-MS (EI, $70 \mathrm{eV}$ ): calculated for $\mathrm{C}_{12} \mathrm{H}_{14} \mathrm{~N}_{4} \mathrm{O}^{+\cdot}[\mathrm{M}]^{+\cdot}: \mathrm{m} / \mathrm{z}=230.11621$, found: 230.11607 (Dev.: $-0.14 \mathrm{mu}$; $-0.62 \mathrm{ppm})$.

\subsubsection{2-(2-Bromo-4-methylphenyl)-4,4-dimethyl-4,5-dihydrooxazole (11)}

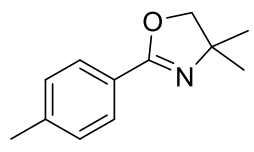

10

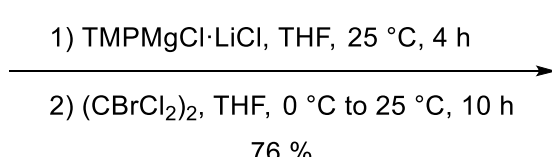

$76 \%$

A modified literature procedure was used.[9] A heat gun-dried and nitrogen-flushed Schlenk flask, equipped with a magnetic stirring bar and a rubber septum was charged with $\mathbf{1 1}(1.51 \mathrm{~g}, 8.00 \mathrm{mmol}$, $1.00 \mathrm{eq}$ ) and evacuated for half an hour. The flask was flushed with nitrogen and anhydrous THF $(20.0 \mathrm{~mL}, 0.4 \mathrm{M})$ was added. Addition of TMPMgCl$\cdot \mathrm{LiCl}(24.0 \mathrm{~mL}, 24.0 \mathrm{mmol}, 3.00 \mathrm{eq}, 1.00 \mathrm{M}$ in THF) via syringe within two minutes at $25{ }^{\circ} \mathrm{C}$ was followed by stirring for four hours at the same conditions. 1,2-Dibromotetrachloroethane $(7.82 \mathrm{~g}, 24.0 \mathrm{mmol}, 3.00 \mathrm{eq})$ was diluted in anhydrous THF $(12 \mathrm{~mL})$ and added via syringe pump $(0.5 \mathrm{~mL} / \mathrm{min})$ to the dark red solution at $0{ }^{\circ} \mathrm{C}$. After complete addition the mixture was allowed to warm to $25^{\circ} \mathrm{C}$ and stirred for a total of ten hours. Saturated $\mathrm{NH}_{4} \mathrm{Cl}$ solution $(20 \mathrm{~mL})$, water $(50 \mathrm{~mL})$ and $\mathrm{CH}_{2} \mathrm{Cl}_{2}(50 \mathrm{~mL})$ were added and the phases separated. The aqueous phase was extracted with $\mathrm{CH}_{2} \mathrm{Cl}_{2}(3 \times 50 \mathrm{~mL})$ and the combined organic phases were dried over 
anhydrous $\mathrm{Na}_{2} \mathrm{SO}_{4}$. Filtration, solvent evaporation under reduced pressure and purification by flash

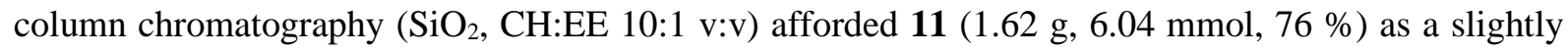
yellow liquid.

$\mathbf{R}_{\boldsymbol{f}}=0.15\left(\mathrm{SiO}_{2}, \mathrm{CH}: \mathrm{EE} 10: 1 \mathrm{v}: \mathrm{v}\right) .{ }^{1} \mathbf{H}-\mathbf{N M R}\left(\mathbf{3 6 0} \mathbf{M H z}, \mathbf{C D C l}_{3}\right): \delta=7.54\left(\mathrm{~d},{ }^{3} \boldsymbol{J}_{\mathrm{HH}}=7.8 \mathrm{~Hz}, 1 \mathrm{H}\right), 7.44$ $\left(\mathrm{d},{ }^{4} J_{\mathrm{HH}}=0.6 \mathrm{~Hz}, 1 \mathrm{H}\right), 7.12\left(\mathrm{dd},{ }^{3} J_{\mathrm{HH}}=7.9 \mathrm{~Hz},{ }^{4} J_{\mathrm{HH}}=0.8 \mathrm{~Hz}, 1 \mathrm{H}\right), 4.11(\mathrm{~s}, 2 \mathrm{H}), 2.34(\mathrm{~s}, 3 \mathrm{H}), 1.40(\mathrm{~s}$, 6H) ppm. ${ }^{13} \mathbf{C}\left\{{ }^{1} \mathbf{H}\right\}$-NMR (91 MHz, $\left.\mathbf{C D C l}_{3}\right): \delta=161.5,141.9,133.9,130.8,127.6,127.0,121.3,79.0$, 67.7, 28.0, 20.8 ppm. IR (ATR, neat): $\tilde{v}=2965$ (w), 2926 (w), 2889 (w), 1651 (m), 1604 (w), 1493 (w), 1460 (w), 1390 (w), 1363 (w), 1349 (m), 1308 (m), 1264 (w), 1248 (w), 1188 (w), 1084 (s), 1027 (s), $963(\mathrm{~m}), 920(\mathrm{w}), 877$ (w), $827(\mathrm{~m}), 812(\mathrm{~m}), 736(\mathrm{~m}), 682(\mathrm{w}), 671(\mathrm{~m}) \mathrm{cm}^{-1}$. MS (APCI): m/z = $267.9\left[\mathrm{C}_{12} \mathrm{H}_{14}{ }^{79} \mathrm{BrNO}+\mathrm{H}\right]^{+}$. HR-MS (EI, 70 eV): calculated for $\mathrm{C}_{12} \mathrm{H}_{14}{ }^{79} \mathrm{BrNO}^{+\cdot}[\mathrm{M}]^{+\cdot}: \mathrm{m} / \mathrm{z}=267.02533$, found: 267.02509 (Dev.: $-0.24 \mathrm{mu} ;-0.89 \mathrm{ppm}$ ). The analytical data are in accordance with the literature.[17]

\subsubsection{2-(2-Bromo-4-(bromomethyl)phenyl)-4,4-dimethyl-4,5-dihydrooxazole (12)}

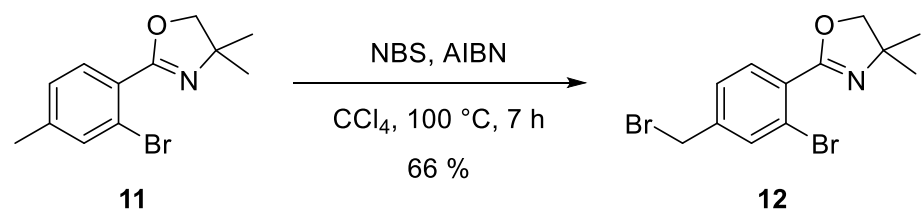

A modified literature procedure was used.[15] $11(536 \mathrm{mg}, 2.00 \mathrm{mmol}, 1.00 \mathrm{eq})$ was dissolved in $\mathrm{CCl}_{4}$ $(6.67 \mathrm{~mL}, 0.3 \mathrm{M})$ and NBS (356 mg, $2.00 \mathrm{mmol}, 1.00 \mathrm{eq})$ was added in one portion. AIBN (16.4 mg, $100 \mu \mathrm{mol}, 0.05 \mathrm{eq}$ ) was added and the mixture was heated to $100{ }^{\circ} \mathrm{C}$ for seven hours. After cooling to $25^{\circ} \mathrm{C}$ the mixture was directly filtered over anhydrous $\mathrm{Na}_{2} \mathrm{SO}_{4}$ and the solids were washed with $\mathrm{CCl}_{4}$ $(30 \mathrm{~mL})$. The solvent was evaporated under reduced pressure and purification was conducted by flash column chromatography ( $\left.\mathrm{SiO}_{2}, \mathrm{CH}: \mathrm{EE} 10: 1 \mathrm{v}: \mathrm{v}\right)$ to provide $12(457 \mathrm{mg}, 1.32 \mathrm{mmol}, 66 \%)$ as a colorless liquid. Upon standing the product slowly solidified to form a colorless solid.

$\mathbf{R}_{\boldsymbol{f}}=0.11\left(\mathrm{SiO}_{2}, \mathrm{CH}: \mathrm{EE}\right.$ 10:1 v:v). Mp.: $70-72{ }^{\circ} \mathrm{C} .{ }^{1} \mathbf{H}-\mathrm{NMR}\left(\mathbf{3 6 0} \mathbf{M H z}, \mathbf{C D C l}_{3}\right): \delta=7.65\left(\mathrm{~d},{ }^{4} \boldsymbol{J}_{\mathrm{HH}}=\right.$ $1.8 \mathrm{~Hz}, 1 \mathrm{H}), 7.63\left(\mathrm{~d},{ }^{3} J_{\mathrm{HH}}=7.9 \mathrm{~Hz}, 1 \mathrm{H}\right), 7.34\left(\mathrm{dd},{ }^{3} J_{\mathrm{HH}}=7.9 \mathrm{~Hz},{ }^{4} J_{\mathrm{HH}}=1.8 \mathrm{~Hz}, 1 \mathrm{H}\right), 4.41(\mathrm{~s}, 2 \mathrm{H}), 4.13$ (s, 2H), 1.40 (s, 6H) ppm. ${ }^{13} \mathbf{C}\left\{{ }^{1} \mathbf{H}\right\}-N M R\left(\mathbf{9 1} \mathbf{~ M H z}, \mathbf{C D C l}_{3}\right): \delta=161.3,141.5,134.2,131.7,130.2$, 127.8, 122.1, 79.5, 68.3, 31.3, 28.4 ppm. IR (ATR, neat): $\tilde{v}=2969$ (w), 2930 (w), $2892(\mathrm{w}), 1652(\mathrm{~m})$, 1491 (w), 1459 (w), 1397 (w), 1383 (w), 1363 (w), 1346 (m), 1308 (m), 1270 (w), 1216 (m), 1194 (m), 1170 (w), 1083 (s), 1028 (s), 991 (w), 968 (m), 939 (w), 912 (m), 884 (m), 847 (m), 834 (m), 816 (m), $694(\mathrm{~s}), 673(\mathrm{~m}) \mathrm{cm}^{-1}$. MS (APCI): $\mathrm{m} / \mathrm{z}=345.9\left[\mathrm{C}_{12} \mathrm{H}_{13}{ }^{79} \mathrm{Br}_{2} \mathrm{NO}+\mathrm{H}\right]^{+}$. HR-MS (EI, 70 eV): calculated for $\mathrm{C}_{12} \mathrm{H}_{13}{ }^{79} \mathrm{Br}_{2} \mathrm{NO}^{+\cdot}[\mathrm{M}]^{+}: \mathrm{m} / \mathrm{z}=344.93584$, found: 344.93583 (Dev.: $-0.01 \mathrm{mu} ;-0.03 \mathrm{ppm}$ ). 


\subsubsection{2-(4-(Azidomethyl)-2-bromophenyl)-4,4-dimethyl-4,5-dihydrooxazole (13)}

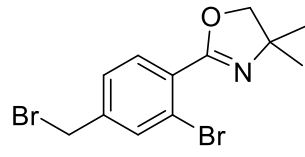

12

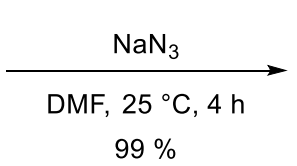

$99 \%$

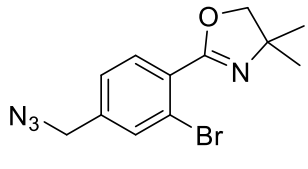

13

A modified literature procedure was used.[13] $12(521 \mathrm{mg}, 1.50 \mathrm{mmol}, 1.00 \mathrm{eq})$ was dissolved in DMF (5.00 mL, $0.3 \mathrm{M})$ and $\mathrm{NaN}_{3}(195 \mathrm{mg}, 3.00 \mathrm{mmol}, 2.00 \mathrm{eq})$ was added in one portion. After stirring for four hours at $25{ }^{\circ} \mathrm{C}$ water $(20 \mathrm{~mL})$ and $\mathrm{CH}_{2} \mathrm{Cl}_{2}(50 \mathrm{~mL})$ were added and the phases separated. The aqueous phase was extracted with $\mathrm{CH}_{2} \mathrm{Cl}_{2}(6 \times 50 \mathrm{~mL})$ and the combined organic phases were dried over anhydrous $\mathrm{Na}_{2} \mathrm{SO}_{4}$, filtered and the solvent was removed under reduced pressure. Purification by flash column chromatography $\left(\mathrm{SiO}_{2}, \mathrm{CH}: \mathrm{EE} 10: 1\right.$ to $\left.5: 1 \mathrm{v}: \mathrm{v}\right)$ provided $13(457 \mathrm{mg}, 1.48 \mathrm{mmol}, 99 \%)$ as a colorless liquid.

$\mathbf{R}_{\boldsymbol{f}}=0.10\left(\mathrm{SiO}_{2}, \mathrm{CH}: \mathrm{EE} \mathrm{10:1} \mathrm{v:v).} \mathbf{R}_{\boldsymbol{f}}=0.24\left(\mathrm{SiO}_{2}, \mathrm{CH}: \mathrm{EE}\right.\right.$ 5:1 v:v). ${ }^{1} \mathbf{H}-\mathbf{N M R}\left(\mathbf{3 6 0} \mathbf{M H z}, \mathbf{C D C l}_{\mathbf{3}}\right): \delta=$ $7.67\left(\mathrm{~d},{ }^{3} J_{\mathrm{HH}}=7.9 \mathrm{~Hz}, 1 \mathrm{H}\right), 7.59\left(\mathrm{~d},{ }^{4} J_{\mathrm{HH}}=1.7 \mathrm{~Hz}, 1 \mathrm{H}\right), 7.28\left(\mathrm{dd},{ }^{3} J_{\mathrm{HH}}=7.9 \mathrm{~Hz},{ }^{4} J_{\mathrm{HH}}=1.7 \mathrm{~Hz}, 1 \mathrm{H}\right)$, 4.36 (s, 2H), 4.14 (s, 2H), 1.41 (s, 6H) ppm. ${ }^{13} \mathbf{C}\left\{{ }^{1} \mathbf{H}\right\}-\mathbf{N M R}$ (91 MHz, $\left.\mathbf{C D C l}_{3}\right): \delta=161.4,139.4,133.1$, 131.8, 130.2, 126.6, 122.3 , 79.6, 68.3, 53.7, 28.4 ppm. IR (ATR, neat): $\tilde{v}=2966$ (w), 2928 (w), 2891 (w), 2095 (s), 1652 (m), 1604 (w), 1492 (w), 1461 (w), 1397 (m), 1350 (m), 1338 (m), 1308 (m), 1280 (m), 1247 (m), 1189 (m), 1086 (s), 1028 (s), 961 (m), 920 (m), 888 (m), 826 (m), 813 (m), 738 (w), 686 (m) $\mathrm{cm}^{-1}$. MS (APCI): $\mathrm{m} / \mathrm{z}=309.0\left[\mathrm{C}_{12} \mathrm{H}_{13}{ }^{79} \mathrm{BrN}_{4} \mathrm{O}+\mathrm{H}\right]^{+}$. HR-MS (EI, $70 \mathrm{eV}$ ): calculated for $\mathrm{C}_{12} \mathrm{H}_{13}{ }^{79} \mathrm{BrN}_{4} \mathrm{O}^{+\cdot}[\mathrm{M}]^{+\cdot}: \mathrm{m} / \mathrm{z}=308.02672$, found: 308.02707 (Dev.: $0.35 \mathrm{mu} ; 1.12 \mathrm{ppm}$ ).

\subsubsection{4-(Azidomethyl)-2-bromobenzaldehyde (4)}

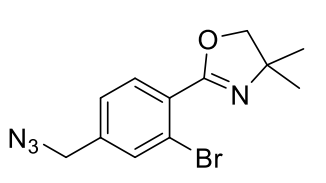

13

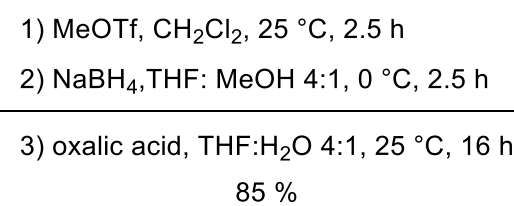

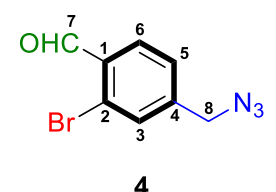

4

A modified literature procedure was used.[12] 13 (928 mg, $3.00 \mathrm{mmol}, 1.00 \mathrm{eq})$ was dissolved in $\mathrm{CH}_{2} \mathrm{Cl}_{2}$ $(10.0 \mathrm{~mL}, 0.3 \mathrm{M})$ and MeOTf $(656 \mu \mathrm{L}, 6.00 \mathrm{mmol}, 2.00 \mathrm{eq})$ was added via syringe in one portion at $25^{\circ} \mathrm{C}$. After stirring for two and a half hours at the same temperature the solution was cooled to $0{ }^{\circ} \mathrm{C}$. A solution of $\mathrm{NaBH}_{4}(227 \mathrm{mg}, 6.00 \mathrm{mmol}, 2.00 \mathrm{eq})$ in THF:MeOH (10.0 mL, 4:1 v:v, $\left.0.3 \mathrm{M}\right)$ was added via syringe within five minutes and stirring was continued at $0{ }^{\circ} \mathrm{C}$ for another two and a half hours. Saturated $\mathrm{NH}_{4} \mathrm{Cl}$ solution $(10 \mathrm{~mL})$, water $(20 \mathrm{~mL})$ and $\mathrm{CH}_{2} \mathrm{Cl}_{2}(20 \mathrm{~mL})$ were added and the phases separated. The aqueous layer was extracted with $\mathrm{CH}_{2} \mathrm{Cl}_{2}(4 \times 20 \mathrm{~mL})$ and the combined organic phases were dried over anhydrous $\mathrm{Na}_{2} \mathrm{SO}_{4}$. After filtration and solvent evaporation under reduced pressure, the residue was redissolved in THF: $\mathrm{H}_{2} \mathrm{O}(10.0 \mathrm{~mL}, 4: 1 \mathrm{v}: \mathrm{v}, 0.3 \mathrm{M})$. Oxalic acid dihydrate $(756 \mathrm{mg}$, $6.00 \mathrm{mmol}, 2.00 \mathrm{eq}$ ) was added at $25^{\circ} \mathrm{C}$ and the mixture was stirred for 16 hours at the same 
temperature. Saturated $\mathrm{NaHCO}_{3}$ solution $(10 \mathrm{~mL})$, water $(20 \mathrm{~mL})$ and $\mathrm{CH}_{2} \mathrm{Cl}_{2}(40 \mathrm{~mL})$ were added and the phases separated. The aqueous layer was extracted with $\mathrm{CH}_{2} \mathrm{Cl}_{2}(4 \times 20 \mathrm{~mL})$ and the combined organic phases were dried over anhydrous $\mathrm{Na}_{2} \mathrm{SO}_{4}$, filtered and the solvent was evaporated under reduced pressure. Purification by flash column chromatography $\left(\mathrm{SiO}_{2}, \mathrm{CH}: \mathrm{EE}\right.$ 5:1 v:v) afforded 4 (614 mg, $2.56 \mathrm{mmol}, 85 \%$ ) as a colorless oil which solidified very slowly upon standing at $25^{\circ} \mathrm{C}$.

$\mathbf{R}_{\boldsymbol{f}}=0.54\left(\mathrm{SiO}_{2}, \mathrm{CH}: \mathrm{EE}\right.$ 5:1 v:v). Mp.: $26-28{ }^{\circ} \mathrm{C} .{ }^{1} \mathbf{H}-\mathrm{NMR}\left(\mathbf{3 6 0} \mathbf{M H z}, \mathbf{C D C l}_{3}\right): \delta=10.34\left(\mathrm{~d},{ }^{4} \boldsymbol{J}_{\mathrm{HH}}=\right.$ $0.8 \mathrm{~Hz}, 1 \mathrm{H}, \mathrm{CHO}), 7.92\left(\mathrm{~d},{ }^{3} J_{\mathrm{HH}}=8.0 \mathrm{~Hz}, 1 \mathrm{H}, \mathrm{H}-6\right), 7.63\left(\mathrm{dd},{ }^{4} J_{\mathrm{HH}}=1.6 \mathrm{~Hz},{ }^{5} J_{\mathrm{HH}}=0.8 \mathrm{~Hz}, 1 \mathrm{H}, \mathrm{H}-3\right)$, $\left.7.38\left(\mathrm{ddd},{ }^{3} J_{\mathrm{HH}}=7.9 \mathrm{~Hz},{ }^{4} J_{\mathrm{HH}}=1.6 \mathrm{~Hz},{ }^{5} J_{\mathrm{HH}}=0.8 \mathrm{~Hz}, 1 \mathrm{H}, \mathrm{H}-5\right), 4.43(\mathrm{~s}, 2 \mathrm{H}, \mathrm{CH})_{2}\right) \mathrm{ppm} .{ }^{13} \mathbf{C}\left\{{ }^{1} \mathbf{H}\right\}-\mathbf{N M R}$ (91 MHz, $\left.\mathbf{C D C l}_{3}\right): \delta=191.0(\mathrm{C}-7), 143.2$ (C-4), 132.9 (C-1), 132.7 (C-3), 130.0 (C-6), 127.2 (C-2), 126.9 (C-5), 53.3 (C-8) ppm. IR (ATR, neat): $\tilde{v}=3084$ (w), 2920 (w), 2859 (w), 2758 (w), 2227 (w), 2116 (s), 1684 (s), 1599 (s), 1558 (m), 1483 (w), 1427 (m), 1403 (m), 1387 (m), 1339 (s), 1275 (s), 1207 (s), 1140 (m), 1038 (m), 966 (m), 957 (m), 881 (m), 872 (m), 821 (s), 797 (s), 733 (w), 681 (m) cm-1. MS (EI, 70 eV): $\mathrm{m} / \mathrm{z}=238.8\left[\mathrm{C}_{8} \mathrm{H}_{6}{ }^{79} \mathrm{BrN}_{3} \mathrm{O}\right]^{+\cdot}$. HR-MS (EI, 70 eV): calculated for $\mathrm{C}_{8} \mathrm{H}_{6}{ }^{79} \mathrm{BrN}_{3} \mathrm{O}^{+\cdot}$ $[\mathrm{M}]^{+}: \mathrm{m} / \mathrm{z}=238.96888$, found: 238.96872 (Dev.: $-0.16 \mathrm{mu} ;-0.65 \mathrm{ppm}$ ). 


\subsection{Preparation of Highly Functionalized Fluorenes (5), (23), (25) \& (28)}
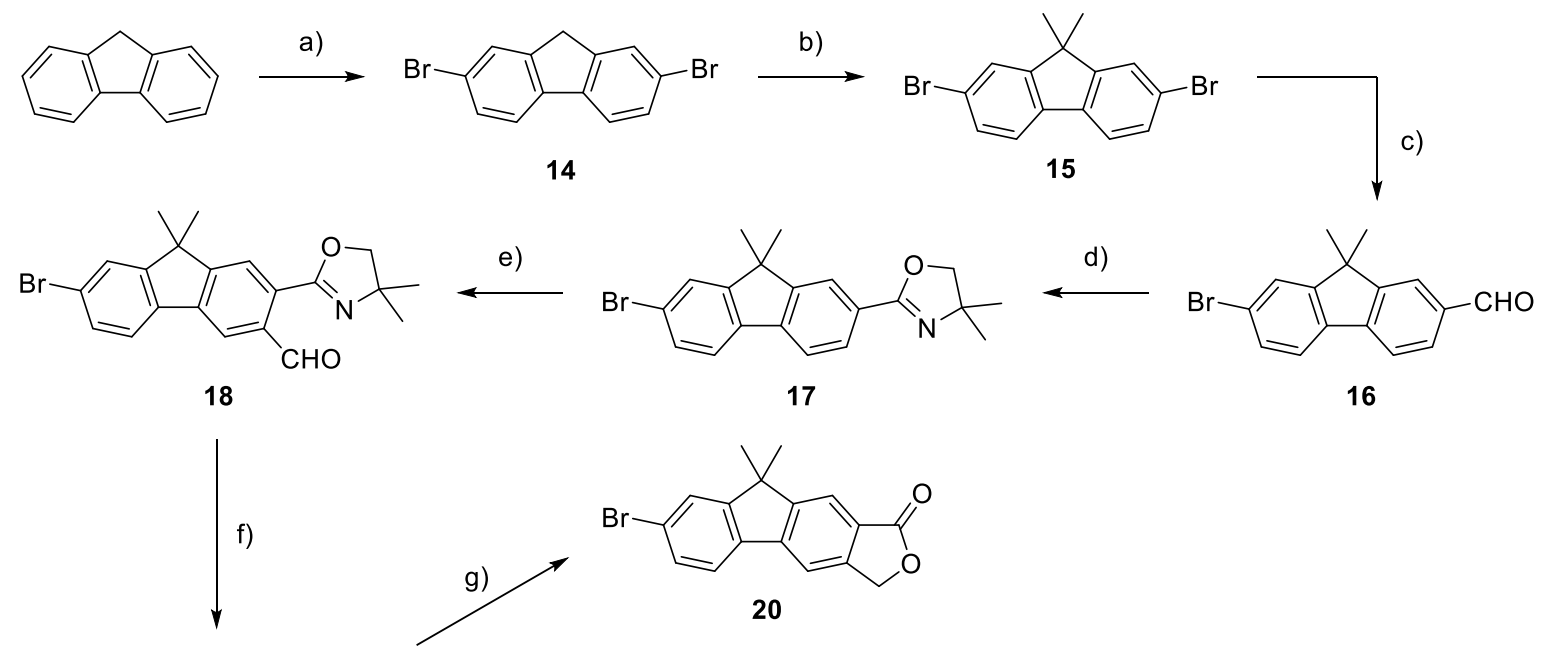

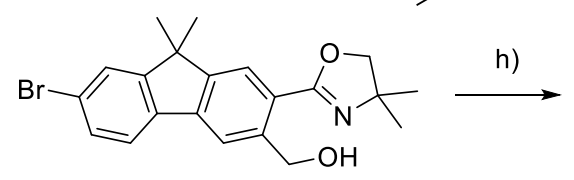

19
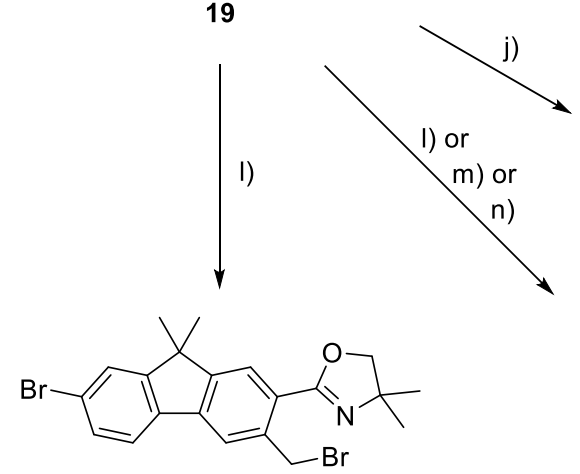

24

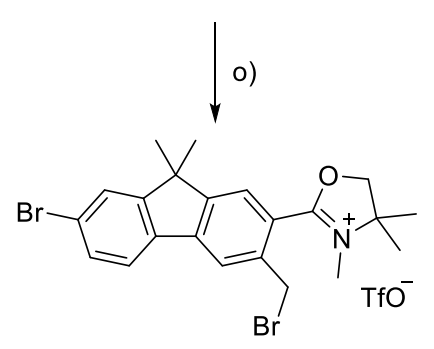

26

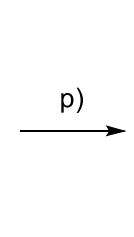

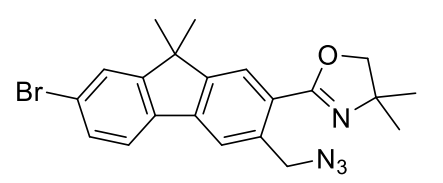

21

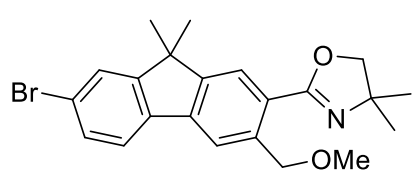

22

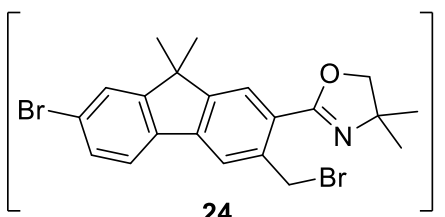

24

(n)

27
16

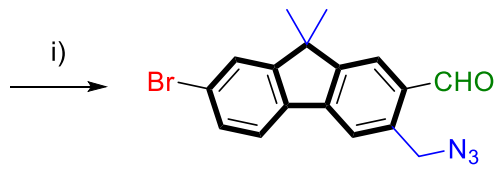

5
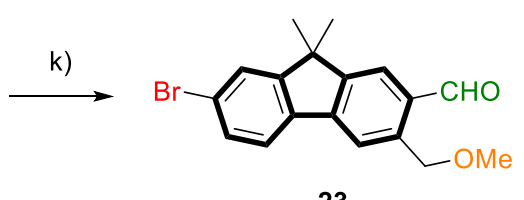

23
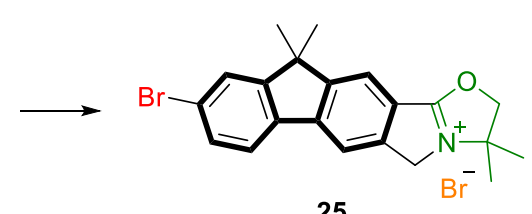

25

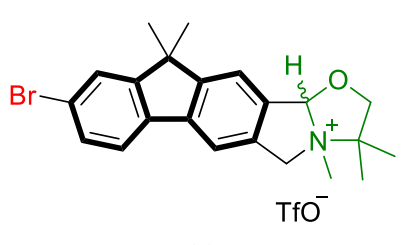

28

Reaction conditions: a) $\mathrm{Br}_{2}, \mathrm{Fe}$-powder, $\mathrm{CHCl}_{3}, 0{ }^{\circ} \mathrm{C}, 4 \mathrm{~h}, 99 \%$; b) KOH, KI, MeI, DMSO, $25{ }^{\circ} \mathrm{C}, 18 \mathrm{~h}, 92 \%$; c) 1$) n \mathrm{BuLi}$, THF, $-78{ }^{\circ} \mathrm{C}, 1 \mathrm{~h}$; 2) DMF, THF, $-78{ }^{\circ} \mathrm{C}$ to $25^{\circ} \mathrm{C}, 10 \mathrm{~h}, 90 \%$; d) 1) 2-amino-2-methylpropan-1-ol, $4 \AA \mathrm{MS}, \mathrm{CH}_{2} \mathrm{Cl}_{2}, 25^{\circ} \mathrm{C}, 18 \mathrm{~h}$; 2) NBS, $\mathrm{CH}_{2} \mathrm{Cl}_{2}, 25^{\circ} \mathrm{C}, 3 \mathrm{~h}, 92 \%$; e) 1) TMPMgCl$\cdot \mathrm{LiCl}, \mathrm{THF}, 25^{\circ} \mathrm{C}, 4 \mathrm{~h}$; 2) DMF, THF, $-15{ }^{\circ} \mathrm{C}$ to $25{ }^{\circ} \mathrm{C}, 2 \mathrm{~h}$; f) $\mathrm{NaBH}_{4}$, THF:MeOH 1:1 v:v, $0{ }^{\circ} \mathrm{C}, 1.5 \mathrm{~h}, 79 \%$ (2 steps); g) $\mathrm{HCl}(4 \mathrm{~N}), 120{ }^{\circ} \mathrm{C}, 6 \mathrm{~h}$, $91 \%$; h) 1) DPPA, DBU, PhMe, $25{ }^{\circ} \mathrm{C}, 15 \mathrm{~h}$; 2) $\mathrm{NaN}_{3}, \mathrm{PhMe}, 60{ }^{\circ} \mathrm{C}, 4 \mathrm{~h}, 87 \%$; i) 1) $\mathrm{MeOTf}, \mathrm{CH}_{2} \mathrm{Cl}_{2}, 25{ }^{\circ} \mathrm{C}$, $2.5 \mathrm{~h} ; 2) \mathrm{NaBH}_{4}$, THF:MeOH 4:1 v:v, $0{ }^{\circ} \mathrm{C}, 2.5 \mathrm{~h}$; 3) oxalic acid, THF: $\mathrm{H}_{2} \mathrm{O} 4: 1 \mathrm{v}: \mathrm{v}, 2{ }^{\circ} \mathrm{C}, 20 \mathrm{~h}, 86 \%$ - overall yield to $5: 45 \%$ (8 steps); j) 1) NaH, THF, $0{ }^{\circ} \mathrm{C}, 30 \mathrm{~min}$; 2) MeI, THF, $0{ }^{\circ} \mathrm{C}$ to $25{ }^{\circ} \mathrm{C}, 2 \mathrm{~h}, 99 \%$; k) 1) MeOTf, $\left.\mathrm{CH}_{2} \mathrm{Cl}_{2}, 25^{\circ} \mathrm{C}, 3 \mathrm{~h} ; 2\right) \mathrm{NaBH}_{4}$, THF:MeOH 4:1 v:v, $0^{\circ} \mathrm{C}, 3 \mathrm{~h}$; 3 ) oxalic acid, THF: $\mathrm{H}_{2} \mathrm{O}$ 4:1 v:v, $25{ }^{\circ} \mathrm{C}, 24 \mathrm{~h}, 75 \%$ - overall yield to 23: $44 \%$ (8 steps); l) $\mathrm{CBr}_{4}, \mathrm{PPh}_{3}, \mathrm{CH}_{2} \mathrm{Cl}_{2}, 0{ }^{\circ} \mathrm{C}$ to $25^{\circ} \mathrm{C}, 2 \mathrm{~h}, 95 \%$; m) NBS, $\mathrm{PPh}_{3}, \mathrm{CH}_{2} \mathrm{Cl}_{2}, 0{ }^{\circ} \mathrm{C}$ 
to $25^{\circ} \mathrm{C}, 2 \mathrm{~h}, 91 \%$; n) $\mathrm{PBr}_{3}, \mathrm{CH}_{2} \mathrm{Cl}_{2}, 0{ }^{\circ} \mathrm{C}$ to $25^{\circ} \mathrm{C}, 2 \mathrm{~h}, 92 \%$; o) $\left.\mathrm{MeOTf} \mathrm{CH}_{2} \mathrm{Cl}_{2}, 25^{\circ} \mathrm{C}, 3 \mathrm{~h} ; \mathrm{p}\right) \mathrm{NaBH}$, THF:MeOH 4:1 v:v, $0{ }^{\circ} \mathrm{C}, 3$ h, $56 \%$ from 19.

\subsubsection{2,7-Dibromo-9H-fluorene (14)}

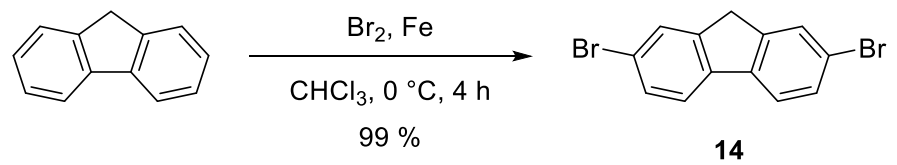

A modified literature procedure was used.[18] Fluorene (8.31 g, $50.0 \mathrm{mmol}, 1.00 \mathrm{eq})$ was dissolved in $\mathrm{CHCl}_{3}(83 \mathrm{~mL}, 0.6 \mathrm{M})$ and Fe-powder $(279 \mathrm{mg}, 5.00 \mathrm{mmol}, 0.10 \mathrm{eq})$ was added. The solution was cooled to $0{ }^{\circ} \mathrm{C}$ in a water/ice bath. $\mathrm{Br}_{2}(5.38 \mathrm{~mL}, 105 \mathrm{mmol}, 2.10 \mathrm{eq})$ in $\mathrm{CHCl}_{3}(42 \mathrm{~mL})$ was added through a dropping funnel over one hour in the dark to the vigorously stirred mixture. After complete addition the mixture was stirred for an additional three hours at $0{ }^{\circ} \mathrm{C}$. Saturated $\mathrm{Na}_{2} \mathrm{~S}_{2} \mathrm{O}_{5}$ solution (100 $\mathrm{mL})$ was slowly added at the same temperature and stirring was continued for 30 minutes. $\mathrm{CHCl}_{3}(100$ $\mathrm{mL})$ was added, the phases were separated and the aqueous layer was extracted with $\mathrm{CHCl}_{3}(2 \times 100$ $\mathrm{mL})$. The combined organic layers were dried over anhydrous $\mathrm{Na}_{2} \mathrm{SO}_{4}$, filtered and concentrated under reduced pressure on a rotary evaporator. $14(16.1 \mathrm{~g}, 49.7 \mathrm{mmol}, 99 \%)$ was isolated as a colorless solid. If desired the product can be recrystallized from chloroform.

$\mathbf{R}_{f}=0.61\left(\mathrm{SiO}_{2}, \mathrm{CH}\right) . \mathbf{M p} .: 164-166{ }^{\circ} \mathrm{C} .{ }^{\mathbf{1}} \mathbf{H}-\mathbf{N M R}\left(\mathbf{3 6 0} \mathbf{M H z}, \mathbf{C D C l}_{3}\right): \delta=7.64\left(\mathrm{~d},{ }^{4} J_{\mathrm{HH}}=1.6 \mathrm{~Hz}\right.$, 2H), $7.57\left(\mathrm{~d},{ }^{3} J_{\mathrm{HH}}=8.1 \mathrm{~Hz}, 2 \mathrm{H}\right), 7.49\left(\mathrm{dd},{ }^{3} J_{\mathrm{HH}}=8.2 \mathrm{~Hz},{ }^{4} J_{\mathrm{HH}}=1.8 \mathrm{~Hz}, 2 \mathrm{H}\right), 3.83(\mathrm{~s}, 2 \mathrm{H}) \mathrm{ppm} .{ }^{13} \mathbf{C}\left\{{ }^{1} \mathbf{H}\right\}-$ NMR (91 MHz, CDCl 3 ): $\delta=144.9$ (2x), 139.8 (2x), $130.2(2 \mathrm{x}), 128.4(2 \mathrm{x}), 121.3(2 \mathrm{x}), 121.1(2 \mathrm{x}), 36.7$ ppm. IR (ATR, neat): $\tilde{v}=3049$ (w), 2919 (w), 1881 (w), 1760 (w), 1568 (w), 1453 (w), 1391 (m), 1159 (w), 1054 (m), 1005 (w), 952 (w), 931 (w), 807 (s), 685 (m), $662(\mathrm{~m}) \mathrm{cm}^{-1}$. MS (EI, 70 eV): m/z = 321.8 $\left[\mathrm{C}_{13} \mathrm{H}_{8}{ }^{79} \mathrm{Br}_{2}\right]^{+\cdot}$. The analytical data are in accordance with the literature.[19]

\subsubsection{2,7-Dibromo-9,9-dimethyl-9H-fluorene (15)}

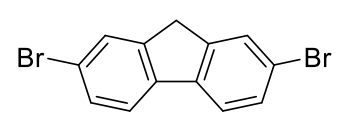

14

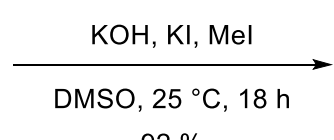

$92 \%$

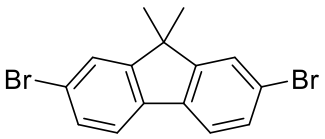

15

A modified literature procedure was used.[20] 14 (16.2 g, $50.0 \mathrm{mmol}, 1.00 \mathrm{eq})$ was suspended in DMSO $(83 \mathrm{~mL}, 0.6 \mathrm{M})$ and $\mathrm{KI}(830 \mathrm{mg}, 5.00 \mathrm{mmol}, 0.10 \mathrm{eq})$ was added. To the water bath-cooled and vigorously stirred mixture were added $\mathrm{KOH}$ pellets $(11.2 \mathrm{~g}, 200 \mathrm{mmol}, 4.00 \mathrm{eq})$. The reaction was stirred for one hour, while the solution turned intensive red. MeI (7.78 mL, $125 \mathrm{mmol}, 2.50 \mathrm{eq})$ was added via syringe pump $(0.15 \mathrm{~mL} / \mathrm{min})$ through a rubber septum and stirring was continued at $25{ }^{\circ} \mathrm{C}$ for 18 hours. Excess of MeI was quenched by addition of $\mathrm{NEt}_{3}(13.9 \mathrm{~mL}, 100 \mathrm{mmol}, 2.00 \mathrm{eq})$. The mixture was stirred 
for 30 minutes, poured into water $(500 \mathrm{~mL})$ and extracted with $\mathrm{CH}_{2} \mathrm{Cl}_{2}(4 \times 100 \mathrm{~mL})$. The combined organic layers were dried over anhydrous $\mathrm{NaSO}_{4}$, filtered and concentrated under reduced pressure on a rotary evaporator. Purification by flash column chromatography $\left(\mathrm{SiO}_{2}, \mathrm{CH}\right)$ afforded $15(16.2 \mathrm{~g}$, $46.0 \mathrm{mmol}, 92 \%$ ) as a colorless solid. If desired the product can be recrystallized from cyclohexane.

$\mathbf{R}_{f}=0.68\left(\mathrm{SiO}_{2}, \mathrm{CH}\right)$. Mp.: $177-179{ }^{\circ} \mathrm{C} .{ }^{1} \mathbf{H}-\mathbf{N M R}\left(\mathbf{3 6 0} \mathbf{M H z}, \mathbf{C D C l}_{3}\right): \delta=7.55\left(\mathrm{~d},{ }^{4} J_{\mathrm{HH}}=1.8 \mathrm{~Hz}\right.$, $2 \mathrm{H}), 7.54\left(\mathrm{~d},{ }^{3} J_{\mathrm{HH}}=8.1 \mathrm{~Hz}, 2 \mathrm{H}\right), 7.46\left(\mathrm{dd},{ }^{3} J_{\mathrm{HH}}=8.1 \mathrm{~Hz},{ }^{4} J_{\mathrm{HH}}=1.8 \mathrm{~Hz}, 2 \mathrm{H}\right), 1.47(\mathrm{~s}, 6 \mathrm{H}) \mathrm{ppm} .{ }^{13} \mathbf{C}\left\{{ }^{1} \mathbf{H}\right\}-$ NMR (91 MHz, CDCl $\left.\mathbf{C l}_{3}\right): \delta=155.4$ (2x), 137.3 (2x), 130.5 (2x), 126.3 (2x), $121.6(4 \mathrm{x}), 47.4,27.0(2 \mathrm{x})$ ppm. IR (ATR, neat): $\tilde{v}=2962$ (m), 2920 (w), 2856 (w), 1861 (w), 1727 (w), 1597 (w), 1577 (w), 1447 (m), 1397 (m), 1259 (m), 1083 (m), 1058 (m), 1001 (m), 865 (m), 824 (m), 791 (s), 729 (m), 667 (m) $\mathrm{cm}^{-1}$. MS $(\mathbf{E I}, 70 \mathrm{eV}): \mathrm{m} / \mathrm{z}=349.9\left[\mathrm{C}_{15} \mathrm{H}_{12}{ }^{79} \mathrm{Br}_{2}\right]^{+.}$. The analytical data are in accordance with the literature.[21]

\subsubsection{7-Bromo-9,9-dimethyl-9H-fluorene-2-carbaldehyde (16)}

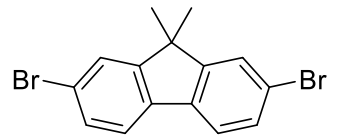

15

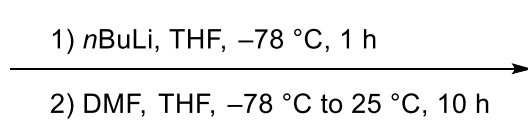

$90 \%$

A modified literature procedure was used.[20] A heat gun-dried and nitrogen-flushed Schlenk flask was charged with 15 (14.1 g, $40.0 \mathrm{mmol}, 1.00 \mathrm{eq})$ and anhydrous THF (200 mL, $0.2 \mathrm{M})$ was added. The solution was cooled to $-78^{\circ} \mathrm{C}$ and $n \mathrm{BuLi}(16.8 \mathrm{~mL}, 42.0 \mathrm{mmol}, 1.05 \mathrm{eq}, 2.5 \mathrm{M}$ in hexane) was added via syringe pump $(0.2 \mathrm{~mL} / \mathrm{min})$ through a rubber septum to the vigorously stirred mixture. The intensive red solution was stirred for one hour at $-78{ }^{\circ} \mathrm{C}$ and anhydrous DMF $(5.85 \mathrm{~g}, 6.19 \mathrm{~mL}, 80.0 \mathrm{mmol}$, $2.00 \mathrm{eq})$ in anhydrous THF $(8 \mathrm{~mL})$ was added via syringe pump $(0.4 \mathrm{~mL} / \mathrm{min})$ through a rubber septum. Stirring was continued for 10 hours while the mixture was allowed to warm slowly to $25{ }^{\circ} \mathrm{C}$. $\mathrm{HCl}$ $(100 \mathrm{~mL}, 1.0 \mathrm{M})$ was poured into the reaction in small portions and the mixture was stirred rapidly for 30 minutes. The phases were separated and the aqueous layer was extracted with ethyl acetate $(3 \times 100 \mathrm{~mL})$. The combined organic layers were dried over anhydrous $\mathrm{Na}_{2} \mathrm{SO}_{4}$, filtered and concentrated under reduced pressure on a rotary evaporator. Purification by flash column chromatography ( $\mathrm{SiO}_{2}$, CH:EE 20:1 v:v) afforded $\mathbf{1 6}(10.9 \mathrm{~g}, 36.0 \mathrm{mmol}, 90 \%)$ as a colorless solid.

$\mathbf{R}_{f}=0.26\left(\mathrm{SiO}_{2}, \mathrm{CH}: \mathrm{EE} 20: 1 \mathrm{v}: \mathrm{v}\right) . \mathbf{M p} .: 147-149^{\circ} \mathrm{C} .{ }^{1} \mathbf{H}-\mathbf{N M R}\left(\mathbf{3 6 0} \mathbf{~ M H z}, \mathbf{C D C l}_{3}\right): \delta=10.06(\mathrm{~s}, 1 \mathrm{H})$, $7.96\left(\mathrm{~d},{ }^{4} J_{\mathrm{HH}}=1.5 \mathrm{~Hz}, 1 \mathrm{H}\right), 7.87\left(\mathrm{dd},{ }^{3} J_{\mathrm{HH}}=7.8 \mathrm{~Hz},{ }^{4} J_{\mathrm{HH}}=1.3 \mathrm{~Hz}, 1 \mathrm{H}\right), 7.82\left(\mathrm{~d},{ }^{3} J_{\mathrm{HH}}=7.8 \mathrm{~Hz}, 1 \mathrm{H}\right)$, $7.65\left(\mathrm{~d},{ }^{3} J_{\mathrm{HH}}=8.1 \mathrm{~Hz}, 1 \mathrm{H}\right), 7.61\left(\mathrm{~d},{ }^{4} J_{\mathrm{HH}}=1.6 \mathrm{~Hz}, 1 \mathrm{H}\right), 7.51\left(\mathrm{dd},{ }^{3} J_{\mathrm{HH}}=8.1 \mathrm{~Hz},{ }^{4} J_{\mathrm{HH}}=1.7 \mathrm{~Hz}, 1 \mathrm{H}\right)$, 1.52 (s, 6H) ppm. ${ }^{13} \mathbf{C}\left\{{ }^{1} \mathbf{H}\right\}-N M R\left(\mathbf{9 1} \mathbf{M H z}, \mathbf{C D C l}_{3}\right): \delta=192.2,157.0,154.1,144.6,136.8,135.9,130.8$, 130.7, 126.6, 123.2, 122.7, 120.5, 47.4, 26.9 (2x) ppm. IR (ATR, neat): $\tilde{v}=2963$ (w), 2924 (w), 2814 (w), $2783(\mathrm{w}), 2708(\mathrm{w}), 1695(\mathrm{~s}), 1683(\mathrm{~s}), 1605$ (m), $1405(\mathrm{~m}), 1247$ (m), $1175(\mathrm{~s}), 1060(\mathrm{~m}), 883(\mathrm{~m})$, 
$810(\mathrm{~s}), 795$ (s), $755(\mathrm{~s}), 733(\mathrm{~s}), 658(\mathrm{~m}) \mathrm{cm}^{-1}$. MS (APCI): m/z = $301.1\left[\mathrm{C}_{16} \mathrm{H}_{13}{ }^{79} \mathrm{BrO}+\mathrm{H}\right]^{+}$. The analytical data are in accordance with the literature.[22]

\subsubsection{2-(7-Bromo-9,9-dimethyl-9H-fluoren-2-yl)-4,4-dimethyl-4,5-dihydrooxazole (17)}

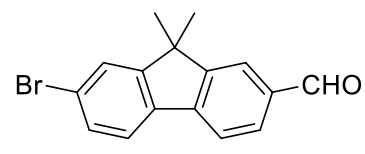

16

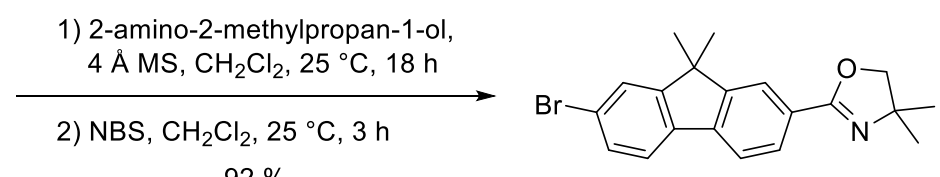

$92 \%$

17

A modified literature procedure was used.[8] $16(3.01 \mathrm{~g}, 10.0 \mathrm{mmol}, 1.00 \mathrm{eq})$ and 2-amino-2methylpropan-1-ol (1.78 g, $1.91 \mathrm{~mL}, 20.0 \mathrm{mmol}, 2.00 \mathrm{eq})$ were dissolved in $\mathrm{CH}_{2} \mathrm{Cl}_{2}(40 \mathrm{~mL}, 0.25 \mathrm{M})$ and $4 \AA$ MS $(5.00 \mathrm{~g})$ was added. The mixture was slowly stirred for 18 hours at $25^{\circ} \mathrm{C}$ whereupon NBS ( $3.56 \mathrm{~g}, 20.0 \mathrm{mmol}, 2.00 \mathrm{eq}$ ) was added in one portion and stirring was continued for another three hours at the same temperature. All solids were filtered off and the organic phase was washed with saturated $\mathrm{NaHCO}_{3}$ solution $(2 \times 50 \mathrm{~mL})$. The combined aqueous phases were extracted with $\mathrm{CH}_{2} \mathrm{Cl}_{2}(2 \times 50 \mathrm{~mL})$. All organic phases were combined and washed with saturated $\mathrm{Na}_{2} \mathrm{~S}_{2} \mathrm{O}_{3}$ solution $(50 \mathrm{~mL})$ and the aqueous phase was extracted with $\mathrm{CH}_{2} \mathrm{Cl}_{2}(20 \mathrm{~mL})$. The combined organic phases were dried over anhydrous $\mathrm{MgSO}_{4}$, filtered and concentrated under reduced pressure on a rotary evaporator. Purification by flash column chromatography ( $\mathrm{SiO}_{2}$, $\left.\mathrm{CH}: \mathrm{EE} 15: 1 \mathrm{v}: \mathrm{v}\right)$ afforded $\mathbf{1 7}(3.41 \mathrm{~g}, 9.21 \mathrm{mmol}, 92 \%)$ as a colorless foam.

$\mathbf{R}_{f}=0.17\left(\mathrm{SiO}_{2}, \mathrm{CH}: \mathrm{EE}\right.$ 15:1 v:v). Mp.: $62-64{ }^{\circ} \mathrm{C} .{ }^{1} \mathbf{H}-\mathrm{NMR}\left(\mathbf{3 6 0} \mathbf{M H z}, \mathbf{C D C l}_{3}\right): \delta=8.02\left(\mathrm{~d},{ }^{4} \boldsymbol{J}_{\mathrm{HH}}=\right.$ $1.4 \mathrm{~Hz}, 1 \mathrm{H}), 7.92\left(\mathrm{dd},{ }^{3} J_{\mathrm{HH}}=7.9 \mathrm{~Hz},{ }^{4} J_{\mathrm{HH}}=1.5 \mathrm{~Hz}, 1 \mathrm{H}\right), 7.69\left(\mathrm{~d},{ }^{3} J_{\mathrm{HH}}=7.9 \mathrm{~Hz}, 1 \mathrm{H}\right), 7.58\left(\mathrm{~d},{ }^{3} J_{\mathrm{HH}}=8.0\right.$ $\mathrm{Hz}, 1 \mathrm{H}), 7.56\left(\mathrm{~d},{ }^{4} J_{\mathrm{HH}}=1.8 \mathrm{~Hz}, 1 \mathrm{H}\right), 7.46\left(\mathrm{dd},{ }^{3} J_{\mathrm{HH}}=8.0 \mathrm{~Hz},{ }^{4} J_{\mathrm{HH}}=1.9 \mathrm{~Hz}, 1 \mathrm{H}\right), 4.12(\mathrm{~s}, 2 \mathrm{H}), 1.48(\mathrm{~s}$, 6H), 1.41 (s, 6H) ppm. ${ }^{13} \mathbf{C}\left\{{ }^{1} \mathbf{H}\right\}-N M R\left(\mathbf{9 1} \mathbf{~ M H z}, \mathbf{C D C l}_{3}\right): \delta=162.3,156.4,153.3,141.2,137.4,130.4$, 127.8, 127.2, 126.4, 122.7, 122.1, 122.0, 119.9, 79.2, 67.7, 47.4, 28.6 (2x), 26.9 (2x) ppm. IR (ATR, neat): $\tilde{v}=2962(\mathrm{w}), 2924(\mathrm{w}), 2359$ (w), 1641 (s), 1452 (m), 1403 (m), 1355 (m), 1308 (s), 1262 (s), 1202 (s), 1086 (m), 1060 (s), 968 (m), 815 (s), 774 (m), 738 (s), $716(\mathrm{~s}) \mathrm{cm}^{-1}$. MS (APCI): m/z = 370.2 $\left[\mathrm{C}_{20} \mathrm{H}_{20}{ }^{79} \mathrm{BrNO}+\mathrm{H}\right]^{+}$. HR-MS (EI, $70 \mathrm{eV}$ ): calculated for $\mathrm{C}_{20} \mathrm{H}_{20}{ }^{79} \mathrm{BrNO}^{+\cdot}[\mathrm{M}]^{+*}: \mathrm{m} / \mathrm{z}=369.07228$, found: 369.07227 (Dev.: $-0.01 \mathrm{mu} ;-0.02 \mathrm{ppm}$ ). The analytical data are in accordance with the literature.[23]

\subsubsection{7-Bromo-2-(4,4-dimethyl-4,5-dihydrooxazol-2-yl)-9,9-dimethyl-9H-fluorene-3- carbaldehyde (18)}

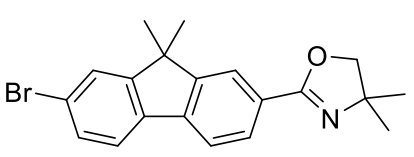

17
1) $\mathrm{TMPMgCl} \cdot \mathrm{LiCl}, \mathrm{THF}, 25^{\circ} \mathrm{C}, 4 \mathrm{~h}$

2) DMF, THF, $-15^{\circ} \mathrm{C}$ to $25^{\circ} \mathrm{C}, 2 \mathrm{~h}$

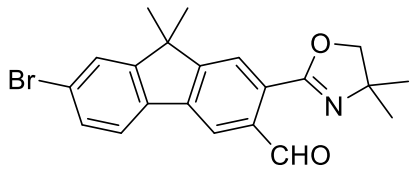

18 
A literature procedure was used.[23] A heat gun-dried and nitrogen-flushed Schlenk flask, equipped with a magnetic stirring bar and a rubber septum, was charged with 17 (3.70 g, $10.0 \mathrm{mmol}, 1.00 \mathrm{eq})$ and evacuated for half an hour. The flask was flushed with nitrogen and anhydrous THF (25.0 mL, $0.4 \mathrm{M})$ was added. Addition of TMPMgCl$\cdot \mathrm{LiCl}(25.0 \mathrm{~mL}, 30.0 \mathrm{mmol}, 3.00 \mathrm{eq}, 1.20 \mathrm{M})$ via syringe through the rubber septum within five minutes at $25{ }^{\circ} \mathrm{C}$ was followed by stirring for four hours under the same conditions. DMF (3.10 mL, $40.0 \mathrm{mmol}, 4.00 \mathrm{eq})$ was diluted in anhydrous THF (10 mL) and added via syringe pump $(0.3 \mathrm{~mL} / \mathrm{min})$ through the rubber septum to the dark red solution at $-15^{\circ} \mathrm{C}$. After complete addition the brown mixture was stirred for another two hours while it was allowed to warm to $25^{\circ} \mathrm{C}$. Full consumption of the starting material was followed by diluting with water $(50 \mathrm{~mL})$ and ethyl acetate $(80 \mathrm{~mL})$. The phases were separated and the aqueous layer was extracted with ethyl acetate $(4 \times 80 \mathrm{~mL})$. The combined organic layers were dried over anhydrous $\mathrm{Na}_{2} \mathrm{SO}_{4}$, filtered and concentrated under reduced pressure on a rotary evaporator $\left(30^{\circ} \mathrm{C}\right.$ water bath temperature). The product was used without further purification for the next reaction step. If desired the aldehyde $\mathbf{1 8}$ can be isolated as a colorless solid by washing the crude product with small amounts of ice cooled cyclohexane.

$\mathbf{R}_{\boldsymbol{f}}=0.33\left(\mathrm{SiO}_{2}\right.$, CH:EE 6:1 v:v). Mp.: $178-180{ }^{\circ} \mathrm{C}$ decomp. ${ }^{1} \mathbf{H}-\mathbf{N M R}\left(\mathbf{3 6 0} \mathbf{M H z}, \mathbf{C D C l}_{3}\right): \delta=10.75$ $(\mathrm{s}, 1 \mathrm{H}), 8.26(\mathrm{~s}, 1 \mathrm{H}), 7.93(\mathrm{~s}, 1 \mathrm{H}), 7.67\left(\mathrm{~d},{ }^{3} J_{\mathrm{HH}}=8.1 \mathrm{~Hz}, 1 \mathrm{H}\right), 7.58(\mathrm{~s}, 1 \mathrm{H}), 7.51\left(\mathrm{~d},{ }^{3} J_{\mathrm{HH}}=8.0 \mathrm{~Hz}, 1 \mathrm{H}\right)$, 4.19 (s, 2H), 1.51 (s, 6H), 1.44 (s, 6H) ppm. ${ }^{13} \mathbf{C}\left\{{ }^{1} \mathbf{H}\right\}-N M R$ (91 MHz, $\left.\mathbf{C D C l}_{3}\right): \delta=192.5,160.7,158.1$, 156.1, 141.2, 136.5, 136.1, 130.8, 129.5, 126.5, 124.4, 122.9, 122.6, 119.7, 79.5, 68.9, 47.8, $28.6(2 \mathrm{x})$, 26.8 (2x) ppm. IR (ATR, neat): $\tilde{v}=2966$ (w), 2926 (w), 2359 (w), 2324 (w), $1682(\mathrm{~s}), 1643$ (m), 1613 (m), 1463 (m), 1409 (m), 1358 (m), 1302 (m), 1268 (m), 1200 (m), 1146 (m), 1086 (m), 1062 (s), 1038 (s), 973 (s), 899 (s), 810 (s), $743(\mathrm{~s}) \mathrm{cm}^{-1}$. MS (APCI): m/z = $398.1\left[\mathrm{C}_{21} \mathrm{H}_{20}{ }^{79} \mathrm{BrNO}_{2}+\mathrm{H}\right]^{+}$. HR-MS (EI, $70 \mathrm{eV}$ ): calculated for $\mathrm{C}_{21} \mathrm{H}_{20}{ }^{79} \mathrm{BrNO}_{2}{ }^{+\cdot}[\mathrm{M}]^{+*}: \mathrm{m} / \mathrm{z}=397.06719$, found: 397.06645 (Dev.: $-0.74 \mathrm{mu}$; $-1.87 \mathrm{ppm})$. The analytical data are in accordance with the literature.[23]

\subsection{6 (7-Bromo-2-(4,4-dimethyl-4,5-dihydrooxazol-2-yl)-9,9-dimethyl-9H-fluoren-3- yl)methanol (19)}

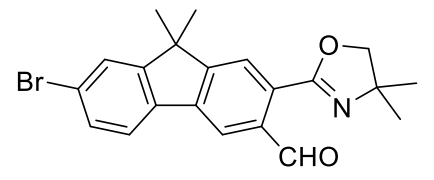

18

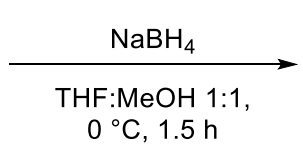

$79 \%$ (two steps)

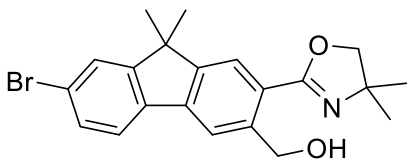

19

A modified literature procedure was used.[10] The crude aldehyde 18 (10.0 mmol, 1.00 eq) was dissolved in THF:MeOH $(25.0 \mathrm{~mL}, 1: 1 \mathrm{v}: \mathrm{v}, 0.4 \mathrm{M})$ and cooled to $0{ }^{\circ} \mathrm{C} . \mathrm{NaBH}_{4}(757 \mathrm{mg}, 20.0 \mathrm{mmol}$, $2.00 \mathrm{eq})$ was added at once and stirring was continued for 90 minutes while the temperature was maintained. Water $(25 \mathrm{~mL})$ was added dropwise at $0{ }^{\circ} \mathrm{C}$ to stop the reaction, $\mathrm{CH}_{2} \mathrm{Cl}_{2}(50 \mathrm{~mL})$ was added and the phases were separated. The aqueous layer was extracted with $\mathrm{CH}_{2} \mathrm{Cl}_{2}(4 \times 50 \mathrm{~mL})$ and the combined organic layers were dried over anhydrous $\mathrm{Na}_{2} \mathrm{SO}_{4}$, filtered and concentrated under reduced 
pressure. Purification by flash column chromatography $\left(\mathrm{SiO}_{2}, \mathrm{CH}: \mathrm{EE} \mathrm{10:1} \mathrm{to} \mathrm{6:1} \mathrm{v:v)} \mathrm{afforded} 19\right.$ (3.16 g, $7.89 \mathrm{mmol}, 79 \%$ over two steps) as a colorless solid. If necessary, the product can be washed with cyclohexane to remove yellow impurities. $\mathrm{CDCl}_{3}$, used for NMR analysis, must be free of acids, due to decomposition of $\mathbf{1 9}$ in acidic media. Therefore, $\mathrm{CDCl}_{3}$ was flushed over neutral alumina.

$\mathbf{R}_{f}=0.06\left(\mathrm{SiO}_{2}, \mathrm{CH}: \mathrm{EE} \mathrm{10:1} \mathrm{v:v).} \mathbf{R}_{f}=0.22\left(\mathrm{SiO}_{2}, \mathrm{CH}: \mathrm{EE}\right.\right.$ 6:1 v:v). Mp.: $179-181{ }^{\circ} \mathrm{C} .{ }^{1} \mathbf{H}-\mathbf{N M R}$ (360 MHz, CDCl $): \delta=7.91(\mathrm{~s}, 1 \mathrm{H}), 7.68(\mathrm{~s}, 1 \mathrm{H}), 7.61\left(\mathrm{~d},{ }^{3} J_{\mathrm{HH}}=8.0 \mathrm{~Hz}, 1 \mathrm{H}\right), 7.57\left(\mathrm{~d},{ }^{4} J_{\mathrm{HH}}=1.8 \mathrm{~Hz}\right.$, $1 \mathrm{H}), 7.49\left(\mathrm{dd},{ }^{3} J_{\mathrm{HH}}=8.0 \mathrm{~Hz},{ }^{4} J_{\mathrm{HH}}=1.8 \mathrm{~Hz}, 1 \mathrm{H}\right), 6.77$ (br. s, $\left.1 \mathrm{H}\right), 4.74(\mathrm{~s}, 2 \mathrm{H}), 4.17(\mathrm{~s}, 2 \mathrm{H}), 1.48(\mathrm{~s}$, 6H), 1.43 (s, 6H) ppm. ${ }^{13} \mathbf{C}\left\{{ }^{1} \mathbf{H}\right\}-N M R\left(\mathbf{9 1} \mathbf{~ M H z}, \mathbf{C D C l}_{3}\right): \delta=162.4,156.7,152.5,141.9,141.4,137.2$, 130.6, 126.4, 126.0, 124.4, 122.4, 122.2 (2x), 78.9, 68.2, 64.9, 47.3, 28.6 (2x), 26.9 (2x) ppm. IR (ATR, neat): $\tilde{v}=3184$ (br), 3050 (w), 2962 (w), 2947 (w), 2926 (w), 2864 (w), 2360 (w), 1635 (s), 1567 (w), 1459 (m), 1366 (m), 1307 (m), 1298 (m), 1204 (s), 1089 (m), 1064 (s), 1050 (s), 1019 (s), 974 (m), 950 (s), 899 (m), 885 (m), 830 (s), 819 (s), 740 (s), 724 (s), 654 (s) $\mathrm{cm}^{-1}$. MS (APCI): m/z = 400.2 $\left[\mathrm{C}_{21} \mathrm{H}_{22}{ }^{79} \mathrm{BrNO}_{2}+\mathrm{H}\right]^{+}$. HR-MS (EI, $\left.70 \mathrm{eV}\right)$ : calculated for $\mathrm{C}_{21} \mathrm{H}_{22}{ }^{79} \mathrm{BrNO}_{2}{ }^{+\cdot}[\mathrm{M}]^{+*}: \mathrm{m} / \mathrm{z}=399.08284$, found: 399.08234 (Dev.: $-0.50 \mathrm{mu} ;-1.25 \mathrm{ppm}$ ). The analytical data are in accordance with the literature.[23]

\subsubsection{7-Bromo-9,9-dimethyl-3,9-dihydro-1H-fluoreno[2,3-c]furan-1-one (20)}

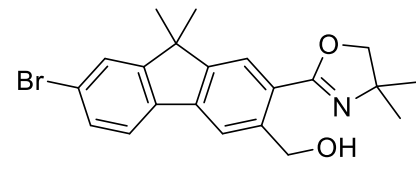

19

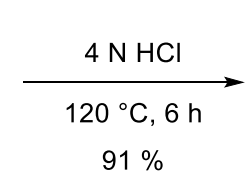

$91 \%$

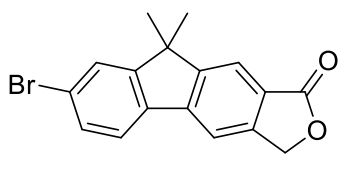

20

A modified literature procedure was used.[24] 19 (160 mg, $400 \mu \mathrm{mol}, 1.00 \mathrm{eq})$ was suspended in $4 \mathrm{~N} \mathrm{HCl}(2.00 \mathrm{~mL}, 0.4 \mathrm{M})$ and heated to $120{ }^{\circ} \mathrm{C}$ (oil bath temperature). After stirring for six hours at this temperature the mixture was allowed to cool to $25^{\circ} \mathrm{C}$ and saturated $\mathrm{NaHCO}_{3}$ solution $(10 \mathrm{~mL})$ and $\mathrm{CH}_{2} \mathrm{Cl}_{2}(10 \mathrm{~mL})$ were added. The phases were separated and the aqueous phase was extracted with $\mathrm{CH}_{2} \mathrm{Cl}_{2}(3 \times 10 \mathrm{~mL})$. The combined organic phases were washed with saturated $\mathrm{NaHCO}_{3}$ solution $(10 \mathrm{~mL})$, dried over anhydrous $\mathrm{Na}_{2} \mathrm{SO}_{4}$, filtered and concentrated under reduced pressure. Purification by flash column chromatography $\left(\mathrm{SiO}_{2}, \mathrm{CH}: \mathrm{EE} 6: 1 \mathrm{v}: \mathrm{v}\right)$ afforded $20(120 \mathrm{mg}, 365 \mu \mathrm{mol}, 91 \%)$ as a colorless solid.

$\mathbf{R}_{f}=0.22\left(\mathrm{SiO}_{2}\right.$, CH:EE 6:1 v:v). Mp.: $215-217^{\circ} \mathrm{C} .{ }^{1} \mathbf{H}-\mathbf{N M R}\left(\mathbf{3 6 0} \mathbf{M H z}, \mathbf{C D C l}_{\mathbf{3}}\right): \delta=7.94\left(\mathrm{~d},{ }^{5} \boldsymbol{J}_{\mathrm{HH}}=\right.$ $0.9 \mathrm{~Hz}, 1 \mathrm{H}), 7.75\left(\mathrm{~d},{ }^{5} J_{\mathrm{HH}}=0.8 \mathrm{~Hz}, 1 \mathrm{H}\right), 7.65\left(\mathrm{~d},{ }^{3} J_{\mathrm{HH}}=8.1 \mathrm{~Hz}, 1 \mathrm{H}\right), 7.62\left(\mathrm{~d},{ }^{4} J_{\mathrm{HH}}=1.8 \mathrm{~Hz}, 1 \mathrm{H}\right), 7.52$ $\left(\mathrm{dd},{ }^{3} J_{\mathrm{HH}}=8.1 \mathrm{~Hz},{ }^{4} J_{\mathrm{HH}}=1.8 \mathrm{~Hz}, 1 \mathrm{H}\right), 5.37(\mathrm{~s}, 2 \mathrm{H}), 1.51(\mathrm{~s}, 6 \mathrm{H}) \mathrm{ppm} .{ }^{13} \mathbf{C}\left\{{ }^{1} \mathbf{H}\right\}-\mathbf{N M R}\left(\mathbf{9 1} \mathbf{M H z}, \mathbf{C D C l}_{3}\right)$ : $\delta=171.4,156.9,154.9,146.7,144.9,136.3,130.8,126.8,125.0,123.5,122.5,120.1,113.4,69.8,47.1$, 27.1 (2x) ppm. IR (ATR, neat): $\tilde{v}=2910$ (w), 2869 (w), 2360 (w), 1755 (s), 1622 (m), 1452 (m), 1345 (s), 1252 (m), $1184(\mathrm{~s}), 1081(\mathrm{~m}), 1055$ (m), 1034 (s), 1006 (s), 953 (w), $884(\mathrm{~m}), 870$ (m), 822 (s), 777 
(s), $762(\mathrm{~s}) \mathrm{cm}^{-1}$. MS (APCI): $\mathrm{m} / \mathrm{z}=329.1\left[\mathrm{C}_{17} \mathrm{H}_{13}{ }^{79} \mathrm{BrO}_{2}+\mathrm{H}\right]^{+}$. HR-MS (EI, $\left.70 \mathrm{eV}\right)$ : calculated for $\mathrm{C}_{17} \mathrm{H}_{13}{ }^{79} \mathrm{BrO}_{2}{ }^{+\cdot}[\mathrm{M}]^{+*}: \mathrm{m} / \mathrm{z}=328.00934$, found: 328.00958 (Dev.: $0.24 \mathrm{mu} ; 0.72 \mathrm{ppm}$ ). The analytical data are in accordance with the literature.[23]

\subsubsection{2-(3-(Azidomethyl)-7-bromo-9,9-dimethyl-9H-fluoren-2-yl)-4,4-dimethyl-4,5- dihydrooxazole (21)}

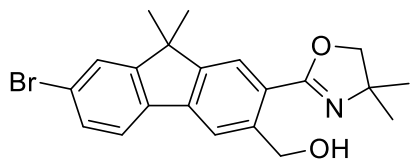

19

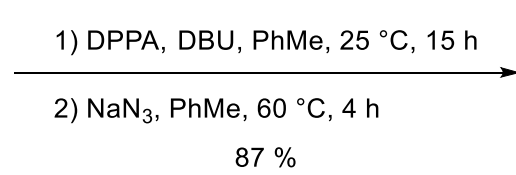

$87 \%$

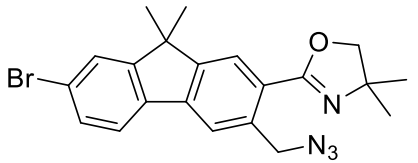

21

A modified literature procedure was used.[11] 19 (1.00 g, $2.50 \mathrm{mmol}, 1.00 \mathrm{eq})$ was dissolved in PhMe $(12.5 \mathrm{~mL}, 0.2 \mathrm{M})$ and DBU ( $485 \mu \mathrm{L}, 3.25 \mathrm{mmol}, 1.30 \mathrm{eq})$ was added via syringe pump $(0.5 \mathrm{~mL} / \mathrm{min})$ at $25^{\circ} \mathrm{C}$. The mixture was stirred for ten minutes, whereupon DPPA ( $\left.645 \mu \mathrm{L}, 3.00 \mathrm{mmol}, 1.20 \mathrm{eq}\right)$ was added via syringe pump $(0.3 \mathrm{~mL} / \mathrm{min})$. After complete addition the suspension was stirred for 15 hours at $25{ }^{\circ} \mathrm{C}$. Due to incomplete consumption of the intermediately formed phosphate, $\mathrm{NaN}_{3}(163 \mathrm{mg}$, $2.50 \mathrm{mmol}, 1.00 \mathrm{eq}$ ) was added and the mixture was heated to $60{ }^{\circ} \mathrm{C}$ (oil bath temperature) for four hours. Saturated $\mathrm{NH}_{4} \mathrm{Cl}$ solution $(10 \mathrm{~mL})$ and $\mathrm{CH}_{2} \mathrm{Cl}_{2}(30 \mathrm{~mL})$ were added, the phases separated and the aqueous phase was extracted with $\mathrm{CH}_{2} \mathrm{Cl}_{2}(4 \times 40 \mathrm{~mL})$. The combined organic phases were dried over anhydrous $\mathrm{Na}_{2} \mathrm{SO}_{4}$, filtered and the solvent was removed under reduced pressure. Purification by flash column chromatography ( $\left.\mathrm{SiO}_{2}, \mathrm{CH}: \mathrm{EE} 15: 1 \mathrm{v}: \mathrm{v}\right)$ provided $21(921 \mathrm{mg}, 2.17 \mathrm{mmol}, 87 \%)$ as a colorless oil. The oil solidified upon standing at $25{ }^{\circ} \mathrm{C}$ to form a colorless solid.

$\mathbf{R}_{f}=0.34\left(\mathrm{SiO}_{2}, \mathrm{CH}: \mathrm{EE} 15: 1 \mathrm{v}: \mathrm{v}\right) . \mathbf{M p} .: 122-124{ }^{\circ} \mathrm{C} .{ }^{1} \mathbf{H}-\mathbf{N M R}\left(\mathbf{3 6 0} \mathbf{M H z}, \mathbf{C D C l}_{\mathbf{3}}\right): \delta=7.94(\mathrm{~s}, 1 \mathrm{H})$, $7.75(\mathrm{~s}, 1 \mathrm{H}), 7.62\left(\mathrm{~d},{ }^{3} J_{\mathrm{HH}}=8.1 \mathrm{~Hz}, 1 \mathrm{H}\right), 7.57\left(\mathrm{~d},{ }^{4} J_{\mathrm{HH}}=1.8 \mathrm{~Hz}, 1 \mathrm{H}\right), 7.49\left(\mathrm{dd},{ }^{3} J_{\mathrm{HH}}=8.1 \mathrm{~Hz},{ }^{4} J_{\mathrm{HH}} 1.8\right.$ $\mathrm{Hz}, 1 \mathrm{H}), 4.95$ (s, 2H), 4.12 (s, 2H), 1.49 (s, 6H), 1.42 (s, 6H) ppm. ${ }^{13} \mathbf{C}\left\{{ }^{1} \mathbf{H}\right\}-\mathbf{N M R}$ (91 MHz, CDCl $)$ : $\delta$ $=161.5,156.6,152.8,141.0,137.1,135.8,130.5,126.4,126.0,124.6,122.4,122.2,121.3$, 78.7, 68.5, 53.6, 47.4, 28.5 (2x), 26.9 (2x) ppm. IR (ATR, neat): $\tilde{v}=2964$ (w), 2924 (w), 2359 (w), 2103 (s), 1637 (m), 1458 (m), 1409 (m), 1360 (m), 1327 (m), 1301 (s), 1261 (s), 1200 (m), 1087 (m), 1061 (m), 1038 (s), $974(\mathrm{~m}), 951$ (m), 908 (m), 872 (m), 812 (s), 767 (m), 742 (s) $\mathrm{cm}^{-1}$. MS (APCI): m/z = 425.2 $\left[\mathrm{C}_{21} \mathrm{H}_{21}{ }^{79} \mathrm{BrN}{ }_{4} \mathrm{O}+\mathrm{H}\right]^{+}$. HR-MS (APCI): calculated for $\mathrm{C}_{21} \mathrm{H}_{22}{ }^{79} \mathrm{BrN}_{4} \mathrm{O}^{+}[\mathrm{M}+\mathrm{H}]^{+}: \mathrm{m} / \mathrm{z}=425.09715$, found: 425.09753 (Dev.: $0.38 \mathrm{mu} ; 0.90 \mathrm{ppm}$ ). The analytical data are in accordance with the literature.[23] 


\subsubsection{3-(Azidomethyl)-7-bromo-9,9-dimethyl-9H-fluorene-2-carbaldehyde (5)}

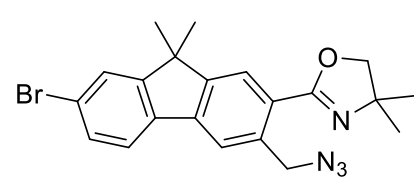

21

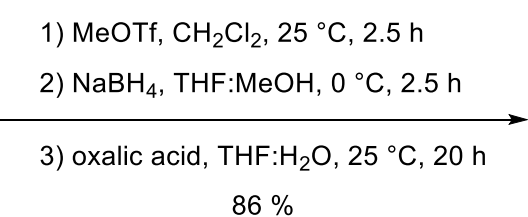

$86 \%$

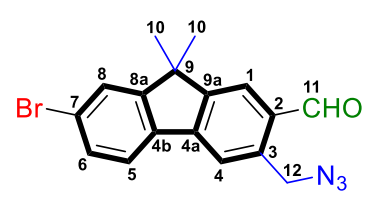

A modified literature procedure was used.[12] 21 (319 mg, $750 \mu$ mol, 1.00 eq) was dissolved in $\mathrm{CH}_{2} \mathrm{Cl}_{2}$ $(2.50 \mathrm{~mL}, 0.3 \mathrm{M})$ and MeOTf $(164 \mu \mathrm{L}, 1.50 \mathrm{mmol}, 2.00 \mathrm{eq})$ was added via syringe in one portion at $25{ }^{\circ} \mathrm{C}$. After stirring for two and a half hours at the same temperature the solution was cooled to $0{ }^{\circ} \mathrm{C}$. A solution of $\mathrm{NaBH}_{4}(56.8 \mathrm{mg}, 1.50 \mathrm{mmol}, 2.00 \mathrm{eq})$ in THF:MeOH (2.5 mL, 4:1 v:v, $\left.0.3 \mathrm{M}\right)$ was added via syringe within five minutes and stirring was continued at $0{ }^{\circ} \mathrm{C}$ for another two and a half hours. Saturated $\mathrm{NH}_{4} \mathrm{Cl}$ solution $(5 \mathrm{~mL})$, water $(5 \mathrm{~mL})$ and $\mathrm{CH}_{2} \mathrm{Cl}_{2}(10 \mathrm{~mL})$ were added and the phases were separated. The aqueous layer was extracted with $\mathrm{CH}_{2} \mathrm{Cl}_{2}(4 \times 20 \mathrm{~mL})$ and the combined organic phases were dried over anhydrous $\mathrm{Na}_{2} \mathrm{SO}_{4}$. After filtration and solvent evaporation under reduced pressure, the residue was redissolved in THF: $\mathrm{H}_{2} \mathrm{O}(2.5 \mathrm{~mL}, 4: 1 \mathrm{v}: \mathrm{v}, 0.3 \mathrm{M})$. Oxalic acid dihydrate $(189 \mathrm{mg}$, $1.50 \mathrm{mmol}, 2.00 \mathrm{eq}$ ) was added at $25^{\circ} \mathrm{C}$ and the mixture was stirred for 20 hours at the same temperature. Saturated $\mathrm{NaHCO}_{3}$ solution $(10 \mathrm{~mL})$, water $(10 \mathrm{~mL})$ and $\mathrm{CH}_{2} \mathrm{Cl}_{2}(20 \mathrm{~mL})$ were added and the phases separated. The aqueous layer was extracted with $\mathrm{CH}_{2} \mathrm{Cl}_{2}(4 \times 20 \mathrm{~mL})$, the combined organic phases were dried over anhydrous $\mathrm{Na}_{2} \mathrm{SO}_{4}$, filtered and the solvent was removed under reduced pressure. Purification by flash column chromatography ( $\left.\mathrm{SiO}_{2}, \mathrm{CH}: \mathrm{EE} 20: 1 \mathrm{v}: \mathrm{v}\right)$ afforded 5 ( $230 \mathrm{mg}, 646 \mu \mathrm{mol}$, $86 \%$ ) as a colorless oil. Upon standing at $25{ }^{\circ} \mathrm{C}$ the oil solidified very slowly to form a colorless solid. Single crystals suitable for $\mathrm{X}$-ray analysis were grown by slow diffusion of cyclohexane into a $\mathrm{CH}_{2} \mathrm{Cl}_{2}$ solution.

$\mathbf{R}_{f}=0.27\left(\mathrm{SiO}_{2}, \mathrm{CH}: \mathrm{EE} 20: 1 \mathrm{v}: \mathrm{v}\right)$. Mp.: $103-105{ }^{\circ} \mathrm{C} .{ }^{\mathbf{1}} \mathbf{H}-\mathbf{N M R}\left(\mathbf{3 6 0} \mathbf{M H z}, \mathbf{C D C l}_{3}\right): \delta=10.20(\mathrm{~s}, 1 \mathrm{H}$, $\mathrm{CHO}), 7.90(\mathrm{~s}, 1 \mathrm{H}, \mathrm{H}-1), 7.84(\mathrm{~s}, 1 \mathrm{H}, \mathrm{H}-4), 7.69\left(\mathrm{~d},{ }^{3} J_{\mathrm{HH}}=8.1 \mathrm{~Hz}, 1 \mathrm{H}, \mathrm{H}-5\right), 7.62\left(\mathrm{~d},{ }^{4} J_{\mathrm{HH}}=1.7 \mathrm{~Hz}\right.$, $1 \mathrm{H}, \mathrm{H}-8), 7.53\left(\mathrm{dd},{ }^{3} J_{\mathrm{HH}}=8.1 \mathrm{~Hz},{ }^{4} J_{\mathrm{HH}}=1.8 \mathrm{~Hz}, 1 \mathrm{H}, \mathrm{H}-6\right), 4.95\left(\mathrm{~s}, 2 \mathrm{H}, \mathrm{CH}_{2}\right), 1.53\left(\mathrm{~s}, 6 \mathrm{H}, 2 \mathrm{xCH}_{3}\right) \mathrm{ppm}$. ${ }^{13} \mathbf{C}\left\{{ }^{1} \mathbf{H}\right\}$-NMR (91 MHz, $\left.\mathbf{C D C l}_{3}\right): \delta=192.3$ (C-11), 157.1 (C-8a), 153.3 (C-9a), 144.0 (C-4a), 137.5 (C-3), 136.5 (C-4b), 132.7 (C-2), 130.9 (C-6), 128.4 (C-1), 126.7 (C-8), 123.5 (C-7), 122.9 (C-5), 121.2 (C-4), 52.5 (C-12), 47.4 (C-9), 26.8 (2x, C-10) ppm. IR (ATR, neat): $\tilde{v}=2965(\mathrm{w}), 2929(\mathrm{w})$, 2860 (w), 2735 (w), 2359 (w), 2103 (s), 1682 (s), 1616 (m), 1599 (m) 1558 (s), 1452 (m), 1425 (m), 1361 (m), 1294 (m), 1254 (m), 1178 (s), 1151 (s), 1060 (m), 965 (m), 887 (m), 877 (m), 828 (s), 796 (s), $774(\mathrm{~m}), 746$ (s), 737 (s), $674(\mathrm{~m}) \mathrm{cm}^{-1}$. MS (EI, 70 eV): m/z = $354.9\left[\mathrm{C}_{17} \mathrm{H}_{14}{ }^{79} \mathrm{Br}\right]^{++}$. HR-MS (EI, $70 \mathrm{eV}$ ): calculated for $\mathrm{C}_{17} \mathrm{H}_{14}{ }^{79} \mathrm{BrN}_{3} \mathrm{O}^{+\cdot}[\mathrm{M}]^{+*}: \mathrm{m} / \mathrm{z}=355.03148$, found: 355.03133 (Dev.: $-0.15 \mathrm{mu}$; $-0.41 \mathrm{ppm})$. The analytical data are in accordance with the literature.[23] 


\subsubsection{2-(7-Bromo-3-(methoxymethyl)-9,9-dimethyl-9H-fluoren-2-yl)-4,4-dimethyl-4,5- dihydrooxazole (22)}

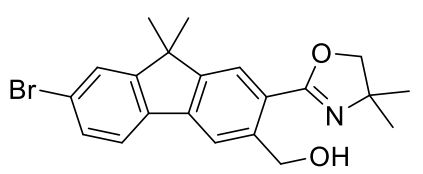

19

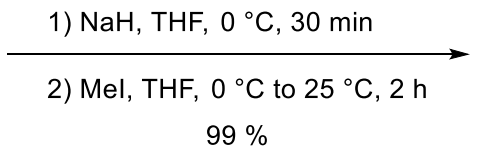

$99 \%$

A slightly modified literature procedure was used.[25] A heat gun-dried and nitrogen-flushed Schlenk tube, equipped with a magnetic stirring bar and a rubber septum, was charged with 19 (200 mg, $500 \mu \mathrm{mol}, 1.00 \mathrm{eq})$. The tube was flushed with nitrogen, anhydrous THF $(5.00 \mathrm{~mL}, 0.1 \mathrm{M})$ was added and the colorless solution was cooled to $0{ }^{\circ} \mathrm{C}$. $\mathrm{NaH}(40.0 \mathrm{mg}, 1.00 \mathrm{mmol}, 2.00 \mathrm{eq}, 60 \%$ in mineral oil) was added at once and the mixture was stirred at $0{ }^{\circ} \mathrm{C}$ for 30 minutes. $\mathrm{MeI}(68.5 \mu \mathrm{L}, 156 \mathrm{mg}, 1.10 \mathrm{mmol}$, $2.20 \mathrm{eq}$ ) was dropwise added via syringe at $0{ }^{\circ} \mathrm{C}$ and the mixture was stirred for two hours while it was allowed to warm to $25^{\circ} \mathrm{C}$. Saturated $\mathrm{NH}_{4} \mathrm{Cl}$ solution $(10 \mathrm{~mL})$ and $\mathrm{CH}_{2} \mathrm{Cl}_{2}(10 \mathrm{~mL})$ were added and the phases were separated. The aqueous phase was extracted with $\mathrm{CH}_{2} \mathrm{Cl}_{2}(4 \times 10 \mathrm{~mL})$ and the combined organic phases were dried over anhydrous $\mathrm{Na}_{2} \mathrm{SO}_{4}$, filtered and the solvent was removed under reduced

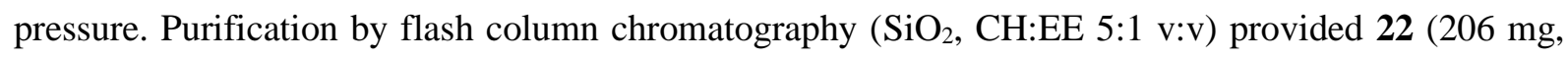
$497 \mathrm{mmol}, 99 \%$ ) as a colorless oil, which solidified rapidly upon standing at $25{ }^{\circ} \mathrm{C}$.

$\mathbf{R}_{f}=0.34\left(\mathrm{SiO}_{2}, \mathrm{CH}: \mathrm{EE} 5: 1 \mathrm{v}: \mathrm{v}\right) . \mathbf{M p} .: 151-153{ }^{\circ} \mathrm{C} .{ }^{1} \mathbf{H}-\mathbf{N M R}\left(\mathbf{6 0 1} \mathbf{M H z}, \mathbf{C D C l}_{3}\right): \delta=7.90(\mathrm{~s}, 1 \mathrm{H})$, $7.84(\mathrm{~s}, 1 \mathrm{H}), 7.63\left(\mathrm{~d},{ }^{3} J_{\mathrm{HH}}=8.1 \mathrm{~Hz}, 1 \mathrm{H}\right), 7.56\left(\mathrm{~d},{ }^{4} J_{\mathrm{HH}}=1.7 \mathrm{~Hz}, 1 \mathrm{H}\right), 7.47\left(\mathrm{dd},{ }^{3} J_{\mathrm{HH}}=8.1 \mathrm{~Hz},{ }^{4} J_{\mathrm{HH}}=1.8\right.$ $\mathrm{Hz}, 1 \mathrm{H}), 4.88$ (s, 2H), 4.10 (s, 2H), 3.50 (s, 3H), 1.48 (s, 6H), 1.41 (s, 6H) ppm. ${ }^{13} \mathbf{C}\left\{{ }^{1} \mathbf{H}\right\}-\mathbf{N M R}$ (151 MHz, $\left.\mathbf{C D C l}_{3}\right): \delta=162.1,156.6,151.8,140.7,138.8,137.6,130.4,126.4,125.5,124.1,122.2$, 122.0, 119.4, 78.7, 73.0, 68.3, 58.8, 47.3, 28.6 (2x), 27.0 (2x) ppm. IR (ATR, neat): $\tilde{v}=2960$ (w), 2921 (w), 2896 (w), 2804 (w), 1640 (m), 1601 (w), 1566 (w), 1483 (w), 1458 (w), 1430 (w), 1411 (w), 1384 (w), 1355 (m), 1302 (w), 1285 (w), 1262 (m), 1191 (m), 1161 (m), 1110 (s), $1084(\mathrm{~m}), 1060$ (m), 1035 (s), $971(\mathrm{~m}), 945$ (m), $921(\mathrm{~m}), 869(\mathrm{~m}), 901(\mathrm{~m}), 882(\mathrm{~m}), 820(\mathrm{~m}), 809$ (s), $765(\mathrm{~m}), 742(\mathrm{~m}), 672(\mathrm{w})$ $\mathrm{cm}^{-1}$. MS (ESI): $\mathrm{m} / \mathrm{z}=413.9\left[\mathrm{C}_{22} \mathrm{H}_{24}{ }^{79} \mathrm{BrNO}_{2}+\mathrm{H}\right]^{+}$. HR-MS (ESI): calculated for $\mathrm{C}_{22} \mathrm{H}_{25}{ }^{79} \mathrm{BrNO}_{2}{ }^{+}$ $[\mathrm{M}+\mathrm{H}]^{+}: \mathrm{m} / \mathrm{z}=414.10632$, found: 414.10617 (Dev.: $-0.15 \mathrm{mu} ;-0.35 \mathrm{ppm}$ ); calculated for $\mathrm{C}_{22} \mathrm{H}_{24}{ }^{79} \mathrm{BrNNaO}_{2}{ }^{+}[\mathrm{M}+\mathrm{Na}]^{+}: \mathrm{m} / \mathrm{z}=436.08826$, found: 436.08811 (Dev.: $-0.15 \mathrm{mu} ;-0.34 \mathrm{ppm}$ ).

\subsubsection{7-Bromo-3-(methoxymethyl)-9,9-dimethyl-9H-fluorene-2-carbaldehyde (23)}

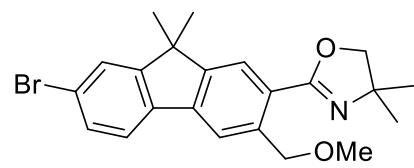

22

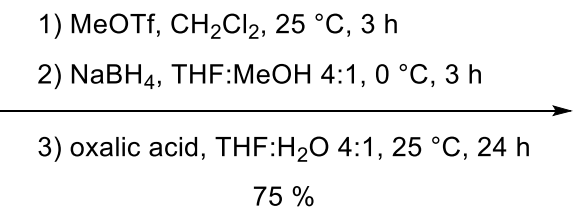

$75 \%$

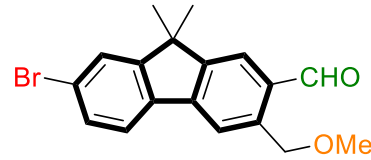

23

A modified literature procedure was used.[12] $22(82.9 \mathrm{mg}, 200 \mu \mathrm{mol}, 1.00 \mathrm{eq})$ was dissolved in $\mathrm{CH}_{2} \mathrm{Cl}_{2}$ $(1.00 \mathrm{~mL}, 0.2 \mathrm{M})$ and MeOTf $(43.8 \mu \mathrm{L}, 400 \mu \mathrm{mol}, 2.00 \mathrm{eq})$ was added via syringe in one portion at 
$25{ }^{\circ} \mathrm{C}$. After stirring for three hours at the same temperature the solution was cooled to $0{ }^{\circ} \mathrm{C}$. A solution of $\mathrm{NaBH}_{4}(15.1 \mathrm{mg}, 400 \mu \mathrm{mol}, 2.00 \mathrm{eq})$ in THF:MeOH $(1.0 \mathrm{~mL}, 4: 1 \mathrm{v}: \mathrm{v}, 0.4 \mathrm{M})$ was added via syringe within five minutes and stirring was continued at $0{ }^{\circ} \mathrm{C}$ for another three hours. Saturated $\mathrm{NH}_{4} \mathrm{Cl}$ solution $(5 \mathrm{~mL})$, water $(5 \mathrm{~mL})$ and $\mathrm{CH}_{2} \mathrm{Cl}_{2}(10 \mathrm{~mL})$ were added and the phases were separated. The aqueous layer was extracted with $\mathrm{CH}_{2} \mathrm{Cl}_{2}(4 \times 10 \mathrm{~mL})$ and the combined organic phases were dried over anhydrous $\mathrm{Na}_{2} \mathrm{SO}_{4}$. After filtration and solvent evaporation under reduced pressure, the residue was redissolved in THF: $\mathrm{H}_{2} \mathrm{O}$ (1.0 mL, 4:1 v:v, 0.2 M). Oxalic acid dihydrate (50.4 mg, $\left.400 \mu \mathrm{mol}, 2.00 \mathrm{eq}\right)$ was added at $25{ }^{\circ} \mathrm{C}$ and the mixture was stirred for 24 hours under the same conditions. Saturated $\mathrm{NaHCO}_{3}$ solution $(5 \mathrm{~mL})$, water $(5 \mathrm{~mL})$ and $\mathrm{CH}_{2} \mathrm{Cl}_{2}(10 \mathrm{~mL})$ were added and the phases were separated. The aqueous layer was extracted with $\mathrm{CH}_{2} \mathrm{Cl}_{2}(4 \times 10 \mathrm{~mL})$, the combined organic phases were dried over anhydrous $\mathrm{Na}_{2} \mathrm{SO}_{4}$, filtered and the solvent was removed under reduced pressure. Purification by flash column chromatography ( $\left.\mathrm{SiO}_{2}, \mathrm{CH}: \mathrm{EE} 10: 1 \mathrm{v}: \mathrm{v}\right)$ afforded $23(51.6 \mathrm{mg}, 150 \mu \mathrm{mol}, 75 \%)$ as a colorless solid.

$\mathbf{R}_{f}=0.18\left(\mathrm{SiO}_{2}, \mathrm{CH}: \mathrm{EE}\right.$ 10:1 v:v). Mp.: $62-64{ }^{\circ} \mathrm{C} .{ }^{1} \mathbf{H}-\mathrm{NMR}\left(\mathbf{6 0 0} \mathbf{M H z}, \mathbf{C D C l}_{3}\right): \delta=10.26(\mathrm{~s}, 1 \mathrm{H})$, $7.92(\mathrm{~s}, 1 \mathrm{H}), 7.91(\mathrm{~s}, 1 \mathrm{H}), 7.68\left(\mathrm{~d},{ }^{3} J_{\mathrm{HH}}=8.1 \mathrm{~Hz}, 1 \mathrm{H}\right), 7.61\left(\mathrm{~d},{ }^{4} J_{\mathrm{HH}}=1.6 \mathrm{~Hz}, 1 \mathrm{H}\right), 7.51\left(\mathrm{dd},{ }^{3} J_{\mathrm{HH}}=8.1\right.$ $\left.\mathrm{Hz},{ }^{4} \mathrm{~J}_{\mathrm{HH}}=1.7 \mathrm{~Hz}, 1 \mathrm{H}\right), 4.93$ (s, 2H), $3.54(\mathrm{~s}, 3 \mathrm{H}), 1.51$ (s, 6H) ppm. ${ }^{13} \mathbf{C}\left\{{ }^{1} \mathbf{H}\right\}-\mathbf{N M R}\left(\mathbf{1 5 1} \mathbf{~ M H z}, \mathbf{C D C l}_{3}\right)$ : $\delta=192.2,157.2,152.6,143.8,141.0,136.9,132.8,130.7,126.6,126.2,123.2,122.8,120.0,72.2,58.9$, 47.4, 26.9 (2x) ppm. IR (ATR, neat): $\tilde{v}=2956$ (w), 2923 (w), 2864 (w), 2820 (w), 2721 (w), 1682 (s), 1614 (m), 1598 (m), 1559 (s), 1471 (m), 1444 (m), 1405 (m), 1384 (m), 1361 (w), 1334 (m), 1302 (w), $1282(\mathrm{w}), 1245(\mathrm{~m}), 1222$ (w), $1178(\mathrm{~s}), 1156(\mathrm{~m}), 1134(\mathrm{~m}), 1104(\mathrm{~s}), 1079(\mathrm{~m}), 1057(\mathrm{~m}), 980$ (s), 907 (m), 886 (m), 838 (m), 825 (s), 798 (s), 772 (m), 729 (s), 673 (m) cm $\mathrm{cm}^{-1}$ MS (ESI): m/z = 413.9 $\left[\mathrm{C}_{22} \mathrm{H}_{24}{ }^{79} \mathrm{BrNO}_{2}+\mathrm{H}\right]^{+}$. HR-MS (ESI): calculated for $\mathrm{C}_{18} \mathrm{H}_{17}{ }^{79} \mathrm{BrNaO}_{2}{ }^{+}[\mathrm{M}+\mathrm{Na}]^{+}: \mathrm{m} / \mathrm{z}=367.03041$, found: 367.03027 (Dev.: $-0.14 \mathrm{mu} ;-0.39 \mathrm{ppm}$ ); calculated for $\mathrm{C}_{36} \mathrm{H}_{34}{ }^{79} \mathrm{Br}_{2} \mathrm{NaO}_{4}{ }^{+}[2 \mathrm{M}+\mathrm{Na}]^{+}: \mathrm{m} / \mathrm{z}=$ 711.07160, found: 711.07146 (Dev.: $-0.14 \mathrm{mu} ;-0.20 \mathrm{ppm}$ ).

\subsubsection{9-Bromo-3,3,11,11-tetramethyl-2,3,5,11-tetrahydroindeno[1,2-f]oxazolo[2,3- a]isoindol-4-ium bromide (25)}

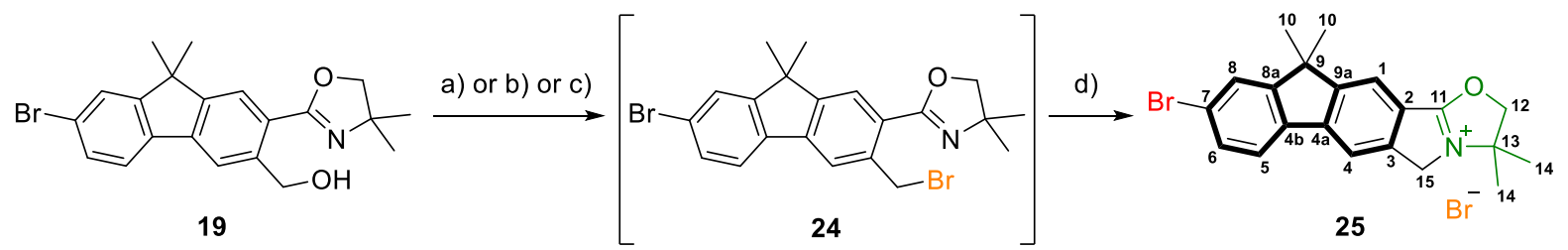

Reaction conditions: a) $\mathrm{CBr}_{4}, \mathrm{PPh}_{3}, \mathrm{CH}_{2} \mathrm{Cl}_{2}, 0{ }^{\circ} \mathrm{C}$ to $25^{\circ} \mathrm{C}, 2 \mathrm{~h}, 95 \%$; b) $\mathrm{NBS}, \mathrm{PPh}_{3}, \mathrm{CH}_{2} \mathrm{Cl}_{2}, 0{ }^{\circ} \mathrm{C}$ to $25{ }^{\circ} \mathrm{C}, 2 \mathrm{~h}$, $91 \%$; c) $\mathrm{PBr}_{3}, \mathrm{CH}_{2} \mathrm{Cl}_{2}, 0{ }^{\circ} \mathrm{C}$ to $25^{\circ} \mathrm{C}, 2 \mathrm{~h}, 92 \%$; d) spontaneous during/upon workup.

For a): A slightly modified literature procedure was used.[26] A heat gun-dried and nitrogen-flushed Schlenk tube, equipped with a magnetic stirring bar and a rubber septum, was charged with $\mathbf{1 9}(80.1 \mathrm{mg}$, 
$200 \mu \mathrm{mol}, 1.00 \mathrm{eq})$. The tube was flushed with nitrogen, anhydrous $\mathrm{CH}_{2} \mathrm{Cl}_{2}(2.00 \mathrm{~mL}, 0.1 \mathrm{M})$ was added and the colorless solution was cooled to $0{ }^{\circ} \mathrm{C}$. $\mathrm{PPh}_{3}(78.7 \mathrm{mg}, 300 \mu \mathrm{mol}, 1.50 \mathrm{eq})$ and $\mathrm{CBr}_{4}(99.5 \mathrm{mg}$, $300 \mu \mathrm{mol}, 1.50 \mathrm{eq})$ were added subsequently and the mixture was stirred for two hours while it was allowed to warm to $25^{\circ} \mathrm{C}$ - the initial dark yellow color disappeared in the course of the reaction. After full conversion the reaction mixture was directly flushed through a plug of neutral alumina using $\mathrm{CH}_{2} \mathrm{Cl}_{2}$. The solvent was removed under reduced pressure to afford the cyclized iminium bromide 25 (88.3 mg, $191 \mu \mathrm{mol}, 95 \%$ ) as a colorless foam.

For b): A slightly modified literature procedure was used.[27] A heat gun-dried and nitrogen-flushed Schlenk tube, equipped with a magnetic stirring bar and a rubber septum, was charged with 19 (80.1 mg, $200 \mu \mathrm{mol}, 1.00 \mathrm{eq})$. The tube was flushed with nitrogen, anhydrous $\mathrm{CH}_{2} \mathrm{Cl}_{2}(2.00 \mathrm{~mL}, 0.1 \mathrm{M})$ was added and the colorless solution was cooled to $0{ }^{\circ} \mathrm{C}$. $\mathrm{PPh}_{3}(78.7 \mathrm{mg}, 300 \mu \mathrm{mol}, 1.50 \mathrm{eq})$ and NBS (53.4 mg, $300 \mu \mathrm{mol}, 1.50 \mathrm{eq})$ were added subsequently and the mixture was stirred for two hours while it was allowed to warm to $25^{\circ} \mathrm{C}$ - the mixture stayed slightly yellow in the course of the reaction. After full conversion the reaction mixture was directly flushed through a plug of neutral alumina using $\mathrm{CH}_{2} \mathrm{Cl}_{2}$. The solvent was removed under reduced pressure to afford the cyclized iminium bromide $\mathbf{2 5}(84.5 \mathrm{mg}$, $182 \mu \mathrm{mol}, 91 \%)$ as a colorless foam.

For c): A slightly modified literature procedure was used.[28] A heat gun-dried and nitrogen-flushed Schlenk tube, equipped with a magnetic stirring bar and a rubber septum, was charged with $\mathbf{1 9}(80.1 \mathrm{mg}$, $200 \mu \mathrm{mol}, 1.00 \mathrm{eq})$. The tube was flushed with nitrogen, anhydrous $\mathrm{CH}_{2} \mathrm{Cl}_{2}(2.00 \mathrm{~mL}, 0.1 \mathrm{M})$ was added and the colorless solution was cooled to $0{ }^{\circ} \mathrm{C}$. $\mathrm{PBr}_{3}(28.5 \mathrm{~mL}, 81.2 \mathrm{mg}, 300 \mu \mathrm{mol}, 1.50 \mathrm{eq})$ was added dropwise and the mixture was stirred for two hours while it was allowed to warm to $25^{\circ} \mathrm{C}$ - the mixture stayed colorless in the course of the reaction. After full conversion the reaction mixture was directly flushed through a plug of neutral alumina using $\mathrm{CH}_{2} \mathrm{Cl}_{2}$. The solvent was removed under reduced pressure to afford the cyclized iminium bromide 25 (85.2 $\mathrm{mg}, 184 \mu \mathrm{mol}, 92 \%)$ as a colorless foam.

24: $\mathbf{R}_{f}=0.44\left(\mathrm{SiO}_{2}, \mathrm{CH}: \mathrm{EE}\right.$ 6:1 v:v). HR-MS (ESI): calculated for $\mathrm{C}_{21} \mathrm{H}_{22}{ }^{79} \mathrm{Br}_{2} \mathrm{NO}^{+}[\mathrm{M}+\mathrm{H}]^{+}: \mathrm{m} / \mathrm{z}=$ 462.00626, found: 462.00581 (Dev.: $-0.46 \mathrm{mu} ;-0.99 \mathrm{ppm}$ ); calculated for $\mathrm{C}_{21} \mathrm{H}_{21}{ }^{79} \mathrm{Br}_{2} \mathrm{NNaO}^{+}[\mathrm{M}+\mathrm{Na}]^{+}$: $\mathrm{m} / \mathrm{z}=483.98821$, found: 483.98779 (Dev.: $-0.42 \mathrm{mu} ;-0.87 \mathrm{ppm}$ ); calculated for $\mathrm{C}_{21} \mathrm{H}_{21}{ }^{79} \mathrm{Br}_{2} \mathrm{KNO}^{+}$ $[\mathrm{M}+\mathrm{K}]^{+}: \mathrm{m} / \mathrm{z}=499.96215$, found: 499.96202 (Dev.: $-0.13 \mathrm{mu} ;-0.26 \mathrm{ppm}$ ); calculated for $\mathrm{C}_{42} \mathrm{H}_{43}{ }^{79} \mathrm{Br}_{4} \mathrm{~N}_{2} \mathrm{O}_{2}{ }^{+}[2 \mathrm{M}+\mathrm{H}]^{+}: \mathrm{m} / \mathrm{z}=923.00525$, found: 923.00458 (Dev.: $-0.67 \mathrm{mu} ;-0.73 \mathrm{ppm}$ ); calculated for $\mathrm{C}_{42} \mathrm{H}_{42}{ }^{79} \mathrm{Br}_{4} \mathrm{~N}_{2} \mathrm{NaO}_{2}{ }^{+}[2 \mathrm{M}+\mathrm{Na}]^{+}: \mathrm{m} / \mathrm{z}=944.98720$, found: 944.98614 (Dev.: $-1.06 \mathrm{mu}$;$1.12 \mathrm{ppm}$ ); calculated for $\mathrm{C}_{42} \mathrm{H}_{42}{ }^{79} \mathrm{Br}_{4} \mathrm{KN}_{2} \mathrm{O}_{2}{ }^{+}[2 \mathrm{M}+\mathrm{K}]^{+}: \mathrm{m} / \mathrm{z}=960.96114$, found: 960.96237 (Dev.: 1.24 $\mathrm{mu} ; 1.29 \mathrm{ppm})$.

25: $\mathbf{R}_{f}=0.18\left(\mathrm{SiO}_{2}\right.$, CH:EE 6:1 v:v). Mp.: $108-110{ }^{\circ} \mathrm{C} .{ }^{1} \mathbf{H}-\mathrm{NMR}\left(601 \mathrm{MHz}, \mathbf{C D C l}_{3}\right): \delta=7.85$ (s, $1 \mathrm{H}, \mathrm{H}-1), 7.69(\mathrm{~s}, 1 \mathrm{H}, \mathrm{H}-4), 7.61\left(\mathrm{~d},{ }^{3} J_{\mathrm{HH}}=8.1 \mathrm{~Hz}, 1 \mathrm{H}, \mathrm{H}-5\right), 7.59\left(\mathrm{~d},{ }^{4} J_{\mathrm{HH}}=1.7 \mathrm{~Hz}, 1 \mathrm{H}, \mathrm{H}-8\right), 7.49$ 
$\left(\mathrm{dd},{ }^{3} J_{\mathrm{HH}}=8.1 \mathrm{~Hz},{ }^{4} J_{\mathrm{HH}}=1.8 \mathrm{~Hz}, 1 \mathrm{H}, \mathrm{H}-6\right), 4.60(\mathrm{~s}, 2 \mathrm{H}, \mathrm{H}-15), 4.16(\mathrm{~s}, 2 \mathrm{H}, \mathrm{H}-12), 1.69(\mathrm{~s}, 6 \mathrm{H}, \mathrm{H}-14)$, $1.50(\mathrm{~s}, 6 \mathrm{H}, \mathrm{H}-10)$ ppm. ${ }^{13} \mathrm{C}\left\{{ }^{1} \mathrm{H}\right\}-N M R\left(151 \mathbf{M H z}, \mathbf{C D C l}_{3}\right): \delta=169.2\left(\mathrm{C}=\mathrm{N}^{+}\right), 156.6(\mathrm{C}-8 \mathrm{a}), 153.8$ (C-9a), 142.0 (C-4a), 140.6 (C-3), 137.2 (C-4b), 133.2 (C-2), 130.5 (C-6), 126.6 (C-8), 122.4 (C-7), 122.0 (C-5), 117.8 (C-1), 114.0 (C-4), 56.7 (C-13), 50.0 (C-15), 47.1 (C-9), 41.7 (C-12), 27.2 (C10), 25.4 (C-14) ppm. IR (ATR, neat): $\tilde{v}=2960$ (w), 2922 (w), 2860 (w), 1669 (s), 1622 (m), 1486 (w), $1436(\mathrm{~m}), 1388$ (s), 1362 (m), 1314 (w), 1247 (s), 1200 (s), 1182 (m), 1142 (m), 1088 (m), 1065 (m), $976(\mathrm{w}), 951$ (w), 875 (m), 822 (s), 780 (s), 765 (m), 737 (m), $703(\mathrm{~m}), 660(\mathrm{~m}) \mathrm{cm}^{-1}$. MS (ESI): $\mathrm{m} / \mathrm{z}=382.0\left[\mathrm{C}_{21} \mathrm{H}_{21}{ }^{79} \mathrm{BrNO}\right]^{+}$. HR-MS (ESI): calculated for $\mathrm{C}_{21} \mathrm{H}_{21}{ }^{79} \mathrm{BrNO}^{+}\left[\mathrm{M}-\mathrm{Br}^{-}\right]^{+}: \mathrm{m} / \mathrm{z}=$ 382.08010, found: 382.07985 (Dev.: $-0.25 \mathrm{mu} ;-0.65 \mathrm{ppm}$ ).

\subsubsection{9-Bromo-3,3,4,11,11-pentamethyl-2,3,4,5,11,12b-hexahydroindeno[1,2- f] oxazolo[2,3-a]isoindol-4-ium trifluoromethanesulfonate (28)}
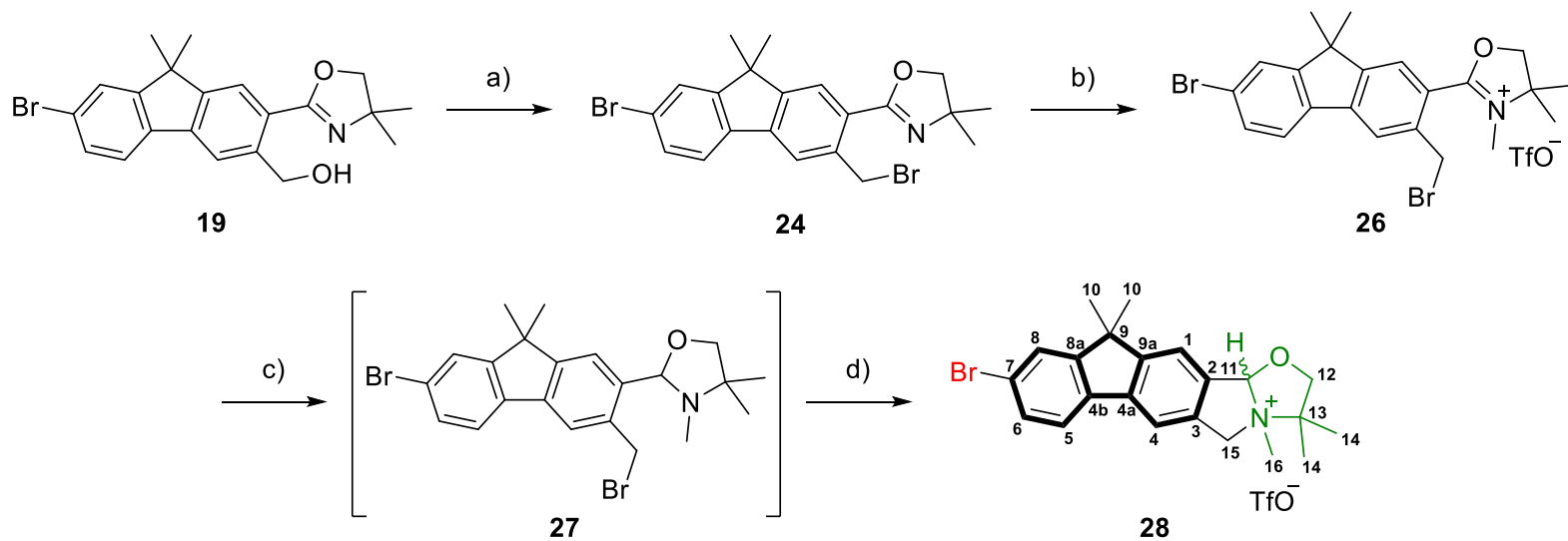

Reaction conditions: a) $\mathrm{CBr}_{4}, \mathrm{PPh}_{3}, \mathrm{CH}_{2} \mathrm{Cl}_{2}, 0{ }^{\circ} \mathrm{C}$ to $25{ }^{\circ} \mathrm{C}, 2 \mathrm{~h}$, rapid filtration over neutral alumina b) MeOTf, $\mathrm{CH}_{2} \mathrm{Cl}_{2}, 25{ }^{\circ} \mathrm{C}, 3 \mathrm{~h}$; c) $\mathrm{NaBH}_{4}$, THF:MeOH 4:1 v:v, $0{ }^{\circ} \mathrm{C}, 3 \mathrm{~h}$; d) during reaction, $56 \%$ from 19.

Slightly modified literature procedures were used.[12, 26] A heat gun-dried and nitrogen-flushed Schlenk tube, equipped with a magnetic stirring bar and a rubber septum, was charged with $19(160 \mathrm{mg}$, $400 \mu \mathrm{mol}, 1.00 \mathrm{eq})$. The tube was flushed with nitrogen, anhydrous $\mathrm{CH}_{2} \mathrm{Cl}_{2}(2.00 \mathrm{~mL}, 0.1 \mathrm{M})$ was added and the colorless solution was cooled to $0{ }^{\circ} \mathrm{C}$. $\mathrm{PPh}_{3}(157 \mathrm{mg}, 600 \mu \mathrm{mol}, 1.50 \mathrm{eq})$ and $\mathrm{CBr}_{4}(199 \mathrm{mg}, 600$ $\mu \mathrm{mol}, 1.50 \mathrm{eq}$ ) were added subsequently and the mixture was stirred for two hours while it was allowed to warm to $25^{\circ} \mathrm{C}$ - the initial dark yellow color disappeared in the course of the reaction. After full conversion the reaction mixture was rapidly flushed through a plug of neutral alumina using $\mathrm{CH}_{2} \mathrm{Cl}_{2}$. The solvent was removed under reduced pressure on a rotary evaporator $\left(30{ }^{\circ} \mathrm{C}\right.$ water bath temperature) to obtain primarily the benzyl bromide $\mathbf{2 4}$ as a colorless foam.

The crude was dissolved in $\mathrm{CH}_{2} \mathrm{Cl}_{2}(2.00 \mathrm{~mL}, 0.2 \mathrm{M})$ and $\mathrm{MeOTf}(87.5 \mu \mathrm{L}, 800 \mu \mathrm{mol}, 2.00 \mathrm{eq})$ was added via syringe in one portion at $25{ }^{\circ} \mathrm{C}$. After stirring for three hours at the same temperature the solution was cooled to $0{ }^{\circ} \mathrm{C}$. A solution of $\mathrm{NaBH}_{4}(30.3 \mathrm{mg}, 800 \mu \mathrm{mol}, 2.00 \mathrm{eq})$ in THF:MeOH $(1.0 \mathrm{~mL}$, $4: 1 \mathrm{v}: \mathrm{v}, 0.4 \mathrm{M}$ ) was added via syringe within five minutes and stirring was continued at $0{ }^{\circ} \mathrm{C}$ for another 
three hours. Saturated $\mathrm{NH}_{4} \mathrm{Cl}$ solution $(5 \mathrm{~mL})$, water $(5 \mathrm{~mL})$ and $\mathrm{CH}_{2} \mathrm{Cl}_{2}(10 \mathrm{~mL})$ were added and the phases were separated. The aqueous layer was extracted with $\mathrm{CH}_{2} \mathrm{Cl}_{2}(4 \times 10 \mathrm{~mL})$ and the combined organic phases were dried over anhydrous $\mathrm{Na}_{2} \mathrm{SO}_{4}$, filtered and the solvent was removed under reduced pressure. Due to unexpected cyclization a further acidic hydrolysis using oxalic acid becomes impossible. Purification by flash column chromatography $\left(\mathrm{SiO}_{2}, \mathrm{CH}_{2} \mathrm{Cl}_{2}: \mathrm{MeOH} 15: 1 \mathrm{v}: \mathrm{v}\right)$ afforded the ammonium triflate $28(87.7 \mathrm{mg}, 223 \mu \mathrm{mol}, 56 \%$ from 19) as a colorless foam.

$\mathbf{R}_{\boldsymbol{f}}=0.14\left(\mathrm{SiO}_{2}, \mathrm{CH}_{2} \mathrm{Cl}_{2}: \mathrm{MeOH}\right.$ 15:1 v:v). Mp.: $205-222^{\circ} \mathrm{C}$ (very long decomp.). ${ }^{1} \mathbf{H}-\mathbf{N M R}(\mathbf{6 0 1} \mathbf{M H z}$, MeOD- $\left.d_{4}\right): \delta=7.86(\mathrm{~s}, 1 \mathrm{H}, \mathrm{H}-4), 7.77\left(\mathrm{~d},{ }^{3} J_{\mathrm{HH}}=8.1 \mathrm{~Hz}, 1 \mathrm{H}, \mathrm{H}-5\right), 7.73(\mathrm{~s}, 1 \mathrm{H}, \mathrm{H}-1), 7.72\left(\mathrm{~d},{ }^{4} J_{\mathrm{HH}}=\right.$ $1.7 \mathrm{~Hz}, 1 \mathrm{H}, \mathrm{H}-8), 7.54\left(\mathrm{dd},{ }^{3} J_{\mathrm{HH}}=8.1 \mathrm{~Hz},{ }^{4} J_{\mathrm{HH}}=1.6 \mathrm{~Hz}, 1 \mathrm{H}, \mathrm{H}-6\right), 6.56(\mathrm{~s}, 1 \mathrm{H}, \mathrm{H}-11), 5.43\left(\mathrm{~d},{ }^{2} J_{\mathrm{HH}}=\right.$ $14.9 \mathrm{~Hz}, 1 \mathrm{H}, \mathrm{H}-15), 4.73\left(\mathrm{~d},{ }^{2} J_{\mathrm{HH}}=14.9 \mathrm{~Hz}, 1 \mathrm{H}, \mathrm{H}-15\right), 4.35\left(\mathrm{~d},{ }^{2} J_{\mathrm{HH}}=9.8 \mathrm{~Hz}, 1 \mathrm{H}, \mathrm{H}-12\right), 4.31(\mathrm{~d}$, $\left.{ }^{2} J_{\mathrm{HH}}=9.8 \mathrm{~Hz}, 1 \mathrm{H}, \mathrm{H}-12\right), 3.26$ (s, 3H, H-16), 1.77 (s, 3H, H-14), 1.69 (s, 3H, H-14), 1.51 (s, 3H, H10), 1.51 (s, 3H, H-10) ppm. ${ }^{13} \mathbf{C}\left\{{ }^{1} \mathbf{H}\right\}-N M R ~\left(151 ~ M H z, ~ M e O D-d_{4}\right): \delta=157.7$ (C-8a), 156.7 (C-9a), 143.5 (C-4a), 137.9 (C-4b), 135.7 (C-3), 133.2 (C-2), 131.7 (C-6), 127.6 (C-8), 123.6 (C-7), 123.4 $(\mathrm{C}-5), 121.8\left(\mathrm{q}, \mathrm{CF}_{3},{ }^{1} J_{\mathrm{CF}}=318.4 \mathrm{~Hz}\right), 120.8(\mathrm{C}-1), 116.5(\mathrm{C}-4), 107.5(\mathrm{C}-11), 79.1(\mathrm{C}-12), 75.6(\mathrm{C}-$ 13), 65.8 (C-15), 48.4 (C-9), 44.2 (C-16), 27.1 (C-10), 27.0 (C-10), 23.2 (C-14), 18.8 (C-14) ppm. ${ }^{19} \mathbf{F}\left\{{ }^{1} \mathbf{H}\right\}$-NMR (565 MHz, MeOD- $\boldsymbol{d}_{4}$ ): $\delta=-80.09$ (s) ppm. IR (ATR, neat): $\tilde{v}=2965$ (w), $2927(\mathrm{w})$, 1623 (w), 1601 (w), 1475 (w), 1457 (w), 1436 (w), 1405 (w), 1388 (w), 1360 (w), 1251 (s), 1224 (s), 1190 (m), 1154 (s), 1086 (m), 1050 (m), 1028 (s), 968 (m), 918 (m), 878 (m), 820 (m), 755 (m), 676 (w) $\mathrm{cm}^{-1}$. MS (ESI): $\mathrm{m} / \mathrm{z}=397.8\left[\mathrm{C}_{22} \mathrm{H}_{25}{ }^{79} \mathrm{BrNO}\right]^{+}$. HR-MS (ESI): calculated for $\mathrm{C}_{22} \mathrm{H}_{25}{ }^{79} \mathrm{BrNO}^{+}$[M-OTf ]$^{+}: \mathrm{m} / \mathrm{z}=398.11140$, found: 398.11122 (Dev.: $-0.18 \mathrm{mu} ;-0.45 \mathrm{ppm}$ ).

\subsubsection{Attempted Preparation of (7-bromo-2-(4,4-dimethyl-4,5-dihydrooxazol-2-yl)-9,9- dimethyl-9H-fluoren-3-yl)methyl methanesulfonate (S3)}

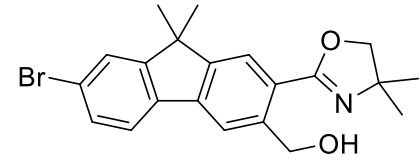

19

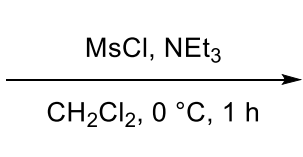

$\mathrm{CH}_{2} \mathrm{Cl}_{2}, 0{ }^{\circ} \mathrm{C}, 1 \mathrm{~h}$

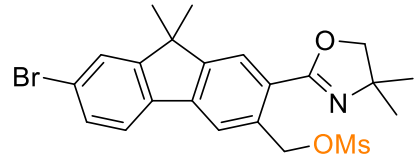

S3

A slightly modified literature procedure was used.[29] A heat gun-dried and nitrogen-flushed Schlenk tube, equipped with a magnetic stirring bar and a rubber septum, was charged with 19 (400 mg, $1.00 \mathrm{mmol}, 1.00 \mathrm{eq})$. The tube was flushed with nitrogen, anhydrous $\mathrm{CH}_{2} \mathrm{Cl}_{2}(5.00 \mathrm{~mL}, 0.2 \mathrm{M})$ was added and the colorless solution was cooled to $0{ }^{\circ} \mathrm{C}$. $\mathrm{NEt}_{3}(152 \mathrm{mg}, 209 \mu \mathrm{L}, 1.50 \mathrm{mmol}, 1.50 \mathrm{eq})$ and $\mathrm{MsCl}(160 \mathrm{mg}, 108 \mu \mathrm{L}, 1.40 \mathrm{mmol}, 1.40 \mathrm{eq})$ were added successively and the mixture was stirred at $0{ }^{\circ} \mathbf{C}$ for one hour. Despite complete conversion of $\mathbf{1 9}$, only traces of $\mathbf{S 3}$ were observed. 


\subsection{Preparation of Sterically Hindered Alkynes}

\subsubsection{Preparation of $(3 r, 5 r, 7 r)$-1-ethynyladamantane (31)}
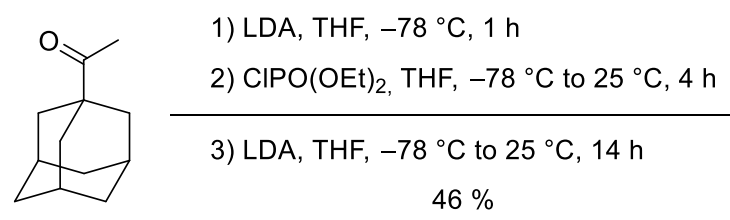

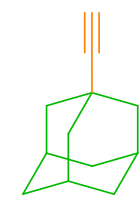

31

A modified literature procedure was used.[30] Initially a fresh LDA solution was prepared. Therefore, a heat gun-dried and nitrogen-flushed Schlenk flask, equipped with a magnetic stirring bar and a rubber septum, was charged with anhydrous THF $(16.7 \mathrm{~mL})$ and $N, N$-diisopropylamine $(1.69 \mathrm{~mL}, 12.0 \mathrm{mmol}$, $1.20 \mathrm{eq}$ ) was added. The solution was cooled to $-78^{\circ} \mathrm{C}$ and $n \operatorname{BuLi}(4.40 \mathrm{~mL}, 11.0 \mathrm{mmol}, 1.10 \mathrm{eq}, 2.50 \mathrm{M}$ in hexane) was added in one portion, whereupon the solution turned slightly yellow. After stirring for 30 minutes at the same temperature 1-((3r,5r,7r)-adamantan-1-yl)ethan-1-one $(1.78 \mathrm{~g}, 10.0 \mathrm{mmol}$, $1.00 \mathrm{eq})$, dissolved in anhydrous THF $(12.5 \mathrm{~mL})$, was added dropwise via syringe pump $(0.5 \mathrm{~mL} / \mathrm{min})$ to the LDA solution. After complete addition the solution was stirred at $-78{ }^{\circ} \mathrm{C}$ for an additional one hour and then diethyl chlorophosphate $(1.59 \mathrm{~mL}, 11.0 \mathrm{mmol}, 1.10 \mathrm{eq})$ was added via syringe pump $(0.5 \mathrm{~mL} / \mathrm{min})$, whereupon the mixture was stirred for one more hour under the same conditions. The cooling bath was removed and the mixture was stirred for three more hours while it was allowed to warm to $25^{\circ} \mathrm{C}$. In a separately heat gun-dried and nitrogen-flushed Schlenk flask, equipped with a magnetic stirring bar and a rubber septum, was prepared a second LDA solution. Therefore, $\mathrm{N}, \mathrm{N}$ diisopropylamine $(2.96 \mathrm{~mL}, 21.0 \mathrm{mmol}, 2.10 \mathrm{eq})$ was added to anhydrous THF $(25.0 \mathrm{~mL})$ and the solution was cooled to $-78^{\circ} \mathrm{C}$. $n \mathrm{BuLi}(8.00 \mathrm{~mL}, 20.0 \mathrm{mmol}, 2.00 \mathrm{eq}, 2.50 \mathrm{M}$ in hexane) was added in one portion and the slightly yellow solution was stirred for 30 minutes at the same temperature. The phosphate containing solution was added via syringe pump $(1.0 \mathrm{~mL} / \mathrm{min})$ to the second LDA solution at $-78{ }^{\circ} \mathrm{C}$ and stirred under these conditions for two hours. Then the mixture was allowed to warm slowly to $25^{\circ} \mathrm{C}$ and stirred for 12 hours in all. Saturated $\mathrm{NH}_{4} \mathrm{Cl}$ solution $(40 \mathrm{~mL})$ and water $(20 \mathrm{~mL})$ were added and the phases separated. The aqueous layer was extracted with ethyl acetate $(3 \times 40 \mathrm{~mL})$ and the combined organic layers were dried over anhydrous $\mathrm{Na}_{2} \mathrm{SO}_{4}$, filtered and the solvent was evaporated under reduced pressure. Purification by flash column chromatography $\left(\mathrm{SiO}_{2}, \mathrm{CH}\right)$ afforded $\mathbf{3 1}(735 \mathrm{mg}$, $4.59 \mathrm{mmol}, 46 \%)$ as a colorless solid.

$\mathbf{R}_{f}=0.74\left(\mathrm{SiO}_{2}, \mathrm{CH}\right.$, staining with $\left.\mathrm{KMnO}_{4}\right)$. Mp.: $79-81{ }^{\circ} \mathrm{C} .{ }^{1} \mathbf{H}-\mathrm{NMR}\left(360 \mathrm{MHz}, \mathbf{C D C l}_{3}\right): \delta=2.10$ (s, 1H), 1.95 (br. s, 3H), $1.88\left(\mathrm{~d},{ }^{3} \mathrm{~J}_{\mathrm{HH}}=2.8 \mathrm{~Hz}, 6 \mathrm{H}\right), 1.68\left(\mathrm{t},{ }^{3} J_{\mathrm{HH}}=3.1 \mathrm{~Hz}, 6 \mathrm{H}\right) \mathrm{ppm} .{ }^{13} \mathbf{C}\left\{{ }^{1} \mathbf{H}\right\}-\mathbf{N M R}$ (91 MHz, $\left.\mathbf{C D C l}_{3}\right): \delta=93.2,66.7,42.9(3 \mathrm{x}), 36.4(3 \mathrm{x}), 29.5,28.0(3 \mathrm{x}) \mathrm{ppm}$. IR (ATR, neat): $\tilde{v}=3304$ (m), 2900 (s), 2849 (s), 2657 (w), 2104 (w), 1450 (s), 1356 (w), 1343 (w), 1314 (w), 1227 (m), 1182 


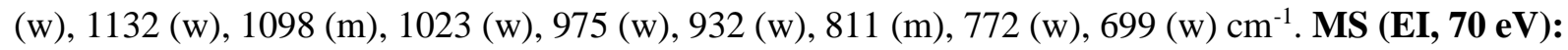
$\mathrm{m} / \mathrm{z}=160.1[\mathrm{M}]^{+}$. The analytical data are in accordance with the literature.[30]

\subsubsection{Preparation of 1,3-di-tert-butyl-5-ethynylbenzene (32)}

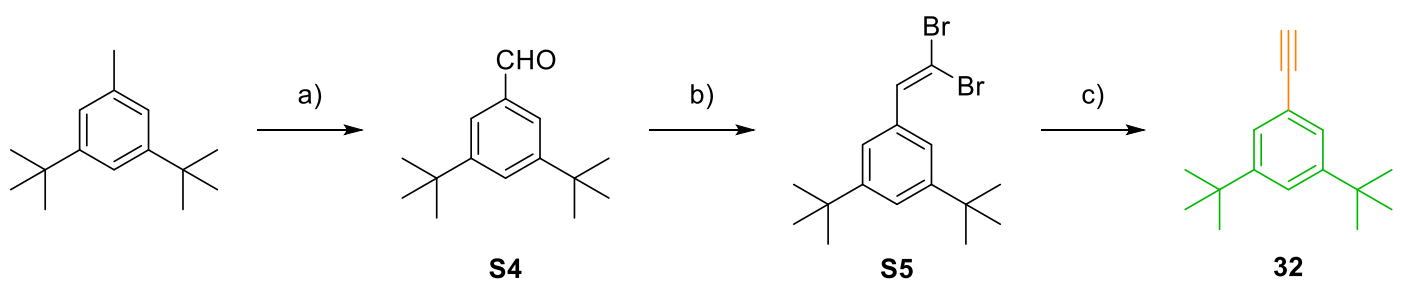

Reaction conditions: a) 1) NBS, AIBN, $\mathrm{PhH}, 90{ }^{\circ} \mathrm{C}, 16 \mathrm{~h}$; 2) $\left(\mathrm{CH}_{2}\right)_{6} \mathrm{~N}_{4}, \mathrm{EtOH}: \mathrm{H}_{2} \mathrm{O} 1: 1 \mathrm{v}: \mathrm{v}, 90^{\circ} \mathrm{C} 4 \mathrm{~h}$; (3) conc. $\mathrm{HCl}, 90{ }^{\circ} \mathrm{C}$, $0.5 \mathrm{~h}, 80 \%$; b) $\mathrm{PPh}_{3}, \mathrm{Zn}, \mathrm{CBr}_{4}, \mathrm{CH}_{2} \mathrm{Cl}_{2}, 0{ }^{\circ} \mathrm{C}$ to $25^{\circ} \mathrm{C}, 3 \mathrm{~h}, 96 \%$; c) $n \mathrm{BuLi}, \mathrm{THF},-78^{\circ} \mathrm{C}$ to $25{ }^{\circ} \mathrm{C}, 5 \mathrm{~h}, 89 \%$.

\subsubsection{3,5-Di-tert-butylbenzaldehyde (S4)}

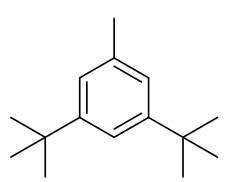

1) NBS, AIBN, $\mathrm{PhH}, 90^{\circ} \mathrm{C}, 16 \mathrm{~h}$

2) $\left(\mathrm{CH}_{2}\right)_{6} \mathrm{~N}_{4}$, EtOH: $\mathrm{H}_{2} \mathrm{O} 1: 1 \mathrm{v:v}, 90^{\circ} \mathrm{C} 4 \mathrm{~h}$

3) conc. $\mathrm{HCl}, 90^{\circ} \mathrm{C}, 0.5 \mathrm{~h}$

$80 \%$

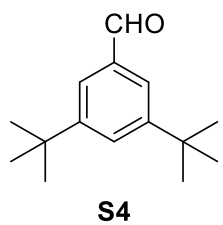

A modified literature procedure was used.[31] 1,3-Di-tert-butyl-5-methylbenzene (3.07 g, $15.0 \mathrm{mmol}$, $1.00 \mathrm{eq})$ was dissolved in benzene $(15.0 \mathrm{~mL}, 1.0 \mathrm{M})$ and NBS $(1.50 \mathrm{~g}, 22.5 \mathrm{mmol}, 1.50 \mathrm{eq})$ and AIBN (123 mg, $750 \mu \mathrm{mol}, 0.05 \mathrm{eq}$ ) were added. The mixture was heated to $100{ }^{\circ} \mathrm{C}$ (oil bath temperature) and stirred for 16 hours at this temperature. After cooling to $25^{\circ} \mathrm{C}$, the mixture was filtered through Celite ${ }^{\circledR}$ and washed with ethyl acetate $(\sim 50 \mathrm{~mL})$. The solution was concentrated under reduced pressure to afford a brown oil, which was used without further purification. The crude product was dissolved in EtOH: $\mathrm{H}_{2} \mathrm{O}$ $(15 \mathrm{~mL} 1: 1 \mathrm{v}: \mathrm{v})$ and hexamethylenetetramine $(6.31 \mathrm{~g}, 45.0 \mathrm{mmol}, 3.00 \mathrm{eq})$ was added in one portion. Heating for four hours at $100{ }^{\circ} \mathrm{C}$ (oil bath temperature) was followed by the addition of concentrated $\mathrm{HCl}$ and heating was continued for another 30 minutes. Ethanol was removed under reduced pressure by rotary evaporation and water $(35 \mathrm{~mL})$ and ethyl acetate $(50 \mathrm{~mL})$ were added. The phases were separated and the aqueous phase was extracted with ethyl acetate $(3 \times 50 \mathrm{~mL})$. The combined organic phases were dried over anhydrous $\mathrm{Na}_{2} \mathrm{SO}_{4}$, filtered and concentrated under reduced pressure. Purification by flash column chromatography $\left(\mathrm{SiO}_{2}, \mathrm{CH}: \mathrm{EE}\right.$ 50:1 v:v) afforded $\mathbf{S 4}$ (2.61 g, $12.0 \mathrm{mmol}$, $80 \%$ ) as a colorless oil, which slowly solidified upon standing at $25^{\circ} \mathrm{C}$.

$\mathbf{R}_{f}=0.09\left(\mathrm{SiO}_{2}, \mathrm{CH}\right) . \mathbf{R}_{\boldsymbol{f}}=0.26\left(\mathrm{SiO}_{2}, \mathrm{CH}: \mathrm{EE} 50: 1 \mathrm{v}: \mathrm{v}\right)$. Mp.: $83-85^{\circ} \mathrm{C} .{ }^{1} \mathbf{H}-\mathbf{N M R}\left(\mathbf{3 6 0} \mathbf{~ M H z}, \mathbf{C D C l}_{3}\right)$ : $\delta=10.01(\mathrm{~s}, 1 \mathrm{H}), 7.76-7.69(\mathrm{~m}, 3 \mathrm{H}), 1.37$ (s, 18H) ppm. ${ }^{13} \mathbf{C}\left\{{ }^{1} \mathbf{H}\right\}-\mathbf{N M R}\left(\mathbf{9 1} \mathbf{~ M H z}, \mathbf{C D C l}_{3}\right): \delta=193.4$, 152.0 (2x), 136.3, 129.0, 124.3 (2x), 35.1 (2x), 31.5 (6x) ppm. IR (ATR, neat): $\tilde{v}=2960$ (m), 2905 (w), 2868 (w), 2817 (w), 2734 (w), 1686 (s), 1591 (m), 1474 (m), 1463 (m), 1388 (m), 1363 (m), 1325 
(w), $1305(\mathrm{w}), 1278(\mathrm{w}), 1250(\mathrm{~m}), 1189(\mathrm{~s}), 1127(\mathrm{w}), 1011(\mathrm{w}), 948(\mathrm{w}), 900(\mathrm{w}), 882(\mathrm{~m}), 818(\mathrm{~m})$, $768(\mathrm{w}), 706(\mathrm{~s}), 699(\mathrm{~s}) \mathrm{cm}^{-1}$. MS (EI, 70 eV): $\mathrm{m} / \mathrm{z}=218.1[\mathrm{M}]^{+.}$. The analytical data are in accordance with the literature.[32]

\subsubsection{1,3-Di-tert-butyl-5-(2,2-dibromovinyl)benzene (S5)}<smiles>CC(C)(C)c1cc(C=O)cc(C(C)(C)C)c1</smiles>

S4

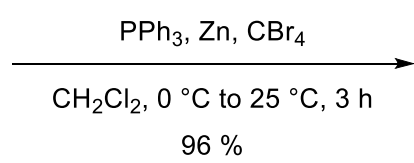

$96 \%$

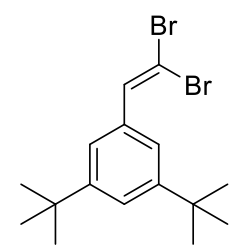

S5

A modified literature procedure was used.[33] A heat gun-dried and nitrogen-flushed Schlenk flask, equipped with a magnetic stirring bar and a rubber septum, was charged with $\mathrm{PPh}_{3}(4.72 \mathrm{~g}, 18.0 \mathrm{mmol}$, $2.00 \mathrm{eq}$ ) and anhydrous $\mathrm{CH}_{2} \mathrm{Cl}_{2}(45 \mathrm{~mL})$ was added. Zinc dust (particle $<10 \mu \mathrm{m}$ ) was added in one portion and the suspension was cooled to $0{ }^{\circ} \mathrm{C}$. $\mathrm{CBr}_{4}(5.97 \mathrm{~g}, 18.0 \mathrm{mmol}, 2.00 \mathrm{eq})$ was added in one portion whereupon the suspension turned dirty green. The mixture was allowed to warm slowly to ambient temperature and stirred for 24 hours, while the color turned deep red. S4 (1.97 g, 9.00 mmol, $1.00 \mathrm{eq})$ was dissolved in anhydrous $\mathrm{CH}_{2} \mathrm{Cl}_{2}(15 \mathrm{~mL})$ and added via syringe pump $(1.0 \mathrm{~mL} / \mathrm{min})$ to the ice cooled $\left(0{ }^{\circ} \mathrm{C}\right)$ mixture. Stirring was continued for three more hours while the mixture was allowed to warm to $25{ }^{\circ} \mathrm{C}$. Filtration through a short plug of Celite ${ }^{\circledR}$ and washing with $\mathrm{CH}_{2} \mathrm{Cl}_{2}(150 \mathrm{~mL})$ was followed by removing of the solvent under reduced pressure. Purification by flash column chromatography $\left(\mathrm{SiO}_{2}, \mathrm{CH}\right)$ afforded $\mathbf{S 5}(3.23 \mathrm{~g}, 8.63 \mathrm{mmol}, 96 \%)$ as a colorless oil which slowly solidified upon standing at $25^{\circ} \mathrm{C}$.

$\mathbf{R}_{f}=0.78\left(\mathrm{SiO}_{2}, \mathrm{CH}\right)$. Mp.: $41-44{ }^{\circ} \mathrm{C} .{ }^{1} \mathbf{H}-\mathbf{N M R}\left(\mathbf{3 6 0} \mathbf{M H z}, \mathbf{C D C l}_{3}\right): \delta=7.52$ (br. s, $\left.1 \mathrm{H}\right), 7.43-7.37$ (m, 3H), 1.34 (s, 18H) ppm. ${ }^{13} \mathbf{C}\left\{{ }^{1} \mathbf{H}\right\}-\mathbf{N M R}$ (91 MHz, $\left.\mathbf{C D C l}_{3}\right): \delta=150.9(2 \mathrm{x}), 137.9,134.5,122.9$ (2x), 122.8, 88.7, 35.0 (2x), 31.5 (6x) ppm. IR (ATR, neat): $\tilde{v}=3308$ (w), 2961 (s), 2903 (s), 2853 (m), 2733 (w), 2105 (w), 1687 (s), 1593 (m), 1475 (m), 1451 (m), 1389 (m), 1363 (s), 1305 (w), 1278 (w), 1249 (m), 1189 (s), 1127 (w), 1098 (w), 1011 (w), 949 (w), 900 (m), 882 (s), 827 (m), 817 (m), 799 (m), 767 (w), $705(\mathrm{~s}) \mathrm{cm}^{-1}$. MS (EI, $\left.70 \mathrm{eV}\right): \mathrm{m} / \mathrm{z}=371.9\left[\mathrm{C}_{16} \mathrm{H}_{22}{ }^{79} \mathrm{Br}_{2}\right]^{+}$. HR-MS (EI, $70 \mathrm{eV}$ ): calculated for $\mathrm{C}_{16} \mathrm{H}_{22}{ }^{79} \mathrm{Br}_{2}{ }^{+\cdot}[\mathrm{M}]^{+*}: \mathrm{m} / \mathrm{z}=372.00883$, found: 372.00842 (Dev.: $0.41 \mathrm{mu} ; 1.10 \mathrm{ppm}$ ). The analytical data are in accordance with the literature.[33] 


\subsubsection{1,3-Di-tert-butyl-5-ethynylbenzene (32)}

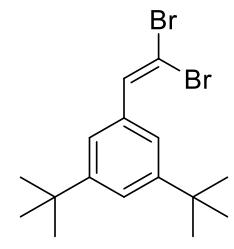

S5

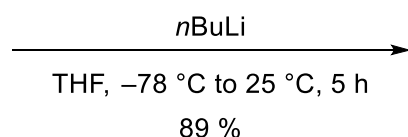

$89 \%$

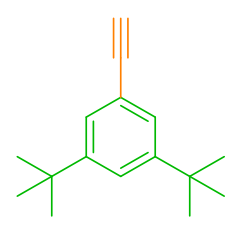

32

A modified literature procedure was used.[33] A heat gun-dried and nitrogen-flushed Schlenk flask, equipped with a magnetic stirring bar and a rubber septum, was charged with $\mathbf{S 5}$ (1.87 g, $5.00 \mathrm{mmol}$, $1.00 \mathrm{eq})$ and dissolved in anhydrous THF $(12.5 \mathrm{~mL}, 0.4 \mathrm{M})$. The solution was cooled to $-78{ }^{\circ} \mathrm{C}$ and $n \operatorname{BuLi}(6.00 \mathrm{~mL}, 15.0 \mathrm{mmol}, 3.00 \mathrm{eq}, 2.50 \mathrm{M}$ in hexane) was added via syringe pump $(1.0 \mathrm{~mL} / \mathrm{min})$. Stirring was continued for one hour at $-78{ }^{\circ} \mathrm{C}$, then the cooling bath was removed while it was allowed to warm slowly to $25^{\circ} \mathrm{C}$ and stirred for four hours in all. Cooling to $0{ }^{\circ} \mathrm{C}$ was followed by the dropwise addition of saturated $\mathrm{NH}_{4} \mathrm{Cl}$ solution $(10 \mathrm{~mL})$. Water $(10 \mathrm{~mL})$ and $\mathrm{CH}_{2} \mathrm{Cl}_{2}(10 \mathrm{~mL})$ were added and the phases separated. The aqueous phase was extracted with $\mathrm{CH}_{2} \mathrm{Cl}_{2}(3 \times 20 \mathrm{~mL})$, the combined organic phases dried over anhydrous $\mathrm{Na}_{2} \mathrm{SO}_{4}$, filtered and concentrated under reduced pressure. Purification by flash column chromatography $\left(\mathrm{SiO}_{2}, \mathrm{CH}\right)$ provided $32(949 \mathrm{mg}, 4.43 \mathrm{mmol}, 89 \%)$ as a colorless solid.

$\mathbf{R}_{f}=0.66\left(\mathrm{SiO}_{2}, \mathrm{CH}\right)$. Mp.: $83-85^{\circ} \mathrm{C} .{ }^{1} \mathbf{H}-\mathbf{N M R}\left(360 \mathrm{MHz}, \mathbf{C D C l}_{3}\right): \delta=7.45-7.38(\mathrm{~m}, 1 \mathrm{H}), 7.38-$ $7.32(\mathrm{~m}, 2 \mathrm{H}), 3.03$ (s, 1H), 1.31 (s, 18H) ppm. ${ }^{13} \mathbf{C}\left\{{ }^{1} \mathbf{H}\right\}-\mathbf{N M R}$ (91 $\left.\mathbf{M H z}, \mathbf{C D C l}_{3}\right): \delta=151.0(2 \mathrm{x}), 126.5$ (2x), 123.4, 121.1, 85.0, 75.9, 34.9 (2x), 31.4 (6x) ppm. IR (ATR, neat): $\tilde{v}=3272$ (m), 2951 (s), 2903 (m), 2868 (m), 1776 (w), 1591 (m), 1477 (m), 1460 (m), 1422 (m), 1392 (w), 1364 (s), 1316 (w), 1248 (m), 1192 (m), 1137 (w), 1025 (w), 935 (w), 914 (w), 900 (m), 883 (s), 710 (s), 673 (s) cm c $^{-1}$ MS (EI, $70 \mathrm{eV}): \mathrm{m} / \mathrm{z}=214.1[\mathrm{M}]^{+}$. HR-MS (EI, 70 eV): calculated for $\mathrm{C}_{16} \mathrm{H}_{22}{ }^{+\cdot}[\mathrm{M}]^{+\cdot}: \mathrm{m} / \mathrm{z}=214.17215$, found: 214.17143 (Dev.: $0.72 \mathrm{mu} ; 3.36 \mathrm{ppm}$ ). The analytical data are in accordance with the literature.[33] 


\subsection{Copper-Catalyzed Azide-Alkyne Cycloadditions (CuAAC)}

\subsubsection{General Procedure for the CuAACs (GP1)}

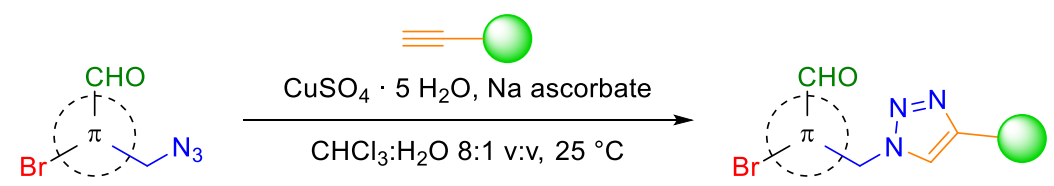

A modified literature procedure was used.[34] In a screw-cap vial azide (100 $\mu \mathrm{mol}, 1.00 \mathrm{eq})$ and terminal alkyne (105 $\mu \mathrm{mol}, 1.05 \mathrm{eq})$ were dissolved in $\mathrm{CHCl}_{3}(1.00 \mathrm{~mL}, 0.1 \mathrm{M}) . \mathrm{CuSO}_{4} \cdot 5 \mathrm{H}_{2} \mathrm{O}(2.4 \mathrm{mg}, 10 \mu \mathrm{mol}$, $0.10 \mathrm{eq})$ and sodium ascorbate $(9.9 \mathrm{mg}, 50 \mu \mathrm{mol}, 0.50 \mathrm{eq})$ were suspended separately in water $(125 \mu \mathrm{L})$ and added to the reaction vial. The heterogeneous mixture was stirred vigorously at $25{ }^{\circ} \mathrm{C}$ for the indicated time and directly purified by flash column chromatography.

\subsubsection{4-Bromo-2-((4-octyl-1H-1,2,3-triazol-1-yl)methyl)benzaldehyde (33)}

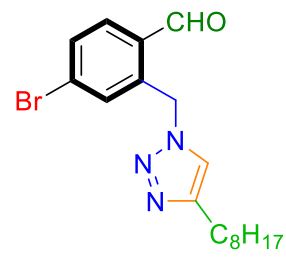

33

According to GP1 the reaction of $\mathbf{3}(24.0 \mathrm{mg}, 100 \mu \mathrm{mol}, 1.00 \mathrm{eq})$ and 1-decyne (19.0 $\mu \mathrm{L}, 105 \mu \mathrm{mol}$, $1.05 \mathrm{eq})$ provided after 24 hours reaction time and subsequent flash column chromatography $\left(\mathrm{SiO}_{2}\right.$, CH:EE 3:1 v:v) $32(35.5 \mathrm{mg}, 93.8 \mu \mathrm{mol}, 94 \%)$ as a colorless solid.

$\mathbf{R}_{f}=0.33\left(\mathrm{SiO}_{2}, \mathrm{CH}: \mathrm{EE} 3: 1 \mathrm{v}: \mathrm{v}\right)$. Mp.: $82-84{ }^{\circ} \mathrm{C} .{ }^{1} \mathbf{H}-\mathbf{N M R}\left(\mathbf{3 6 0} \mathbf{M H z}, \mathbf{C D C l}_{3}\right): \delta=10.08\left(\mathrm{~d},{ }^{4} \boldsymbol{J}_{\mathrm{HH}}=\right.$ $0.6 \mathrm{~Hz}, 1 \mathrm{H}), 7.72-7.67(\mathrm{~m}, 2 \mathrm{H}), 7.39(\mathrm{~s}, 1 \mathrm{H}), 7.22-7.16(\mathrm{~m}, 1 \mathrm{H}), 5.94(\mathrm{~s}, 2 \mathrm{H}), 2.71\left(\mathrm{t},{ }^{3} J_{\mathrm{HH}}=7.6 \mathrm{~Hz}\right.$, $2 \mathrm{H}), 1.66\left(\mathrm{p},{ }^{3} J_{\mathrm{HH}}=7.9 \mathrm{~Hz}, 2 \mathrm{H}\right), 1.39-1.20(\mathrm{~m}, 10 \mathrm{H}), 0.86\left(\mathrm{t},{ }^{3} J_{\mathrm{HH}}=7.2 \mathrm{~Hz}, 3 \mathrm{H}\right) \mathrm{ppm} .{ }^{13} \mathbf{C}\left\{{ }^{1} \mathbf{H}\right\}-\mathbf{N M R}$ (91 MHz, $\left.\mathbf{C D C l}_{3}\right): \delta=192.6,149.1,138.6,136.7,132.9,132.3,131.8,130.1,121.7,50.2,32.0,29.5$, 29.4, 29.4, 29.3, 25.8, 22.8, 14.2 ppm. IR (ATR, neat): $\tilde{v}=3056$ (w), 2950 (w), 2921 (m), 2850 (m), 2782 (w), 1686 (s), 1583 (s), 1562 (m), 1466 (w), 1435 (m), 1334 (m), 1288 (w), 1210 (m), 1196 (m), 1174 (m), 1141 (m), 1097 (m), 1079 (m), 1055 (m), 1033 (w), 866 (s), 834 (m), 803 (s), 721 (m), 670 (w) $\mathrm{cm}^{-1}$. MS (APCI): $\mathrm{m} / \mathrm{z}=378.1\left[\mathrm{C}_{18} \mathrm{H}_{24}{ }^{79} \mathrm{BrN}_{3} \mathrm{O}+\mathrm{H}\right]^{+}$. HR-MS (EI, $70 \mathrm{eV}$ ): calculated for $\mathrm{C}_{18} \mathrm{H}_{24}{ }^{79} \mathrm{BrN}_{3} \mathrm{O}^{+\cdot}[\mathrm{M}]^{+*}: \mathrm{m} / \mathrm{z}=377.10973$, found: 377.10924 (Dev.: $-0.49 \mathrm{mu} ;-1.29 \mathrm{ppm}$ ); calculated for $\mathrm{C}_{18} \mathrm{H}_{24}{ }^{81} \mathrm{BrN}_{3} \mathrm{O}^{+\cdot}[\mathrm{M}]^{+\cdot}: \mathrm{m} / \mathrm{z}=379.10768$, found: 379.10780 (Dev.: $0.12 \mathrm{mu} ; 0.32 \mathrm{ppm}$ ). 


\subsubsection{2-((4-((1s,3s)-Adamantan-1-yl)-1H-1,2,3-triazol-1-yl)methyl)-4- bromobenzaldehyde (34)}

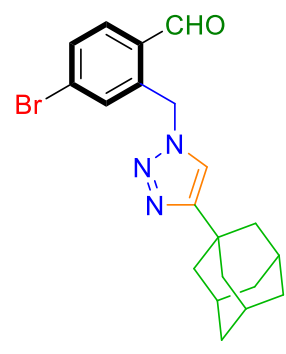

34

According to GP1 the reaction of $\mathbf{3}(24.0 \mathrm{mg}, 100 \mu \mathrm{mol}, 1.00 \mathrm{eq})$ and $\mathbf{3 1}(16.8 \mathrm{mg}, 105 \mu \mathrm{mol}, 1.05 \mathrm{eq})$ provided after three days reaction time and subsequent flash column chromatography ( $\mathrm{SiO}_{2}, \mathrm{CH}: \mathrm{EE} 4: 1$ v:v) 34 (38.9 mg, $97.2 \mu \mathrm{mol}, 97 \%)$ as a colorless solid.

$\mathbf{R}_{f}=0.23\left(\mathrm{SiO}_{2}, \mathrm{CH}: \mathrm{EE} 4: 1 \mathrm{v}: \mathrm{v}\right) . \mathbf{M p} .: 131-133{ }^{\circ} \mathrm{C} .{ }^{1} \mathbf{H}-\mathbf{N M R}\left(\mathbf{3 6 0} \mathbf{M H z}, \mathbf{C D C l}_{\mathbf{3}}\right): \delta=10.08(\mathrm{~s}, 1 \mathrm{H})$, $7.69\left(\mathrm{~d},{ }^{4} J_{\mathrm{HH}}=1.6 \mathrm{~Hz}, 2 \mathrm{H}\right), 7.34(\mathrm{~s}, 1 \mathrm{H}), 7.24-7.19(\mathrm{~m}, 1 \mathrm{H}), 5.93(\mathrm{~s}, 2 \mathrm{H}), 2.08-2.02(\mathrm{~m}, 3 \mathrm{H}), 1.96$ $\left(\mathrm{d},{ }^{3} J_{\mathrm{HH}}=2.9 \mathrm{~Hz}, 6 \mathrm{H}\right), 1.82-1.71(\mathrm{~m}, 6 \mathrm{H}) \mathrm{ppm} .{ }^{13} \mathbf{C}\left\{{ }^{1} \mathbf{H}\right\}-\mathbf{N M R}\left(\mathbf{9 1} \mathbf{M H z}, \mathbf{C D C l}_{3}\right): \delta=192.6,158.5$, 138.6, 136.6, 133.1, 132.3, 131.8, 130.0, 119.5, 50.1, 42.6 (3x), 36.8 (3x), 32.8, 28.5 (3x) ppm. IR (ATR, neat): $\tilde{v}=2906$ (m), 2848 (m), 1694 (s), 1587 (m), 1558 (m), 1479 (w), 1456 (m), 1413 (w), 1385 (w), 1340 (m), 1309 (m), 1290 (m), 1212 (m), 1186 (m), 1170 (m), 1146 (m), 1077 (m), 1047 (m), 1001 (m), 976 (w), 888 (s), 852 (w), 804 (s), 754 (m), 723 (m), 701 (m), 683 (m), 661 (m) cm c $^{-1}$ MS (APCI): $\mathrm{m} / \mathrm{z}=400.1\left[\mathrm{C}_{20} \mathrm{H}_{22}{ }^{79} \mathrm{BrN}_{3} \mathrm{O}+\mathrm{H}\right]^{+}$. HR-MS (ESI): calculated for $\mathrm{C}_{20} \mathrm{H}_{23}{ }^{79} \mathrm{BrN}_{3} \mathrm{O}^{+}[\mathrm{M}+\mathrm{H}]^{+}: \mathrm{m} / \mathrm{z}$ $=400.10190$, found: 400.10175 (Dev.: $-0.15 \mathrm{mu} ;-0.37 \mathrm{ppm}$ ).

\subsubsection{4-Bromo-2-((4-phenyl-1H-1,2,3-triazol-1-yl)methyl)benzaldehyde (35)}

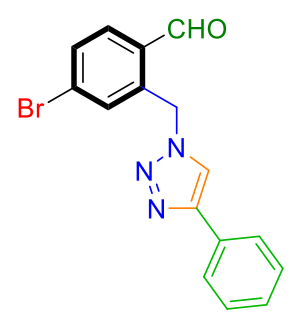

35

According to GP1 the reaction of $3(24.0 \mathrm{mg}, 100 \mu \mathrm{mol}, 1.00 \mathrm{eq})$ and phenylacetylene $(11.5 \mu \mathrm{L}$, $105 \mu \mathrm{mol}, 1.05 \mathrm{eq})$ provided after 24 hours reaction time and subsequent flash column chromatography ( $\left.\mathrm{SiO}_{2}, \mathrm{CH}: \mathrm{EE} 3: 1 \mathrm{v}: \mathrm{v}\right) 35$ (33.8 mg, $\left.98.7 \mu \mathrm{mol}, 99 \%\right)$ as a colorless solid.

$\mathbf{R}_{f}=0.27\left(\mathrm{SiO}_{2}, \mathrm{CH}: \mathrm{EE} 3: 1 \mathrm{v}: \mathrm{v}\right)$. Mp.: $157-159{ }^{\circ} \mathrm{C} .{ }^{1} \mathbf{H}-\mathrm{NMR}\left(\mathbf{3 6 0} \mathbf{M H z}, \mathbf{C D C l}_{\mathbf{3}}\right): \delta=10.08\left(\mathrm{~d},{ }^{4} \mathrm{~J}_{\mathrm{HH}}\right.$ $=0.6 \mathrm{~Hz}, 1 \mathrm{H}), 7.92(\mathrm{~s}, 1 \mathrm{H}), 7.87-7.79(\mathrm{~m}, 2 \mathrm{H}), 7.72-7.70(\mathrm{~m}, 2 \mathrm{H}), 7.44-7.38(\mathrm{~m}, 2 \mathrm{H}), 7.37-7.35$ (m, 1H), $7.35-7.30(\mathrm{~m}, 1 \mathrm{H}), 6.02(\mathrm{~s}, 2 \mathrm{H}) \mathrm{ppm} .{ }^{13} \mathbf{C}\left\{{ }^{1} \mathbf{H}\right\}-\mathbf{N M R}\left(\mathbf{9 1} \mathbf{M H z}, \mathbf{C D C l}_{3}\right): \delta=192.7,148.1$, 
138.0, 136.9, 133.3, 132.5, 131.8, 130.5, 130.1, 129.0 (2x), 128.4, 125.8 (2x), 120.7, 50.4 ppm. IR (ATR, neat): $\tilde{v}=3082(\mathrm{w}), 2876(\mathrm{w}), 2777(\mathrm{w}), 1686(\mathrm{~s}), 1582(\mathrm{~m}), 1563(\mathrm{~m}), 1466(\mathrm{~m}), 1433(\mathrm{~m})$, 1400 (w), 1349 (w), 1288 (w), 1214 (m), 1202 (m), 1172 (m), 1099 (w), 1084 (m), 1047 (m), 1029 (w), 978 (m), 915 (w), 865 (m), 833 (m), 804 (m), 781 (m), 765 (s), 714 (m), 692 (s), 671 (m), 657 (m) $\mathrm{cm}^{-1}$. MS (APCI): $\mathrm{m} / \mathrm{z}=342.0\left[\mathrm{C}_{16} \mathrm{H}_{12}{ }^{79} \mathrm{BrN}_{3} \mathrm{O}+\mathrm{H}\right]^{+}$. HR-MS (EI, $70 \mathrm{eV}$ ): calculated for $\mathrm{C}_{16} \mathrm{H}_{12}{ }^{79} \mathrm{BrN}_{3} \mathrm{O}^{+\cdot}[\mathrm{M}]^{+\cdot}: \mathrm{m} / \mathrm{z}=341.01583$, found: 341.01570 (Dev.: $-0.13 \mathrm{mu} ;-0.37 \mathrm{ppm}$ ).

\subsubsection{4-Bromo-2-((4-(3,5-di-tert-butylphenyl)-1H-1,2,3-triazol-1- yl)methyl)benzaldehyde (36)}

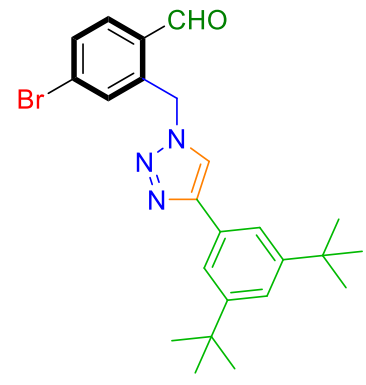

36

According to GP1 the reaction of $\mathbf{3}(24.0 \mathrm{mg}, 100 \mu \mathrm{mol}, 1.00 \mathrm{eq})$ and $\mathbf{3 2}(22.5 \mathrm{mg}, 105 \mu \mathrm{mol}, 1.05 \mathrm{eq})$ provided after three days reaction time and subsequent flash column chromatography $\left(\mathrm{SiO}_{2}, \mathrm{CH}: \mathrm{EE} 4: 1\right.$ v:v) $36(43.6 \mathrm{mg}, 96.0 \mu \mathrm{mol}, 96 \%)$ as a colorless solid.

$\mathbf{R}_{f}=0.31\left(\mathrm{SiO}_{2}, \mathrm{CH}: \mathrm{EE} 4: 1 \mathrm{v}: \mathrm{v}\right) . \mathbf{M p . :} 132-134{ }^{\circ} \mathrm{C} .{ }^{\mathbf{1}} \mathbf{H}-\mathbf{N M R}\left(\mathbf{3 6 0} \mathbf{M H z}, \mathbf{C D C l}_{\mathbf{3}}\right): \delta=10.10(\mathrm{~s}, 1 \mathrm{H})$, $7.91(\mathrm{~s}, 1 \mathrm{H}), 7.71\left(\mathrm{~d},{ }^{5} J_{\mathrm{HH}}=1.0 \mathrm{~Hz}, 2 \mathrm{H}\right), 7.69\left(\mathrm{~d},{ }^{4} J_{\mathrm{HH}}=1.8 \mathrm{~Hz}, 2 \mathrm{H}\right), 7.43\left(\mathrm{t},{ }^{4} J_{\mathrm{HH}}=1.8 \mathrm{~Hz}, 1 \mathrm{H}\right), 7.31$ (s, 1H), 6.06 (s, 2H), 1.37 (s, 18H) ppm. ${ }^{13} \mathbf{C}\left\{{ }^{1} \mathbf{H}\right\}-N M R\left(\mathbf{9 1} \mathbf{M H z}, \mathbf{C D C l}_{3}\right): \delta=192.7,151.5$ (2x), 149.1, 138.3, 136.8, 133.0, 132.4, 131.8, 130.2, 129.6, 122.7, 120.6 (2x), 120.3, 50.4, 35.1 (2x), $31.6(6 x)$ ppm. IR (ATR, neat): $\tilde{v}=2962$ (m), 2903 (m), 2847 (w), 1698 (s), 1590 (m), 1562 (s), 1447 (m), 1419 (m), 1393 (m), 1361 (m), 1343 (m), 1287 (m), 1249 (m), 1225 (m), 1201 (m), 1183 (m), 1097 (m), 1080 (m), 1064 (m), 1051 (s), 1001 (w), 968 (m), 902 (s), 879 (s), 848 (m), 826 (s), 816 (s), 785 (s), 768 (s), 727 (s), $718(\mathrm{~s}), 709$ (s), $685(\mathrm{~m}), 673(\mathrm{~m}), 660(\mathrm{~m}) \mathrm{cm}^{-1}$. MS (APCI): m/z = 454.0 $\left[\mathrm{C}_{24} \mathrm{H}_{28}{ }^{79} \mathrm{BrN}_{3} \mathrm{O}+\mathrm{H}\right]^{+}$. HR-MS (ESI): calculated for $\mathrm{C}_{24} \mathrm{H}_{29}{ }^{79} \mathrm{BrN}_{3} \mathrm{O}^{+}[\mathrm{M}+\mathrm{H}]^{+}: \mathrm{m} / \mathrm{z}=454.14885$, found: 454.14826 (Dev.: $-0.59 \mathrm{mu} ;-1.30 \mathrm{ppm})$.

\subsubsection{2-Bromo-4-((4-octyl-1H-1,2,3-triazol-1-yl)methyl)benzaldehyde (37)}

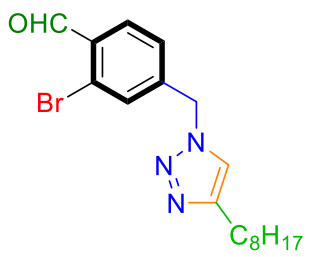

37 
According to GP1 the reaction of $4(24.0 \mathrm{mg}, 100 \mu \mathrm{mol}, 1.00 \mathrm{eq})$ and 1-decyne (19.0 $\mu \mathrm{L}, 105 \mu \mathrm{mol}$, $1.05 \mathrm{eq})$ provided after 24 hours reaction time and subsequent flash column chromatography $\left(\mathrm{SiO}_{2}\right.$, CH:EE 3:1 v:v) 37 (34.7 mg, $91.7 \mu \mathrm{mol}, 92 \%)$ as a colorless solid.

$\mathbf{R}_{f}=0.14\left(\mathrm{SiO}_{2}, \mathrm{CH}: \mathrm{EE} 3: 1 \mathrm{v}: \mathrm{v}\right)$. Mp.: $75-77{ }^{\circ} \mathrm{C} .{ }^{1} \mathbf{H}-\mathbf{N M R}\left(\mathbf{3 6 0} \mathbf{M H z}, \mathbf{C D C l}_{3}\right): \delta=10.30\left(\mathrm{~d},{ }^{4} \boldsymbol{J}_{\mathrm{HH}}=\right.$ $0.8 \mathrm{~Hz}, 1 \mathrm{H}), 7.87\left(\mathrm{~d},{ }^{3} J_{\mathrm{HH}}=8.0 \mathrm{~Hz}, 1 \mathrm{H}\right), 7.50-7.44(\mathrm{~m}, 1 \mathrm{H}), 7.30-7.21(\mathrm{~m}, 2 \mathrm{H}), 5.53(\mathrm{~s}, 2 \mathrm{H}), 2.70(\mathrm{t}$, $\left.{ }^{3} J_{\mathrm{HH}}=7.7 \mathrm{~Hz}, 2 \mathrm{H}\right), 1.64\left(\mathrm{p},{ }^{3} J_{\mathrm{HH}}=7.9 \mathrm{~Hz}, 2 \mathrm{H}\right), 1.36-1.19(\mathrm{~m}, 10 \mathrm{H}), 0.84\left(\mathrm{t},{ }^{3} J_{\mathrm{HH}}=6.8 \mathrm{~Hz}, 3 \mathrm{H}\right) \mathrm{ppm}$. ${ }^{13} \mathbf{C}\left\{{ }^{1} \mathbf{H}\right\}$-NMR (91 MHz, $\left.\mathbf{C D C l}_{3}\right): \delta=191.1,149.6,142.9,133.5,132.8,130.5,127.6,127.1,120.9$, 52.8, 31.9, 29.4, 29.4, 29.3, 29.3, 25.8, 22.7, 14.2 ppm. IR (ATR, neat): $\tilde{v}=3061$ (w), 2951 (w), 2918 (m), 2848 (m), 1687 (s), 1600 (m), 1558 (m), 1466 (m), 1432 (m), 1408 (m), 1389 (m), 1349 (m), 1291 (w), 1260 (m), 1202 (s), 1137 (m), 1079 (w), 1052 (m), 1036 (m), 977 (w), 889 (w), $866(\mathrm{~m}), 826(\mathrm{~m})$, $805(\mathrm{~s}), 781$ (s), 765 (m), 721 (m), 692 (m), $674(\mathrm{~m}) \mathrm{cm}^{-1} . \mathbf{M S}$ (APCI): $\mathrm{m} / \mathrm{z}=378.1$ $\left[\mathrm{C}_{18} \mathrm{H}_{24}{ }^{79} \mathrm{BrN}_{3} \mathrm{O}+\mathrm{H}\right]^{+}$. HR-MS (EI, $\left.70 \mathrm{eV}\right)$ : calculated for $\mathrm{C}_{18} \mathrm{H}_{24}{ }^{79} \mathrm{BrN}_{3} \mathrm{O}^{+\cdot}[\mathrm{M}]^{+\cdot}: \mathrm{m} / \mathrm{z}=377.10973$, found: 377.10955 (Dev.: $-0.18 \mathrm{mu} ;-0.47 \mathrm{ppm}$ ).

\subsubsection{4-((4-((1s,3s)-Adamantan-1-yl)-1H-1,2,3-triazol-1-yl)methyl)-2- bromobenzaldehyde $(38)$}

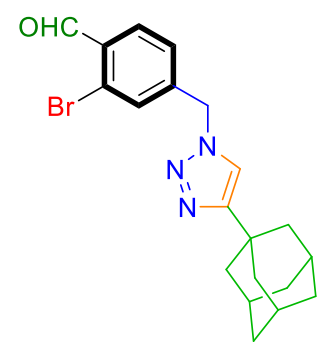

38

According to GP1 the reaction of $\mathbf{4}(24.0 \mathrm{mg}, 100 \mu \mathrm{mol}, 1.00 \mathrm{eq})$ and $\mathbf{3 1}$ (16.8 mg, $105 \mu \mathrm{mol}, 1.05 \mathrm{eq})$ provided after 24 hours reaction time and subsequent flash column chromatography $\left(\mathrm{SiO}_{2}, \mathrm{CH}: \mathrm{EE}\right.$ 3:1 v:v) $\mathbf{3 8}(38.7 \mathrm{mg}, 96.7 \mu \mathrm{mol}, 97 \%)$ as a colorless solid.

$\mathbf{R}_{f}=0.24\left(\mathrm{SiO}_{2}, \mathrm{CH}: \mathrm{EE} 3: 1 \mathrm{v}: \mathrm{v}\right)$. Mp.: $99-101{ }^{\circ} \mathrm{C} .{ }^{1} \mathbf{H}-\mathbf{N M R}\left(360 \mathbf{M H z}, \mathbf{C D C l}_{3}\right): \delta=10.31(\mathrm{~s}, 1 \mathrm{H})$, $7.88\left(\mathrm{~d},{ }^{3} J_{\mathrm{HH}}=8.0 \mathrm{~Hz}, 1 \mathrm{H}\right), 7.51\left(\mathrm{~d},{ }^{4} J_{\mathrm{HH}}=1.7 \mathrm{~Hz}, 1 \mathrm{H}\right), 7.26\left(\mathrm{dd},{ }^{3} J_{\mathrm{HH}}=8.2 \mathrm{~Hz},{ }^{4} J_{\mathrm{HH}}=1.6 \mathrm{~Hz}, 1 \mathrm{H}\right)$, $7.21(\mathrm{~s}, 1 \mathrm{H}), 5.52(\mathrm{~s}, 2 \mathrm{H}), 2.08-2.00(\mathrm{~m}, 3 \mathrm{H}), 1.95\left(\mathrm{~d},{ }^{3} \mathrm{~J}_{\mathrm{HH}}=2.9 \mathrm{~Hz}, 6 \mathrm{H}\right), 1.81-1.70(\mathrm{~m}, 6 \mathrm{H}) \mathrm{ppm}$. ${ }^{13} \mathbf{C}\{1 \mathbf{H}\}-N M R\left(91 \mathrm{MHz}, \mathbf{C D C l}_{3}\right): \delta=191.2,159.1,142.9,133.5,133.0,130.6,127.6,127.3,118.7$, 52.8, 42.6 (3x), 36.7 (3x), 32.8, 28.5 (3x) ppm. IR (ATR, neat): $\tilde{v}=2900$ (s), 2847 (m), 1689 (s), 1599 (m), 1562 (w), 1539 (w), 1450 (w), 1406 (w), 1387 (m), 1343 (w), 1303 (w), $1286(\mathrm{w}), 1266$ (m), 1205 (s), $1140(\mathrm{w}), 1101(\mathrm{w}), 1073$ (w), 1042 (s), 1002 (m), 975 (w), 925 (w), 898 (w), 845 (m), 811 (w), 788 (s), $771(\mathrm{~m}), 755(\mathrm{~s}), 724(\mathrm{~s}), 676(\mathrm{~m}) \mathrm{cm}^{-1}$. MS (APCI): $\mathrm{m} / \mathrm{z}=400.0\left[\mathrm{C}_{20} \mathrm{H}_{22}{ }^{79} \mathrm{BrN}_{3} \mathrm{O}+\mathrm{H}\right]^{+}$. HR-MS 
(ESI): calculated for $\mathrm{C}_{20} \mathrm{H}_{23}{ }^{79} \mathrm{BrN}_{3} \mathrm{O}^{+}[\mathrm{M}+\mathrm{H}]^{+}: \mathrm{m} / \mathrm{z}=400.10190$, found: 400.10166 (Dev.: $-0.24 \mathrm{mu}$; $-0.59 \mathrm{ppm})$.

\subsubsection{2-Bromo-4-((4-phenyl-1H-1,2,3-triazol-1-yl)methyl)benzaldehyde (39)}

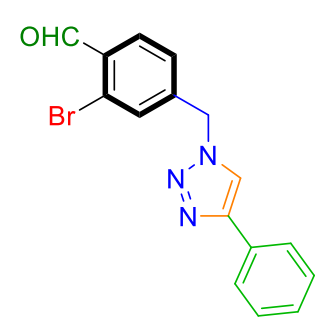

39

According to GP1 the reaction of $4(24.0 \mathrm{mg}, 100 \mu \mathrm{mol}, 1.00 \mathrm{eq})$ and phenylacetylene $(11.5 \mu \mathrm{L}$, $105 \mu \mathrm{mol}, 1.05 \mathrm{eq})$ provided after 24 hours reaction time and subsequent flash column chromatography $\left(\mathrm{SiO}_{2}, \mathrm{CH}: \mathrm{EE} 3: 1 \mathrm{v}: \mathrm{v}\right) \mathbf{3 9}(33.1 \mathrm{mg}, 96.7 \mu \mathrm{mol}, 97 \%)$ as a colorless solid.

$\mathbf{R}_{f}=0.13\left(\mathrm{SiO}_{2}, \mathrm{CH}: \mathrm{EE} 3: 1 \mathrm{v}: \mathrm{v}\right)$. Mp.: $141-143{ }^{\circ} \mathrm{C} .{ }^{1} \mathbf{H}-\mathrm{NMR}\left(\mathbf{3 6 0} \mathbf{M H z}, \mathbf{C D C l}_{3}\right): \delta=10.32\left(\mathrm{~d},{ }^{4} \mathrm{~J}_{\mathrm{HH}}\right.$ $=0.8 \mathrm{~Hz}, 1 \mathrm{H}), 7.90\left(\mathrm{~d},{ }^{3} J_{\mathrm{HH}}=8.0 \mathrm{~Hz}, 1 \mathrm{H}\right), 7.86-7.77(\mathrm{~m}, 2 \mathrm{H}), 7.77(\mathrm{~s}, 1 \mathrm{H}), 7.55\left(\mathrm{~d},{ }^{4} J_{\mathrm{HH}}=1.6 \mathrm{~Hz}\right.$, 1H), $7.44-7.39(\mathrm{~m}, 2 \mathrm{H}), 7.37-7.30(\mathrm{~m}, 2 \mathrm{H}), 5.61(\mathrm{~s}, 2 \mathrm{H}) \mathrm{ppm} .{ }^{13} \mathbf{C}\left\{{ }^{1} \mathbf{H}\right\}-\mathbf{N M R}\left(\mathbf{9 1} \mathbf{M H z}, \mathbf{C D C l}_{3}\right): \delta$ = 191.1, 148.8, 142.5, 133.7, 133.0, 130.6, 130.2, 129.0 (2x), 128.6, 127.7, 127.2, 125.8 (2x), 119.8, 53.0 ppm. IR (ATR, neat): $\tilde{v}=3084(\mathrm{w}), 2858(\mathrm{w}), 1687(\mathrm{~m}), 1584(\mathrm{~m}), 1563(\mathrm{w}), 1465(\mathrm{~m}), 1426(\mathrm{w})$, 1403 (m), 1383 (w), 1344 (w), 1224 (m), 1201 (m), 1179 (m), 1148 (w), 1084 (m), 1047 (m), 1039 (m), $978(\mathrm{~m}), 914(\mathrm{w}), 865(\mathrm{w}), 845(\mathrm{w}), 825(\mathrm{~m}), 805(\mathrm{~m}), 782(\mathrm{~m}), 763(\mathrm{~s}), 710(\mathrm{~m}), 692(\mathrm{~s}), 677(\mathrm{~m}), 654$ (m) $\mathrm{cm}^{-1}$. MS (APCI): $\mathrm{m} / \mathrm{z}=342.0\left[\mathrm{C}_{16} \mathrm{H}_{12}{ }^{79} \mathrm{BrN}_{3} \mathrm{O}+\mathrm{H}\right]^{+}$. HR-MS (EI, $70 \mathrm{eV}$ ): calculated for $\mathrm{C}_{16} \mathrm{H}_{12}{ }^{79} \mathrm{BrN}_{3} \mathrm{O}^{+\cdot}[\mathrm{M}]^{+}: \mathrm{m} / \mathrm{z}=341.01583$, found: 341.01565 (Dev.: $-0.18 \mathrm{mu} ;-0.51 \mathrm{ppm}$ ).

\subsubsection{2-Bromo-4-((4-(3,5-di-tert-butylphenyl)-1H-1,2,3-triazol-1- yl)methyl)benzaldehyde (40)}

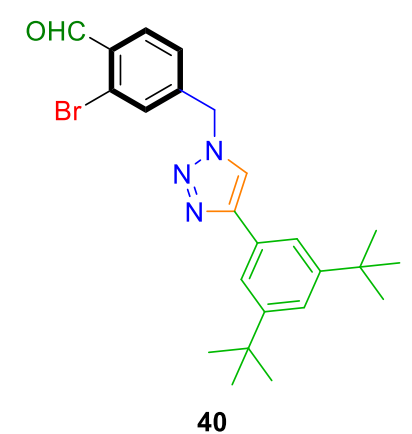

According to GP1 the reaction of $\mathbf{4}(24.0 \mathrm{mg}, 100 \mu \mathrm{mol}, 1.00 \mathrm{eq})$ and $\mathbf{3 2}(22.5 \mathrm{mg}, 105 \mu \mathrm{mol}, 1.05 \mathrm{eq})$ provided after 24 hours reaction time and subsequent flash column chromatography $\left(\mathrm{SiO}_{2}, \mathrm{CH}: \mathrm{EE}\right.$ 3:1 v:v) 40 (42.9 mg, $94.4 \mu \mathrm{mol}, 94 \%)$ as a colorless solid. 
$\mathbf{R}_{f}=0.34\left(\mathrm{SiO}_{2}, \mathrm{CH}: \mathrm{EE} 3: 1 \mathrm{v}: \mathrm{v}\right) . \mathbf{M p . :} 190-192{ }^{\circ} \mathrm{C} .{ }^{\mathbf{1}} \mathbf{H}-\mathbf{N M R}\left(\mathbf{3 6 0} \mathbf{M H z}, \mathbf{C D C l}_{3}\right): \delta=10.36(\mathrm{~s}, 1 \mathrm{H})$, $7.94\left(\mathrm{~d},{ }^{3} J_{\mathrm{HH}}=8.0 \mathrm{~Hz}, 1 \mathrm{H}\right), 7.82(\mathrm{~s}, 1 \mathrm{H}), 7.70\left(\mathrm{~d},{ }^{4} J_{\mathrm{HH}}=1.8 \mathrm{~Hz}, 2 \mathrm{H}\right), 7.58\left(\mathrm{~d},{ }^{4} J_{\mathrm{HH}}=1.7 \mathrm{~Hz}, 1 \mathrm{H}\right), 7.46$ $\left(\mathrm{t},{ }^{4} J_{\mathrm{HH}}=1.9 \mathrm{~Hz}, 1 \mathrm{H}\right), 7.35\left(\mathrm{ddd},{ }^{3} J_{\mathrm{HH}}=7.8 \mathrm{~Hz},{ }^{4} J_{\mathrm{HH}}=1.6 \mathrm{~Hz},{ }^{5} J_{\mathrm{HH}}=0.8 \mathrm{~Hz}, 1 \mathrm{H}\right), 5.66(\mathrm{~s}, 2 \mathrm{H}), 1.39(\mathrm{~s}$, 18H) ppm. ${ }^{13} \mathbf{C}\{\mathbf{1 H}\}-\mathbf{N M R}\left(\mathbf{9 1} \mathbf{~ M H z}, \mathbf{C D C l}_{3}\right): \delta=191.2,151.6(2 \mathrm{x}), 149.7,142.7,133.6,132.9,130.6$, 129.4, 127.7, 127.1, 122.9, $120.3(2 \mathrm{x}), 119.8,53.0,35.1(2 \mathrm{x}), 31.5$ (6x) ppm. IR (ATR, neat): $\tilde{v}=3104$ (w), 3067 (w), 2961 (m), 2865 (w), 1699 (s), 1599 (m), 1546 (w), 1474 (w), 1463 (w), 1444 (w), 1420 (w), 1405 (w), 1386 (m), 1363 (m), 1279 (w), 1264 (m), 1248 (m), 1224 (m), $1199(\mathrm{~m}), 1139$ (w), 1099 (w), 1054 (m), 1042 (m), 1008 (w), 882 (m), 839 (m), 781 (s), 745 (m), 714 (s), 677 (m) cm ${ }^{-1} . \mathbf{M S}$ (APCI): $\mathrm{m} / \mathrm{z}=454.1\left[\mathrm{C}_{24} \mathrm{H}_{28}{ }^{79} \mathrm{BrN}_{3} \mathrm{O}+\mathrm{H}\right]^{+}$. HR-MS (ESI): calculated for $\mathrm{C}_{24} \mathrm{H}_{29}{ }^{79} \mathrm{BrN}_{3} \mathrm{O}^{+}[\mathrm{M}+\mathrm{H}]^{+}: \mathrm{m} / \mathrm{z}$ = 454.14885, found: 454.14914 (Dev.: $0.28 \mathrm{mu} ; 0.63 \mathrm{ppm}$ ).

\subsubsection{7-Bromo-9,9-dimethyl-3-((4-octyl-1H-1,2,3-triazol-1-yl)methyl)-9H-fluorene-2- carbaldehyde (41)}

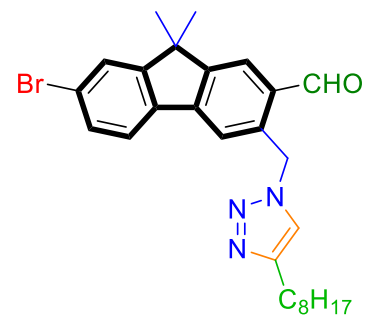

41

According to GP1 the reaction of $5(35.6 \mathrm{mg}, 100 \mu \mathrm{mol}, 1.00 \mathrm{eq})$ and 1-decyne (19.0 $\mu \mathrm{L}, 105 \mu \mathrm{mol}$, $1.05 \mathrm{eq})$ provided after 24 hours reaction time and subsequent flash column chromatography $\left(\mathrm{SiO}_{2}\right.$, CH:EE 3:1 v:v) 41 (45.2 mg, $91.4 \mu \mathrm{mol}, 91 \%)$ as a yellowish oil.

$\mathbf{R}_{f}=0.22\left(\mathrm{SiO}_{2}, \mathrm{CH}: \mathrm{EE} 3: 1 \mathrm{v}: \mathrm{v}\right) .{ }^{1} \mathbf{H}-\mathrm{NMR}\left(\mathbf{3 6 0} \mathbf{M H z}, \mathbf{C D C l}_{3}\right): \delta=10.18(\mathrm{~s}, 1 \mathrm{H}), 7.86(\mathrm{~s}, 1 \mathrm{H}), 7.59(\mathrm{~d}$, $\left.{ }^{4} J_{\mathrm{HH}}=1.7 \mathrm{~Hz}, 1 \mathrm{H}\right), 7.54\left(\mathrm{~d},{ }^{3} J_{\mathrm{HH}}=8.1 \mathrm{~Hz}, 1 \mathrm{H}\right), 7.48\left(\mathrm{dd},{ }^{3} J_{\mathrm{HH}}=8.1 \mathrm{~Hz},{ }^{4} J_{\mathrm{HH}}=1.7 \mathrm{~Hz}, 1 \mathrm{H}\right), 7.46-7.44$ $(\mathrm{m}, 2 \mathrm{H}), 6.04(\mathrm{~s}, 2 \mathrm{H}), 2.71\left(\mathrm{t},{ }^{3} J_{\mathrm{HH}}=7.6 \mathrm{~Hz}, 2 \mathrm{H}\right), 1.66\left(\mathrm{p},{ }^{3} J_{\mathrm{HH}}=7.4 \mathrm{~Hz}, 2 \mathrm{H}\right), 1.51(\mathrm{~s}, 6 \mathrm{H}), 1.39-1.18$ $(\mathrm{m}, 10 \mathrm{H}), 0.85\left(\mathrm{t},{ }^{3} \mathrm{~J}_{\mathrm{HH}}=6.8 \mathrm{~Hz}, 3 \mathrm{H}\right) \mathrm{ppm} .{ }^{13} \mathbf{C}\left\{{ }^{1} \mathbf{H}\right\}-\mathbf{N M R}\left(\mathbf{9 1} \mathbf{M H z}, \mathbf{C D C l}_{3}\right): \delta=192.7,156.6,153.2$, 148.5, 143.9, 136.4, 135.8, 131.9, 130.5, 129.3, 126.2, 123.3, 122.6, 121.5, 121.4, 50.6, 47.0, 31.6, 29.2, 29.1, 29.0, 29.0, 26.4 (2x), 25.5, 22.4, 13.9 ppm. IR (ATR, neat): $\tilde{v}=2923$ (m), 2853 (m), 1684 (s), 1614 (m), 1600 (m), 1561 (m), 1457 (m), 1405 (m), 1362 (w), 1251 (m), 1217 (w), 1179 (s), 1047 (m), 966 (m), 907 (w), 876 (w), 821 (m), 800 (m), 771 (m), 732 (s), $672(\mathrm{w}) \mathrm{cm}^{-1}$. MS (APCI): m/z = 494.1 $\left[\mathrm{C}_{27} \mathrm{H}_{32}{ }^{79} \mathrm{BrN}_{3} \mathrm{O}+\mathrm{H}\right]^{+}$. HR-MS (EI, $\left.70 \mathrm{eV}\right)$ : calculated for $\mathrm{C}_{27} \mathrm{H}_{32}{ }^{79} \mathrm{BrN}_{3} \mathrm{O}^{+\cdot}[\mathrm{M}]^{+\cdot}: \mathrm{m} / \mathrm{z}=493.17233$, found: 493.17229 (Dev.: $-0.04 \mathrm{mu} ;-0.07 \mathrm{ppm}$ ). 


\subsubsection{3-((4-((3r,5r,7r)-Adamantan-1-yl)-1H-1,2,3-triazol-1-yl)methyl)-7-bromo-9,9- dimethyl-9H-fluorene-2-carbaldehyde (42)}

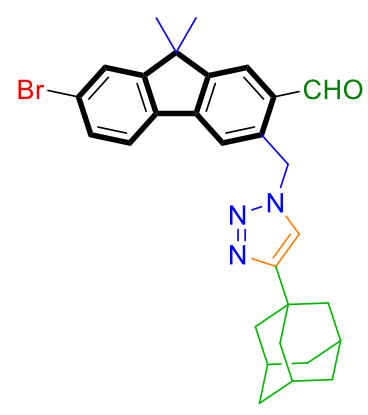

42

According to GP1 5 (107 mg, $300 \mu \mathrm{mol}, 1.00 \mathrm{eq})$ and $\mathbf{3 1}(50.5 \mathrm{mg}, 315 \mu \mathrm{mol}, 1.05 \mathrm{eq})$ were dissolved in $\mathrm{CHCl}_{3}(3.00 \mathrm{~mL}, 0.1 \mathrm{M}) . \mathrm{CuSO}_{4} \cdot 5 \mathrm{H}_{2} \mathrm{O}(7.2 \mathrm{mg}, 30 \mu \mathrm{mol}, 0.10 \mathrm{eq})$ and sodium ascorbate $(29.7 \mathrm{mg}$, $150 \mu \mathrm{mol}, 0.50 \mathrm{eq})$ were suspended separately in water $(375 \mu \mathrm{L})$ and added to the reaction vial. The heterogeneous mixture was stirred vigorously at $25{ }^{\circ} \mathrm{C}$ for three days and directly purified by flash column chromatography ( $\left.\mathrm{SiO}_{2}, \mathrm{CH}: \mathrm{EE} 3: 1 \mathrm{v}: \mathrm{v}\right) \mathbf{4 2}(153 \mathrm{mg}, 296 \mu \mathrm{mol}, 99 \%)$ as a colorless oil, which solidified upon standing at $25^{\circ} \mathrm{C}$.

$\mathbf{R}_{f}=0.33\left(\mathrm{SiO}_{2}\right.$, CH:EE 3:1 v:v). Mp.: $123-125{ }^{\circ} \mathrm{C} .{ }^{1} \mathbf{H}-\mathbf{N M R}\left(360 \mathbf{M H z}, \mathbf{C D C l}_{3}\right): \delta=10.18(\mathrm{~s}, 1 \mathrm{H})$, $7.86(\mathrm{~s}, 1 \mathrm{H}), 7.59(\mathrm{~s}, 1 \mathrm{H}), 7.53\left(\mathrm{~d},{ }^{3} J_{\mathrm{HH}}=8.0 \mathrm{~Hz}, 1 \mathrm{H}\right), 7.49\left(\mathrm{~d},{ }^{3} J_{\mathrm{HH}}=7.9 \mathrm{~Hz}, 1 \mathrm{H}\right), 7.45(\mathrm{~s}, 1 \mathrm{H}), 7.39(\mathrm{~s}$, 1H), 6.03 (s, 2H), 2.04 (s, 3H), 1.97 (s, 6H), 1.75 (s, 6H), 1.50 (s, 6H) ppm. ${ }^{13} \mathbf{C}\left\{{ }^{1} \mathbf{H}\right\}$-NMR (91 MHz, CDCl $\left._{3}\right): \delta=193.1,158.4,157.0,153.5,144.2,136.8,136.2,132.3,130.9,129.5,126.6,123.6,122.9$, 122.1, 119.4, 50.8, 47.4, 42.6 (3x), 36.8 (3x), 32.8, 28.5 (3x), 26.7 (2x) ppm. IR (ATR, neat): $\tilde{v}=2903$ (m), 2847 (m), 1677 (s), 1615 (m), 1563 (m), 1448 (m), 1427 (m), 1408 (w), 1361 (w), 1344 (w), 1312 (w), $1253(\mathrm{~m}), 1213(\mathrm{w}), 1182(\mathrm{~m}), 1167$ (m), $1128(\mathrm{w}), 1101(\mathrm{w}), 1081(\mathrm{w}), 1063(\mathrm{~m}), 1049(\mathrm{~m}), 1001$ (w), 967 (m), $905(\mathrm{~m}), 880(\mathrm{w}), 827(\mathrm{~s}), 806(\mathrm{~m}), 789(\mathrm{~m}), 767$ (m), 726 (s), $672(\mathrm{~m}) \mathrm{cm}^{-1}$. MS (APCI): $\mathrm{m} / \mathrm{z}=516.1\left[\mathrm{C}_{29} \mathrm{H}_{30}{ }^{79} \mathrm{BrN}_{3} \mathrm{O}+\mathrm{H}\right]^{+}$. HR-MS (APCI): calculated for $\mathrm{C}_{29} \mathrm{H}_{31}{ }^{79} \mathrm{BrN}_{3} \mathrm{O}^{+}[\mathrm{M}+\mathrm{H}]^{+}: \mathrm{m} / \mathrm{z}=$ 516.16450, found: 516.16445 (Dev.: $-0.05 \mathrm{mu} ;-0.10 \mathrm{ppm}$ ).

\subsubsection{7-Bromo-9,9-dimethyl-3-((4-phenyl-1H-1,2,3-triazol-1-yl)methyl)-9H-fluorene-2- carbaldehyde (43)}

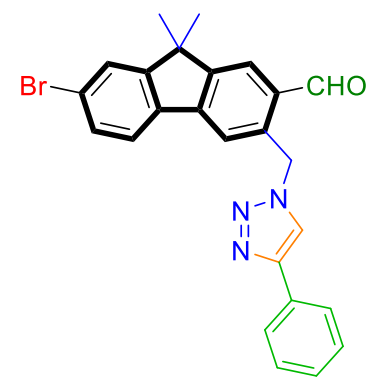


According to GP1 the reaction of 5 (35.6 mg, $100 \mu \mathrm{mol}, 1.00 \mathrm{eq})$ and phenylacetylene $(11.5 \mu \mathrm{L}$, $105 \mu \mathrm{mol}, 1.05 \mathrm{eq})$ provided after 48 hours reaction time and subsequent flash column chromatography $\left(\mathrm{SiO}_{2}, \mathrm{CH}: \mathrm{EE} 3: 1 \mathrm{v}: \mathrm{v}\right) \mathbf{4 3}$ (42.9 mg, $\left.93.6 \mu \mathrm{mol}, 94 \%\right)$ as a colorless foam.

$\mathbf{R}_{f}=0.24\left(\mathrm{SiO}_{2}, \mathrm{CH}: \mathrm{EE} \mathrm{3:1} \mathrm{v:v).} \mathrm{Mp.:} 76-86^{\circ} \mathrm{C}\right.$ (very long melting). ${ }^{1} \mathbf{H}-\mathbf{N M R}\left(\mathbf{3 6 0} \mathbf{M H z}, \mathbf{C D C l}_{3}\right)$ : $\delta=10.19(\mathrm{~s}, 1 \mathrm{H}), 7.98(\mathrm{~s}, 1 \mathrm{H}), 7.87(\mathrm{~s}, 1 \mathrm{H}), 7.88-7.79(\mathrm{~m}, 2 \mathrm{H}), 7.61-7.59(\mathrm{~m}, 2 \mathrm{H}), 7.57\left(\mathrm{~d},{ }^{3} J_{\mathrm{HH}}=\right.$ $8.2 \mathrm{~Hz}, 1 \mathrm{H}), 7.48\left(\mathrm{dd},{ }^{3} J_{\mathrm{HH}}=8.2 \mathrm{~Hz},{ }^{4} J_{\mathrm{HH}}=1.7 \mathrm{~Hz}, 1 \mathrm{H}\right), 7.43-7.37(\mathrm{~m}, 2 \mathrm{H}), 7.34-7.28(\mathrm{~m}, 1 \mathrm{H}), 6.13$

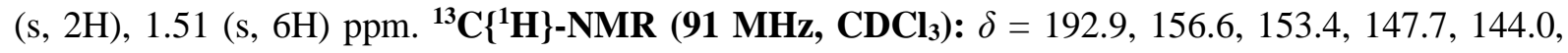
135.8, 135.7, 132.0, 130.6, 130.3, 129.6, $128.6(2 \mathrm{x}), 127.9,126.2,125.5(2 \mathrm{x}), 123.4,122.7,122.0,120.4$, 50.8, 47.1, 26.4 (2x) ppm. IR (ATR, neat): $\tilde{v}=2961$ (w), 2924 (w), 2858 (w), 1683 (s), 1614 (m), 1600 (w), 1561 (m), 1463 (w), 1406 (w), 1251 (m), 1179 (m), 1076 (m), 1045 (m), 965 (m), 907 (w), 822 (m), $810(\mathrm{~m}), 763$ (s), $732(\mathrm{~s}), 694(\mathrm{~s}), 672(\mathrm{~m}) \mathrm{cm}^{-1}$. MS (APCI): $\mathrm{m} / \mathrm{z}=458.0\left[\mathrm{C}_{25} \mathrm{H}_{20}{ }^{79} \mathrm{BrN}_{3} \mathrm{O}+\mathrm{H}\right]^{+}$. HR-MS (EI, $70 \mathrm{eV}$ ): calculated for $\mathrm{C}_{25} \mathrm{H}_{20}{ }^{79} \mathrm{BrN}_{3} \mathrm{O}^{+\cdot}[\mathrm{M}]^{+*}: \mathrm{m} / \mathrm{z}=457.07843$, found: 457.07831 (Dev.: $-0.12 \mathrm{mu} ;-0.25 \mathrm{ppm})$.

\subsubsection{7-Bromo-3-((4-(3,5-di-tert-butylphenyl)-1H-1,2,3-triazol-1-yl)methyl)-9,9- dimethyl-9H-fluorene-2-carbaldehyde (44)}

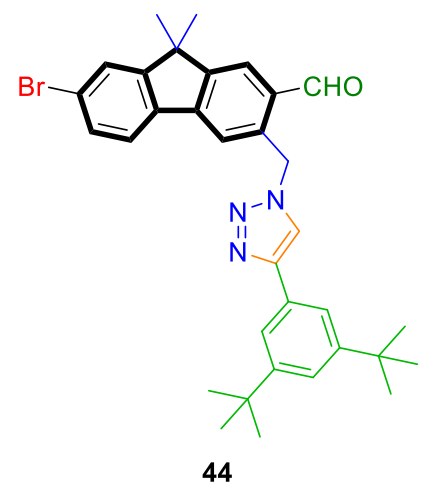

According to GP1 the reaction of $\mathbf{5}(35.6 \mathrm{mg}, 100 \mu \mathrm{mol}, 1.00 \mathrm{eq})$ and $\mathbf{3 2}(22.5 \mathrm{mg}, 105 \mu \mathrm{mol}, 1.05 \mathrm{eq})$ provided after six days reaction time and subsequent flash column chromatography $\left(\mathrm{SiO}_{2}, \mathrm{CH}: \mathrm{EE}\right.$ 3:1 v:v) 44 (55.9 mg, $98.0 \mu \mathrm{mol}, 98 \%)$ as a colorless solid.

$\mathbf{R}_{f}=0.47\left(\mathrm{SiO}_{2}, \mathrm{CH}: \mathrm{EE} 3: 1 \mathrm{v}: \mathrm{v}\right) . \mathbf{M p} .: 210-212{ }^{\circ} \mathrm{C} .{ }^{1} \mathbf{H}-\mathbf{N M R}\left(360 \mathrm{MHz}, \mathbf{C D C l}_{3}\right): \delta=10.21(\mathrm{~s}, 1 \mathrm{H})$, $7.97(\mathrm{~s}, 1 \mathrm{H}), 7.88(\mathrm{~s}, 1 \mathrm{H}), 7.70(\mathrm{~d}, \mathrm{~J}=1.8 \mathrm{~Hz}, 2 \mathrm{H}), 7.60(\mathrm{~d}, \mathrm{~J}=1.7 \mathrm{~Hz}, 1 \mathrm{H}), 7.57(\mathrm{~d}, \mathrm{~J}=8.2 \mathrm{~Hz}, 1 \mathrm{H})$, 7.54 (s, 1H), 7.48 (dd, J = 8.2, 1.8 Hz, 1H), 7.42 (t, J = 1.8 Hz, 1H), 6.16 (s, 2H), 1.52 (s, 6H), 1.36 (s, $18 \mathrm{H}) \mathrm{ppm} .{ }^{13} \mathbf{C}\left\{{ }^{1} \mathbf{H}\right\}$-NMR (91 MHz, $\left.\mathbf{C D C l}\right): \delta=193.3,157.0,153.6,151.4(2 \mathrm{x}), 149.0,144.3,136.6$, 136.1, 132.2, 130.9, 129.8 (2x), 126.6, 123.7, 123.1, 122.6, 121.8, 120.6, 120.2 (2x), 51.1, 47.4, 35.1 (2x), 31.6 (6x), 26.7 (2x) ppm. IR (ATR, neat): $\tilde{v}=2960$ (m), 2903 (w), $2866(\mathrm{w}), 1686(\mathrm{~s}), 1613$ (m), 1600 (m), 1563 (m), 1462 (m), 1420 (m), 1393 (m), 1362 (s), 1281 (m), 1250 (s), 1224 (m), 1179 (s), 1091 (m), 1047 (m), 967 (m), 900 (m), 876 (s), 847 (m), 821 (s), 810 (s), 730 (s), 708 (s), 673 (m), 661 
(m) $\mathrm{cm}^{-1}$. MS (APCI): $\mathrm{m} / \mathrm{z}=570.1\left[\mathrm{C}_{33} \mathrm{H}_{36}{ }^{79} \mathrm{BrN}_{3} \mathrm{O}+\mathrm{H}\right]^{+}$. HR-MS (APCI): calculated for $\mathrm{C}_{33} \mathrm{H}_{37}{ }^{79} \mathrm{BrN}_{3} \mathrm{O}^{+}[\mathrm{M}+\mathrm{H}]^{+}: \mathrm{m} / \mathrm{z}=570.21145$, found: 570.21154 (Dev.: $0.09 \mathrm{mu} ; 0.15 \mathrm{ppm}$ ).

\subsection{Methylation Reaction of Adamantyl substituted triazole 42}

\subsubsection{4-((3r,5r,7r)-Adamantan-1-yl)-1-((7-bromo-2-formyl-9,9-dimethyl-9H-fluoren-3- yl)methyl)-3-methyl-1H-1,2,3-triazol-3-ium tetrafluoroborate (45)}

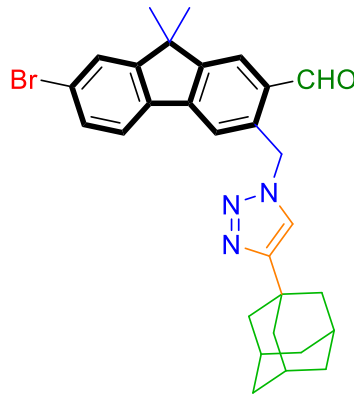

42

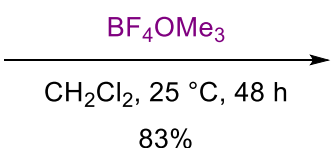

$83 \%$

A modified literature procedure was used.[35] A heat gun-dried and nitrogen-flushed Schlenk flask, equipped with a magnetic stirring bar and a rubber septum, was charged with $\mathbf{4 2}$ (25.8 mg, $50.0 \mu \mathrm{mol}$, $1.00 \mathrm{eq})$ and dissolved in anhydrous $\mathrm{CH}_{2} \mathrm{Cl}_{2}(3.33 \mathrm{~mL}, 15 \mathrm{mM})$. Trimethyloxonium tetrafluoroborate (7.40 mg, $50.0 \mu \mathrm{mol}, 1.00 \mathrm{eq}$ ) was added in one portion at $25^{\circ} \mathrm{C}$ whereupon the mixture turned slightly yellow. After stirring for two days under these conditions, methanol $(1.0 \mathrm{~mL})$ was added and the mixture was stirred for 30 minutes under air. All volatile components were removed under reduced pressure and the oily yellow residue was purified by flash column chromatography $\left(\mathrm{CH}_{2} \mathrm{Cl}_{2}: \mathrm{MeOH} 15: 1 \mathrm{v}\right.$ :v) to provide 45 (25.7 mg, $41.6 \mu \mathrm{mol}, 83 \%)$ as a colorless solid. If desired the product can be precipitated by dissolving in $\mathrm{CH}_{2} \mathrm{Cl}_{2}$ and addition of diethyl ether until a white precipitate is formed.

$\mathbf{R}_{f}=0.33\left(\mathrm{SiO}_{2}\right.$, DCM:MeOH 15:1 v:v). Mp.: $249-251^{\circ} \mathrm{C}$ decomp. ${ }^{\mathbf{1}} \mathbf{H}-\mathbf{N M R}\left(\mathbf{3 6 0} \mathbf{~ M H z}, \mathbf{C D C l}_{\mathbf{3}}\right)$ : $\delta=10.10(\mathrm{~s}, 1 \mathrm{H}), 8.22(\mathrm{~s}, 1 \mathrm{H}), 8.20(\mathrm{~s}, 1 \mathrm{H}), 7.92(\mathrm{~s}, 1 \mathrm{H}), 7.86\left(\mathrm{~d},{ }^{3} J_{\mathrm{HH}}=8.1 \mathrm{~Hz}, 1 \mathrm{H}\right), 7.59\left(\mathrm{~d},{ }^{4} J_{\mathrm{HH}}=\right.$ $1.7 \mathrm{~Hz}, 1 \mathrm{H}), 7.52\left(\mathrm{dd},{ }^{3} J_{\mathrm{HH}}=8.2 \mathrm{~Hz},{ }^{4} J_{\mathrm{HH}}=1.8 \mathrm{~Hz}, 1 \mathrm{H}\right), 6.17(\mathrm{~s}, 2 \mathrm{H}), 4.31(\mathrm{~s}, 3 \mathrm{H}), 2.11(\mathrm{~s}, 3 \mathrm{H}), 2.07-$ $2.02(\mathrm{~m}, 6 \mathrm{H}), 1.78-1.72(\mathrm{~m}, 6 \mathrm{H}), 1.53(\mathrm{~s}, 6 \mathrm{H}) \mathrm{ppm} .{ }^{13} \mathbf{C}\left\{{ }^{1} \mathbf{H}\right\}-\mathbf{N M R}\left(\mathbf{9 1} \mathbf{M H z}, \mathbf{C D C l}_{3}\right): \delta=193.1$, 158.7, 156.8, 155.4, 151.0, 145.0, 136.0, 133.0, 131.2, 130.7, 130.0, 128.2, 126.4, 126.3, 124.0, 54.7, 47.6, 41.0, 39.5 (3x), 35.8 (3x), 33.8, 27.7 (3x), 26.6 (2x) ppm. IR (ATR, neat): $\tilde{v}=2909$ (m), 2850 (w), 2734 (w), 1703 (s), 1615 (w), 1600 (w), 1562 (m), 1456 (w), 1406 (w), 1380 (w), 1356 (w), 1325 (w), $1311(\mathrm{w}), 1284(\mathrm{w}), 1251(\mathrm{w}), 1251(\mathrm{w}), 1231(\mathrm{w}), 1180(\mathrm{~m}), 1166(\mathrm{w}), 1137(\mathrm{w}), 1106(\mathrm{~m}), 1057$ (s), 1026 (s), 963 (m), $884(\mathrm{~m}), 867$ (w), 827 (s), $811(\mathrm{~m}), 785$ (m), $773(\mathrm{~m}), 736(\mathrm{~m}), 700(\mathrm{w}), 674(\mathrm{w})$, 655 (w) $\mathrm{cm}^{-1}$. MS (ESI): $\mathrm{m} / \mathrm{z}=530.2\left[\mathrm{C}_{30} \mathrm{H}_{33}{ }^{79} \mathrm{BrN}_{3} \mathrm{O}\right]^{+}$. HR-MS (ESI): calculated for $\mathrm{C}_{30} \mathrm{H}_{33}{ }^{79} \mathrm{BrN}_{3} \mathrm{O}^{+}$ $[\mathrm{M}]^{+}: \mathrm{m} / \mathrm{z}=530.18015$, found: 530.17960 (Dev.: $-0.55 \mathrm{mu} ;-1.04 \mathrm{ppm}$ ). 


\section{Optical Properties}

\subsection{2-(Azidomethyl)-4-bromobenzaldehyde (3) \& Derivatives}

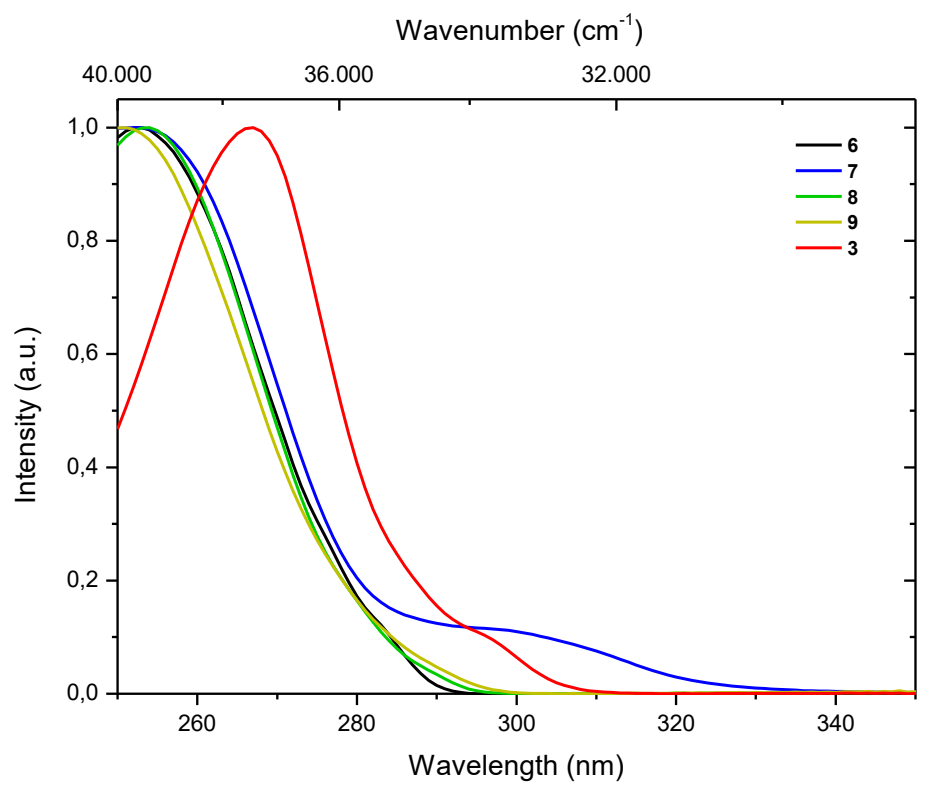

Figure S1: Normalized absorption spectra of 6 (black line), 7 (blue line), 8 (green line), 9 (yellow line) and 3 (red line) in chloroform solution $\left(c=10^{-5} \mathrm{~mol} \mathrm{~L}^{-1}\right)$.

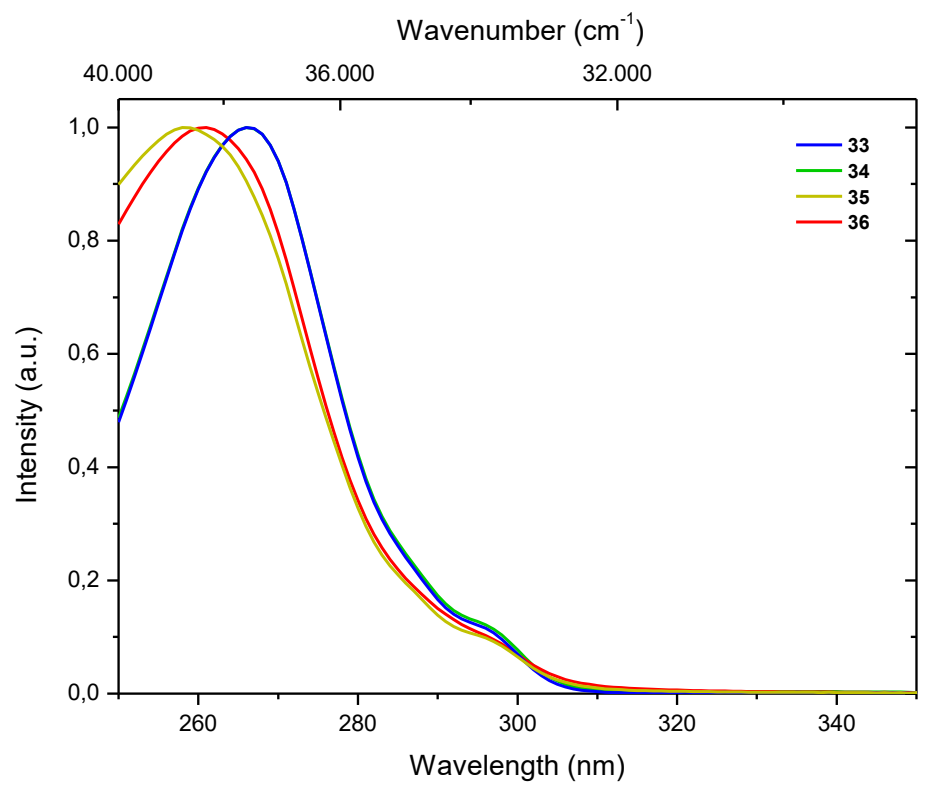

Figure S2: Normalized absorption spectra of $\mathbf{3 3}$ (blue line), $\mathbf{3 4}$ (green line), $\mathbf{3 5}$ (yellow line) and $\mathbf{3 6}$ (red line) in chloroform solution $\left(c=10^{-5} \mathrm{~mol} \mathrm{~L}^{-1}\right)$. 


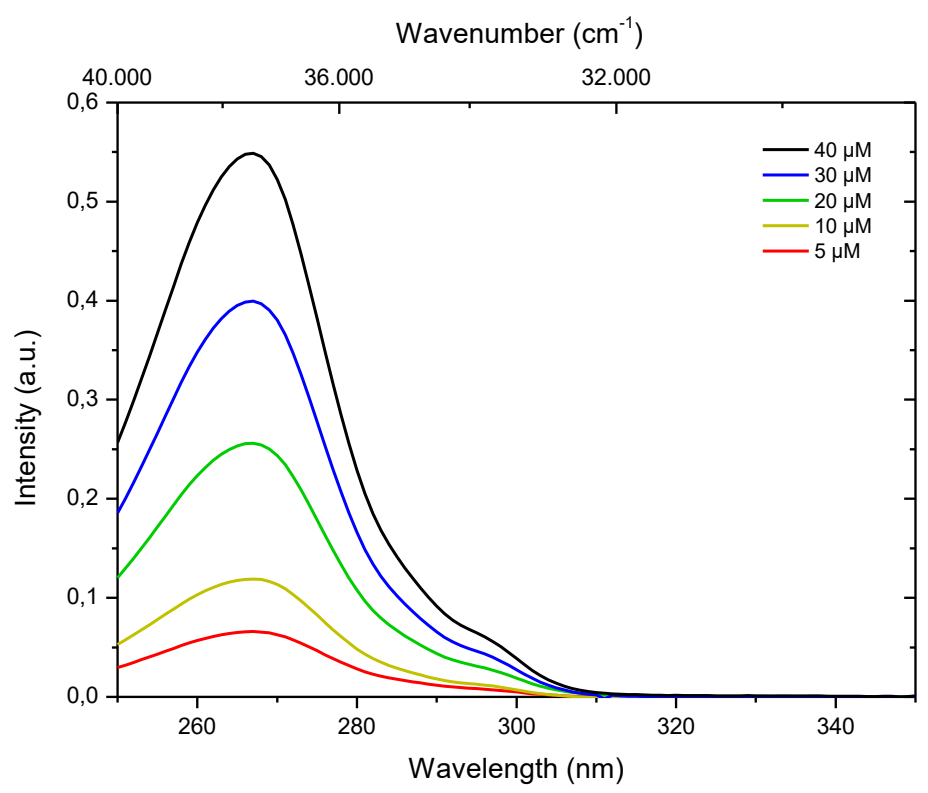

Figure S3: Absorption spectra of $\mathbf{3}$ at various concentration in chloroform solution.

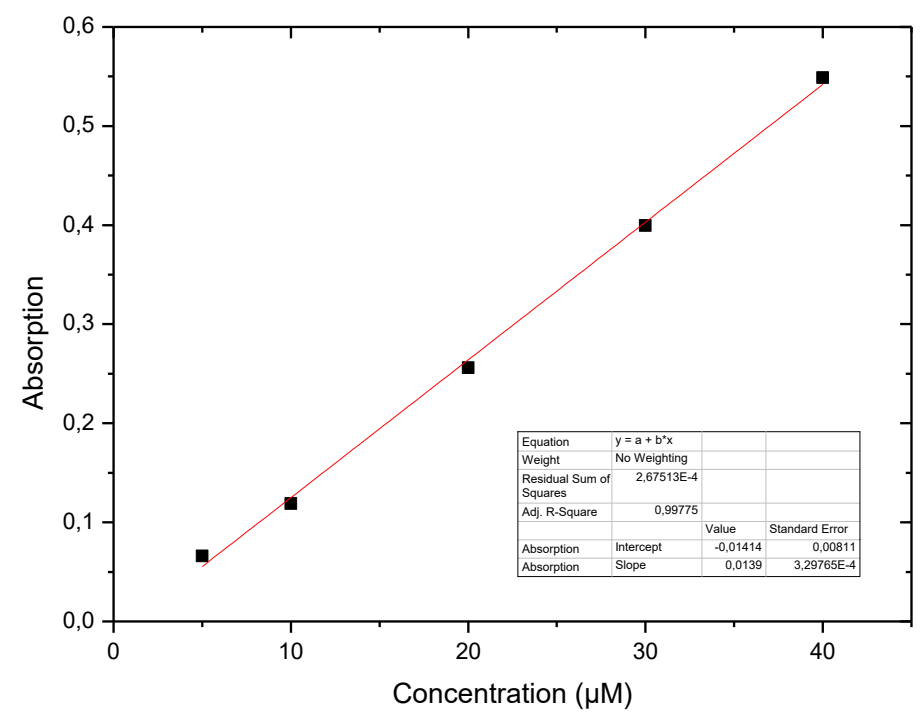

Figure S4: Linear regression for extinction coefficient determination (with respect to Figure S3): $\varepsilon_{267}=13.900 \mathrm{~L}$ $\mathrm{mol}^{-1} \mathrm{~cm}^{-1}$. 


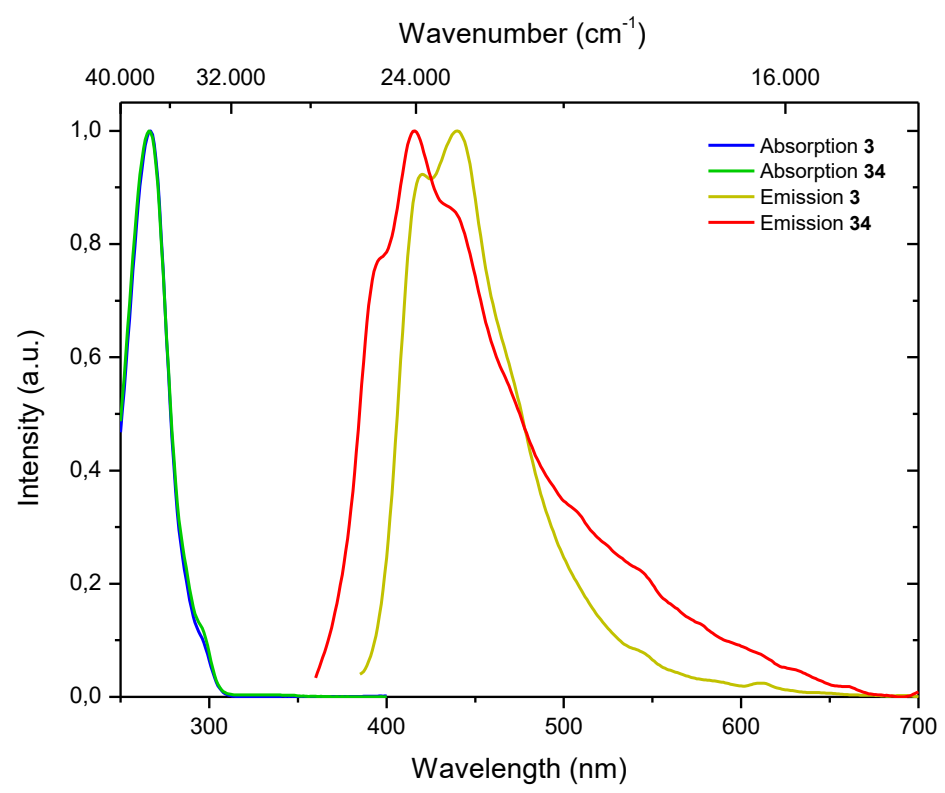

Figure S5: Normalized absorption spectra of $\mathbf{3}$ (blue line) and $\mathbf{3 4}$ (green line) in chloroform solution ( $c=10^{-5} \mathrm{~mol}$ $\mathrm{L}^{-1}$ ) after bubbling with argon for 30 minutes and normalized emission spectra of $\mathbf{3}$ (yellow line), $\mathbf{3 4}$ (red line) in the solid-state.

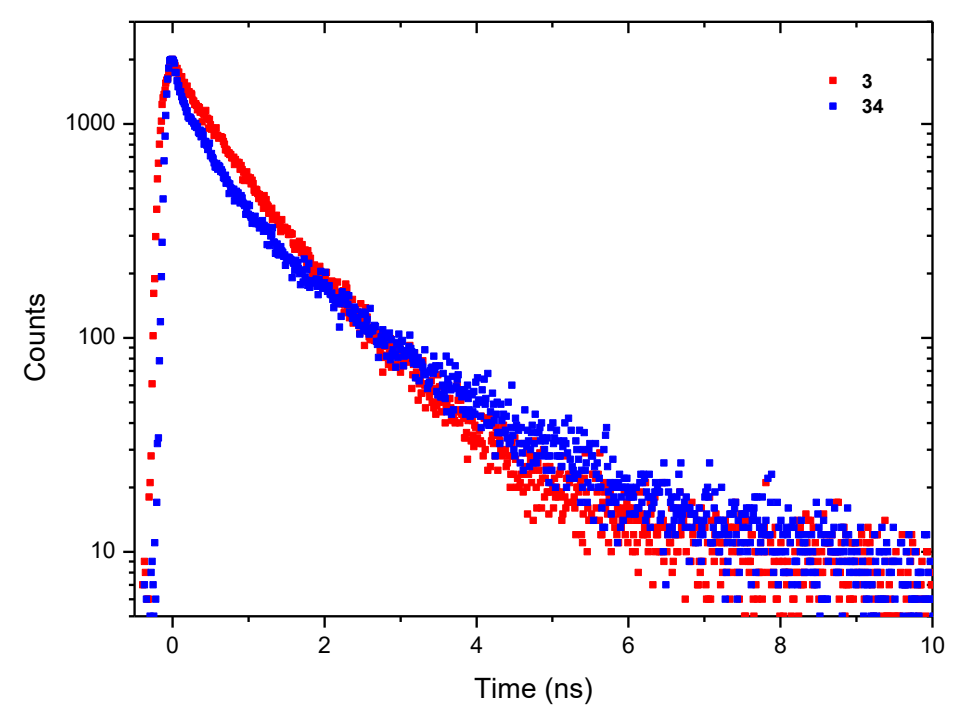

Figure S6: Fluorescence decay profile of $\mathbf{3}$ and $\mathbf{3 4}$ in the solid-state with excitation at $376 \mathrm{~nm}$.

3: $\tau_{1}=0.796 \pm 0.006(84.4 \%) \mathrm{ns} ; \tau_{2}=3.228 \pm 0.115(15.6 \%) \mathrm{ns} ; \chi^{2}=1.081$.

34: $\tau_{1}=0.516 \pm 0.006(50.2 \%) \mathrm{ns} ; \tau_{2}=2.212 \pm 0.022(49.8 \%) \mathrm{ns} ; \chi^{2}=1.069$. 


\subsection{4-(Azidomethyl)-2-bromobenzaldehyde (4) \& Derivatives}

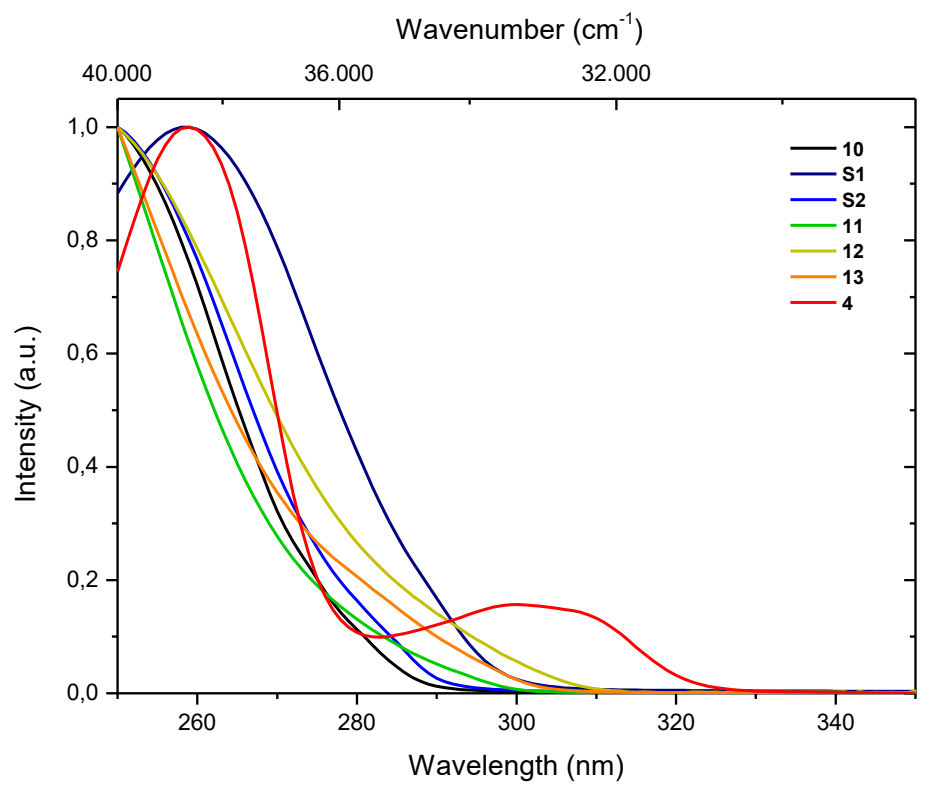

Figure S7: Normalized absorption spectra of $\mathbf{1 0}$ (black line), S1 (navy line), S2 (blue line), 11 (green line), 12 (yellow line), $\mathbf{1 3}$ (orange line) and $\mathbf{4}$ (red line) in chloroform solution $\left(c=10^{-5} \mathrm{~mol} \mathrm{~L}^{-1}\right)$.

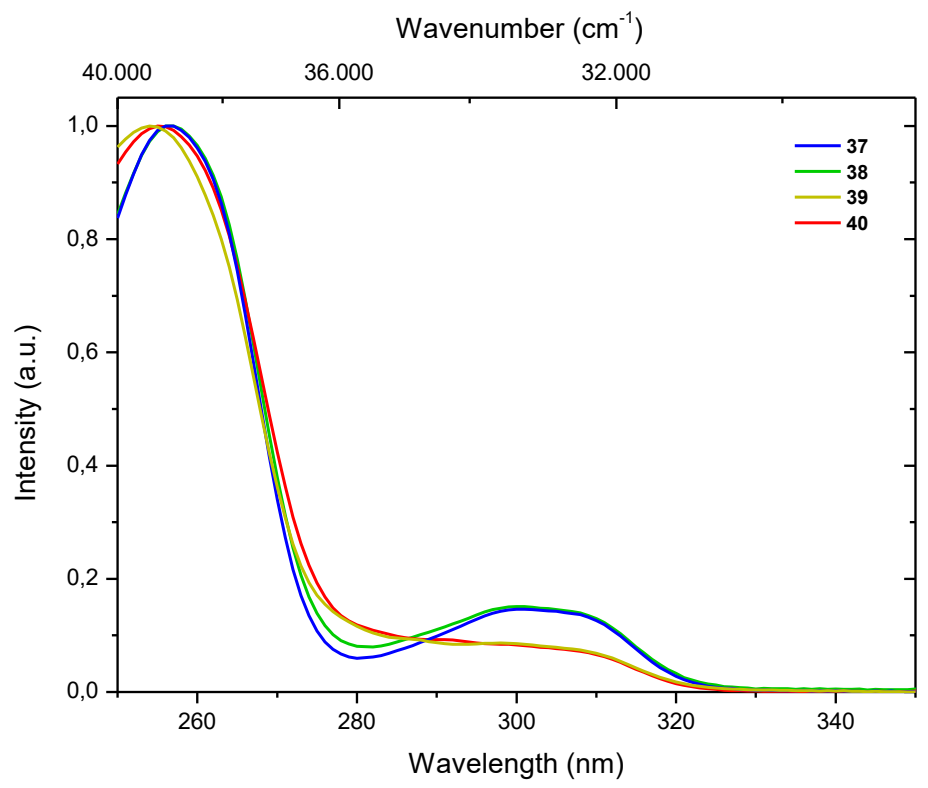

Figure S8: Normalized absorption spectra of $\mathbf{3 7}$ (blue line), $\mathbf{3 8}$ (green line), $\mathbf{3 9}$ (yellow line), $\mathbf{4 0}$ (red line) in chloroform solution $\left(c=10^{-5} \mathrm{~mol} \mathrm{~L}^{-1}\right)$. 


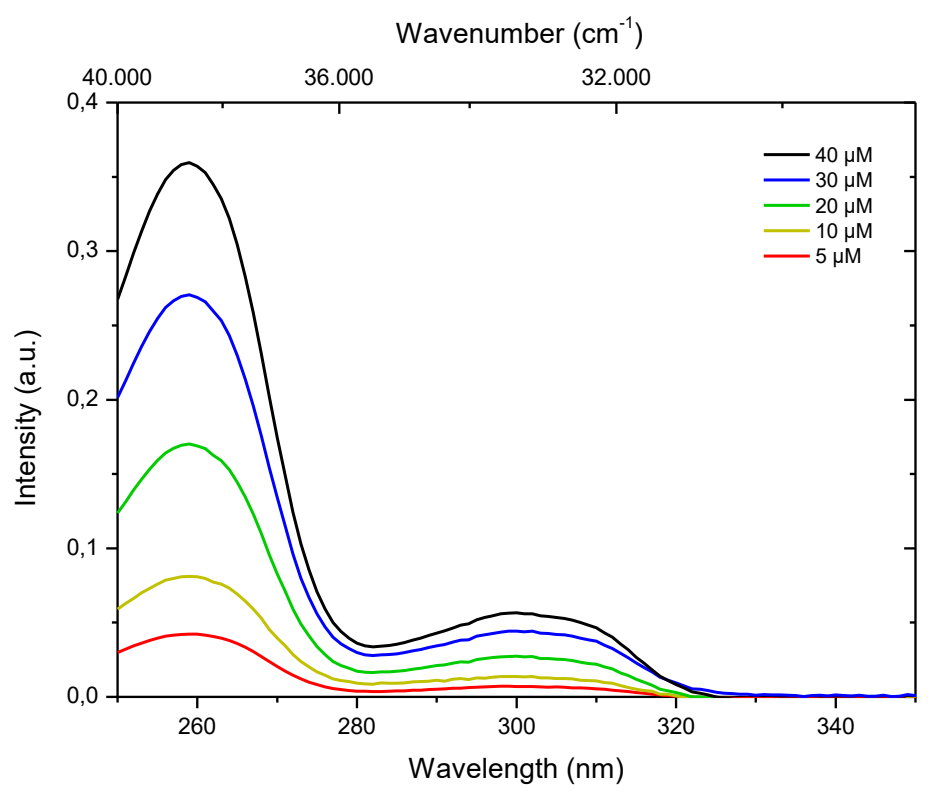

Figure S9: Absorption spectra of $\mathbf{4}$ at various concentration in chloroform solution.

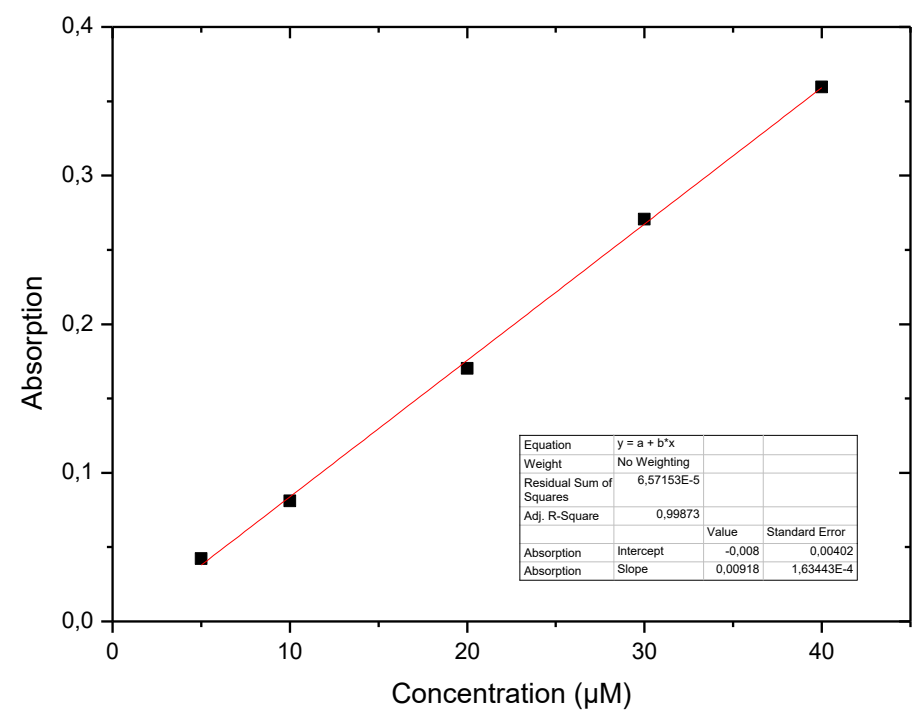

Figure S10: Linear regression for extinction coefficient determination (with respect to Figure S9): $\varepsilon_{259}=9.180 \mathrm{~L}$ $\mathrm{mol}^{-1} \mathrm{~cm}^{-1}$. 


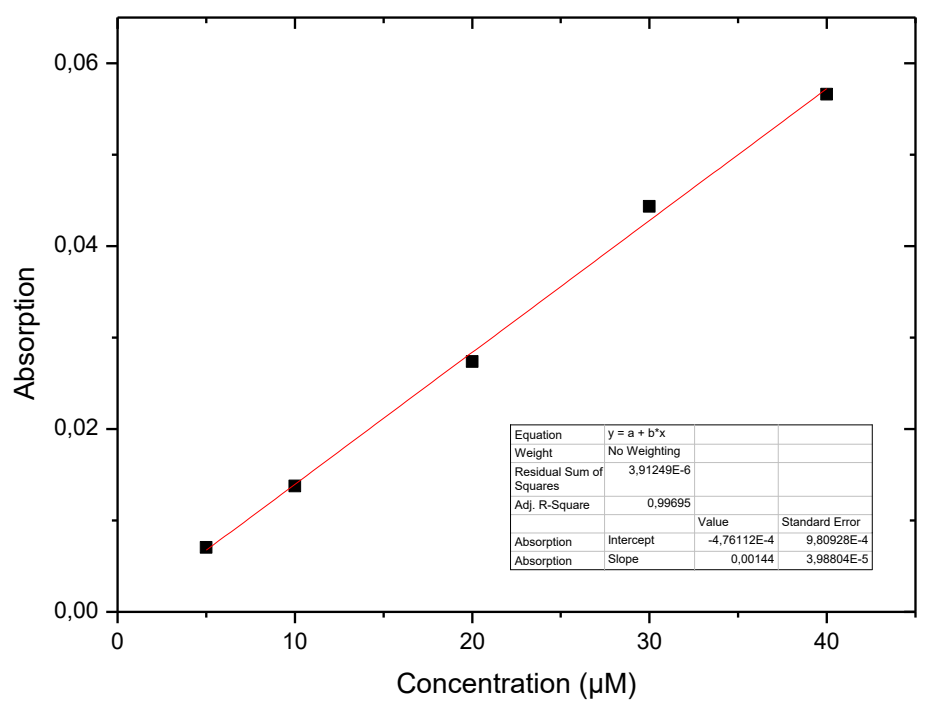

Figure S11: Linear regression for extinction coefficient determination (with respect to Figure S9): $\varepsilon_{300}=1.440 \mathrm{~L}$ $\mathrm{mol}^{-1} \mathrm{~cm}^{-1}$.

\subsection{3-(Azidomethyl)-7-bromo-9,9-dimethyl-9H-fluorene-2-carbaldehyde (5) \& Derivatives}

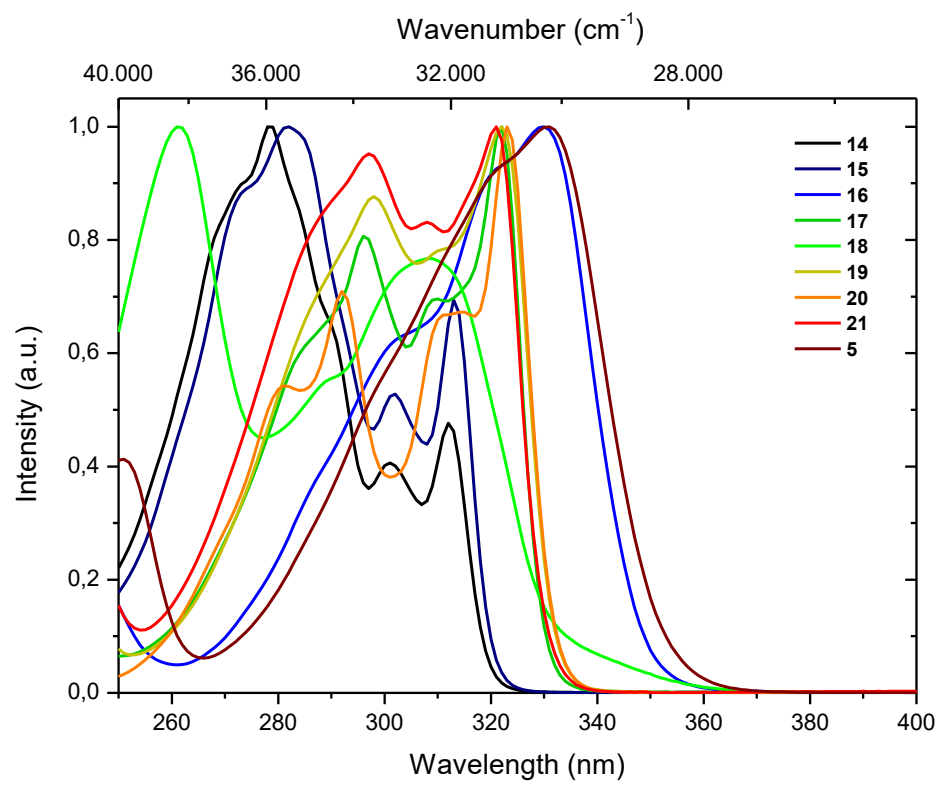

Figure S12: Normalized absorption spectra of 14 (black line), 15 (navy line), 16 (blue line), 17 (green line), 18 (light green line), 19 (yellow line), 20 (orange line), 21 (red line) and $\mathbf{6}$ (wine line) in chloroform solution $\left(c=10^{-5} \mathrm{~mol} \mathrm{~L}^{-1}\right)$. 


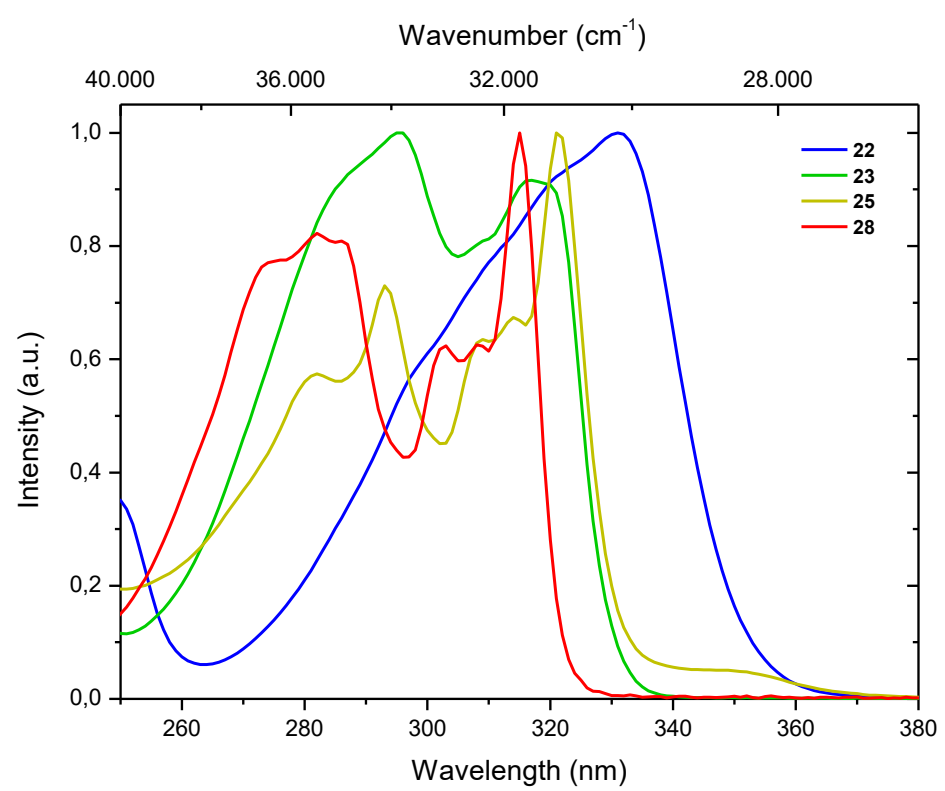

Figure S13: Normalized absorption spectra of $\mathbf{2 2}$ (blue line), $\mathbf{2 3}$ (green line), $\mathbf{2 5}$ (yellow line), $\mathbf{2 8}$ (red line) in chloroform solution $\left(c=10^{-5} \mathrm{~mol} \mathrm{~L}^{-1}\right)$.

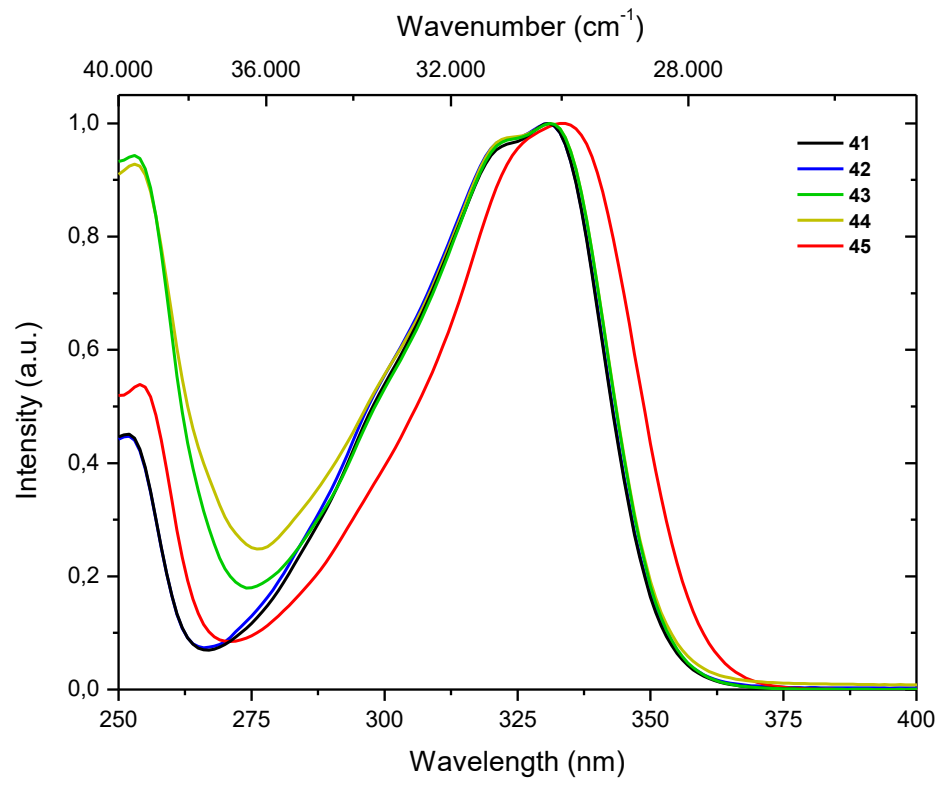

Figure S14: Normalized absorption spectra of 41 (black line), 42 (blue line), 43 (green line), 44 (yellow line), 45 (red line) in chloroform solution $\left(c=10^{-5} \mathrm{~mol} \mathrm{~L}^{-1}\right)$. 


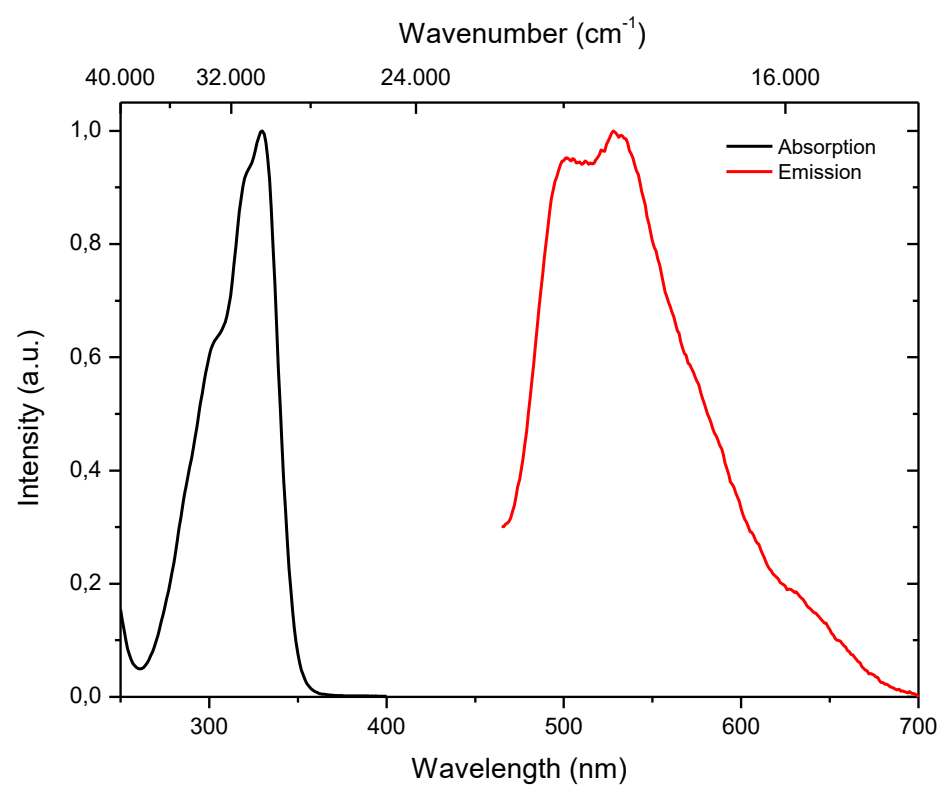

Figure S15: Normalized absorption (black line) and emission (red line) spectra of $\mathbf{1 6}$ in chloroform solution $\left(c=10^{-5} \mathrm{~mol} \mathrm{~L}^{-1}\right)$ after bubbling with argon for 30 minutes. Emission was monitored by excitation at the absorption maximum wavelength of $330 \mathrm{~nm}$. Emission maximum at $528 \mathrm{~nm}$.

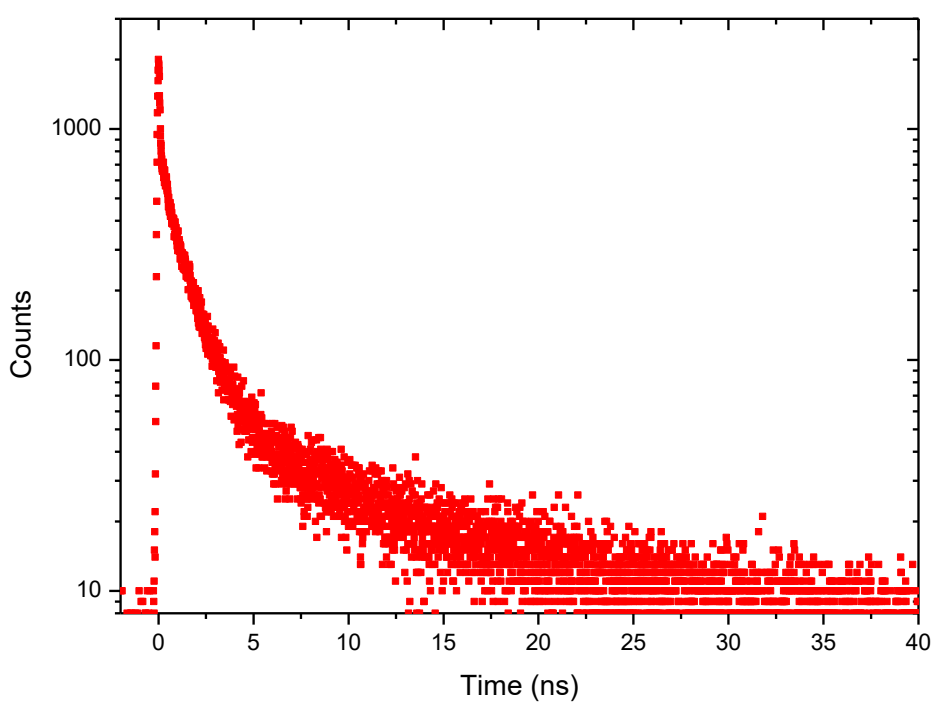

Figure S16: Fluorescence decay profile of 16 in chloroform solution $\left(c=10^{-5} \mathrm{~mol} \mathrm{~L}^{-1}\right)$ after bubbling with argon for 30 minutes with excitation at $376 \mathrm{~nm}: \tau_{1}=1.186 \pm 0.010(54.7 \%) \mathrm{ns} ; \tau_{2}=11.50 \pm 0.26(45.3 \%) \mathrm{ns} ; \chi^{2}=1.247$. Emission was detected at $530 \mathrm{~nm}$. 


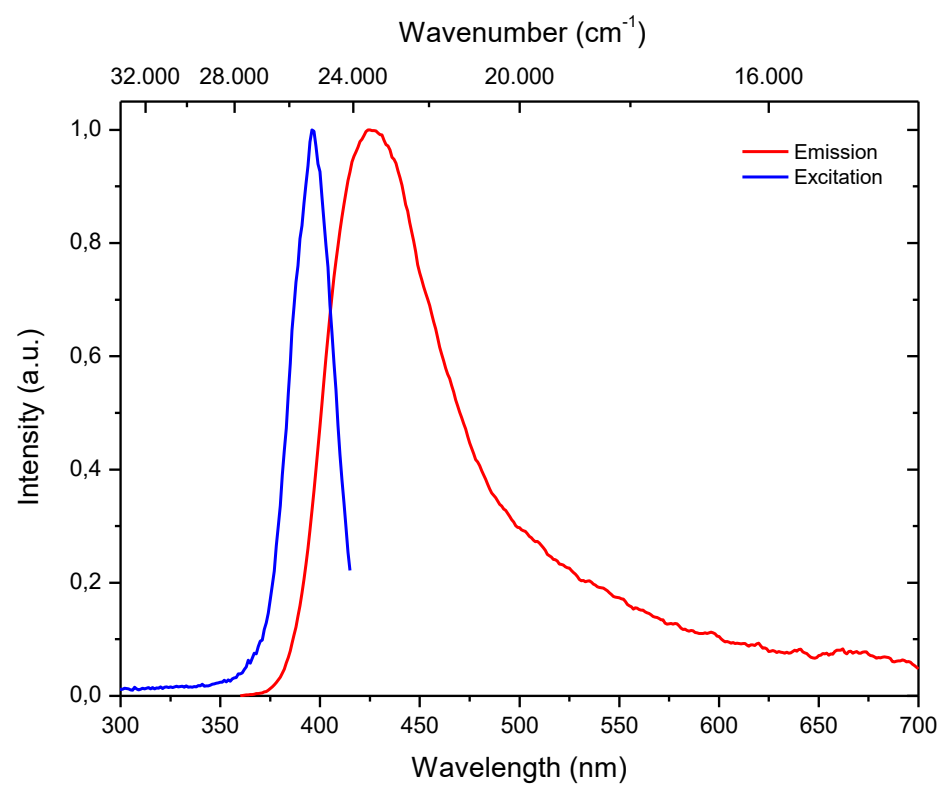

Figure S17: Normalized excitation (blue line) and emission (red line) spectra of $\mathbf{1 6}$ in the solid-state. Emission was monitored by excitation at the absorption wavelength of $396 \mathrm{~nm}$ and excitation was monitored at fluorescence maximum wavelength of $424 \mathrm{~nm}$.

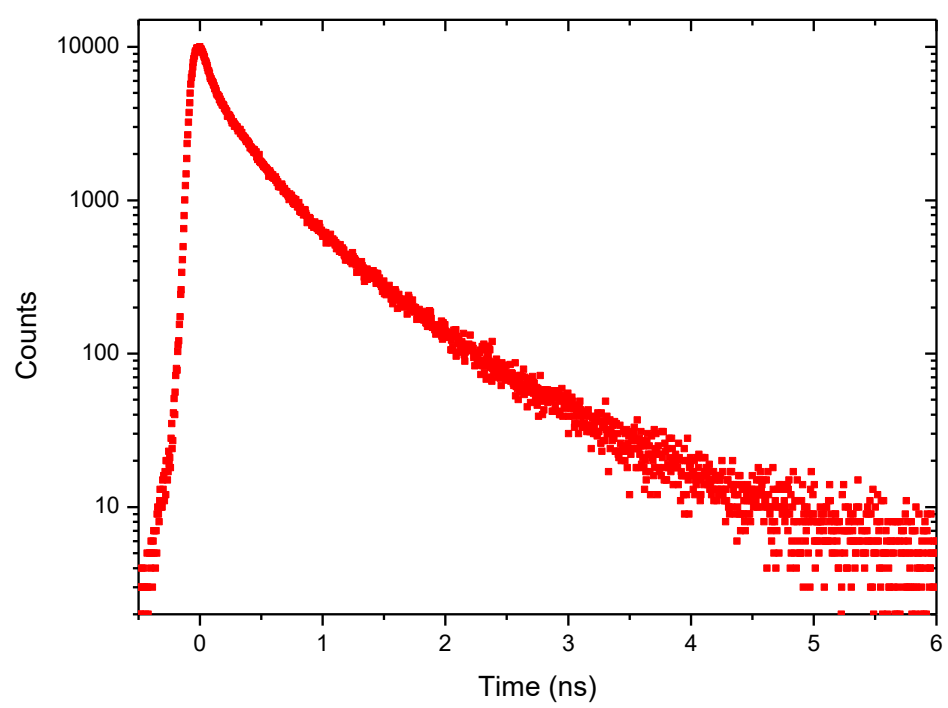

Figure S18: Fluorescence decay profile of $\mathbf{1 6}$ in the solid-state with excitation at $376 \mathrm{~nm}: \tau_{1}=0.286 \pm 0.002$ $(54.4 \%) \mathrm{ns} ; \tau_{2}=0.872 \pm 0.006(45.6 \%) \mathrm{ns} ; \chi^{2}=1.283$. 


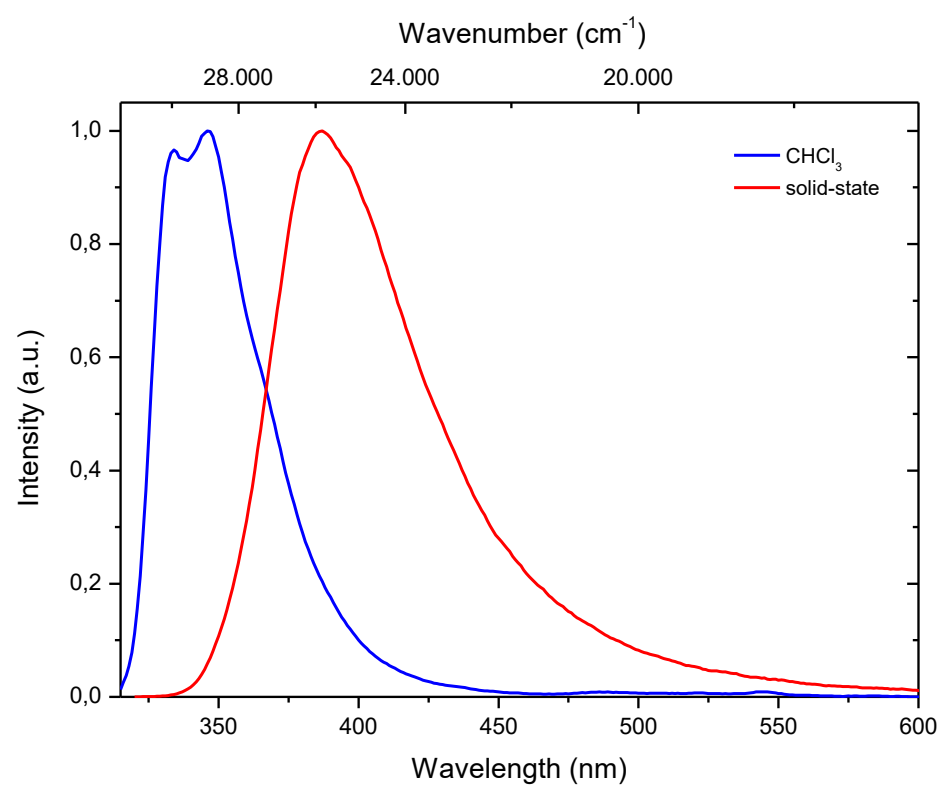

Figure S19: Normalized emission spectra of 19 in chloroform solution (blue line, $c=10^{-5} \mathrm{~mol} \mathrm{~L}^{-1}$ ) after bubbling with argon for 30 minutes and the solid-state (red line). Quantum yield in the solid-state was determined to $6.0 \%$.

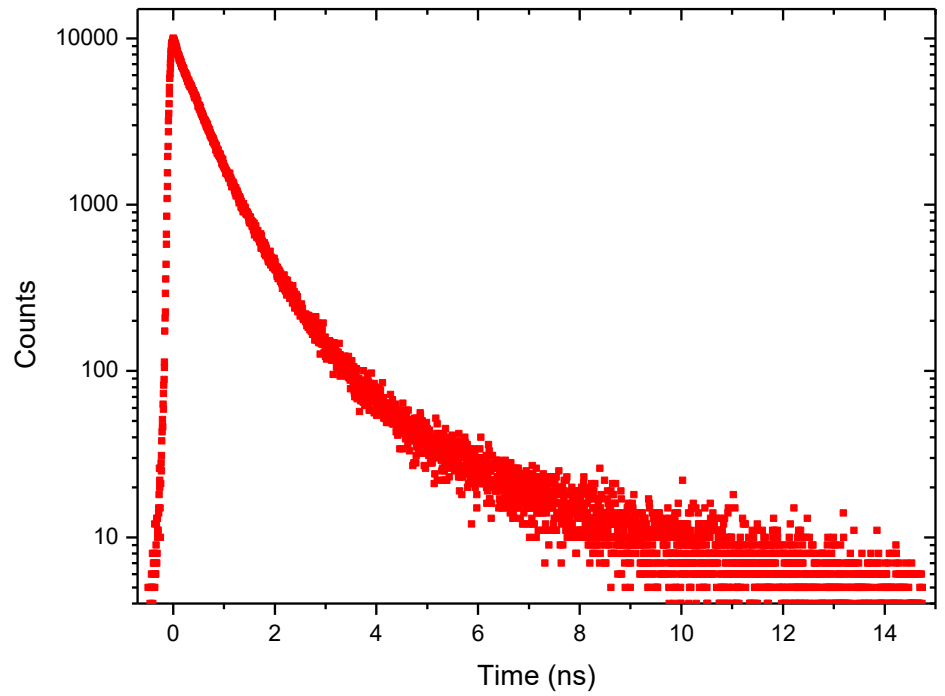

Figure S20: Fluorescence decay profile of $\mathbf{1 9}$ in the solid-state with excitation at $376 \mathrm{~nm}: \tau_{1}=0.554 \pm 0.001$ $(85.3 \%) \mathrm{ns} ; \tau_{2}=2.043 \pm 0.020(14.7 \%) \mathrm{ns} ; \chi^{2}=1.430$. 


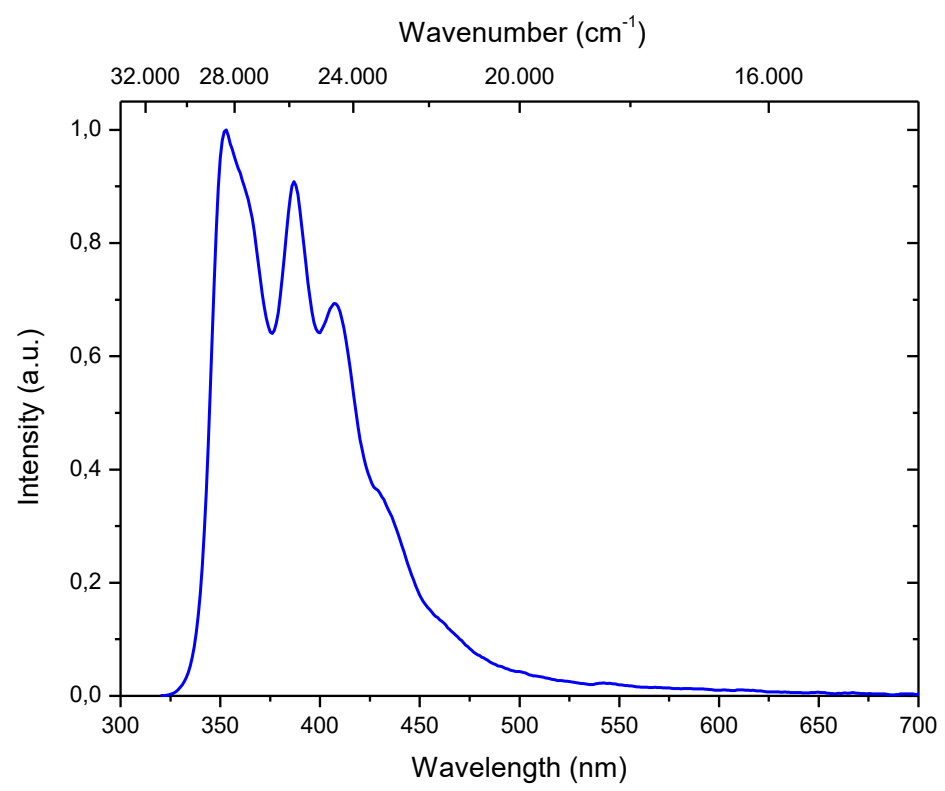

Figure S21: Normalized emission spectrum of $\mathbf{2 0}$ in the solid-state. Quantum yield in the solid-state was determined to $12.6 \%$.

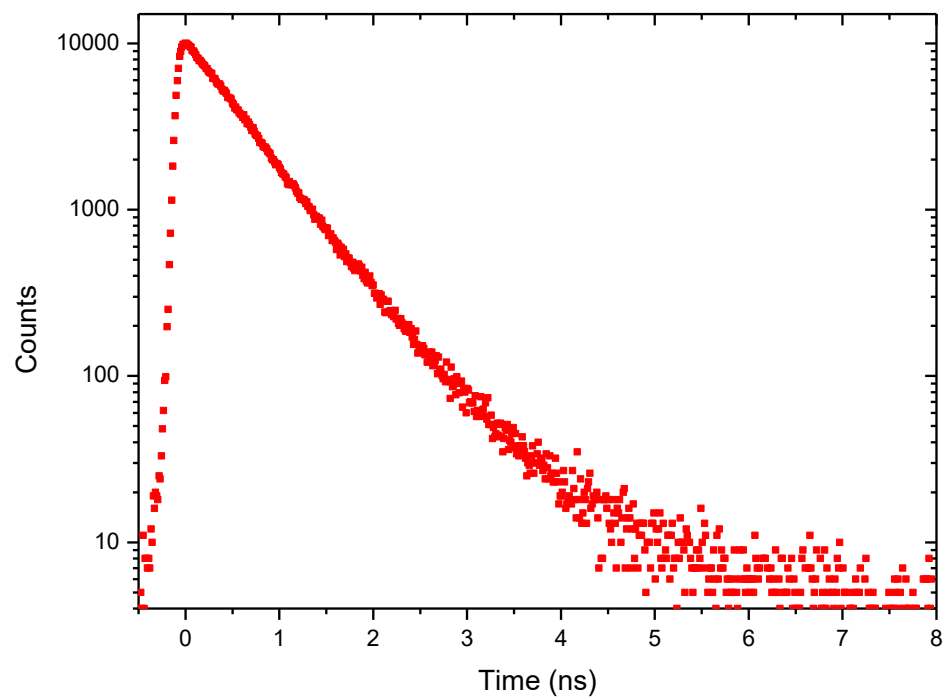

Figure S22: Fluorescence decay profile of 20 in the solid-state with excitation at $376 \mathrm{~nm}: \tau=0.587 \pm 0.001 \mathrm{~ns}$; $\chi^{2}=1.430$. Emission was detected at $407 \mathrm{~nm}$. 


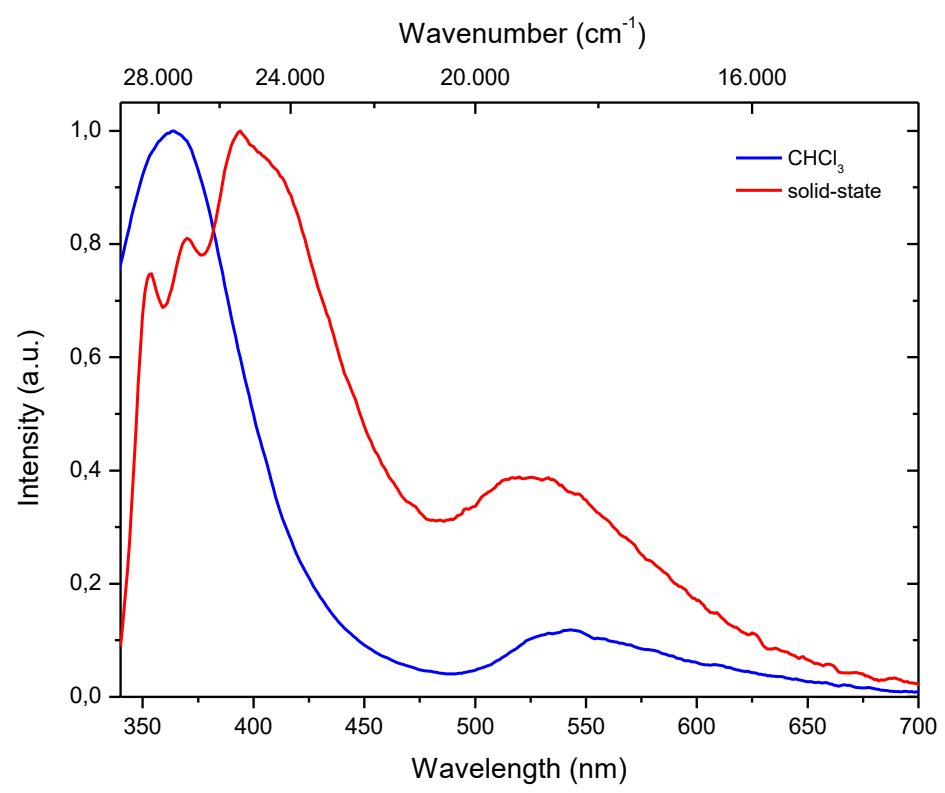

Figure S23: Normalized emission spectra of 21 in chloroform solution (blue line, $c=10^{-5} \mathrm{~mol} \mathrm{~L}^{-1}$ ) after bubbling with argon for 30 minutes and the solid-state (red line).

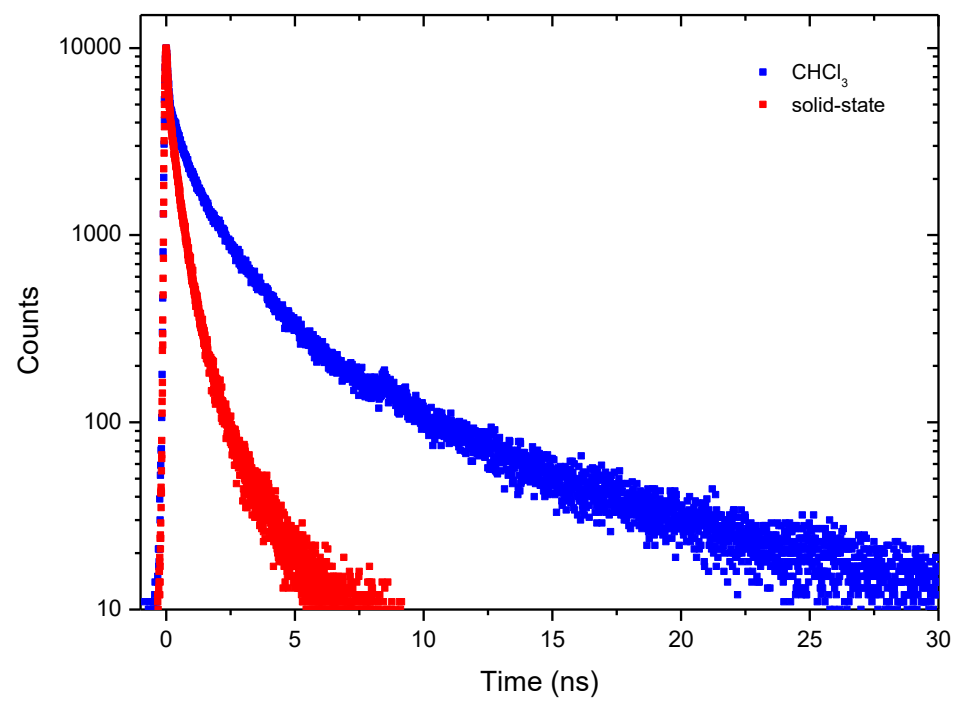

Figure S24: Fluorescence decay profile of 21 in chloroform solution (blue dots, detection at $400 \mathrm{~nm}$ ) after bubbling with argon for 30 minutes and the solid-state (red dots, detection at $392 \mathrm{~nm}$ ) with excitation at $376 \mathrm{~nm}$. $\mathrm{CHCl}_{3}: \tau_{1}=0.442 \pm 0.011(12.9 \%) \mathrm{ns} ; \tau_{2}=1.697 \pm 0.020(54.6 \%) \mathrm{ns} ; \tau_{3}=7.630 \pm 0.080(32.5 \%) \mathrm{ns} ; \chi^{2}=1.199$. Solid-state: $\tau_{1}=0.378 \pm 0.002(73.5 \%) \mathrm{ns} ; \tau_{2}=1.648 \pm 0.013(26.5 \%) \mathrm{ns} ; \chi^{2}=1.274$. 


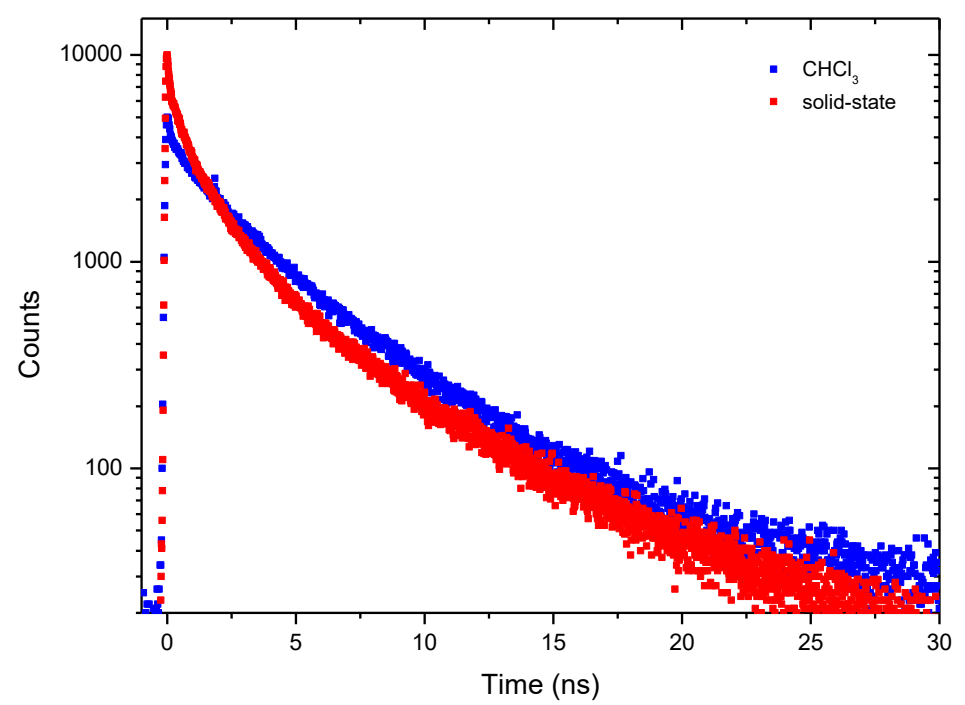

Figure S25: Fluorescence decay profile of 21 in chloroform solution (blue dots, detection at $550 \mathrm{~nm}$ ) after bubbling with argon for 30 minutes and the solid-state (red dots, detection at $527 \mathrm{~nm}$ ) with excitation at $376 \mathrm{~nm}$. $\mathrm{CHCl}_{3}: \tau_{1}=2.137 \pm 0.030(40.5 \%) \mathrm{ns} ; \tau_{2}=5.747 \pm 0.058(59.5 \%) \mathrm{ns} ; \chi^{2}=1.404$.

Solid-state: $\tau_{1}=1.503 \pm 0.008(45.2 \%) \mathrm{ns} ; \tau_{2}=6.153 \pm 0.027(54.8 \%) \mathrm{ns} ; \chi^{2}=1.445$.

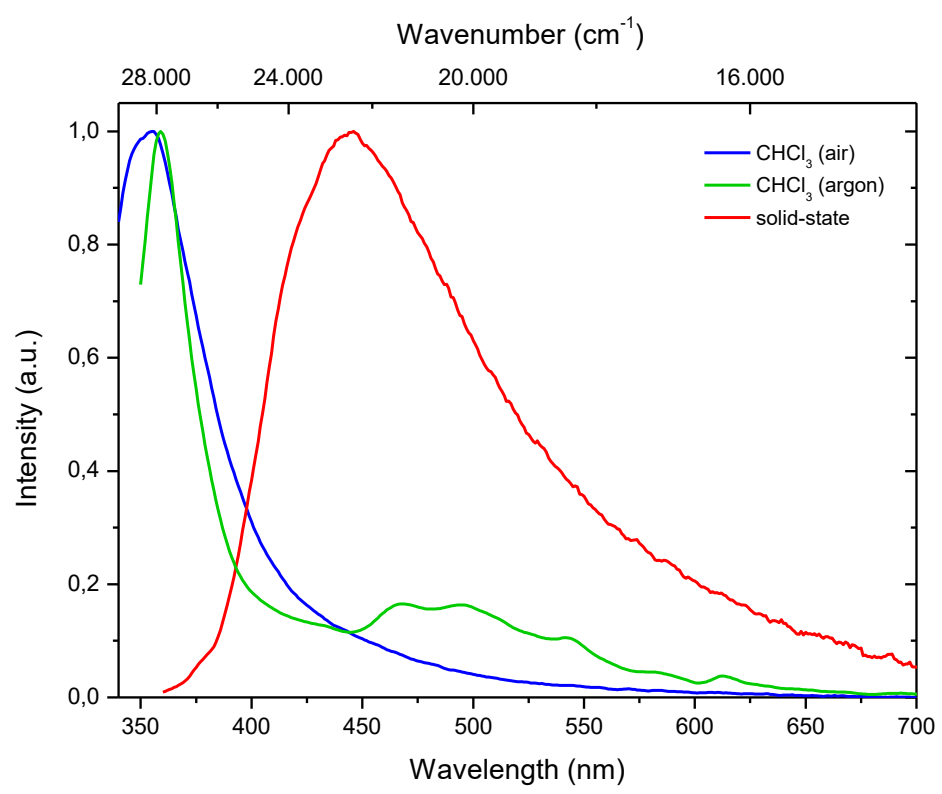

Figure S26: Normalized emission spectra of 5 in chloroform solution (blue line, $c=10^{-5}$ mol $\mathrm{L}^{-1}$ ) under air, in chloroform solution (green line, $c=10^{-5} \mathrm{~mol} \mathrm{~L}^{-1}$ ) after bubbling with argon for 30 minutes and the solid-state (red line). 


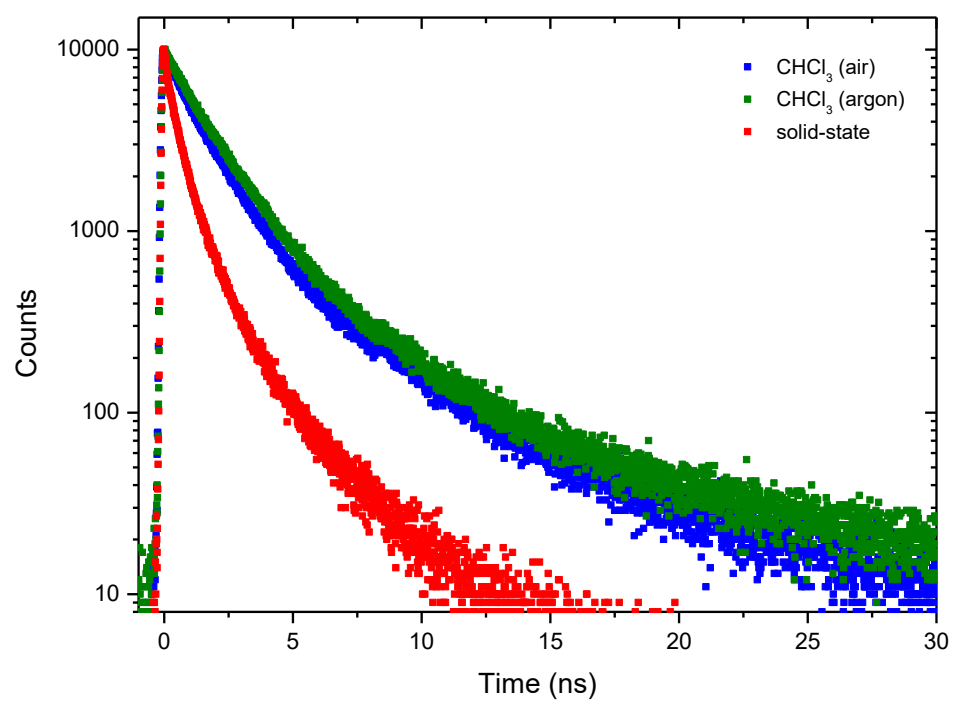

Figure S27: Fluorescence decay profile of 5 in chloroform solution under air (blue dots, detection at $450 \mathrm{~nm}$ ), in chloroform solution after bubbling with argon for 30 minutes (green dots, detection at $450 \mathrm{~nm}$ ) and the solid-state (red dots, detection at $446 \mathrm{~nm}$ ) with excitation at $376 \mathrm{~nm}$.

$\mathrm{CHCl}_{3}$ (air): $\tau_{1}=1.459 \pm 0.004(72.2 \%) \mathrm{ns} ; \tau_{2}=5.692 \pm 0.030(27.8 \%) \mathrm{ns} ; \chi^{2}=1.353$.

$\mathrm{CHCl}_{3}$ (argon): $\tau_{1}=1.709 \pm 0.004(77.1 \%) \mathrm{ns} ; \tau_{2}=6.288 \pm 0.045(22.9 \%) \mathrm{ns} ; \chi^{2}=1.265$.

Solid-state: $\tau_{1}=0.637 \pm 0.003(66.2 \%) \mathrm{ns} ; \tau_{2}=2.601 \pm 0.015(33.8 \%) \mathrm{ns} ; \chi^{2}=1.183$.

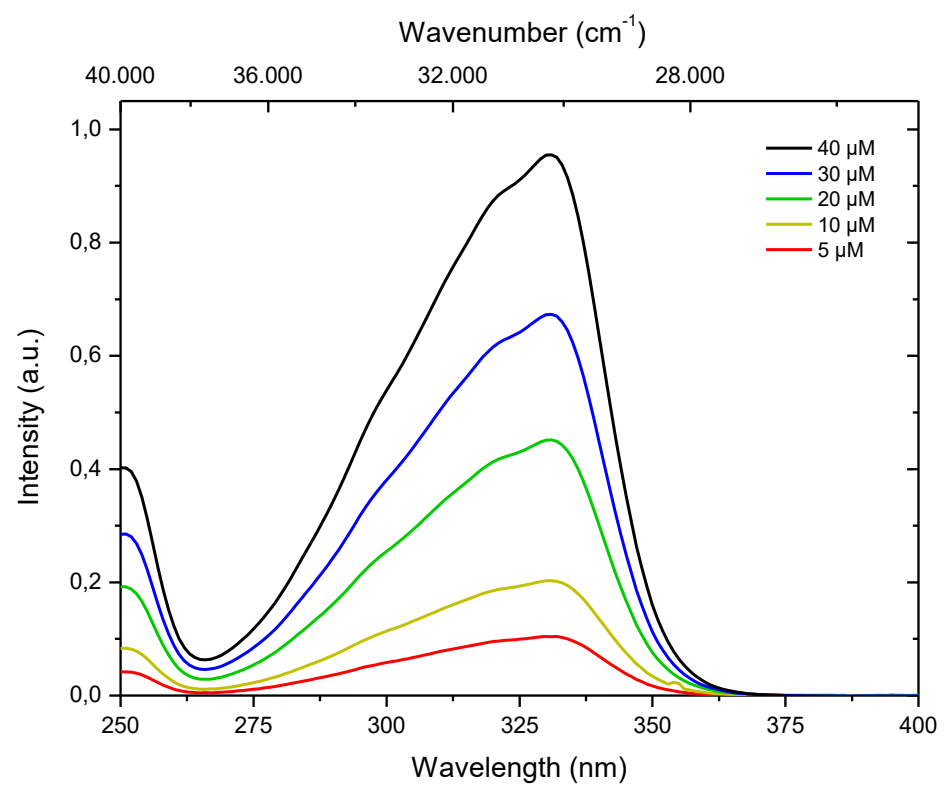

Figure S28: Absorption spectra of $\mathbf{5}$ at various concentration in chloroform solution. 


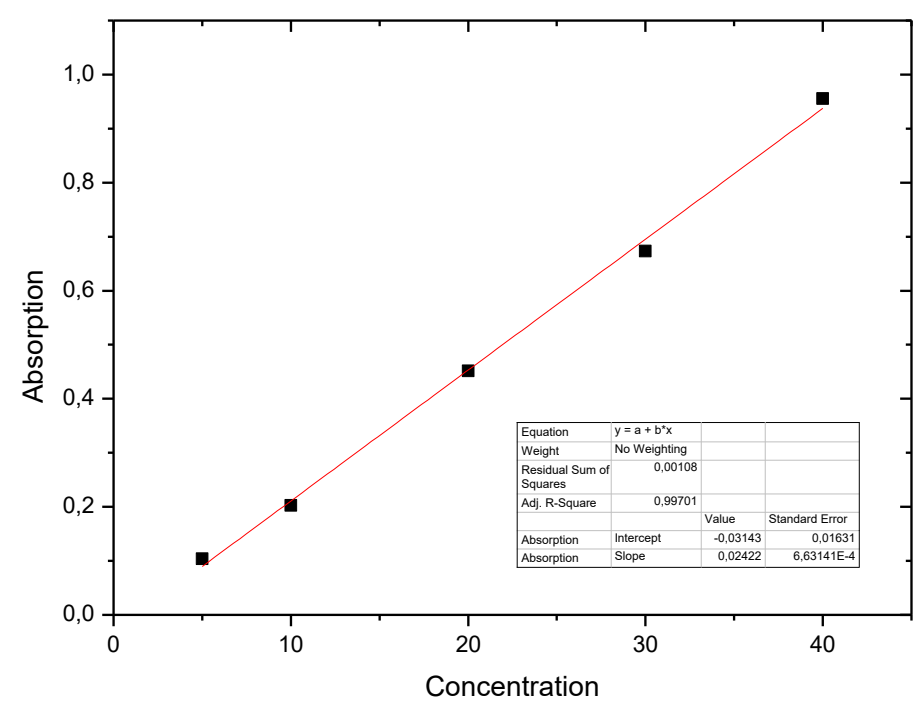

Figure S29: Linear regression for extinction coefficient determination (with respect to Figure S28): $\varepsilon_{331}=24.220$ $\mathrm{L} \mathrm{mol}^{-1} \mathrm{~cm}^{-1}$.

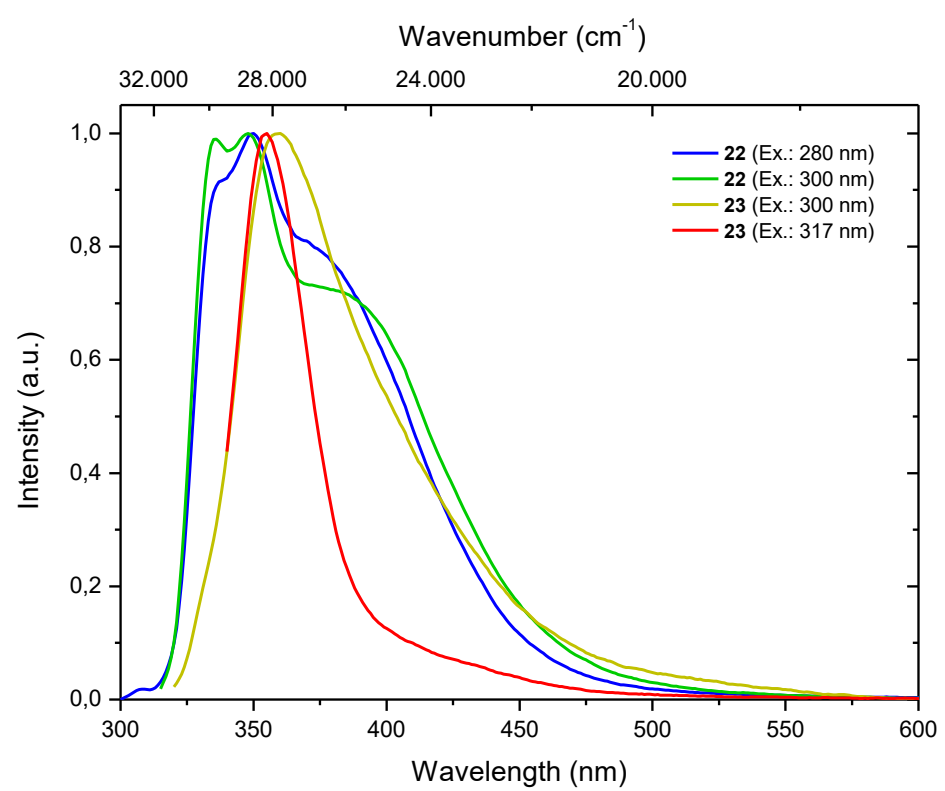

Figure S30: Normalized emission spectra of 22 in chloroform solution (blue line, $c=10^{-5} \mathrm{~mol} \mathrm{~L}^{-1}$, excitation at $280 \mathrm{~nm}$ ) and (green line, excitation at $300 \mathrm{~nm}$ ) and of $\mathbf{2 3}$ in chloroform solution (yellow line, $c=10^{-5} \mathrm{~mol} \mathrm{~L}^{-1}$, excitation at $300 \mathrm{~nm}$ ) and (red line, excitation at $317 \mathrm{~nm}$ ) after bubbling with argon for 30 minutes. 


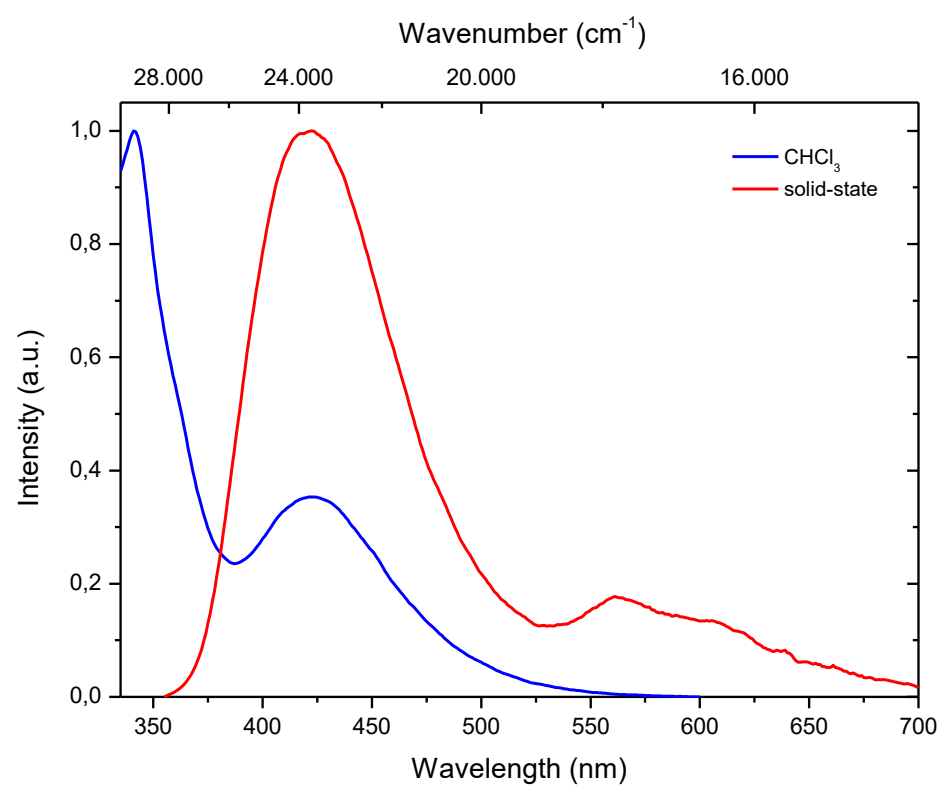

Figure S31: Normalized emission spectra of 25 in chloroform solution (blue line, $c=10^{-5} \mathrm{~mol} \mathrm{~L}^{-1}$ ) after bubbling with argon for 30 minutes and the solid-state (red line).

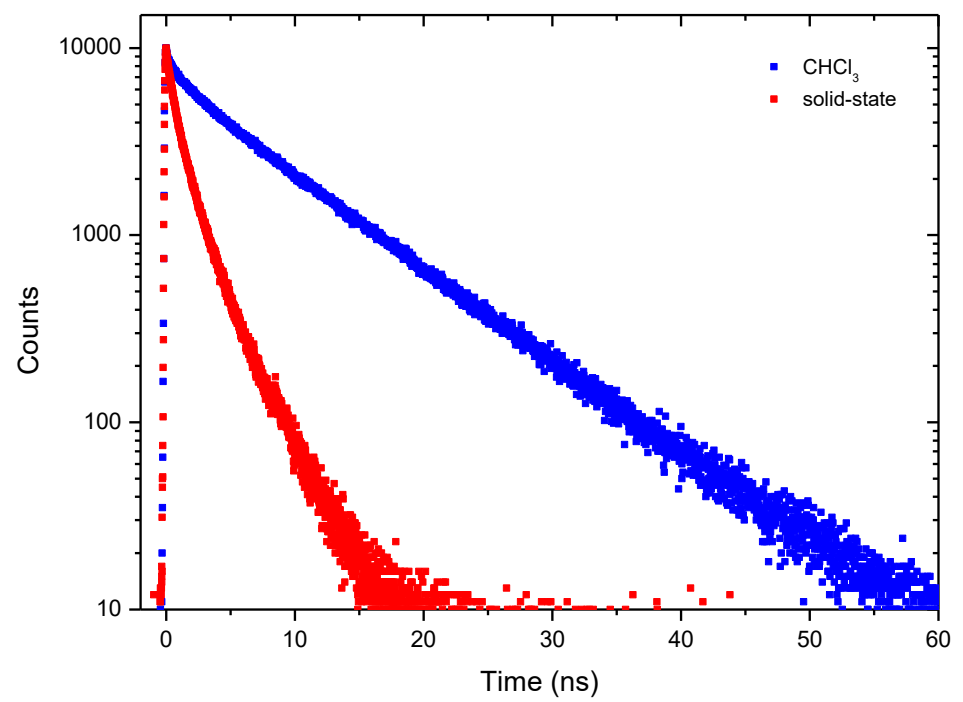

Figure S32: Fluorescence decay profile of $\mathbf{2 5}$ in chloroform solution (blue dots, detection at $430 \mathrm{~nm}$ ) after bubbling with argon for 30 minutes and the solid-state (red dots, detection at $560 \mathrm{~nm}$ ) with excitation at $376 \mathrm{~nm}$. $\mathrm{CHCl}_{3}: \tau_{1}=1.505 \pm 0.027(5.5 \%) \mathrm{ns} ; \tau_{2}=8.661 \pm 0.009$ (94.5\%) ns; $\chi^{2}=1.135$.

Solid-state: $\tau_{1}=0.876 \pm 0.005(44.9 \%) \mathrm{ns} ; \tau_{2}=2.826 \pm 0.010(55.1 \%) \mathrm{ns} ; \chi^{2}=1.404$. 


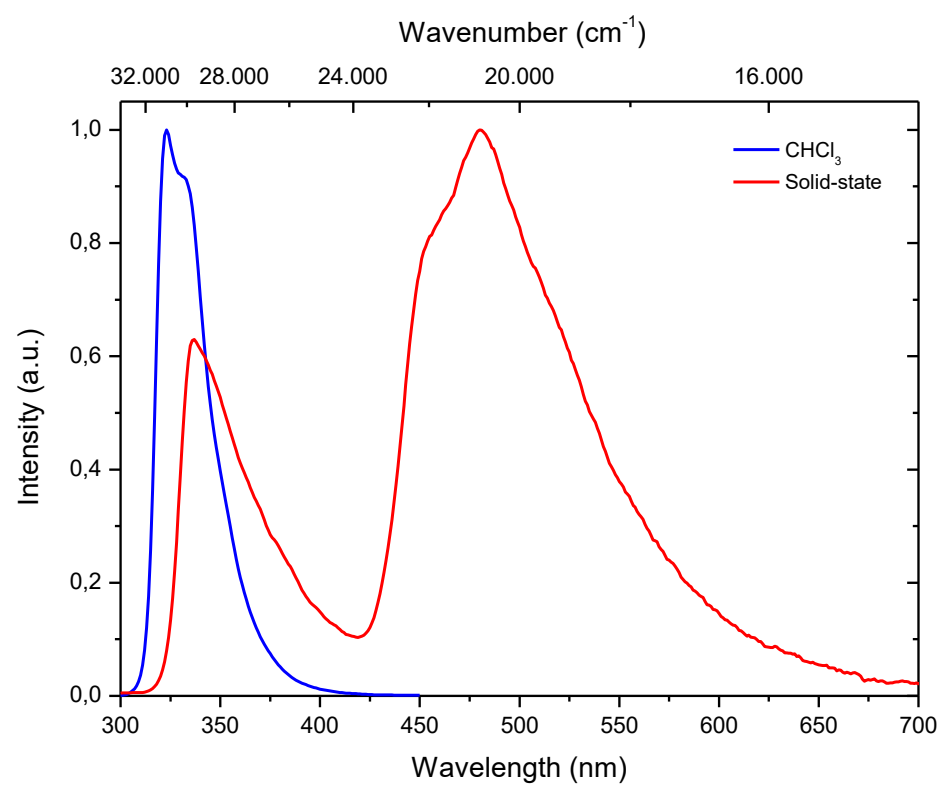

Figure S33: Normalized emission spectra of $\mathbf{2 8}$ in chloroform solution (blue line, $c=10^{-5} \mathrm{~mol} \mathrm{~L}^{-1}$ ) after bubbling with argon for 30 minutes and the solid-state (red line).

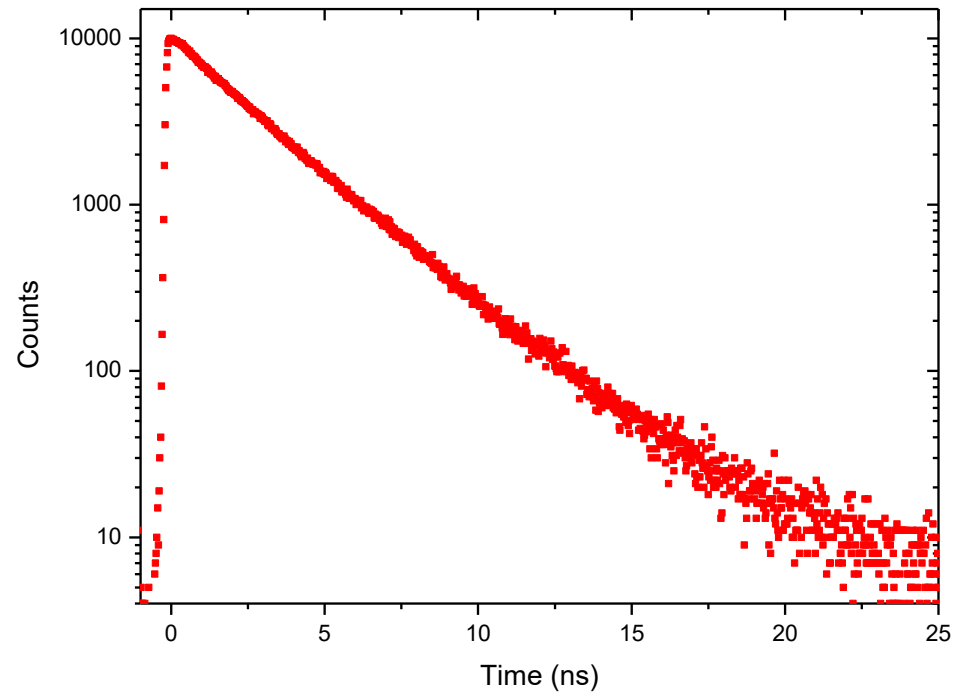

Figure S34: Fluorescence decay profile of 28 in the solid-state with excitation at $376 \mathrm{~nm}: \tau_{1}=2.564 \pm 0.009 \mathrm{~ns}$ $(93.0 \%) ; \tau_{2}=5.513 \pm 0.158(7.1 \%) \mathrm{ns} ; \chi^{2}=1.099$. Emission was detected at $480 \mathrm{~nm}$. 


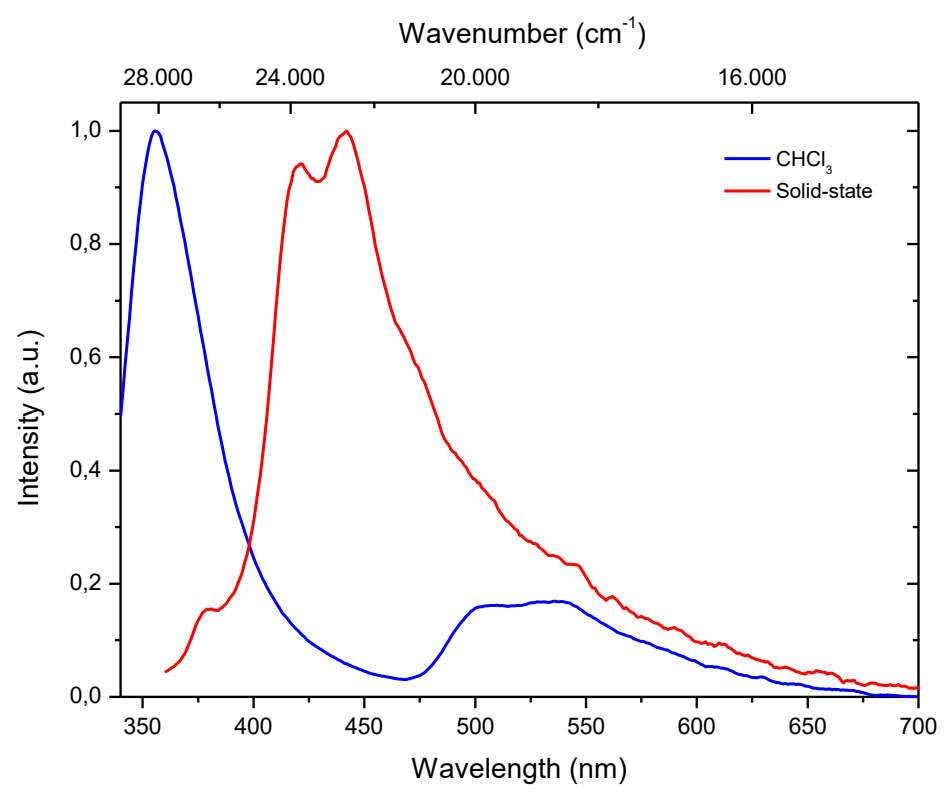

Figure S35: Normalized emission spectra of $\mathbf{4 2}$ in chloroform solution (blue line, $c=10^{-5} \mathrm{~mol} \mathrm{~L}^{-1}$ ) after bubbling with argon for 30 minutes and the solid-state (red line).

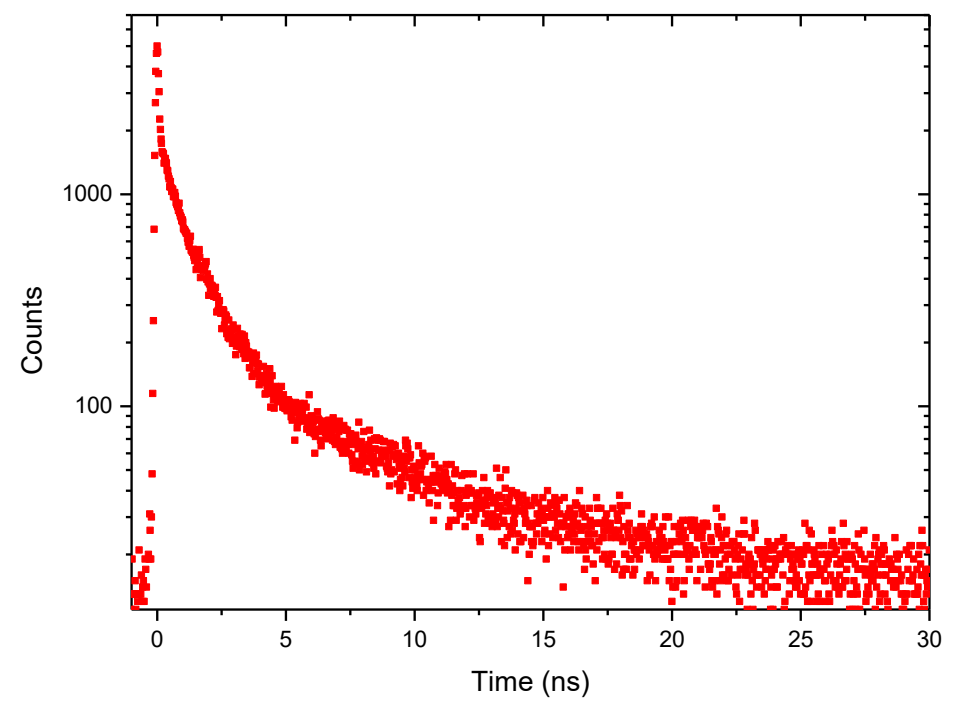

Figure S36: Fluorescence decay profile of 42 in chloroform solution $\left(c=10^{-5} \mathrm{~mol} \mathrm{~L}^{-1}\right)$ after bubbling with argon for 30 minutes with excitation at $376 \mathrm{~nm}: \tau_{1}=1.004 \pm 0.010(57.7 \%) \mathrm{ns} ; \tau_{2}=6.171 \pm 0.104(42.3 \%) \mathrm{ns} ; \chi^{2}=1.285$. Emission was detected at $540 \mathrm{~nm}$. 


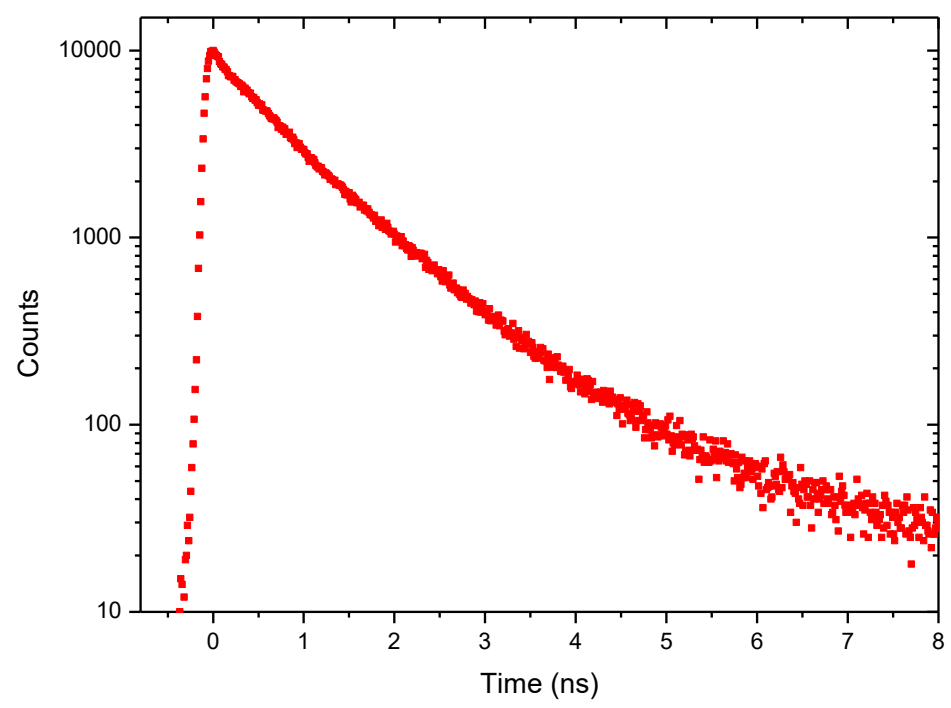

Figure S37: Fluorescence decay profile of $\mathbf{4 2}$ in the solid-state with excitation at $376 \mathrm{~nm}: \tau_{1}=0.871 \pm 0.002 \mathrm{~ns}$ $(90.7 \%) ; \tau_{2}=4.805 \pm 0.049(9.3 \%) \mathrm{ns} ; \chi^{2}=1.457$. Emission was detected at $442 \mathrm{~nm}$.

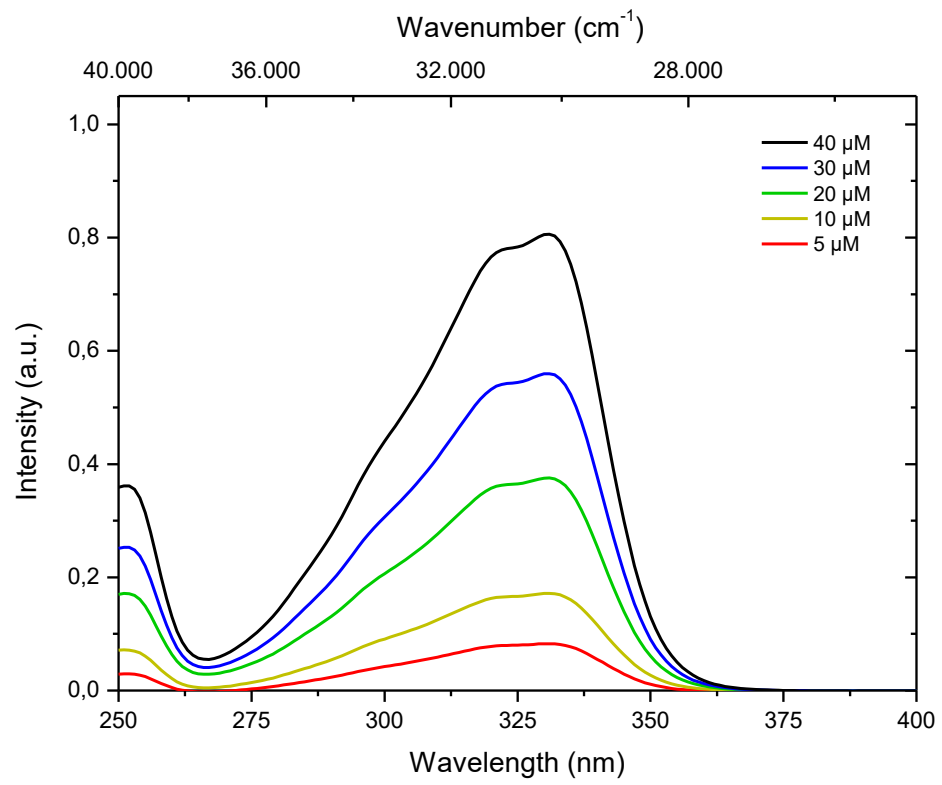

Figure S38: Absorption spectra of $\mathbf{4 2}$ at various concentration in chloroform solution. 


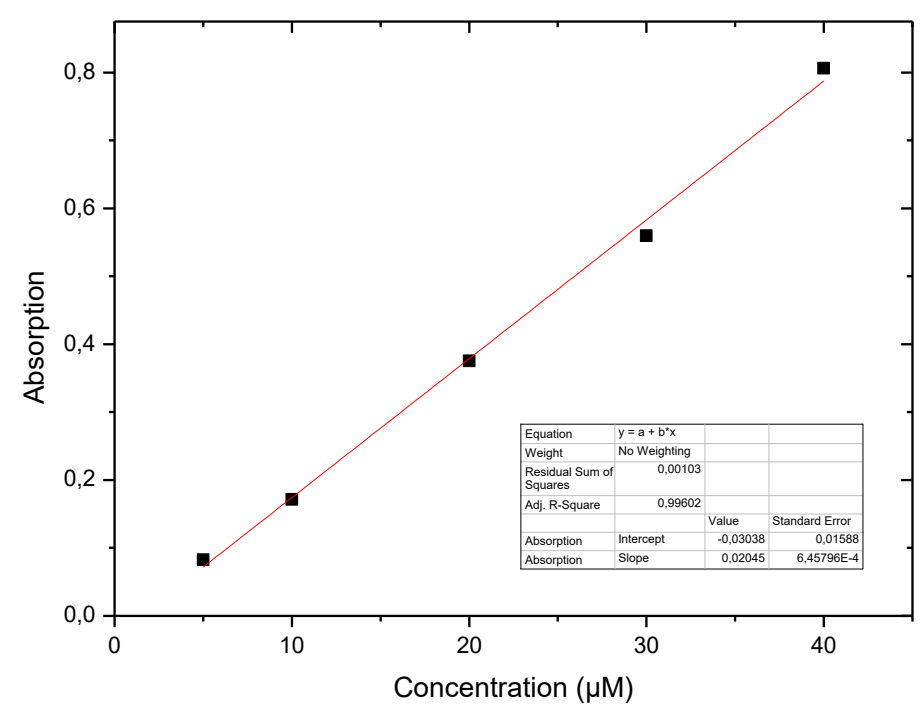

Figure S39: Linear regression for extinction coefficient determination (with respect to Figure S38): $\varepsilon_{331}=20.450$ $\mathrm{L} \mathrm{mol}^{-1} \mathrm{~cm}^{-1}$.

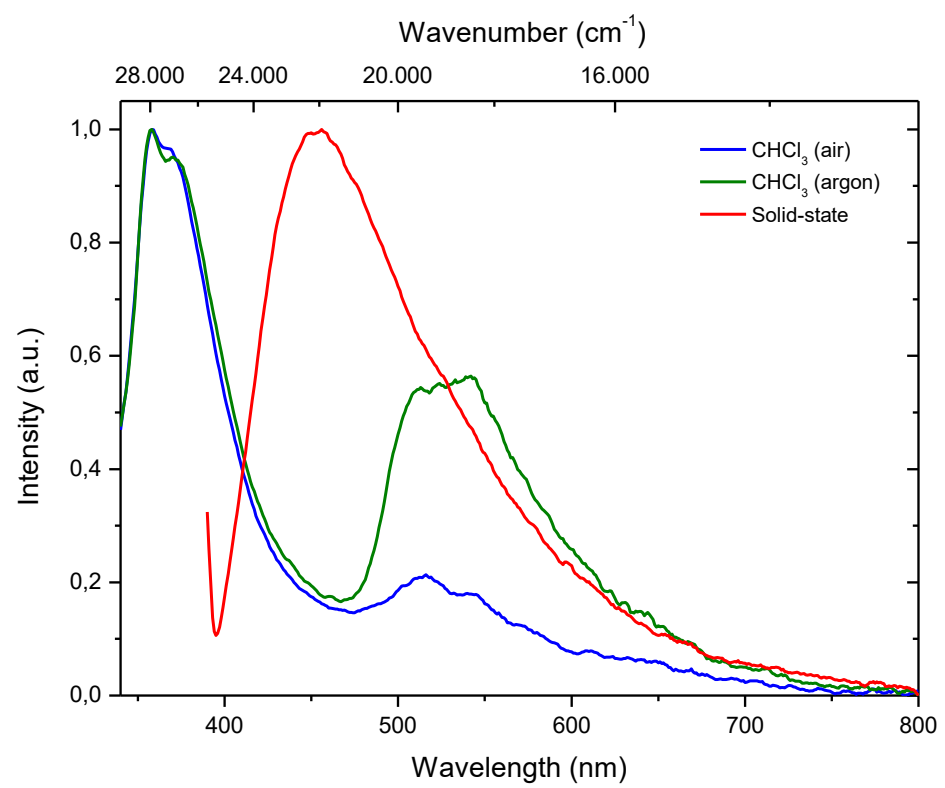

Figure S40: Normalized emission spectra of 45 in chloroform solution (blue line, $c=10^{-5} \mathrm{~mol} \mathrm{~L}^{-1}$ ) under air, in chloroform solution (green line, $c=10^{-5} \mathrm{~mol} \mathrm{~L}^{-1}$ ) after bubbling with argon for 30 minutes and the solid-state (red line). 


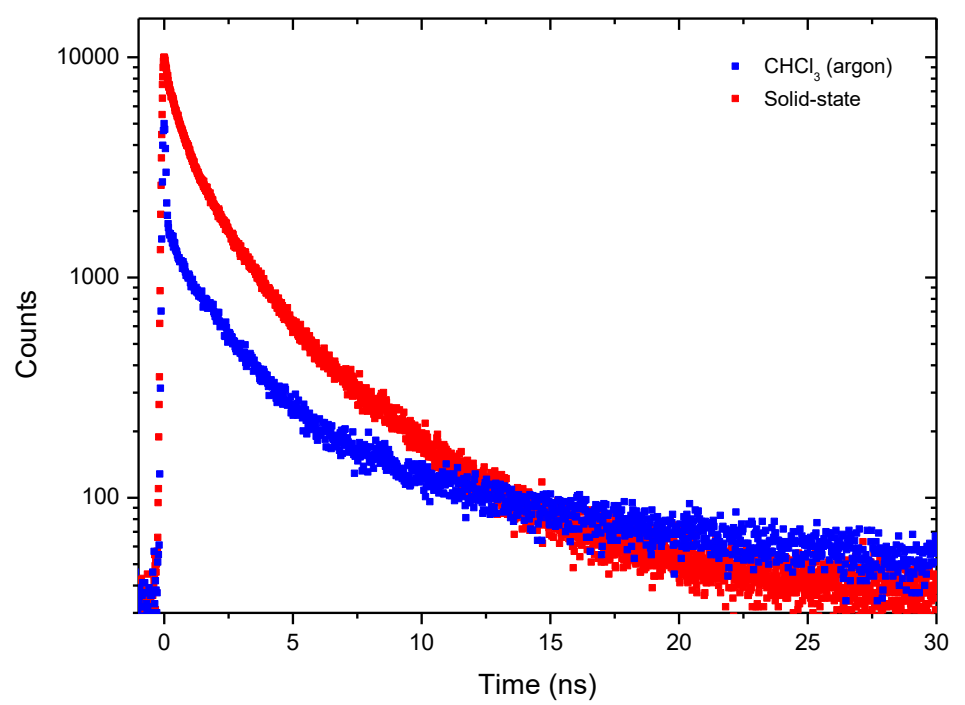

Figure S41: Fluorescence decay profile of $\mathbf{4 5}$ in chloroform solution (blue dots, detection at $540 \mathrm{~nm}$ ) after bubbling with argon for 30 minutes and the solid-state (red dots, detection at $580 \mathrm{~nm}$ ) with excitation at $376 \mathrm{~nm}$. $\mathrm{CHCl}_{3}: \tau_{1}=1.824 \pm 0.018(51.3 \%) \mathrm{ns} ; \tau_{2}=11.56 \pm 0.31(48.7 \%) \mathrm{ns} ; \chi^{2}=1.225$.

Solid-state: $\tau_{1}=1.076 \pm 0.006(43.9 \%) \mathrm{ns} ; \tau_{2}=4.050 \pm 0.018(56.1 \%) \mathrm{ns} ; \chi^{2}=1.435$.

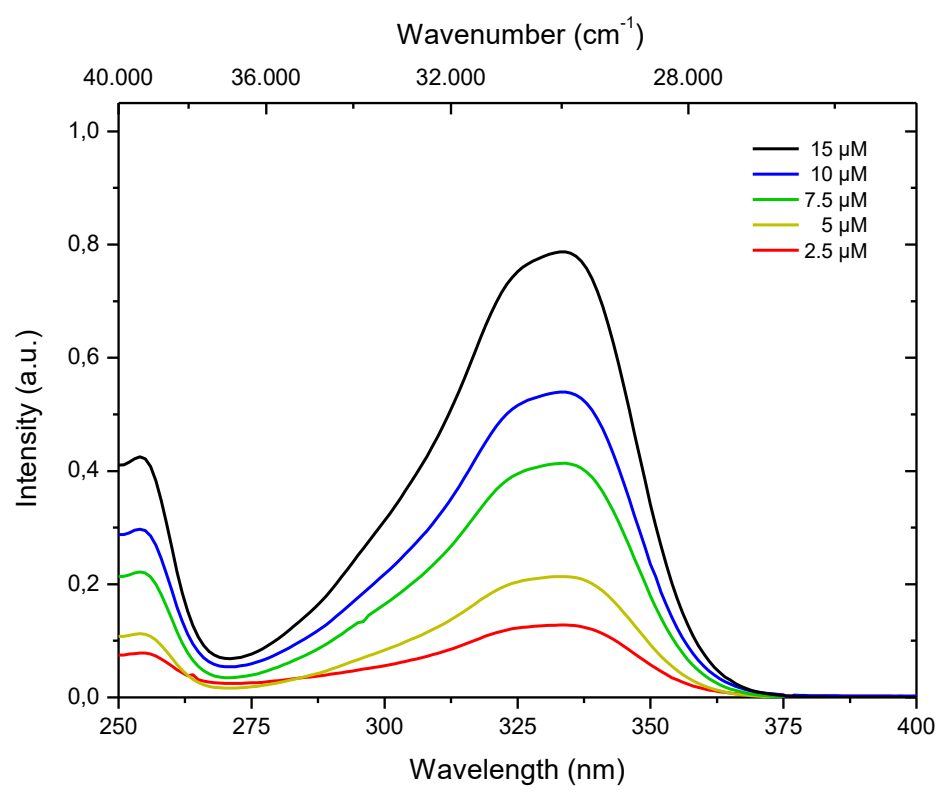

Figure S42: Absorption spectra of $\mathbf{4 5}$ at various concentration in chloroform solution. 


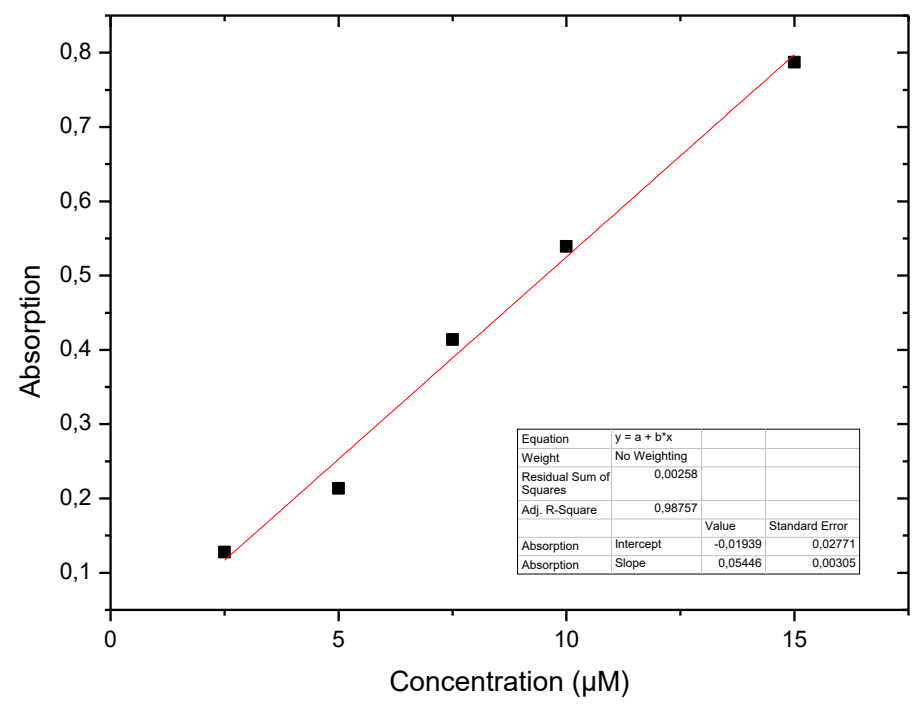

Figure S43: Linear regression for extinction coefficient determination (with respect to Figure S42): $\varepsilon_{334}=54.460$ $\mathrm{L} \mathrm{mol}^{-1} \mathrm{~cm}^{-1}$. 


\section{Crystal Structure of 3-(Azidomethyl)-7-bromo-9,9-dimethyl- $9 H$-fluorene-2-carbaldehyde (5)}

\subsection{Crystal Data for (5)}

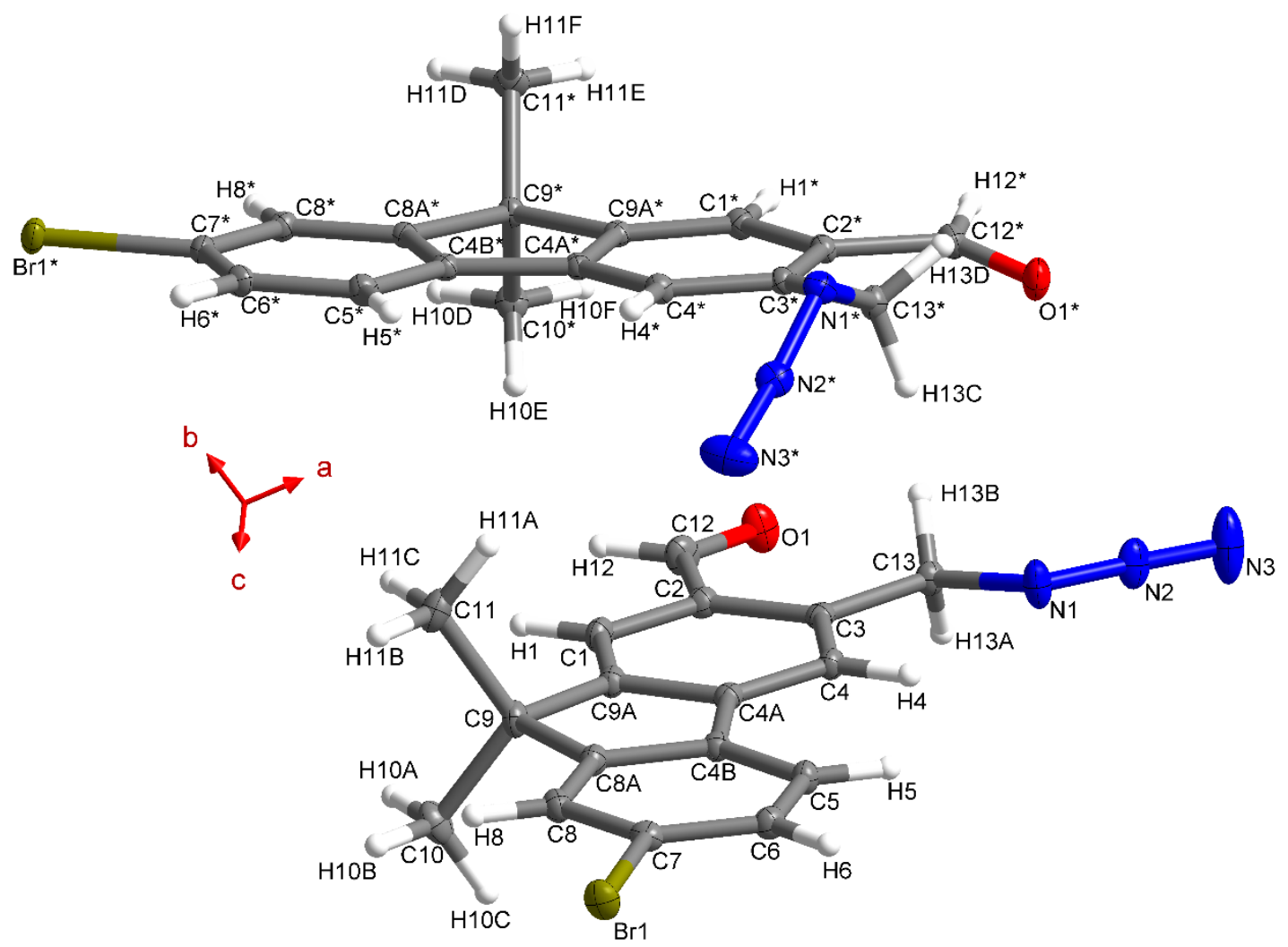

Figure S1: Asymmetric unit of $\mathbf{5}$ with atom labels.

Table S2: Crystal data and structure refinement for 5.

\begin{tabular}{ll}
\hline Empirical formula & $\mathrm{C}_{17} \mathrm{H}_{14} \mathrm{BrN}_{3} \mathrm{O}$ \\
Formula weight, $\mathrm{g} \mathrm{mol}^{-1}$ & 356.22 \\
Crystal system & Triclinic \\
Crystal size, $\mathrm{mm}^{3}$ & $0.3 \times 0.2 \times 0.2$ \\
Space group (number) & $\mathrm{P}-1(2)$ \\
$\mathrm{a} / \AA$ & $8.2141(3)$ \\
$\mathrm{b} / \AA$ & $12.7921(4)$ \\
$\mathrm{c} / \AA$ & $16.1129(6)$ \\
$\alpha{ }^{\circ}$ & $107.8710(10)$ \\
$\beta /^{\circ}$ & $102.8730(10)$ \\
$\gamma /{ }^{\circ}$ & $99.2410(10)$ \\
Volume $/ \AA^{3}$ & $1522.30(9)$
\end{tabular}


Z

$\rho_{\text {calc, }} \mathrm{g} / \mathrm{cm}^{3}$

$\mu / \mathrm{mm}^{-1}$

$F(000)$

$2 \theta$ range for data collection $/^{\circ}$

Index ranges

No. of reflections collected

No. of independent reflections

Data/restraints/parameters

Goodness-of-fit on $\mathrm{F}^{2}$

Final $\mathrm{R}$ indexes $[\mathrm{I} \geq 2 \sigma(\mathrm{I})]$

Final $\mathrm{R}$ indexes [all data]

Largest diff. peak/hole/ e $\AA^{-3}$

Completeness to $\theta=25.242^{\circ}$

CCDC number
4

1.554

2.705

720

5.18 to 61.22

$-11 \leq \mathrm{h} \leq 11$

$-18 \leq \mathrm{k} \leq 18$

$-23 \leq 1 \leq 23$

53013

$9309\left[R_{\text {int }}=0.0274, R_{\text {sigma }}=0.0219\right]$

9309/0/401

1.081

$\mathrm{R}_{1}=0.0246, \mathrm{wR}_{2}=0.0540$

$\mathrm{R}_{1}=0.0310, \mathrm{wR}_{2}=0.0561$

$0.60 /-0.46$

$99.9 \%$

1989134

Table S3: Fractional Atomic Coordinates $\left[\mathrm{A}^{2}\right]$ and Equivalent Isotropic Displacement Parameters $\left[\AA^{2}\right]$ for 5. $\mathrm{U}_{\mathrm{eq}}$ is defined as $1 / 3$ of the trace of the orthogonalised $\mathrm{U}_{\mathrm{IJ}}$ tensor.

\begin{tabular}{ccccc}
\hline Atom & $\boldsymbol{x}$ & $\boldsymbol{y}$ & $\boldsymbol{z}$ & $\boldsymbol{U}_{\text {eq }}$ \\
\hline $\mathrm{Br} 1$ & $0.62959(2)$ & $0.87935(2)$ & $0.88875(2)$ & $0.01738(4)$ \\
$\mathrm{O} 1$ & $0.33038(13)$ & $0.46964(9)$ & $0.13714(7)$ & $0.0220(2)$ \\
$\mathrm{N} 1$ & $0.79293(15)$ & $0.48548(11)$ & $0.32343(8)$ & $0.0190(2)$ \\
$\mathrm{N} 2$ & $0.87272(16)$ & $0.42834(11)$ & $0.27937(8)$ & $0.0204(2)$ \\
$\mathrm{N} 3$ & $0.95945(19)$ & $0.37986(15)$ & $0.24644(10)$ & $0.0381(4)$ \\
$\mathrm{C} 1$ & $0.28731(16)$ & $0.63068(11)$ & $0.35404(9)$ & $0.0144(2)$ \\
$\mathrm{H} 1$ & 0.181517 & 0.646152 & 0.329310 & 0.017 \\
$\mathrm{C} 2$ & $0.36981(16)$ & $0.56857(11)$ & $0.29586(9)$ & $0.0132(2)$ \\
$\mathrm{C} 3$ & $0.52889(16)$ & $0.54570(10)$ & $0.33167(8)$ & $0.0113(2)$ \\
$\mathrm{C} 4$ & $0.59980(16)$ & $0.58406(10)$ & $0.42580(8)$ & $0.0117(2)$ \\
$\mathrm{H} 4$ & 0.705325 & 0.568757 & 0.451174 & 0.014 \\
$\mathrm{C} 4 \mathrm{~A}$ & $0.51590(16)$ & $0.64483(10)$ & $0.48263(8)$ & $0.0115(2)$ \\
$\mathrm{C} 4 \mathrm{~B}$ & $0.56563(16)$ & $0.69502(10)$ & $0.58212(8)$ & $0.0115(2)$ \\
$\mathrm{C} 5$ & $0.70807(16)$ & $0.69389(11)$ & $0.64733(9)$ & $0.0134(2)$ \\
$\mathrm{H} 5$ & 0.791526 & 0.655539 & 0.629495 & 0.016 \\
$\mathrm{C} 6$ & $0.72612(17)$ & $0.74983(11)$ & $0.73898(9)$ & $0.0136(2)$
\end{tabular}




\begin{tabular}{|c|c|c|c|c|}
\hline H6 & 0.822249 & 0.750274 & 0.784673 & 0.016 \\
\hline $\mathrm{C} 7$ & $0.60132(16)$ & $0.80525(11)$ & $0.76293(9)$ & $0.0127(2)$ \\
\hline $\mathrm{C} 8$ & $0.45795(16)$ & $0.80749(11)$ & $0.69884(9)$ & $0.0138(2)$ \\
\hline H8 & 0.374611 & 0.845824 & 0.716874 & 0.017 \\
\hline C8A & $0.44162(16)$ & $0.75146(11)$ & $0.60754(9)$ & $0.0123(2)$ \\
\hline C9 & $0.29979(16)$ & $0.74108(11)$ & $0.52397(9)$ & $0.0137(2)$ \\
\hline C9A & $0.36006(16)$ & $0.66938(11)$ & $0.44743(9)$ & $0.0124(2)$ \\
\hline $\mathrm{C} 10$ & $0.12230(17)$ & $0.68180(13)$ & $0.52496(10)$ & $0.0200(3)$ \\
\hline H10A & 0.036244 & 0.669237 & 0.467621 & 0.030 \\
\hline H10B & 0.090161 & 0.729581 & 0.576028 & 0.030 \\
\hline $\mathrm{H} 10 \mathrm{C}$ & 0.127354 & 0.608788 & 0.531852 & 0.030 \\
\hline $\mathrm{C} 11$ & $0.29608(19)$ & $0.85823(12)$ & $0.51709(10)$ & $0.0197(3)$ \\
\hline H11A & 0.410171 & 0.895712 & 0.517395 & 0.030 \\
\hline H11B & 0.264871 & 0.904650 & 0.569130 & 0.030 \\
\hline $\mathrm{H} 11 \mathrm{C}$ & 0.210823 & 0.848851 & 0.460379 & 0.030 \\
\hline $\mathrm{C} 12$ & $0.28224(17)$ & $0.52849(12)$ & $0.19717(9)$ & $0.0178(3)$ \\
\hline $\mathrm{H} 12$ & 0.179054 & 0.550673 & 0.178968 & 0.021 \\
\hline $\mathrm{C} 13$ & $0.61929(16)$ & $0.48143(11)$ & $0.26895(8)$ & $0.0129(2)$ \\
\hline H13A & 0.551907 & 0.401722 & 0.237200 & 0.016 \\
\hline H13B & 0.629690 & 0.515846 & 0.222492 & 0.016 \\
\hline $\mathrm{Br} 1^{*}$ & $-0.22890(2)$ & $1.00015(2)$ & $0.14743(2)$ & $0.01550(4)$ \\
\hline $\mathrm{O} 1 *$ & $0.89725(13)$ & $0.61783(9)$ & $0.17610(7)$ & $0.0191(2)$ \\
\hline $\mathrm{N} 1 *$ & $0.88357(14)$ & $0.89128(9)$ & $0.40051(8)$ & $0.0143(2)$ \\
\hline $\mathrm{N} 2 *$ & $0.81665(15)$ & $0.88222(10)$ & $0.46022(8)$ & $0.0158(2)$ \\
\hline N3* & $0.76277(19)$ & $0.88614(12)$ & $0.51975(9)$ & $0.0285(3)$ \\
\hline $\mathrm{C} 1 *$ & $0.51351(16)$ & $0.69391(11)$ & $0.08530(9)$ & $0.0121(2)$ \\
\hline $\mathrm{H} 1 *$ & 0.499974 & 0.649222 & 0.023855 & 0.015 \\
\hline $\mathrm{C} 2 *$ & $0.65942(16)$ & $0.70333(11)$ & $0.15443(9)$ & $0.0117(2)$ \\
\hline C $3 *$ & $0.68417(16)$ & $0.77256(10)$ & $0.24610(8)$ & $0.0111(2)$ \\
\hline $\mathrm{C} 4 *$ & $0.55942(16)$ & $0.82955(11)$ & $0.26659(8)$ & $0.0112(2)$ \\
\hline $\mathrm{H} 4 *$ & 0.573964 & 0.876650 & 0.327514 & 0.013 \\
\hline C4A* & $0.41258(15)$ & $0.81752(10)$ & $0.19750(8)$ & $0.0100(2)$ \\
\hline C4B* & $0.26634(16)$ & $0.86909(10)$ & $0.20113(8)$ & $0.0106(2)$ \\
\hline $\mathrm{C} 5 *$ & $0.23047(16)$ & $0.94449(11)$ & $0.27376(8)$ & $0.0121(2)$ \\
\hline $\mathrm{H} 5 *$ & 0.306336 & 0.969258 & 0.333719 & 0.015 \\
\hline C6* & $0.08156(17)$ & $0.98299(11)$ & $0.25707(9)$ & $0.0129(2)$ \\
\hline $\mathrm{H} 6^{*}$ & 0.054806 & 1.034677 & 0.305587 & 0.016 \\
\hline
\end{tabular}




$\begin{array}{ccccc}\text { C7* } & -0.02773(16) & 0.94503(11) & 0.16862(9) & 0.0120(2) \\ \text { C8* } & 0.00514(16) & 0.86908(11) & 0.09497(9) & 0.0121(2) \\ \text { H8* } & -0.071691 & 0.843831 & 0.035221 & 0.014 \\ \text { C8A* } & 0.15437(15) & 0.83179(10) & 0.11232(8) & 0.0102(2) \\ \text { C9* } & 0.22183(16) & 0.75194(10) & 0.04453(8) & 0.0105(2) \\ \text { C9A* } & 0.38937(15) & 0.74993(10) & 0.10694(8) & 0.0102(2) \\ \text { C10* } & 0.09774(16) & 0.63361(11) & -0.00032(9) & 0.0142(2) \\ \text { H10D } & -0.013154 & 0.638890 & -0.034819 & 0.021 \\ \text { H10E } & 0.080477 & 0.603261 & 0.046861 & 0.021 \\ \text { H10F } & 0.146688 & 0.583159 & -0.041645 & 0.021 \\ \text { C11* } & 0.25559(18) & 0.79907(12) & -0.02848(9) & 0.0156(2) \\ \text { H11D } & 0.146586 & 0.804420 & -0.064864 & 0.023 \\ \text { H11E } & 0.306879 & 0.748393 & -0.068189 & 0.023 \\ \text { H11F } & 0.334881 & 0.874536 & 0.000948 & 0.023 \\ \text { C12* } & 0.78038(17) & 0.63657(11) & 0.12570(9) & 0.0148(2) \\ \text { H12* } & 0.766049 & 0.605275 & 0.062135 & 0.018 \\ \text { C13* } & 0.84582(16) & 0.78619(11) & 0.31997(8) & 0.0128(2) \\ \text { H13C } & 0.832009 & 0.719942 & 0.339569 & 0.015 \\ \text { H13D } & 0.944912 & 0.787400 & 0.294493 & 0.015\end{array}$

Table S4: Bond Lengths for 5.

\begin{tabular}{cccc}
\hline Atom-Atom & Length $[\AA]$ & Atom-Atom & Length $[\AA]$ \\
\hline $\mathrm{Br} 1-\mathrm{C} 7$ & $1.8980(13)$ & $\mathrm{Br} 1^{*}-\mathrm{C} 7^{*}$ & $1.9013(12)$ \\
$\mathrm{O} 1-\mathrm{C} 12$ & $1.2157(17)$ & $\mathrm{O} 1^{*}-\mathrm{C} 12^{*}$ & $1.2185(16)$ \\
$\mathrm{N} 1-\mathrm{N} 2$ & $1.2291(16)$ & $\mathrm{N} 1^{*}-\mathrm{N} 2^{*}$ & $1.2349(16)$ \\
$\mathrm{N} 1-\mathrm{C} 13$ & $1.4826(16)$ & $\mathrm{N} 1^{*}-\mathrm{C} 13^{*}$ & $1.4844(16)$ \\
$\mathrm{N} 2-\mathrm{N} 3$ & $1.1321(18)$ & $\mathrm{N} 2^{*}-\mathrm{N} 3^{*}$ & $1.1337(18)$ \\
$\mathrm{C} 1-\mathrm{H} 1$ & 0.9500 & $\mathrm{C} 1^{*}-\mathrm{H} 1^{*}$ & 0.9500 \\
$\mathrm{C} 1-\mathrm{C} 2$ & $1.4028(18)$ & $\mathrm{C} 1^{*}-\mathrm{C} 2^{*}$ & $1.4029(17)$ \\
$\mathrm{C} 1-\mathrm{C} 9 \mathrm{~A}$ & $1.3833(18)$ & $\mathrm{C} 1^{*}-\mathrm{C} 9 \mathrm{~A}^{*}$ & $1.3837(17)$ \\
$\mathrm{C} 2-\mathrm{C} 3$ & $1.4200(17)$ & $\mathrm{C} 2^{*}-\mathrm{C} 3^{*}$ & $1.4188(17)$ \\
$\mathrm{C} 2-\mathrm{C} 12$ & $1.4766(18)$ & $\mathrm{C} 2^{*}-\mathrm{C} 12^{*}$ & $1.4716(17)$ \\
$\mathrm{C} 3-\mathrm{C} 4$ & $1.3922(17)$ & $\mathrm{C} 3^{*}-\mathrm{C} 4^{*}$ & $1.3905(17)$ \\
$\mathrm{C} 3-\mathrm{C} 13$ & $1.5064(18)$ & $\mathrm{C} 3^{*}-\mathrm{C} 13^{*}$ & $1.5180(17)$ \\
$\mathrm{C} 4-\mathrm{H} 4$ & 0.9500 & $\mathrm{C} 4^{*}-\mathrm{H} 4^{*}$ & 0.9500 \\
$\mathrm{C} 4-\mathrm{C} 4 \mathrm{~A}$ & $1.3907(17)$ & $\mathrm{C} 4^{*}-\mathrm{C} 4 \mathrm{~A}^{*}$ & $1.3981(17)$ \\
$\mathrm{C} 4 \mathrm{~A}-\mathrm{C} 4 \mathrm{~B}$ & $1.4632(17)$ & $\mathrm{C} 4 \mathrm{~A}^{*}-\mathrm{C} 4 \mathrm{~B}^{*}$ & $1.4641(17)$
\end{tabular}




\begin{tabular}{|c|c|c|c|}
\hline C4A-C9A & $1.4050(17)$ & $\mathrm{C} 4 \mathrm{~A} *-\mathrm{C} 9 \mathrm{~A}^{*}$ & $1.4007(17)$ \\
\hline $\mathrm{C} 4 \mathrm{~B}-\mathrm{C} 5$ & $1.3928(17)$ & $\mathrm{C} 4 \mathrm{~B}^{*}-\mathrm{C} 5^{*}$ & $1.3925(17)$ \\
\hline $\mathrm{C} 4 \mathrm{~B}-\mathrm{C} 8 \mathrm{~A}$ & $1.4027(17)$ & $\mathrm{C} 4 \mathrm{~B}^{*}-\mathrm{C} 8 \mathrm{~A}^{*}$ & $1.4053(17)$ \\
\hline C5-H5 & 0.9500 & $\mathrm{C} 5^{*}-\mathrm{H} 5^{*}$ & 0.9500 \\
\hline C5-C6 & $1.3910(18)$ & $\mathrm{C} 5^{*}-\mathrm{C} 6^{*}$ & $1.3926(17)$ \\
\hline C6-H6 & 0.9500 & $\mathrm{C} 6^{*}-\mathrm{H} 6^{*}$ & 0.9500 \\
\hline $\mathrm{C} 6-\mathrm{C} 7$ & $1.3943(18)$ & $\mathrm{C} 6 *-\mathrm{C} 7 *$ & $1.3922(18)$ \\
\hline $\mathrm{C} 7-\mathrm{C} 8$ & $1.3933(18)$ & $\mathrm{C} 7 *-\mathrm{C} 8 *$ & $1.3944(18)$ \\
\hline C8-H8 & 0.9500 & $\mathrm{C} 8 *-\mathrm{H} 8 *$ & 0.9500 \\
\hline $\mathrm{C} 8-\mathrm{C} 8 \mathrm{~A}$ & $1.3892(18)$ & $\mathrm{C} 8 *-\mathrm{C} 8 \mathrm{~A}^{*}$ & $1.3875(17)$ \\
\hline $\mathrm{C} 8 \mathrm{~A}-\mathrm{C} 9$ & $1.5263(17)$ & $\mathrm{C} 8 \mathrm{~A}^{*}-\mathrm{C} 9 *$ & $1.5199(17)$ \\
\hline C9-C9A & $1.5267(18)$ & C9*-C9A* & $1.5219(17)$ \\
\hline C9-C10 & $1.5374(19)$ & $\mathrm{C} 9 *-\mathrm{C} 10 *$ & $1.5353(17)$ \\
\hline C9-C11 & $1.5409(19)$ & $\mathrm{C} 9 *-\mathrm{C} 11 *$ & $1.5335(17)$ \\
\hline C10-H10A & 0.9800 & C10*-H10D & 0.9800 \\
\hline C10-H10B & 0.9800 & C10*-H10E & 0.9800 \\
\hline $\mathrm{C} 10-\mathrm{H} 10 \mathrm{C}$ & 0.9800 & $\mathrm{C} 10^{*}-\mathrm{H} 10 \mathrm{~F}$ & 0.9800 \\
\hline C11-H11A & 0.9800 & $\mathrm{C} 11^{*}-\mathrm{H} 11 \mathrm{D}$ & 0.9800 \\
\hline C11-H11B & 0.9800 & $\mathrm{C} 11^{*}-\mathrm{H} 11 \mathrm{E}$ & 0.9800 \\
\hline $\mathrm{C} 11-\mathrm{H} 11 \mathrm{C}$ & 0.9800 & $\mathrm{C} 11^{*}-\mathrm{H} 11 \mathrm{~F}$ & 0.9800 \\
\hline $\mathrm{C} 12-\mathrm{H} 12$ & 0.9500 & $\mathrm{C} 12^{*}-\mathrm{H} 12^{*}$ & 0.9500 \\
\hline C13-H13A & 0.9900 & $\mathrm{C} 13^{*}-\mathrm{H} 13 \mathrm{C}$ & 0.9900 \\
\hline C13-H13B & 0.9900 & $\mathrm{C} 13 *-\mathrm{H} 13 \mathrm{D}$ & 0.9900 \\
\hline
\end{tabular}

Table S5: Bond Angles for 5.

\begin{tabular}{|c|c|c|c|}
\hline Atom-Atom-Atom & Angle $\left[{ }^{\circ}\right]$ & Atom-Atom-Atom & Angle $\left[{ }^{\circ}\right]$ \\
\hline N2-N1-C13 & $114.01(11)$ & $\mathrm{N} 2 *-\mathrm{N} 1 *-\mathrm{C} 13 *$ & $115.47(11)$ \\
\hline N3-N2-N1 & $173.21(14)$ & $\mathrm{N} 3 *-\mathrm{N} 2 *-\mathrm{N} 1 *$ & $172.57(14)$ \\
\hline $\mathrm{C} 2-\mathrm{C} 1-\mathrm{H} 1$ & 120.0 & $\mathrm{C} 2 *-\mathrm{C} 1 *-\mathrm{H} 1 *$ & 120.1 \\
\hline $\mathrm{C} 9 \mathrm{~A}-\mathrm{C} 1-\mathrm{H} 1$ & 120.0 & $\mathrm{C} 9 \mathrm{~A}^{*}-\mathrm{C} 1 *-\mathrm{H} 1 *$ & 120.1 \\
\hline $\mathrm{C} 9 \mathrm{~A}-\mathrm{C} 1-\mathrm{C} 2$ & $119.98(12)$ & $\mathrm{C} 9 \mathrm{~A}^{*}-\mathrm{C} 1 *-\mathrm{C} 2 *$ & $119.76(11)$ \\
\hline $\mathrm{C} 1-\mathrm{C} 2-\mathrm{C} 3$ & $120.73(11)$ & $\mathrm{C} 1 *-\mathrm{C} 2 *-\mathrm{C} 3 *$ & $120.79(11)$ \\
\hline $\mathrm{C} 1-\mathrm{C} 2-\mathrm{C} 12$ & $116.65(11)$ & $\mathrm{C} 1 *-\mathrm{C} 2 *-\mathrm{C} 12 *$ & $115.95(11)$ \\
\hline $\mathrm{C} 3-\mathrm{C} 2-\mathrm{C} 12$ & $122.62(12)$ & $\mathrm{C} 3 *-\mathrm{C} 2 *-\mathrm{C} 12 *$ & $123.25(11)$ \\
\hline $\mathrm{C} 2-\mathrm{C} 3-\mathrm{C} 13$ & $120.59(11)$ & $\mathrm{C} 2 *-\mathrm{C} 3 *-\mathrm{C} 13 *$ & $120.55(11)$ \\
\hline $\mathrm{C} 4-\mathrm{C} 3-\mathrm{C} 2$ & $118.72(11)$ & $\mathrm{C} 4 *-\mathrm{C} 3 *-\mathrm{C} 2 *$ & $118.76(11)$ \\
\hline $\mathrm{C} 4-\mathrm{C} 3-\mathrm{C} 13$ & $120.69(11)$ & $\mathrm{C} 4 *-\mathrm{C} 3 *-\mathrm{C} 13^{*}$ & $120.68(11)$ \\
\hline
\end{tabular}




\begin{tabular}{|c|c|}
\hline $\mathrm{C} 3-\mathrm{C} 4-\mathrm{H} 4$ & 120.1 \\
\hline $\mathrm{C} 4 \mathrm{~A}-\mathrm{C} 4-\mathrm{C} 3$ & $119.89(11)$ \\
\hline $\mathrm{C} 4 \mathrm{~A}-\mathrm{C} 4-\mathrm{H} 4$ & 120.1 \\
\hline $\mathrm{C} 4-\mathrm{C} 4 \mathrm{~A}-\mathrm{C} 4 \mathrm{~B}$ & $129.90(11)$ \\
\hline $\mathrm{C} 4-\mathrm{C} 4 \mathrm{~A}-\mathrm{C} 9 \mathrm{~A}$ & $121.56(11)$ \\
\hline C9A-C4A-C4B & $108.52(11)$ \\
\hline $\mathrm{C} 5-\mathrm{C} 4 \mathrm{~B}-\mathrm{C} 4 \mathrm{~A}$ & $130.36(12)$ \\
\hline $\mathrm{C} 5-\mathrm{C} 4 \mathrm{~B}-\mathrm{C} 8 \mathrm{~A}$ & $121.10(12)$ \\
\hline $\mathrm{C} 8 \mathrm{~A}-\mathrm{C} 4 \mathrm{~B}-\mathrm{C} 4 \mathrm{~A}$ & $108.53(11)$ \\
\hline $\mathrm{C} 4 \mathrm{~B}-\mathrm{C} 5-\mathrm{H} 5$ & 120.5 \\
\hline C6-C5-C4B & $118.92(12)$ \\
\hline C6-C5-H5 & 120.5 \\
\hline C5-C6-H6 & 120.4 \\
\hline $\mathrm{C} 5-\mathrm{C} 6-\mathrm{C} 7$ & $119.15(12)$ \\
\hline C7-C6-H6 & 120.4 \\
\hline $\mathrm{C} 6-\mathrm{C} 7-\mathrm{Br} 1$ & 117.83(9) \\
\hline $\mathrm{C} 8-\mathrm{C} 7-\mathrm{Br} 1$ & $119.29(10)$ \\
\hline $\mathrm{C} 8-\mathrm{C} 7-\mathrm{C} 6$ & $122.88(12)$ \\
\hline C7-C8-H8 & 121.3 \\
\hline $\mathrm{C} 8 \mathrm{~A}-\mathrm{C} 8-\mathrm{C} 7$ & $117.40(12)$ \\
\hline $\mathrm{C} 8 \mathrm{~A}-\mathrm{C} 8-\mathrm{H} 8$ & 121.3 \\
\hline $\mathrm{C} 4 \mathrm{~B}-\mathrm{C} 8 \mathrm{~A}-\mathrm{C} 9$ & $111.03(11)$ \\
\hline $\mathrm{C} 8-\mathrm{C} 8 \mathrm{~A}-\mathrm{C} 4 \mathrm{~B}$ & $120.56(12)$ \\
\hline C8-C8A-C9 & $128.41(12)$ \\
\hline $\mathrm{C} 8 \mathrm{~A}-\mathrm{C} 9-\mathrm{C} 9 \mathrm{~A}$ & $100.99(10)$ \\
\hline C8A-C9-C10 & $111.47(11)$ \\
\hline C8A-C9-C11 & $110.98(11)$ \\
\hline C9A-C9-C10 & $112.16(11)$ \\
\hline C9A-C9-C11 & $110.84(11)$ \\
\hline C10-C9-C11 & $110.14(11)$ \\
\hline $\mathrm{C} 1-\mathrm{C} 9 \mathrm{~A}-\mathrm{C} 4 \mathrm{~A}$ & $119.11(12)$ \\
\hline $\mathrm{C} 1-\mathrm{C} 9 \mathrm{~A}-\mathrm{C} 9$ & $129.96(11)$ \\
\hline $\mathrm{C} 4 \mathrm{~A}-\mathrm{C} 9 \mathrm{~A}-\mathrm{C} 9$ & $110.91(11)$ \\
\hline C9-C10-H10A & 109.5 \\
\hline C9-C10-H10B & 109.5 \\
\hline C9-C10-H10C & 109.5 \\
\hline $\mathrm{H} 10 \mathrm{~A}-\mathrm{C} 10-\mathrm{H} 10 \mathrm{~B}$ & 109.5 \\
\hline
\end{tabular}

\begin{tabular}{|c|c|}
\hline $\mathrm{C} 3 *-\mathrm{C} 4 *-\mathrm{H} 4 *$ & 120.0 \\
\hline $\mathrm{C} 3 *-\mathrm{C} 4 *-\mathrm{C} 4 \mathrm{~A}^{*}$ & $119.97(11)$ \\
\hline $\mathrm{C} 4 \mathrm{~A}^{*}-\mathrm{C} 4 *-\mathrm{H} 4 *$ & 120.0 \\
\hline $\mathrm{C} 4 *-\mathrm{C} 4 \mathrm{~A}^{*}-\mathrm{C} 4 \mathrm{~B} *$ & $130.38(11)$ \\
\hline $\mathrm{C} 4 *-\mathrm{C} 4 \mathrm{~A}^{*}-\mathrm{C} 9 \mathrm{~A}^{*}$ & $121.10(11)$ \\
\hline $\mathrm{C} 9 \mathrm{~A}^{*}-\mathrm{C} 4 \mathrm{~A}^{*}-\mathrm{C} 4 \mathrm{~B} *$ & $108.49(10)$ \\
\hline $\mathrm{C} 5^{*}-\mathrm{C} 4 \mathrm{~B}^{*}-\mathrm{C} 4 \mathrm{~A}^{*}$ & $131.05(11)$ \\
\hline $\mathrm{C} 5^{*}-\mathrm{C} 4 \mathrm{~B}^{*}-\mathrm{C} 8 \mathrm{~A}^{*}$ & $120.85(11)$ \\
\hline $\mathrm{C} 8 \mathrm{~A}^{*}-\mathrm{C} 4 \mathrm{~B}^{*}-\mathrm{C} 4 \mathrm{~A}^{*}$ & $108.09(10)$ \\
\hline $\mathrm{C} 4 \mathrm{~B}^{*}-\mathrm{C} 5^{*}-\mathrm{H} 5^{*}$ & 120.5 \\
\hline $\mathrm{C} 4 \mathrm{~B}^{*}-\mathrm{C} 5^{*}-\mathrm{C} 6 *$ & $118.91(11)$ \\
\hline $\mathrm{C} 6 *-\mathrm{C} 5^{*}-\mathrm{H} 5^{*}$ & 120.5 \\
\hline $\mathrm{C} 5^{*}-\mathrm{C} 6^{*}-\mathrm{H} 6^{*}$ & 120.3 \\
\hline $\mathrm{C} 7 *-\mathrm{C} 6 *-\mathrm{C} 5 *$ & $119.37(11)$ \\
\hline $\mathrm{C} 7 *-\mathrm{C} 6 *-\mathrm{H} 6^{*}$ & 120.3 \\
\hline $\mathrm{C} 6 *-\mathrm{C} 7 *-\mathrm{Br} 1 *$ & $118.55(9)$ \\
\hline $\mathrm{C} 6 *-\mathrm{C} 7 *-\mathrm{C} 8 *$ & $122.69(11)$ \\
\hline $\mathrm{C} 8 *-\mathrm{C} 7 *-\mathrm{Br} 1 *$ & $118.77(9)$ \\
\hline $\mathrm{C} 7 *-\mathrm{C} 8 *-\mathrm{H} 8 *$ & 121.3 \\
\hline $\mathrm{C} 8 \mathrm{~A}^{*}-\mathrm{C} 8 *-\mathrm{C} 7 *$ & $117.44(11)$ \\
\hline $\mathrm{C} 8 \mathrm{~A}^{*}-\mathrm{C} 8 *-\mathrm{H} 8 *$ & 121.3 \\
\hline $\mathrm{C} 4 \mathrm{~B} *-\mathrm{C} 8 \mathrm{~A}^{*}-\mathrm{C} 9 *$ & $111.33(10)$ \\
\hline $\mathrm{C} 8 *-\mathrm{C} 8 \mathrm{~A}^{*}-\mathrm{C} 4 \mathrm{~B} *$ & $120.74(11)$ \\
\hline $\mathrm{C} 8 *-\mathrm{C} 8 \mathrm{~A}^{*}-\mathrm{C} 9 *$ & $127.93(11)$ \\
\hline $\mathrm{C} 8 \mathrm{~A}^{*}-\mathrm{C} 9 *-\mathrm{C} 9 \mathrm{~A}^{*}$ & $100.86(9)$ \\
\hline $\mathrm{C} 8 \mathrm{~A}^{*}-\mathrm{C} 9 *-\mathrm{C} 10^{*}$ & $111.25(10)$ \\
\hline $\mathrm{C} 8 \mathrm{~A} *-\mathrm{C} 9 *-\mathrm{C} 11 *$ & $111.67(10)$ \\
\hline $\mathrm{C} 9 \mathrm{~A}^{*}-\mathrm{C} 9 *-\mathrm{C} 10^{*}$ & $111.31(10)$ \\
\hline $\mathrm{C} 9 \mathrm{~A}^{*}-\mathrm{C} 9 *-\mathrm{C} 11^{*}$ & $111.24(10)$ \\
\hline $\mathrm{C} 11 *-\mathrm{C} 9 *-\mathrm{C} 10 *$ & $110.23(10)$ \\
\hline $\mathrm{C} 1 *-\mathrm{C} 9 \mathrm{~A}^{*}-\mathrm{C} 4 \mathrm{~A} *$ & $119.58(11)$ \\
\hline $\mathrm{C} 1 *$-C9A*-C $9 *$ & $129.19(11)$ \\
\hline $\mathrm{C} 4 \mathrm{~A}^{*}-\mathrm{C} 9 \mathrm{~A} *-\mathrm{C} 9 *$ & $111.22(10)$ \\
\hline $\mathrm{C} 9 *-\mathrm{C} 10^{*}-\mathrm{H} 10 \mathrm{D}$ & 109.5 \\
\hline $\mathrm{C} 9 *-\mathrm{C} 10 *-\mathrm{H} 10 \mathrm{E}$ & 109.5 \\
\hline $\mathrm{C} 9 *-\mathrm{C} 10 *-\mathrm{H} 10 \mathrm{~F}$ & 109.5 \\
\hline $\mathrm{H} 10 \mathrm{D}-\mathrm{C} 10^{*}-\mathrm{H} 10 \mathrm{E}$ & 109.5 \\
\hline
\end{tabular}




\begin{tabular}{|c|c|c|c|}
\hline $\mathrm{H} 10 \mathrm{~A}-\mathrm{C} 10-\mathrm{H} 10 \mathrm{C}$ & 109.5 & $\mathrm{H} 10 \mathrm{D}-\mathrm{C} 10^{*}-\mathrm{H} 10 \mathrm{~F}$ & 109.5 \\
\hline H10B-C10-H10C & 109.5 & $\mathrm{H} 10 \mathrm{E}-\mathrm{C} 10^{*}-\mathrm{H} 10 \mathrm{~F}$ & 109.5 \\
\hline C9-C11-H11A & 109.5 & $\mathrm{C} 9 *-\mathrm{C} 11^{*}-\mathrm{H} 11 \mathrm{D}$ & 109.5 \\
\hline C9-C11-H11B & 109.5 & $\mathrm{C} 9 *-\mathrm{C} 11 *-\mathrm{H} 11 \mathrm{E}$ & 109.5 \\
\hline C9-C11-H11C & 109.5 & $\mathrm{C} 9 *-\mathrm{C} 11 *-\mathrm{H} 11 \mathrm{~F}$ & 109.5 \\
\hline $\mathrm{H} 11 \mathrm{~A}-\mathrm{C} 11-\mathrm{H} 11 \mathrm{~B}$ & 109.5 & $\mathrm{H} 11 \mathrm{D}-\mathrm{C} 11^{*}-\mathrm{H} 11 \mathrm{E}$ & 109.5 \\
\hline $\mathrm{H} 11 \mathrm{~A}-\mathrm{C} 11-\mathrm{H} 11 \mathrm{C}$ & 109.5 & $\mathrm{H} 11 \mathrm{D}-\mathrm{C} 11^{*}-\mathrm{H} 11 \mathrm{~F}$ & 109.5 \\
\hline $\mathrm{H} 11 \mathrm{~B}-\mathrm{C} 11-\mathrm{H} 11 \mathrm{C}$ & 109.5 & $\mathrm{H} 11 \mathrm{E}-\mathrm{C} 11{ }^{*}-\mathrm{H} 11 \mathrm{~F}$ & 109.5 \\
\hline $\mathrm{O} 1-\mathrm{C} 12-\mathrm{C} 2$ & $125.83(12)$ & $\mathrm{O} 1 *-\mathrm{C} 12 *-\mathrm{C} 2 *$ & $125.87(12)$ \\
\hline $\mathrm{O} 1-\mathrm{C} 12-\mathrm{H} 12$ & 117.1 & $\mathrm{O} 1 *-\mathrm{C} 12 *-\mathrm{H} 12 *$ & 117.1 \\
\hline $\mathrm{C} 2-\mathrm{C} 12-\mathrm{H} 12$ & 117.1 & $\mathrm{C} 2 *-\mathrm{C} 12 *-\mathrm{H} 12 *$ & 117.1 \\
\hline N1-C13-C3 & $108.94(10)$ & $\mathrm{N} 1 *-\mathrm{C} 13 *-\mathrm{C} 3 *$ & $112.68(10)$ \\
\hline N1-C13-H13A & 109.9 & $\mathrm{~N} 1{ }^{*}-\mathrm{C} 13 *-\mathrm{H} 13 \mathrm{C}$ & 109.1 \\
\hline N1-C13-H13B & 109.9 & $\mathrm{~N} 1{ }^{*}-\mathrm{C} 13{ }^{*}-\mathrm{H} 13 \mathrm{D}$ & 109.1 \\
\hline $\mathrm{C} 3-\mathrm{C} 13-\mathrm{H} 13 \mathrm{~A}$ & 109.9 & $\mathrm{C} 3{ }^{*}-\mathrm{C} 13^{*}-\mathrm{H} 13 \mathrm{C}$ & 109.1 \\
\hline C3-C13-H13B & 109.9 & $\mathrm{C} 3 *-\mathrm{C} 13^{*}-\mathrm{H} 13 \mathrm{D}$ & 109.1 \\
\hline $\mathrm{H} 13 \mathrm{~A}-\mathrm{C} 13-\mathrm{H} 13 \mathrm{~B}$ & 108.3 & $\mathrm{H} 13 \mathrm{C}-\mathrm{C} 13^{*}-\mathrm{H} 13 \mathrm{D}$ & 107.8 \\
\hline
\end{tabular}

Table S5: Torsion Angles for 5.

\begin{tabular}{|c|c|c|c|}
\hline Atom-Atom-Atom-Atom & Torsion Angle $\left[{ }^{\circ}\right]$ & Atom-Atom-Atom-Atom & Torsion Angle [ $\left.{ }^{\circ}\right]$ \\
\hline $\mathrm{Br} 1-\mathrm{C} 7-\mathrm{C} 8-\mathrm{C} 8 \mathrm{~A}$ & $-179.52(9)$ & $\mathrm{Br}^{*} * \mathrm{C} 7 *-\mathrm{C} 8 *-\mathrm{C} 8 \mathrm{~A}^{*}$ & 178.96(9) \\
\hline $\mathrm{N} 2-\mathrm{N} 1-\mathrm{C} 13-\mathrm{C} 3$ & $174.96(12)$ & $\mathrm{N} 2{ }^{*}-\mathrm{N} 1 *-\mathrm{C} 13^{*}-\mathrm{C} 3 *$ & $89.13(14)$ \\
\hline $\mathrm{C} 1-\mathrm{C} 2-\mathrm{C} 3-\mathrm{C} 4$ & $1.34(19)$ & $\mathrm{C} 1 *-\mathrm{C} 2 *-\mathrm{C} 3 *-\mathrm{C} 4 *$ & $1.11(18)$ \\
\hline $\mathrm{C} 1-\mathrm{C} 2-\mathrm{C} 3-\mathrm{C} 13$ & $-178.51(12)$ & $\mathrm{C} 1 *-\mathrm{C} 2 *-\mathrm{C} 3 *-\mathrm{C} 13 *$ & $-177.50(11)$ \\
\hline $\mathrm{C} 1-\mathrm{C} 2-\mathrm{C} 12-\mathrm{O} 1$ & $-176.60(14)$ & $\mathrm{C} 1 *-\mathrm{C} 2 *-\mathrm{C} 12 *-\mathrm{O} 1 *$ & $-166.75(13)$ \\
\hline $\mathrm{C} 2-\mathrm{C} 1-\mathrm{C} 9 \mathrm{~A}-\mathrm{C} 4 \mathrm{~A}$ & $-0.49(19)$ & $\mathrm{C} 2 *-\mathrm{C} 1 *-\mathrm{C} 9 \mathrm{~A} *-\mathrm{C} 4 \mathrm{~A}^{*}$ & $1.37(18)$ \\
\hline $\mathrm{C} 2-\mathrm{C} 1-\mathrm{C} 9 \mathrm{~A}-\mathrm{C} 9$ & 177.71(13) & $\mathrm{C} 2 *-\mathrm{C} 1 *-\mathrm{C} 9 \mathrm{~A}^{*}-\mathrm{C} 9 *$ & $-179.65(12)$ \\
\hline $\mathrm{C} 2-\mathrm{C} 3-\mathrm{C} 4-\mathrm{C} 4 \mathrm{~A}$ & $-0.87(18)$ & $\mathrm{C} 2 *-\mathrm{C} 3 *-\mathrm{C} 4 *-\mathrm{C} 4 \mathrm{~A}^{*}$ & $0.36(18)$ \\
\hline $\mathrm{C} 2-\mathrm{C} 3-\mathrm{C} 13-\mathrm{N} 1$ & $171.26(11)$ & $\mathrm{C} 2{ }^{*}-\mathrm{C} 3{ }^{*}-\mathrm{C} 13^{*}-\mathrm{N} 1 *$ & $157.56(11)$ \\
\hline $\mathrm{C} 3-\mathrm{C} 2-\mathrm{C} 12-\mathrm{O} 1$ & $2.9(2)$ & $\mathrm{C} 3 *-\mathrm{C} 2 *-\mathrm{C} 12 *-\mathrm{O} 1 *$ & $12.4(2)$ \\
\hline $\mathrm{C} 3-\mathrm{C} 4-\mathrm{C} 4 \mathrm{~A}-\mathrm{C} 4 \mathrm{~B}$ & $-178.81(12)$ & $\mathrm{C} 3 *-\mathrm{C} 4 *-\mathrm{C} 4 \mathrm{~A}^{*}-\mathrm{C} 4 \mathrm{~B} *$ & $-178.88(12)$ \\
\hline $\mathrm{C} 3-\mathrm{C} 4-\mathrm{C} 4 \mathrm{~A}-\mathrm{C} 9 \mathrm{~A}$ & $-0.27(19)$ & $\mathrm{C} 3 *-\mathrm{C} 4 *-\mathrm{C} 4 \mathrm{~A}^{*}-\mathrm{C} 9 \mathrm{~A}^{*}$ & $-0.96(18)$ \\
\hline $\mathrm{C} 4-\mathrm{C} 3-\mathrm{C} 13-\mathrm{N} 1$ & $-8.58(16)$ & $\mathrm{C} 4 *-\mathrm{C} 3 *-\mathrm{C} 13 *-\mathrm{N} 1 *$ & $-21.03(16)$ \\
\hline $\mathrm{C} 4-\mathrm{C} 4 \mathrm{~A}-\mathrm{C} 4 \mathrm{~B}-\mathrm{C} 5$ & $-1.5(2)$ & $\mathrm{C} 4 *-\mathrm{C} 4 \mathrm{~A}^{*}-\mathrm{C} 4 \mathrm{~B}^{*}-\mathrm{C} 5^{*}$ & $-0.1(2)$ \\
\hline $\mathrm{C} 4-\mathrm{C} 4 \mathrm{~A}-\mathrm{C} 4 \mathrm{~B}-\mathrm{C} 8 \mathrm{~A}$ & $177.87(13)$ & $\mathrm{C} 4 *-\mathrm{C} 4 \mathrm{~A}^{*}-\mathrm{C} 4 \mathrm{~B}^{*}-\mathrm{C} 8 \mathrm{~A}^{*}$ & $178.41(13)$ \\
\hline $\mathrm{C} 4-\mathrm{C} 4 \mathrm{~A}-\mathrm{C} 9 \mathrm{~A}-\mathrm{C} 1$ & $0.96(19)$ & $\mathrm{C} 4 *-\mathrm{C} 4 \mathrm{~A}^{*}-\mathrm{C} 9 \mathrm{~A}^{*}-\mathrm{C} 1^{*}$ & $0.09(18)$ \\
\hline $\mathrm{C} 4-\mathrm{C} 4 \mathrm{~A}-\mathrm{C} 9 \mathrm{~A}-\mathrm{C} 9$ & $-177.56(11)$ & $\mathrm{C} 4 *-\mathrm{C} 4 \mathrm{~A}^{*}-\mathrm{C} 9 \mathrm{~A}^{*}-\mathrm{C} 9 *$ & $-179.06(11)$ \\
\hline
\end{tabular}




\begin{tabular}{|c|c|c|c|}
\hline $\mathrm{C} 4 \mathrm{~A}-\mathrm{C} 4 \mathrm{~B}-\mathrm{C} 5-\mathrm{C} 6$ & $179.44(13)$ & $\mathrm{C} 4 \mathrm{~A}^{*}-\mathrm{C} 4 \mathrm{~B}^{*}-\mathrm{C} 5^{*}-\mathrm{C} 6^{*}$ & $178.13(12)$ \\
\hline $\mathrm{C} 4 \mathrm{~A}-\mathrm{C} 4 \mathrm{~B}-\mathrm{C} 8 \mathrm{~A}-\mathrm{C} 8$ & $-179.69(11)$ & $\mathrm{C} 4 \mathrm{~A}^{*-}-\mathrm{C} 4 \mathrm{~B}^{*}-\mathrm{C} 8 \mathrm{~A}^{*}-\mathrm{C} 8 *$ & $-178.84(11)$ \\
\hline $\mathrm{C} 4 \mathrm{~A}-\mathrm{C} 4 \mathrm{~B}-\mathrm{C} 8 \mathrm{~A}-\mathrm{C} 9$ & $0.06(14)$ & $\mathrm{C} 4 \mathrm{~A}^{*}-\mathrm{C} 4 \mathrm{~B}^{*}-\mathrm{C} 8 \mathrm{~A}^{*}-\mathrm{C} 9 *$ & $0.27(14)$ \\
\hline $\mathrm{C} 4 \mathrm{~B}-\mathrm{C} 4 \mathrm{~A}-\mathrm{C} 9 \mathrm{~A}-\mathrm{C} 1$ & $179.78(12)$ & $\mathrm{C} 4 \mathrm{~B}^{*}-\mathrm{C} 4 \mathrm{~A}^{*}-\mathrm{C} 9 \mathrm{~A}^{*}-\mathrm{C} 1^{*}$ & $178.42(11)$ \\
\hline $\mathrm{C} 4 \mathrm{~B}-\mathrm{C} 4 \mathrm{~A}-\mathrm{C} 9 \mathrm{~A}-\mathrm{C} 9$ & $1.26(14)$ & $\mathrm{C} 4 \mathrm{~B}^{*}-\mathrm{C} 4 \mathrm{~A}^{*}-\mathrm{C} 9 \mathrm{~A}^{*}-\mathrm{C} 9 *$ & $-0.73(14)$ \\
\hline $\mathrm{C} 4 \mathrm{~B}-\mathrm{C} 5-\mathrm{C} 6-\mathrm{C} 7$ & $0.05(19)$ & $\mathrm{C} 4 \mathrm{~B}^{*}-\mathrm{C} 5^{*}-\mathrm{C} 6^{*}-\mathrm{C} 7 *$ & $0.23(19)$ \\
\hline C4B-C8A-C9-C9A & $0.64(14)$ & $\mathrm{C} 4 \mathrm{~B}^{*}-\mathrm{C} 8 \mathrm{~A}^{*}-\mathrm{C} 9^{*}-\mathrm{C} 9 \mathrm{~A}^{*}$ & $-0.65(13)$ \\
\hline $\mathrm{C} 4 \mathrm{~B}-\mathrm{C} 8 \mathrm{~A}-\mathrm{C} 9-\mathrm{C} 10$ & $119.93(12)$ & $\mathrm{C} 4 \mathrm{~B}^{*}-\mathrm{C} 8 \mathrm{~A}^{*}-\mathrm{C} 9 *-\mathrm{C} 10^{*}$ & $117.48(11)$ \\
\hline $\mathrm{C} 4 \mathrm{~B}-\mathrm{C} 8 \mathrm{~A}-\mathrm{C} 9-\mathrm{C} 11$ & $-116.91(12)$ & $\mathrm{C} 4 \mathrm{~B}^{*}-\mathrm{C} 8 \mathrm{~A}^{*}-\mathrm{C} 9 *-\mathrm{C} 11^{*}$ & $-118.90(12)$ \\
\hline $\mathrm{C} 5-\mathrm{C} 4 \mathrm{~B}-\mathrm{C} 8 \mathrm{~A}-\mathrm{C} 8$ & $-0.21(19)$ & $\mathrm{C} 5^{*}-\mathrm{C} 4 \mathrm{~B}^{*}-\mathrm{C} 8 \mathrm{~A}^{*}-\mathrm{C} 8^{*}$ & $-0.14(19)$ \\
\hline C5-C4B-C8A-C9 & $179.54(11)$ & $\mathrm{C} 5^{*}-\mathrm{C} 4 \mathrm{~B}^{*}-\mathrm{C} 8 \mathrm{~A}^{*}-\mathrm{C} 9^{*}$ & $178.97(11)$ \\
\hline C5-C6-C7-Br1 & $179.42(10)$ & $\mathrm{C} 5 *-\mathrm{C} 6 *-\mathrm{C} 7 *-\mathrm{Br} 1 *$ & $-179.32(9)$ \\
\hline $\mathrm{C} 5-\mathrm{C} 6-\mathrm{C} 7-\mathrm{C} 8$ & $-0.1(2)$ & $\mathrm{C} 5 *-\mathrm{C} 6 *-\mathrm{C} 7 *-\mathrm{C} 8 *$ & $0.13(19)$ \\
\hline $\mathrm{C} 6-\mathrm{C} 7-\mathrm{C} 8-\mathrm{C} 8 \mathrm{~A}$ & $-0.03(19)$ & $\mathrm{C} 6 *-\mathrm{C} 7 *-\mathrm{C} 8 *-\mathrm{C} 8 \mathrm{~A}^{*}$ & $-0.49(19)$ \\
\hline $\mathrm{C} 7-\mathrm{C} 8-\mathrm{C} 8 \mathrm{~A}-\mathrm{C} 4 \mathrm{~B}$ & $0.17(19)$ & $\mathrm{C} 7 *-\mathrm{C} 8 *-\mathrm{C} 8 \mathrm{~A}^{*}-\mathrm{C} 4 \mathrm{~B} *$ & $0.49(18)$ \\
\hline $\mathrm{C} 7-\mathrm{C} 8-\mathrm{C} 8 \mathrm{~A}-\mathrm{C} 9$ & $-179.53(12)$ & $\mathrm{C} 7 *-\mathrm{C} 8 *-\mathrm{C} 8 \mathrm{~A}^{*}-\mathrm{C} 9 *$ & $-178.46(12)$ \\
\hline C8-C8A-C9-C9A & $-179.64(13)$ & $\mathrm{C} 8 *-\mathrm{C} 8 \mathrm{~A}^{*}-\mathrm{C} 9 *-\mathrm{C} 9 \mathrm{~A}^{*}$ & $178.38(12)$ \\
\hline $\mathrm{C} 8-\mathrm{C} 8 \mathrm{~A}-\mathrm{C} 9-\mathrm{C} 10$ & $-60.35(18)$ & $\mathrm{C} 8 *-\mathrm{C} 8 \mathrm{~A}^{*}-\mathrm{C} 9 *-\mathrm{C} 10^{*}$ & $-63.49(16)$ \\
\hline $\mathrm{C} 8-\mathrm{C} 8 \mathrm{~A}-\mathrm{C} 9-\mathrm{C} 11$ & $62.81(17)$ & $\mathrm{C} 8 *-\mathrm{C} 8 \mathrm{~A}^{*}-\mathrm{C} 9 *-\mathrm{C} 11^{*}$ & $60.14(16)$ \\
\hline $\mathrm{C} 8 \mathrm{~A}-\mathrm{C} 4 \mathrm{~B}-\mathrm{C} 5-\mathrm{C} 6$ & $0.09(19)$ & $\mathrm{C} 8 \mathrm{~A}^{*}-\mathrm{C} 4 \mathrm{~B}^{*}-\mathrm{C} 5^{*}-\mathrm{C} 6^{*}$ & $-0.23(19)$ \\
\hline $\mathrm{C} 8 \mathrm{~A}-\mathrm{C} 9-\mathrm{C} 9 \mathrm{~A}-\mathrm{C} 1$ & $-179.47(13)$ & $\mathrm{C} 8 \mathrm{~A}^{*}-\mathrm{C} 9 *-\mathrm{C} 9 \mathrm{~A}^{*}-\mathrm{C} 1^{*}$ & $-178.21(12)$ \\
\hline C8A-C9-C9A-C4A & $-1.15(14)$ & $\mathrm{C} 8 \mathrm{~A}^{*-\mathrm{C}} 9 *-\mathrm{C} 9 \mathrm{~A}^{*}-\mathrm{C} 4 \mathrm{~A}^{*}$ & $0.84(13)$ \\
\hline $\mathrm{C} 9 \mathrm{~A}-\mathrm{C} 1-\mathrm{C} 2-\mathrm{C} 3$ & $-0.65(19)$ & $\mathrm{C} 9 \mathrm{~A}^{*}-\mathrm{C} 1 *-\mathrm{C} 2 *-\mathrm{C} 3 *$ & $-1.99(19)$ \\
\hline $\mathrm{C} 9 \mathrm{~A}-\mathrm{C} 1-\mathrm{C} 2-\mathrm{C} 12$ & $178.89(12)$ & $\mathrm{C} 9 \mathrm{~A}^{*}-\mathrm{C} 1 *-\mathrm{C} 2 *-\mathrm{C} 12 *$ & $177.21(11)$ \\
\hline $\mathrm{C} 9 \mathrm{~A}-\mathrm{C} 4 \mathrm{~A}-\mathrm{C} 4 \mathrm{~B}-\mathrm{C} 5$ & $179.76(13)$ & $\mathrm{C} 9 \mathrm{~A}^{*}-\mathrm{C} 4 \mathrm{~A}^{*}-\mathrm{C} 4 \mathrm{~B}^{*}-\mathrm{C} 5^{*}$ & $-178.23(13)$ \\
\hline $\mathrm{C} 9 \mathrm{~A}-\mathrm{C} 4 \mathrm{~A}-\mathrm{C} 4 \mathrm{~B}-\mathrm{C} 8 \mathrm{~A}$ & $-0.82(14)$ & $\mathrm{C} 9 \mathrm{~A}^{*}-\mathrm{C} 4 \mathrm{~A}^{*}-\mathrm{C} 4 \mathrm{~B} *-\mathrm{C} 8 \mathrm{~A} *$ & $0.29(14)$ \\
\hline $\mathrm{C} 10-\mathrm{C} 9-\mathrm{C} 9 \mathrm{~A}-\mathrm{C} 1$ & $61.74(18)$ & $\mathrm{C} 10 *-\mathrm{C} 9 *-\mathrm{C} 9 \mathrm{~A}^{*}-\mathrm{C} 1 *$ & $63.70(16)$ \\
\hline C10-C9-C9A-C4A & $-119.94(12)$ & $\mathrm{C} 10 *-\mathrm{C} 9 *-\mathrm{C} 9 \mathrm{~A} *-\mathrm{C} 4 \mathrm{~A} *$ & $-117.25(11)$ \\
\hline $\mathrm{C} 11-\mathrm{C} 9-\mathrm{C} 9 \mathrm{~A}-\mathrm{C} 1$ & $-61.81(18)$ & $\mathrm{C} 11 *-\mathrm{C} 9 *-\mathrm{C} 9 \mathrm{~A}^{*}-\mathrm{C} 1 *$ & $-59.66(17)$ \\
\hline C11-C9-C9A-C4A & $116.50(12)$ & $\mathrm{C} 11 *-\mathrm{C} 9 *-\mathrm{C} 9 \mathrm{~A} *-\mathrm{C} 4 \mathrm{~A} *$ & $119.39(11)$ \\
\hline $\mathrm{C} 12-\mathrm{C} 2-\mathrm{C} 3-\mathrm{C} 4$ & $-178.18(12)$ & $\mathrm{C} 12^{*}-\mathrm{C} 2 *-\mathrm{C} 3 *-\mathrm{C} 4 *$ & $-178.02(12)$ \\
\hline $\mathrm{C} 12-\mathrm{C} 2-\mathrm{C} 3-\mathrm{C} 13$ & $1.97(19)$ & $\mathrm{C} 12 *-\mathrm{C} 2 *-\mathrm{C} 3 *-\mathrm{C} 13 *$ & $3.37(19)$ \\
\hline $\mathrm{C} 13-\mathrm{C} 3-\mathrm{C} 4-\mathrm{C} 4 \mathrm{~A}$ & $178.97(11)$ & $\mathrm{C} 13 *-\mathrm{C} 3 *-\mathrm{C} 4 *-\mathrm{C} 4 \mathrm{~A} *$ & $178.97(11)$ \\
\hline
\end{tabular}




\subsection{Crystal Packing Views of (5)}

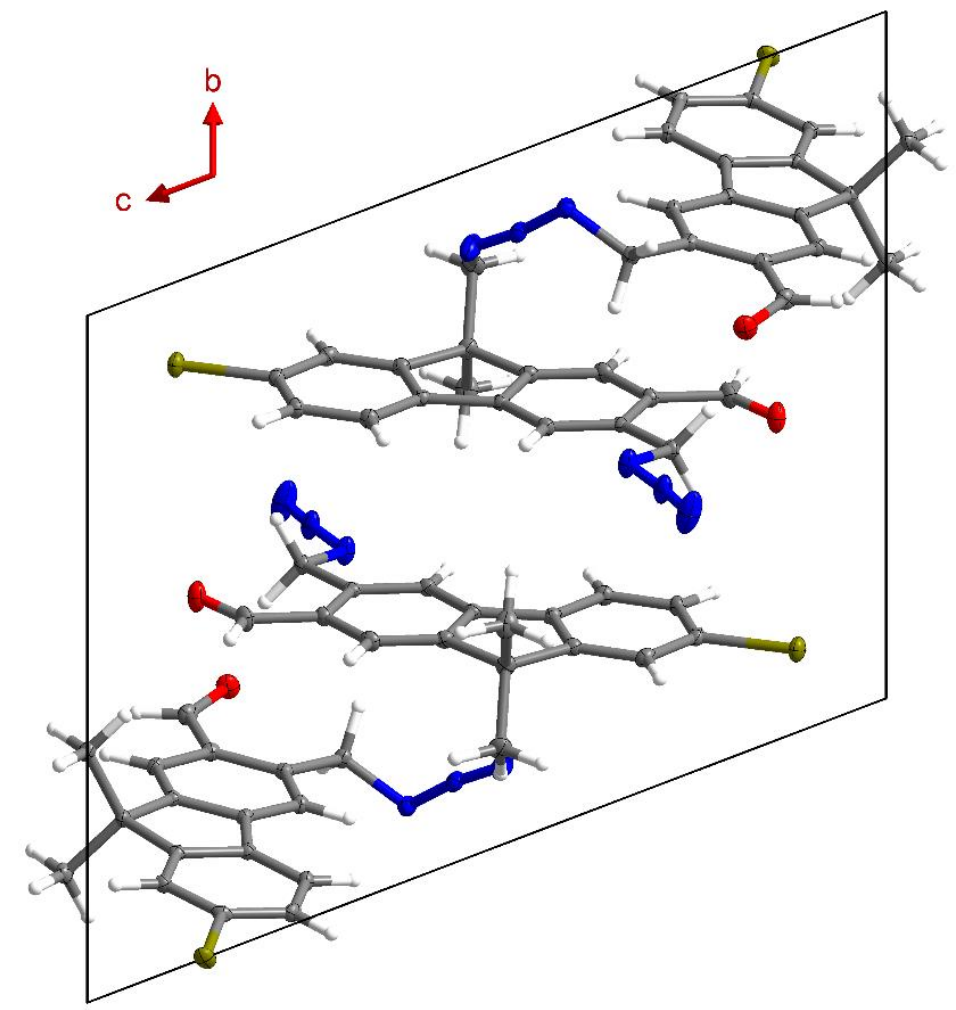

Figure S2: Unit cell packing view of a $\mathbf{5}$ crystal along the $a$-axis.

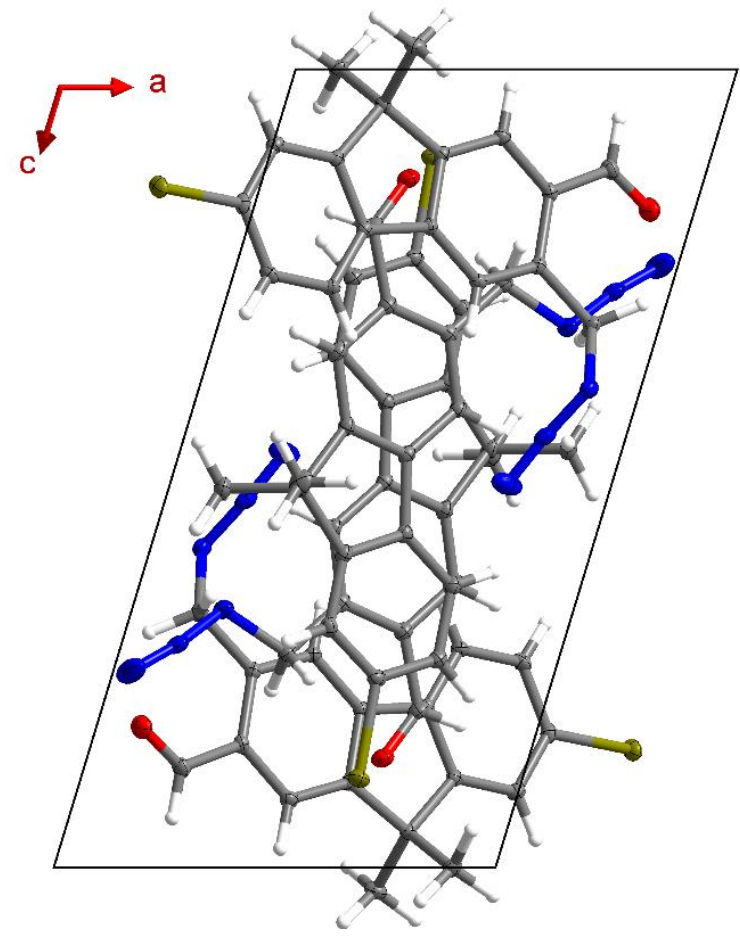

Figure S3: Unit cell packing view of a $\mathbf{5}$ crystal along the $b$-axis. 


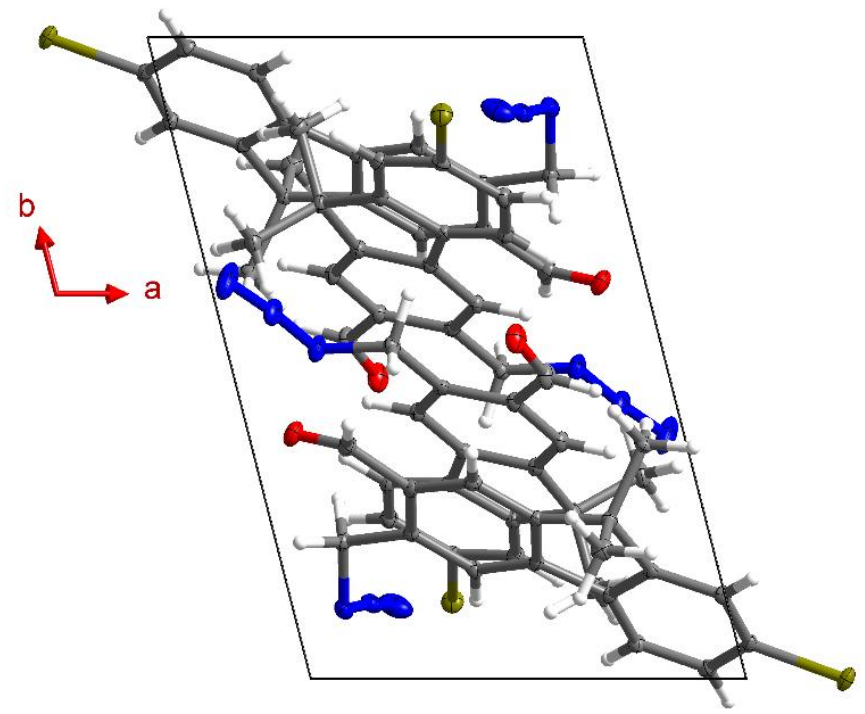

Figure S4: Unit cell packing view of a 5 crystal along the $c$-axis. 


\section{References}

1. Armarego, W. L. F.; Chai, C. L. L. Purification of Laboratory Chemicals, 6th ed.; Elsevier/BH: Oxford, 2009.

2. Sheldrick, G. M. Acta Cryst. A 2008, 64, 112-122. doi:10.1107/S0108767307043930

3. Dolomanov, O. V.; Bourhis, L. J.; Gildea, R. J.; Howard, J. A. K.; Puschmann, H. J. Appl. Cryst. 2009, 42, 339-341. doi:10.1107/S0021889808042726

4. Krasovskiy, A.; Knochel, P. Angew. Chem. Int. Ed. 2004, 43, 3333-3336. doi:10.1002/anie.200454084

5. Bodroux, F. C. R. Chim. 1902, 135, 1350-1351.

6. Krasovskiy, A.; Krasovskaya, V.; Knochel, P. Angew. Chem. Int. Ed. 2006, 45, 2958-2961. doi:10.1002/anie.200504024

7. Hammett, L. P.; Walden, G. H.; Edmonds, S. M. J. Am. Chem. Soc. 1934, 56, 1092-1094. doi:10.1021/ja01320a025

8. Schwekendiek, K.; Glorius, F. Synthesis 2006, 2006, 2996-3002. doi:10.1055/s-2006-950198

9. Batista, J. H. C.; dos Santos, F. M.; Bozzini, L. A.; Vessecchi, R.; Oliveira, A. R. M.; Clososki, G. C. Eur. J. Org. Chem. 2015, 2015, 967-977. doi:10.1002/ejoc.201403255

10. Cheng, P.; Clive, D. L. J. J. Org. Chem. 2012, 77, 3348-3364. doi:10.1021/jo3001657

11. Fischer, D.; Tomeba, H.; Pahadi, N. K.; Patil, N. T.; Yamamoto, Y. Angew. Chem. Int. Ed. 2007, 46, 4764-4766. doi:10.1002/anie.200701392

12. Meyers, A.I.; Willemsen, J. J. Tetrahedron 1998, 54, 10493-10511. doi:10.1016/S00404020(98)00501-8

13. John, J.; Thomas, J.; Parekh, N.; Dehaen, W. Eur. J. Org. Chem. 2015, 2015, 4922-4930. doi:10.1002/ejoc.201500459

14. Ackermann, L.; Barfüsser, S.; Kornhaass, C.; Kapdi, A. R. Org. Lett. 2011, 13, 3082-3085. doi:10.1021/ol200986x

15. Sureshbabu, R.; Saravanan, V.; Dhayalan, V.; Mohanakrishnan, A. K. Eur. J. Org. Chem. 2011, 922-935. doi:10.1002/ejoc.201001309

16. Summers, G. J.; Maseko, R. B.; Beebeejaun, B. M. P.; Summers, C. A. J. Polym. Sci. A Polym. Chem. 2011, 49, 2601-2614. doi:10.1002/pola.24692

17. Tang, X.; Zhang, D.; Jie, S.; Sun, W.-H.; Chen, J. J. Organomet. Chem. 2005, 690, 3918-3928. doi:10.1016/j.jorganchem.2005.05.026

18. Yang, J.; Jiang, C.; Zhang, Y.; Yang, R.; Yang, W.; Hou, Q.; Cao, Y. Macromolecules 2004, 37, 1211-1218. doi:10.1021/ma035743u

19. Chakraborty, C.; Bera, M. K.; Rana, U.; Malik, S. Chem. Commun. 2015, 51, 13123-13126. doi:10.1039/c5cc04275e

20. Singh, V.; Wang, S.; Kool, E. T. J. Am. Chem. Soc. 2013, 135, 6184-6191. doi:10.1021/ja4004393 
21. Hosseinzadeh, R.; Mohadjerani, M.; Pooryousef, M. Luminescence 2015, 30, 549-555. doi:10.1002/bio.2776

22. Denmark, S. E.; Cullen, L. R. J. Org. Chem. 2015, 80, 11818-11848. doi:10.1021/acs.joc.5b01759

23. Göbel, D.; Clamor, N.; Nachtsheim, B. J. Org. Biomol. Chem. 2018, 16, 4071-4075. doi:10.1039/c8ob01072b

24. Meyers, A. I.; Avila, W. B. J. Org. Chem. 1981, 46, 3881-3886. doi:10.1021/jo00332a024

25. Bos, M. E.; Wulff, W. D.; Miller, R. A.; Chamberlin, S.; Brandvold, T. A. J. Am. Chem. Soc. 1991, 113, 9293-9319. doi:10.1021/ja00024a040

26. Lingel, A.; Sendzik, M.; Huang, Y.; Shultz, M. D.; Cantwell, J.; Dillon, M. P.; Fu, X.; Fuller, J.; Gabriel, T.; Gu, J.; Jiang, X.; Li, L.; Liang, F.; McKenna, M.; Qi, W.; Rao, W.; Sheng, X.; Shu, W.; Sutton, J.; Taft, B.; Wang, L.; Zeng, J.; Zhang, H.; Zhang, M.; Zhao, K.; Lindvall, M.; Bussiere, D. E. J. Med. Chem. 2017, 60, 415-427. doi:10.1021/acs.jmedchem.6b01473

27. Bedford, R. B.; Brenner, P. B.; Carter, E.; Gallagher, T.; Murphy, D. M.; Pye, D. R. Organometallics 2014, 33, 5940-5943. doi:10.1021/om500847j

28. Moon, D. J.; Al-Amin, M.; Lewis, R. S.; Arnold, K. M.; Yap, G. P. A.; Sims-Mourtada, J.; Chain, W. J. Eur. J. Org. Chem. 2018, 3348-3351. doi:10.1002/ejoc.201800707

29. Reddy, B. R. P.; Chowdhury, S.; Auffrant, A.; Gosmini, C. Adv. Synth. Catal. 2018, 360, 30263029. doi:10.1002/adsc. 201800468

30. Stulgies, B.; Prinz, P.; Magull, J.; Rauch, K.; Meindl, K.; Rühl, S.; Meijere, A. de. Chem. Eur. J. 2004, 11, 308-320. doi:10.1002/chem.200400723

31. Newman, S. G.; Lautens, M. J. Am. Chem. Soc. 2010, 132, 11416-11417. doi:10.1021/ja1052335

32. Karamé, I.; Jahjah, M.; Messaoudi, A.; Tommasino, M.L.; Lemaire, M. Tetrahedron: Asymmetry 2004, 15, 1569-1581. doi:10.1016/j.tetasy.2004.03.023

33. Philp, D.; Gramlich, V.; Seiler, P.; Diederich, F. J. Chem. Soc., Perkin Trans. 2 1995, 32, 875886. doi:10.1039/P29950000875

34. Rostovtsev, V. V.; Green, L. G.; Fokin, V. V.; Sharpless, K. B. Angew. Chem. Int. Ed. 2002, 41, 2596-2599. doi:10.1002/1521-3773(20020715)41:14<2596:AID-ANIE2596>3.0.CO;2-4

35. Schmid, J.; Frey, W.; Peters, R. Organometallics 2017, 36, 4313-4324. doi:10.1021/acs.organomet.7b00729 
6 NMR-Spectra

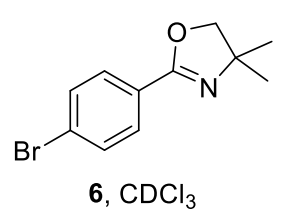

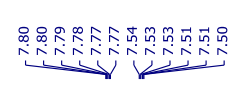

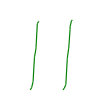

$\stackrel{8}{i}$

$\stackrel{0}{7}$

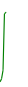

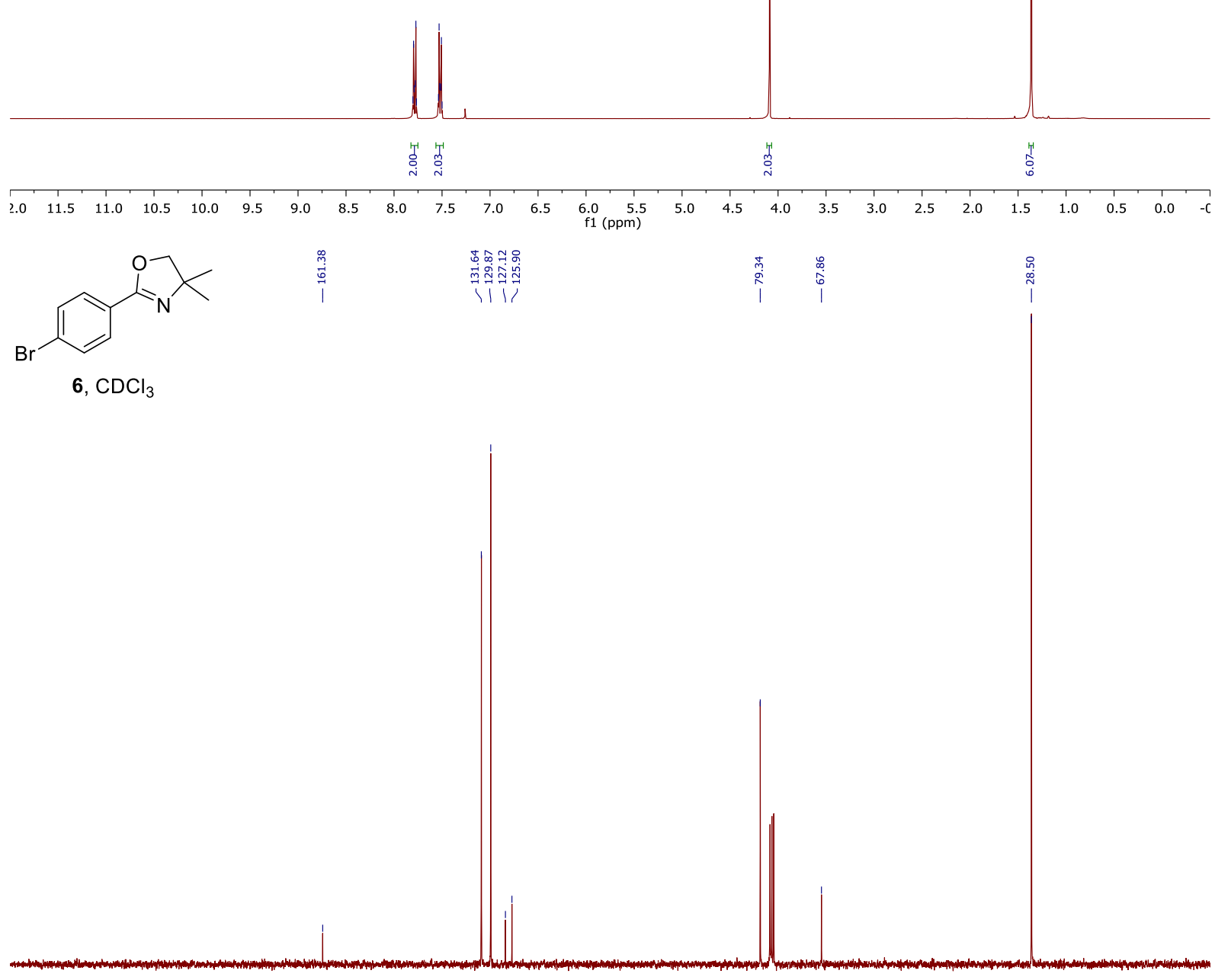

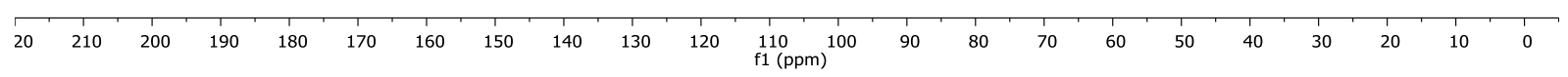

S74 

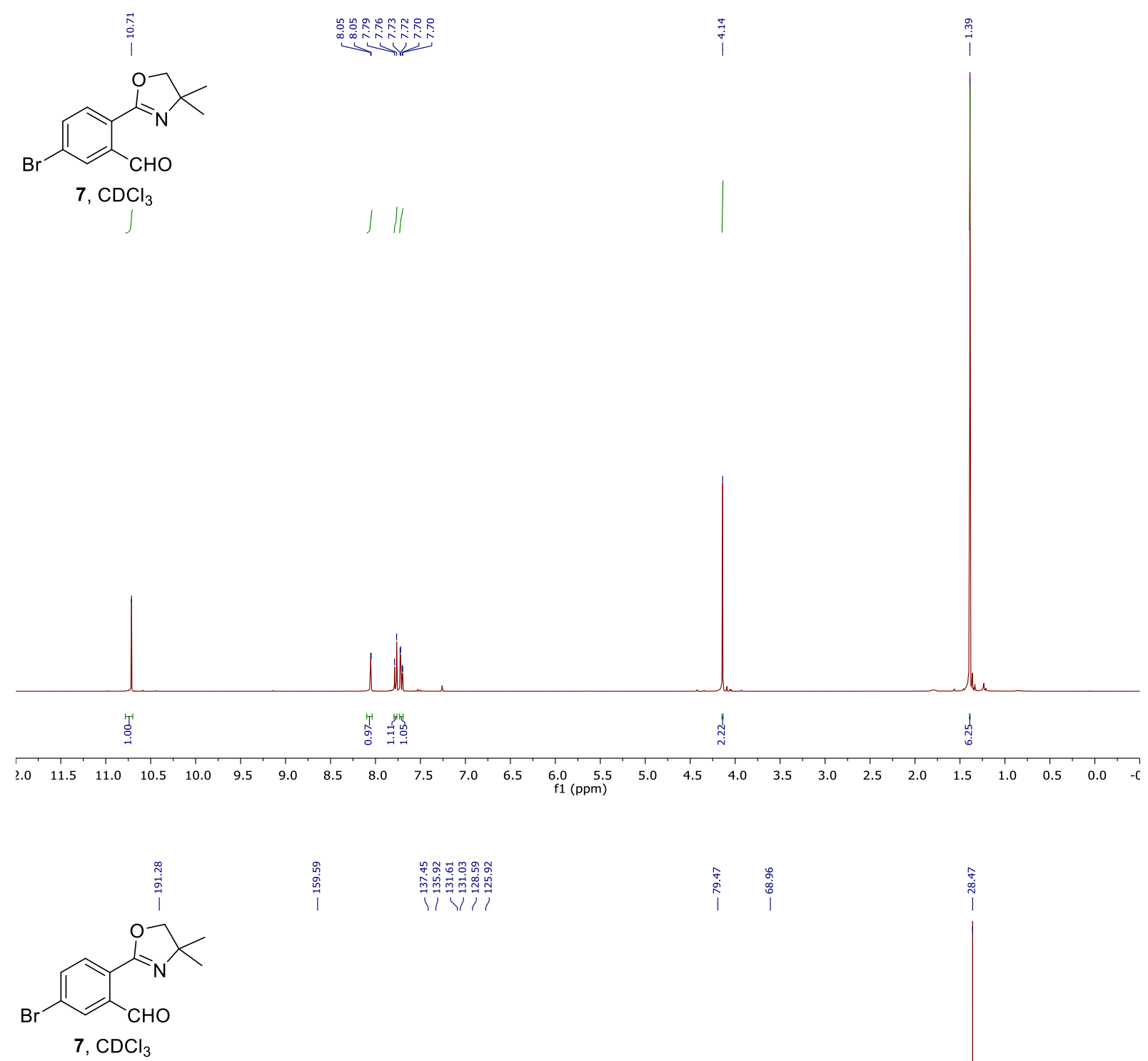

i

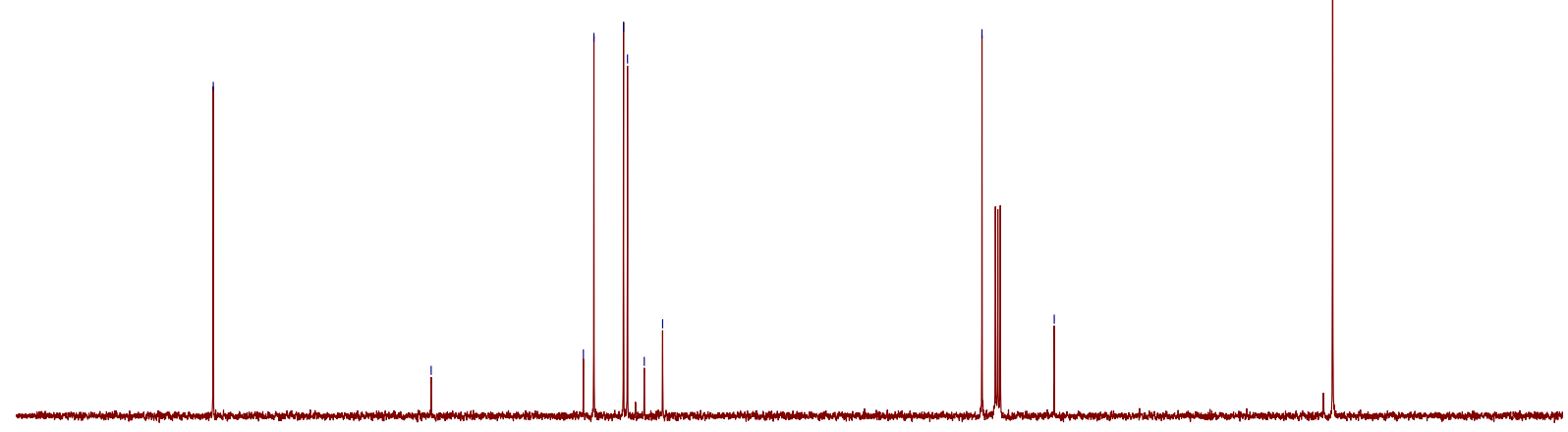

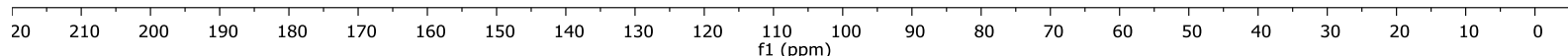



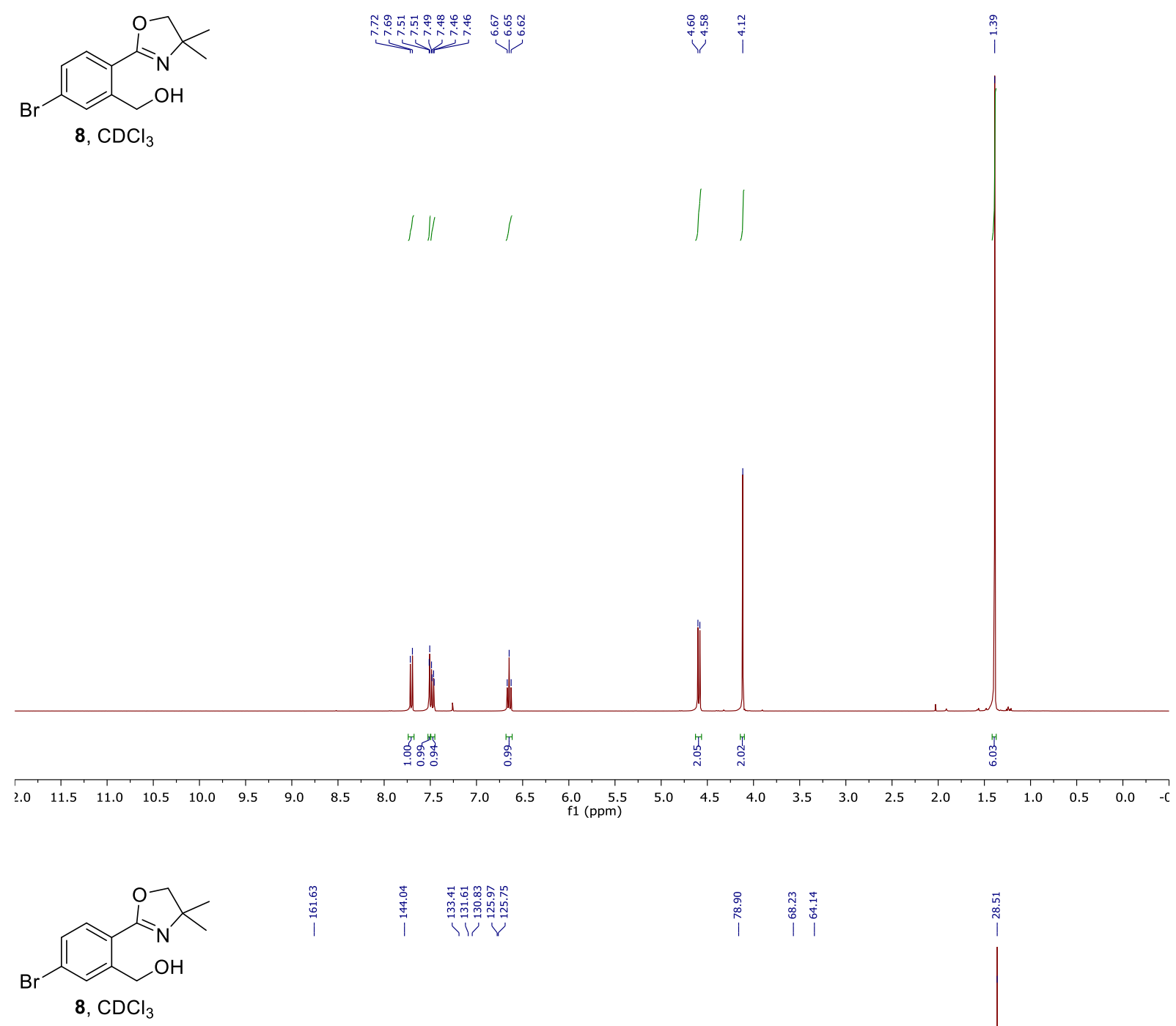

8, $\mathrm{CDCl}_{3}$

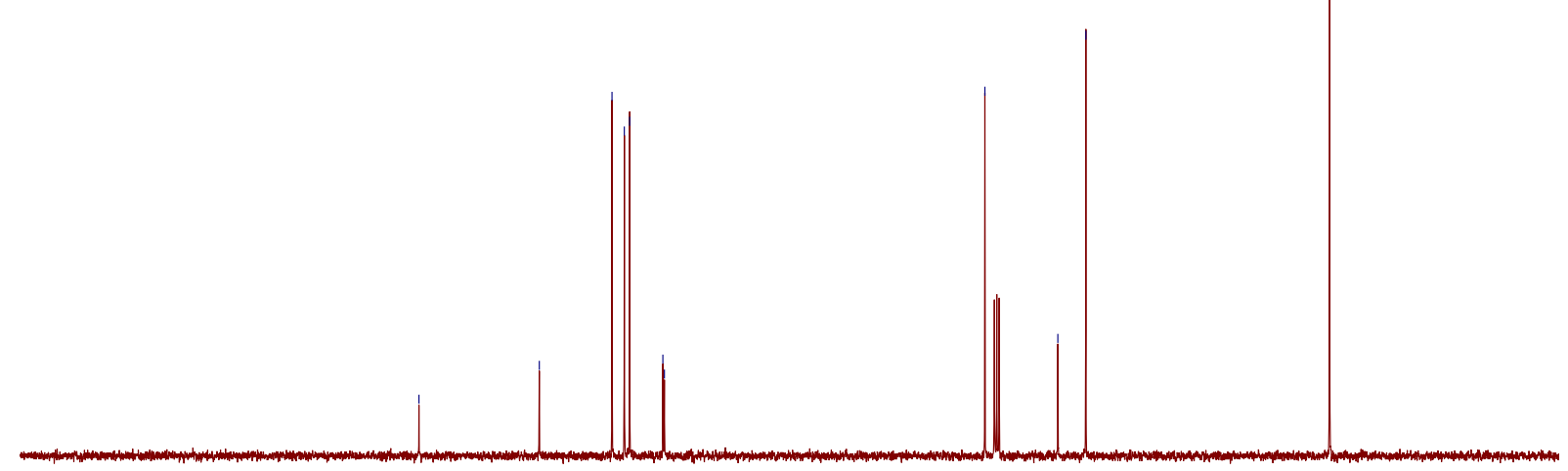

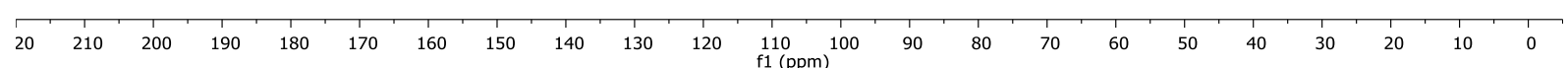




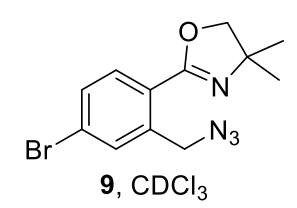

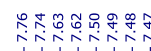

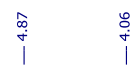

$\stackrel{\hat{m}}{i}$
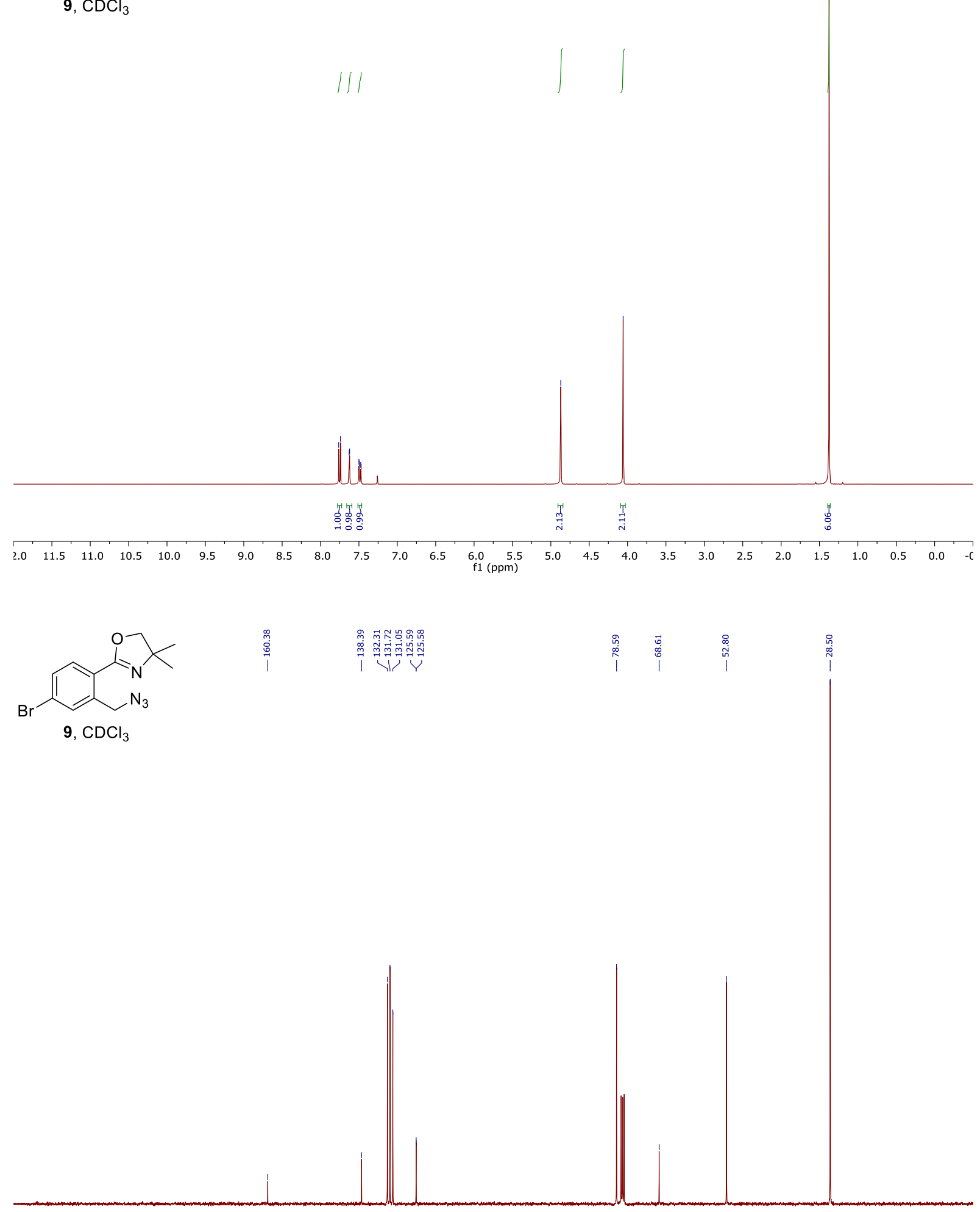

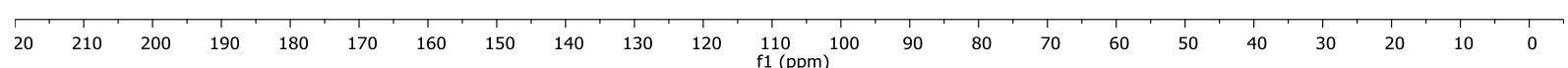



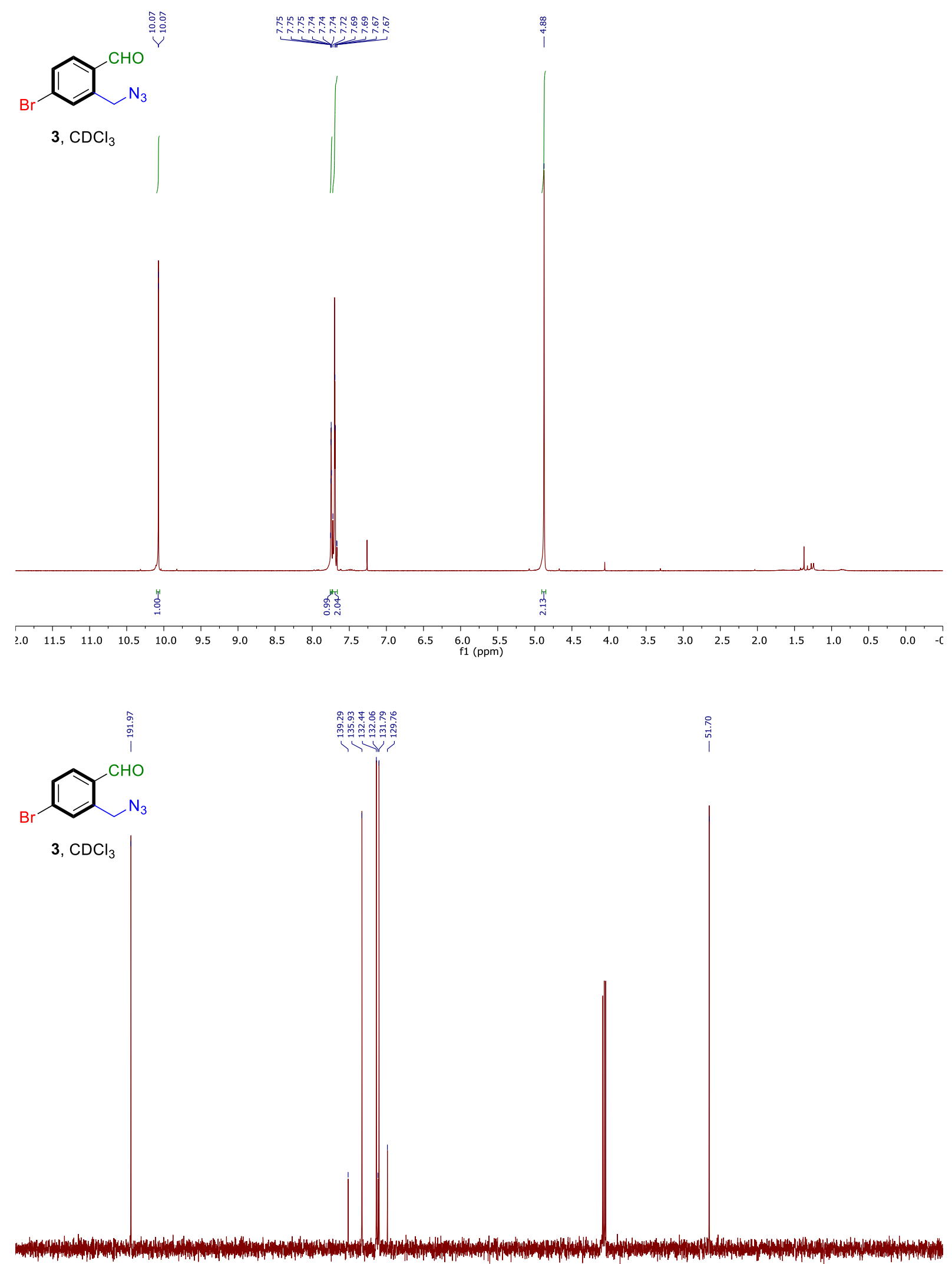

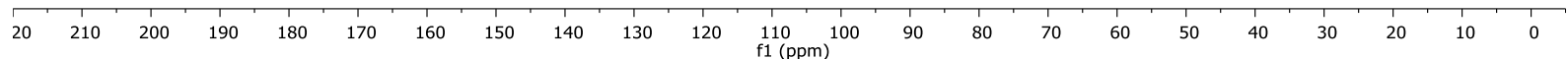



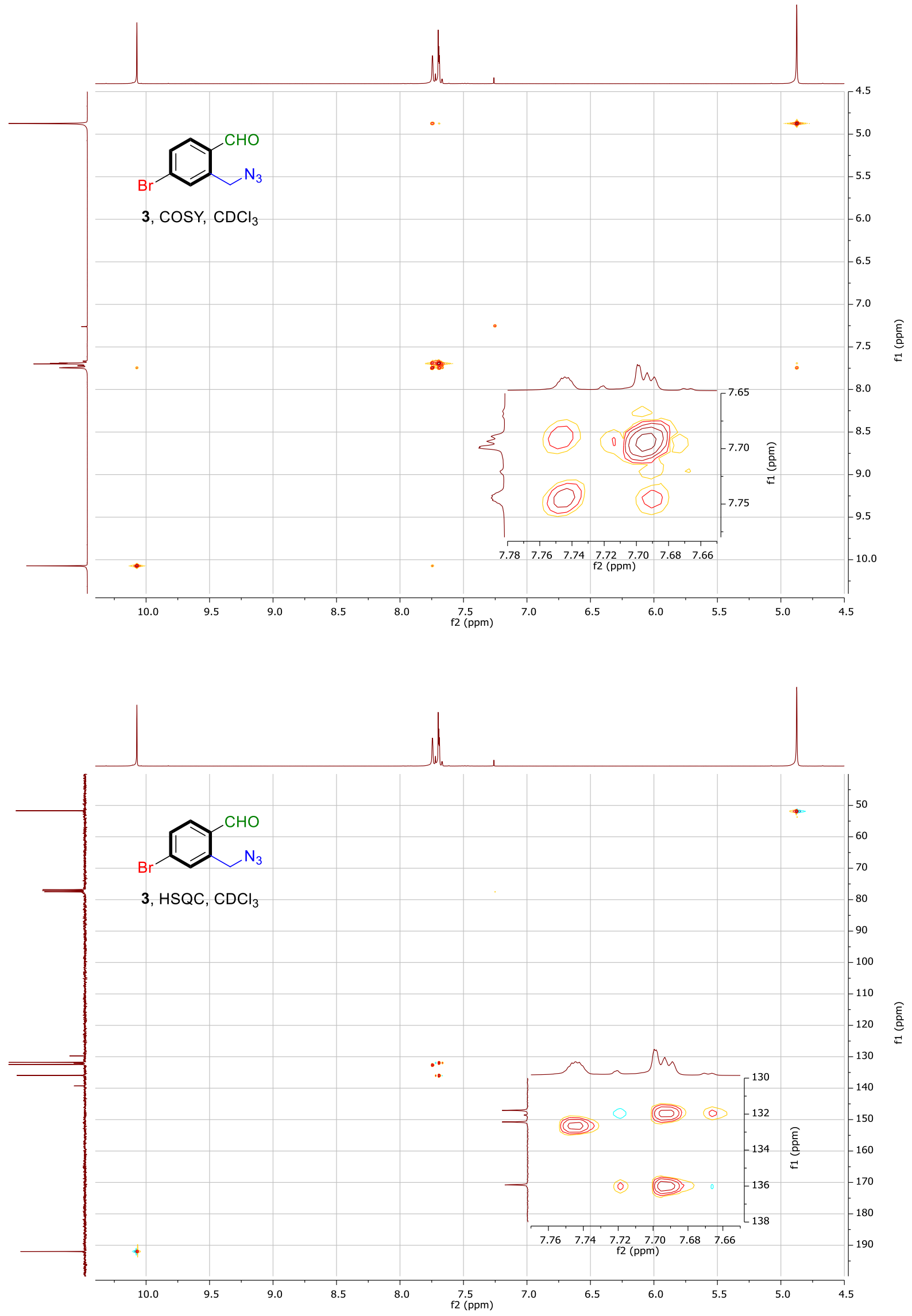


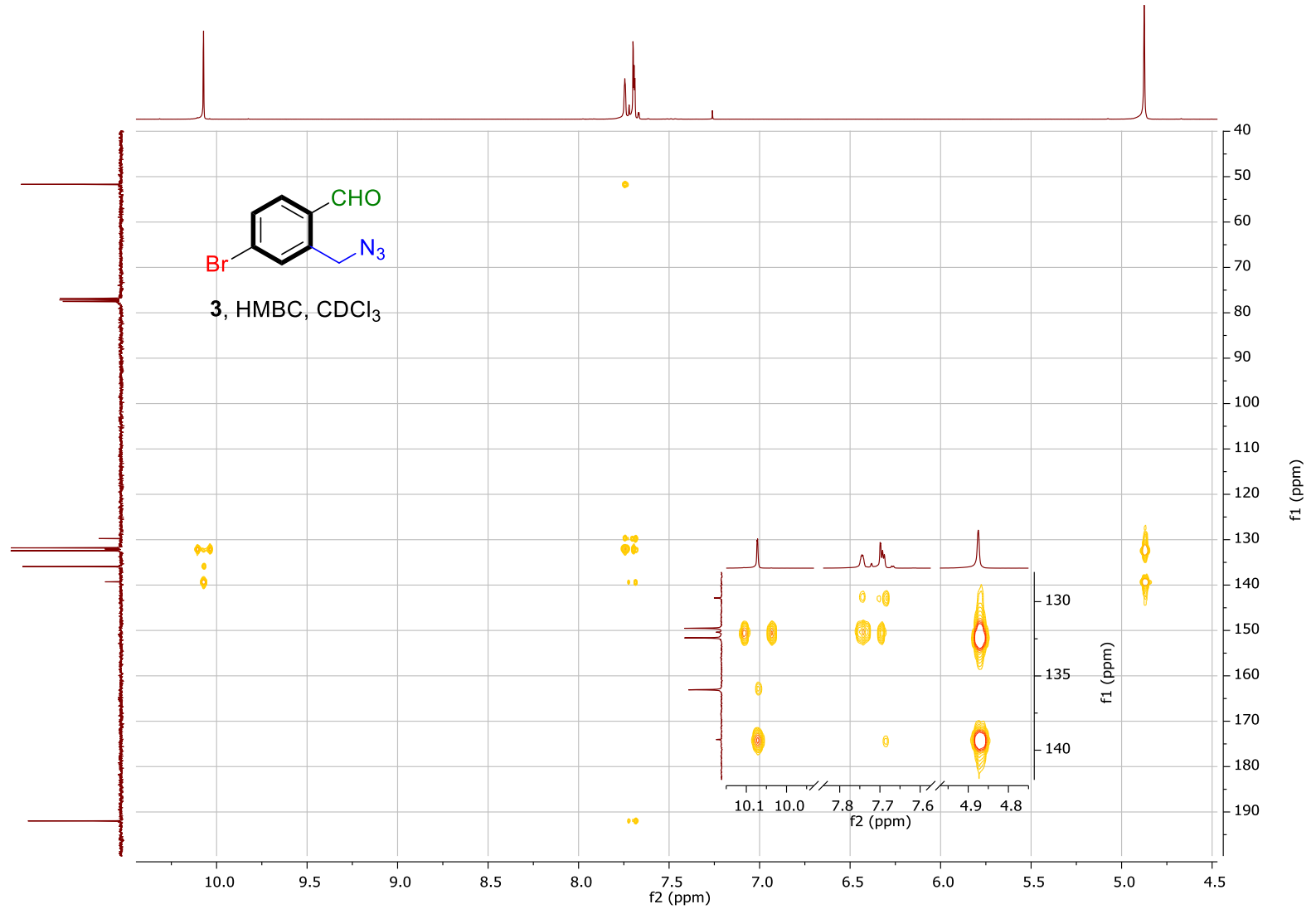



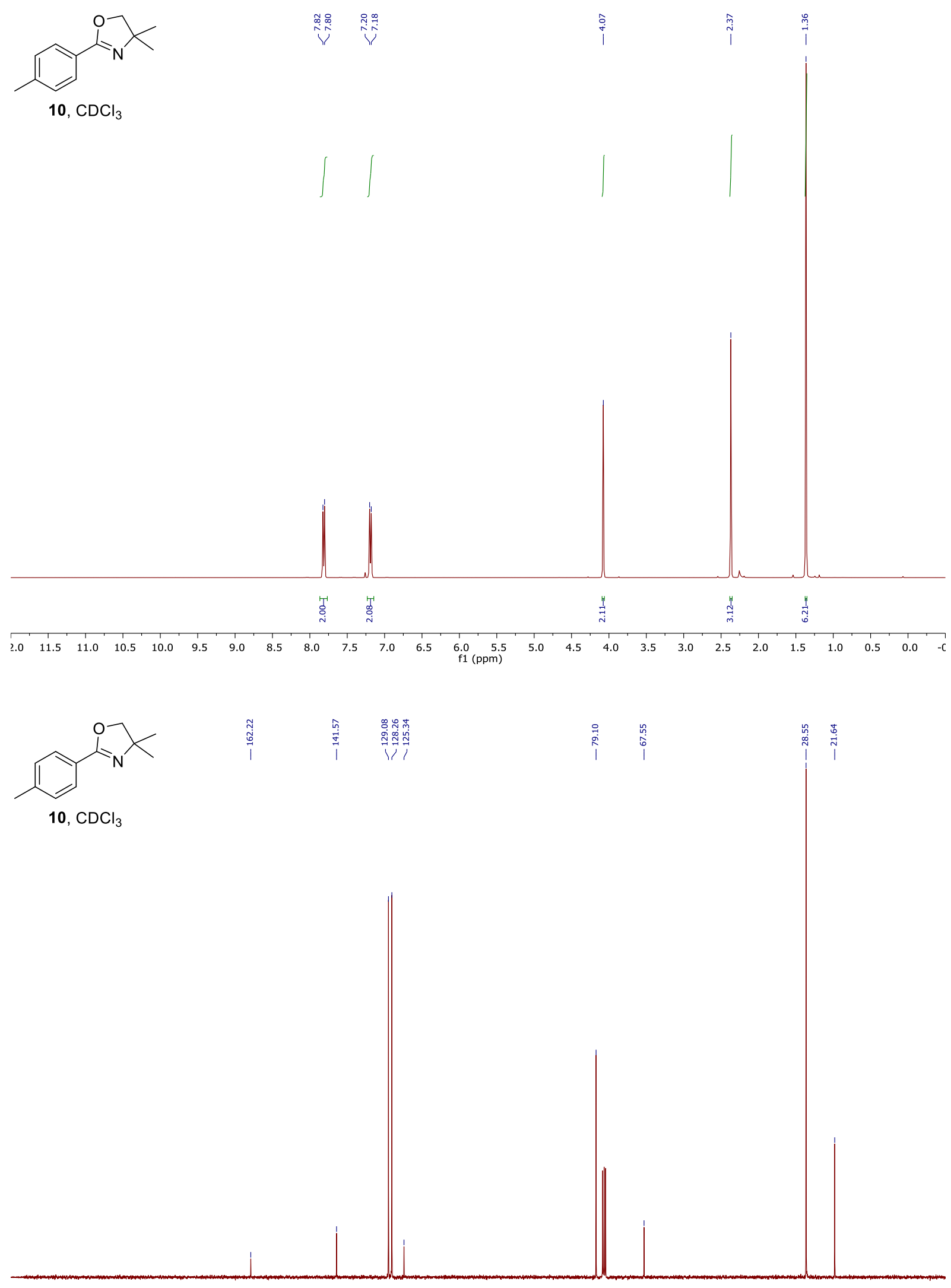

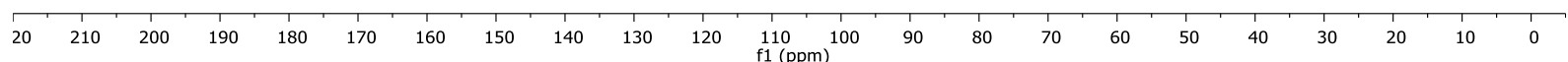



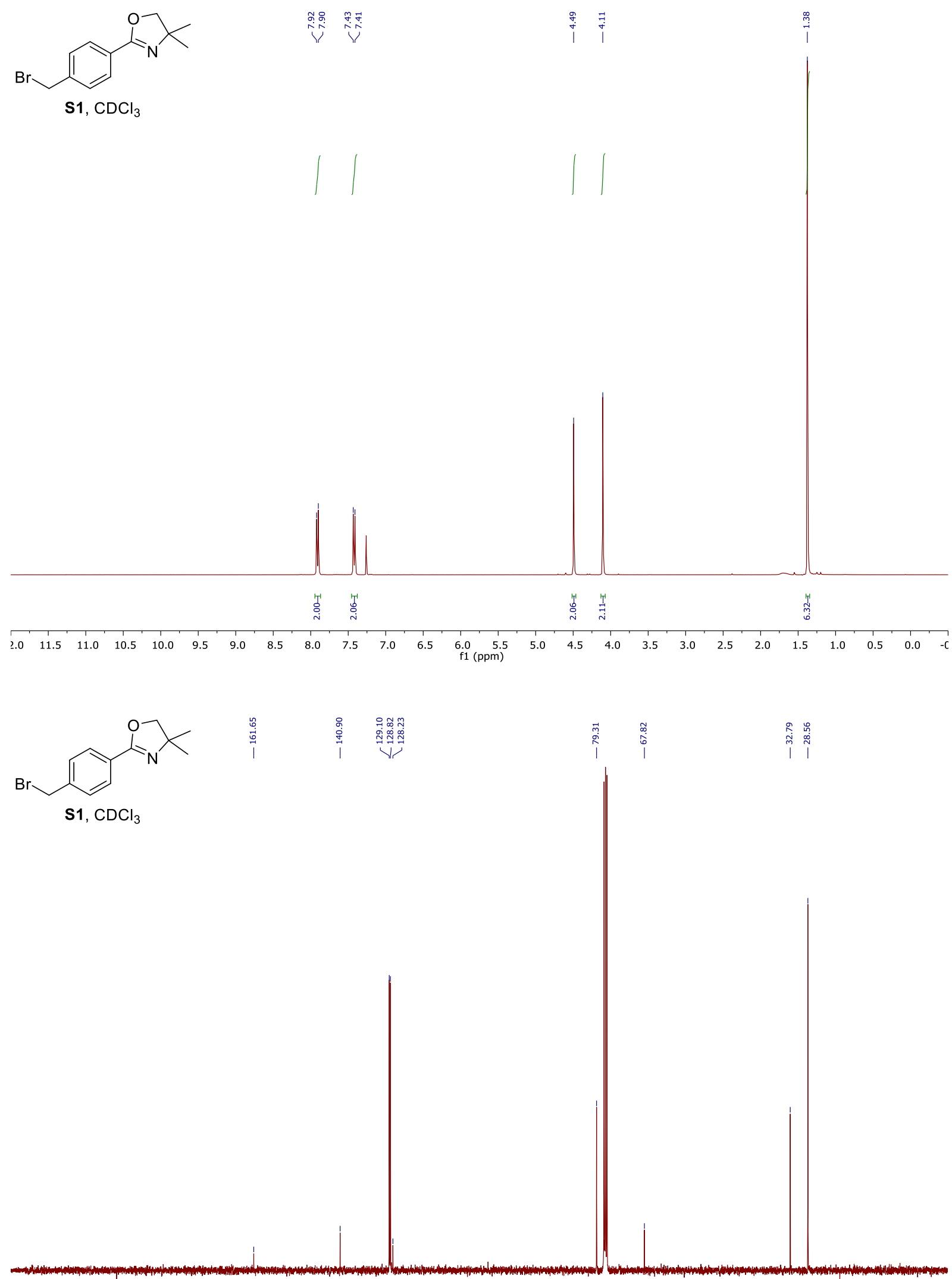

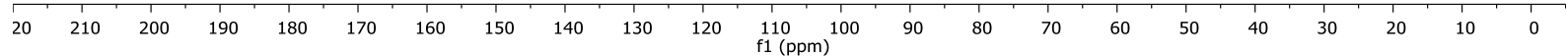




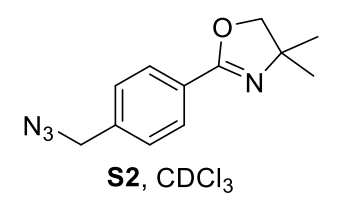

$$
\text { গু }
$$

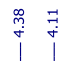

s2, $\mathrm{CDCl}_{3}$

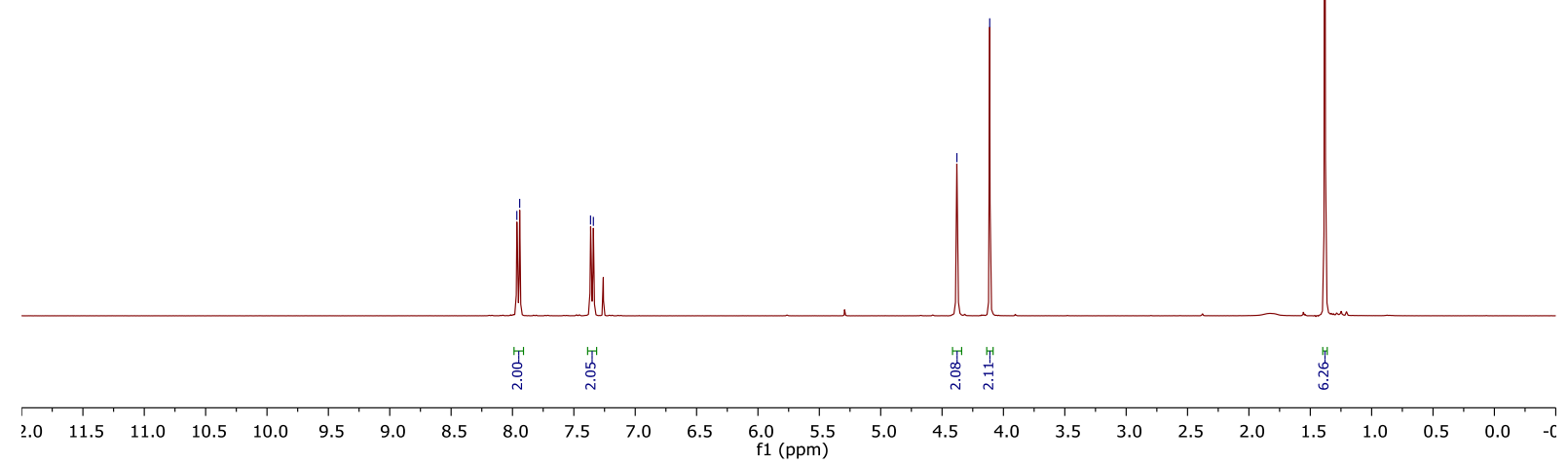

S2, $\mathrm{CDCl}_{3}$

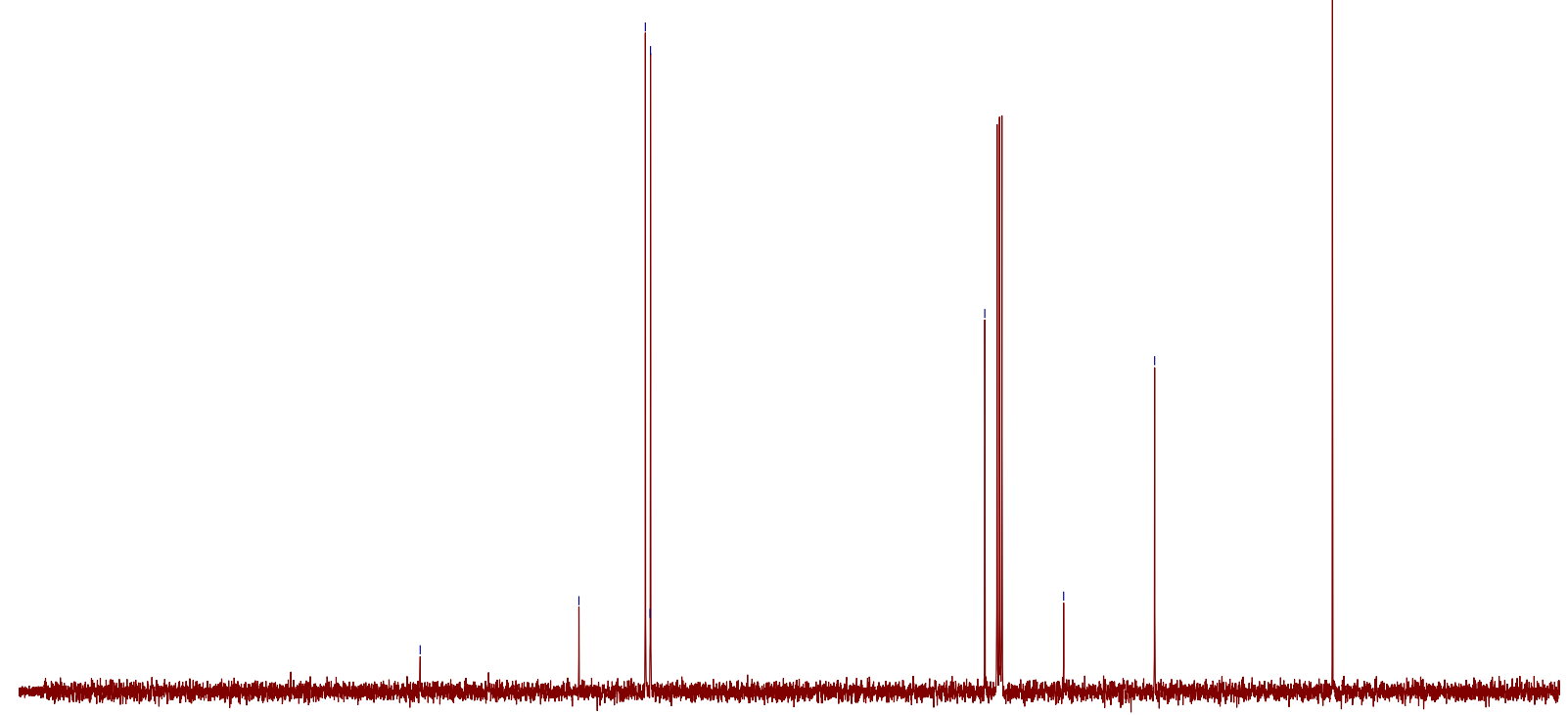

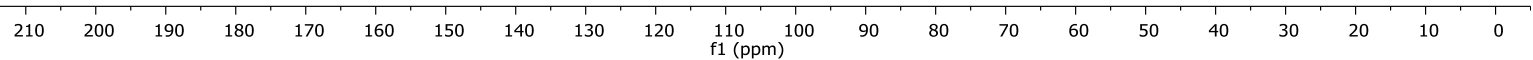




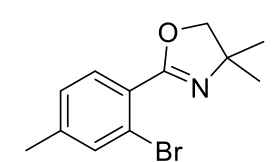

$11, \mathrm{CDCl}_{3}$

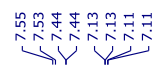

$\stackrel{7}{7}$

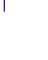

i

|I I

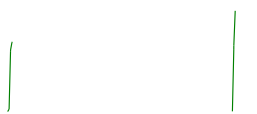

UW
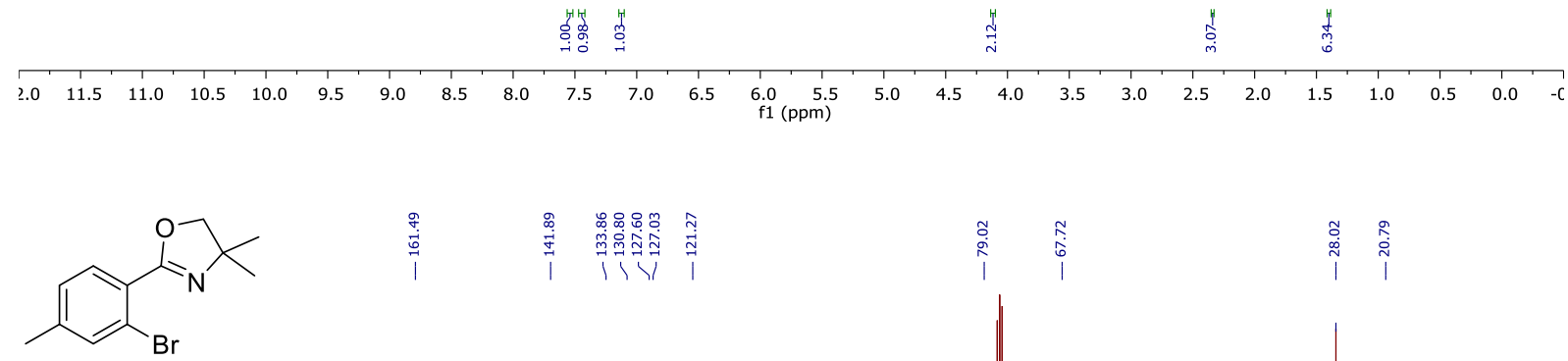

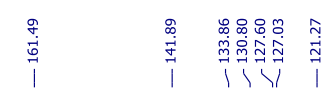

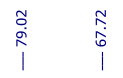

$\stackrel{\substack{0 \\ \infty}}{\stackrel{2}{\sim}}$

$11, \mathrm{CDCl}_{3}$

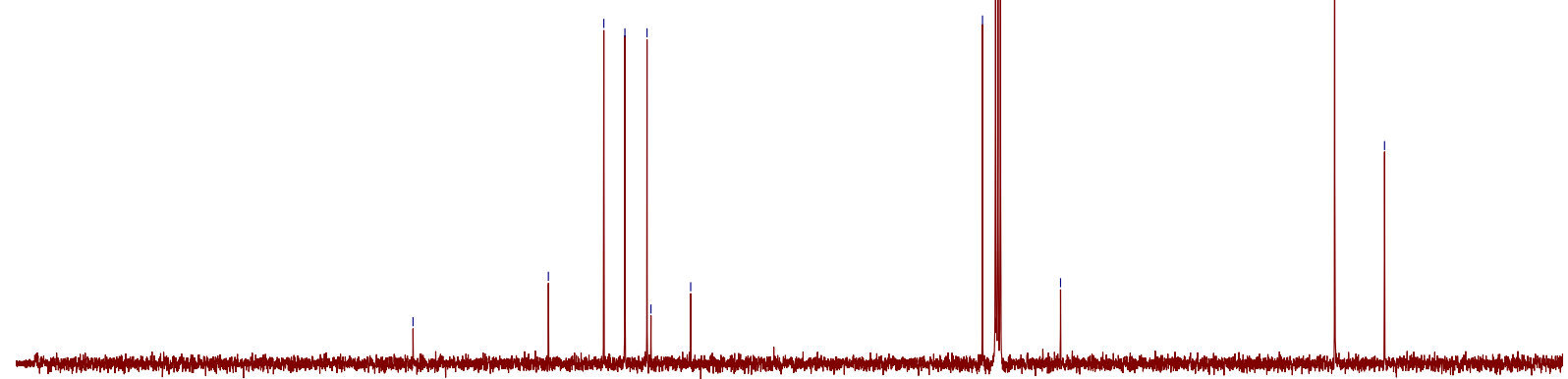

$\begin{array}{lllllllllll}210 & 200 & 190 & 180 & 170 & 160 & 150 & 140 & 130 & 120 & \begin{array}{l}110 \\ \mathrm{f} 1(\mathrm{ppm})\end{array}\end{array}$

90

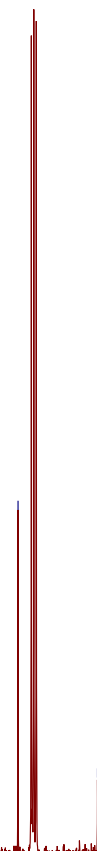




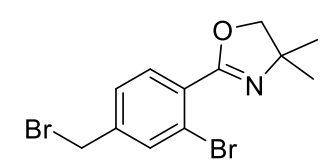

12, $\mathrm{CDCl}_{3}$
妾

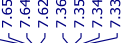

11

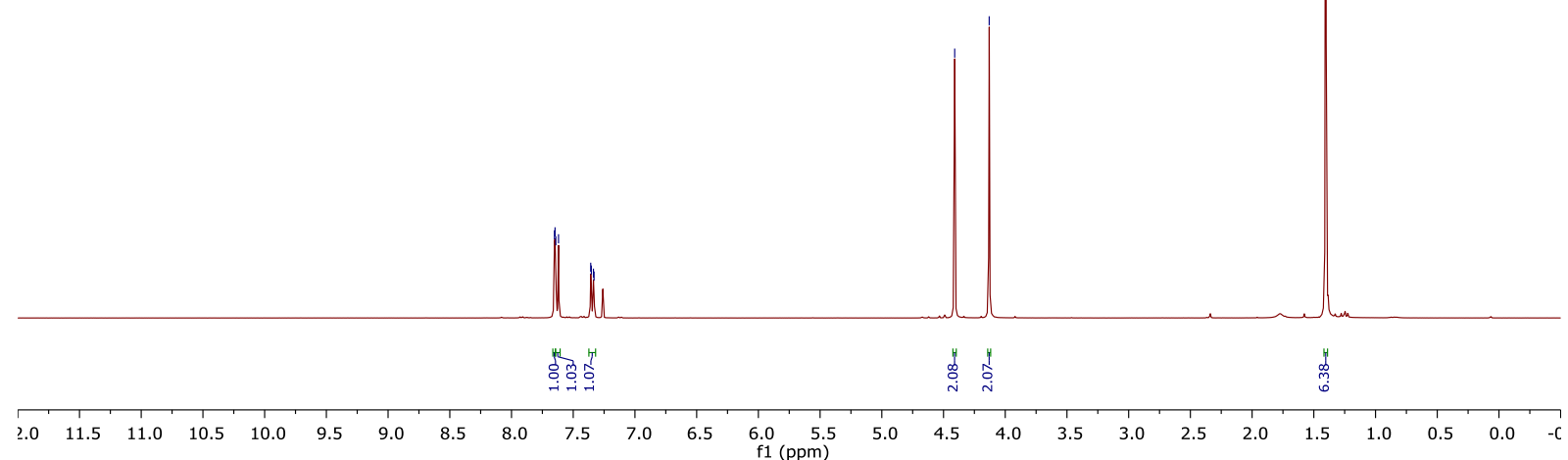

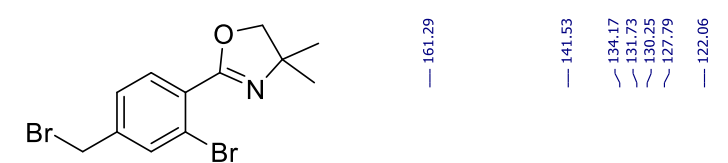

12, $\mathrm{CDCl}_{3}$

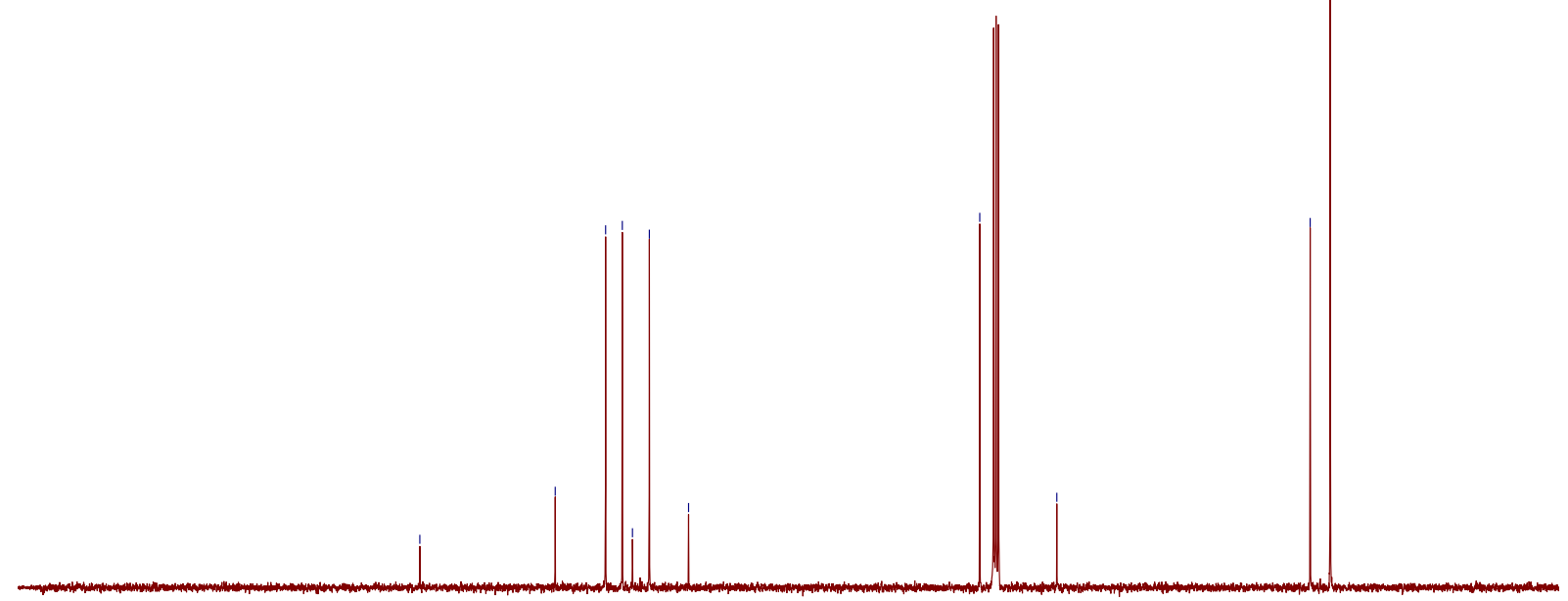

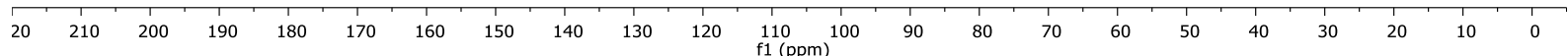




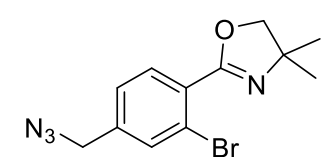

13, $\mathrm{CDCl}_{3}$

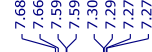

学椋$$
\text { 1 }
$$

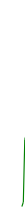

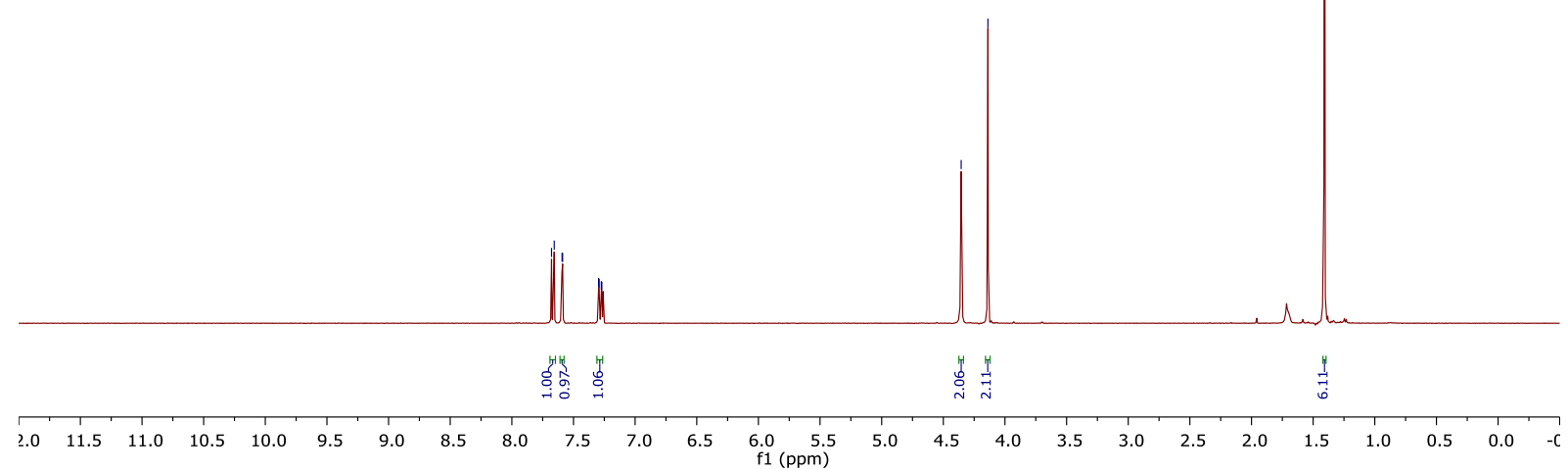

13, $\mathrm{CDCl}_{3}$

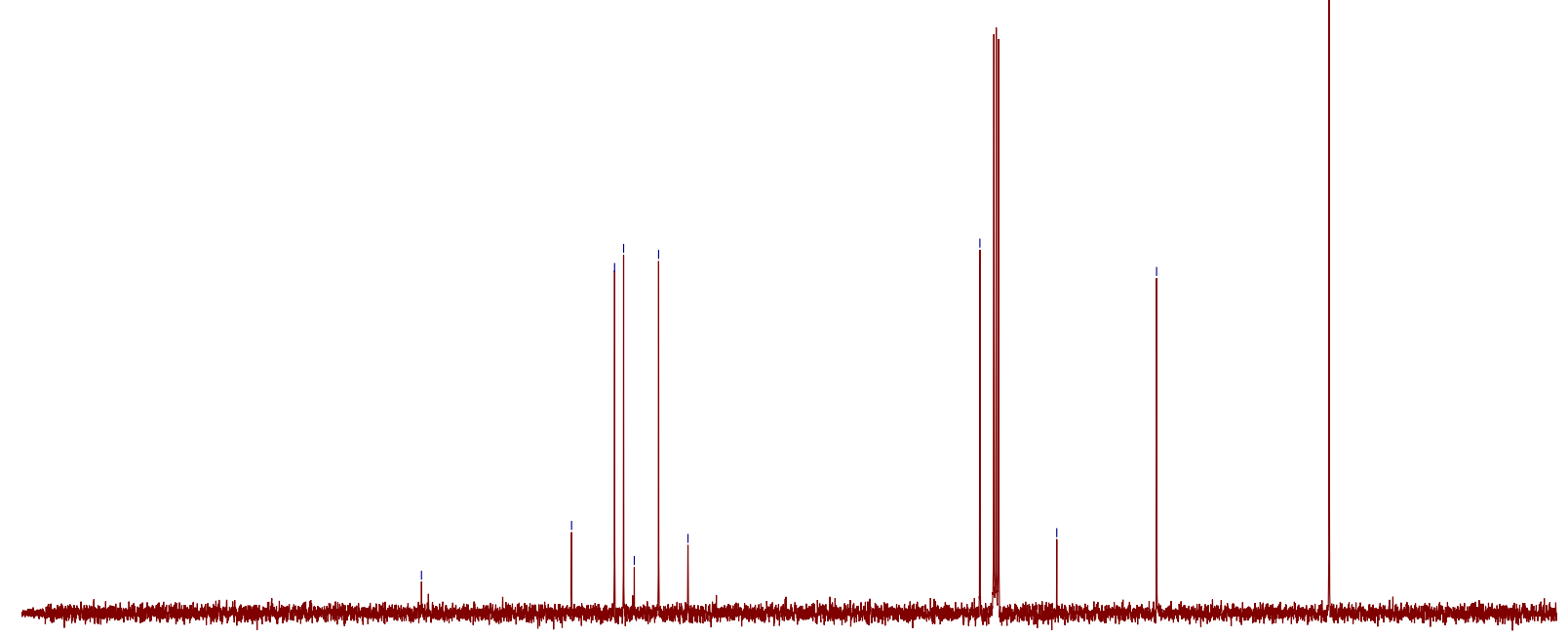




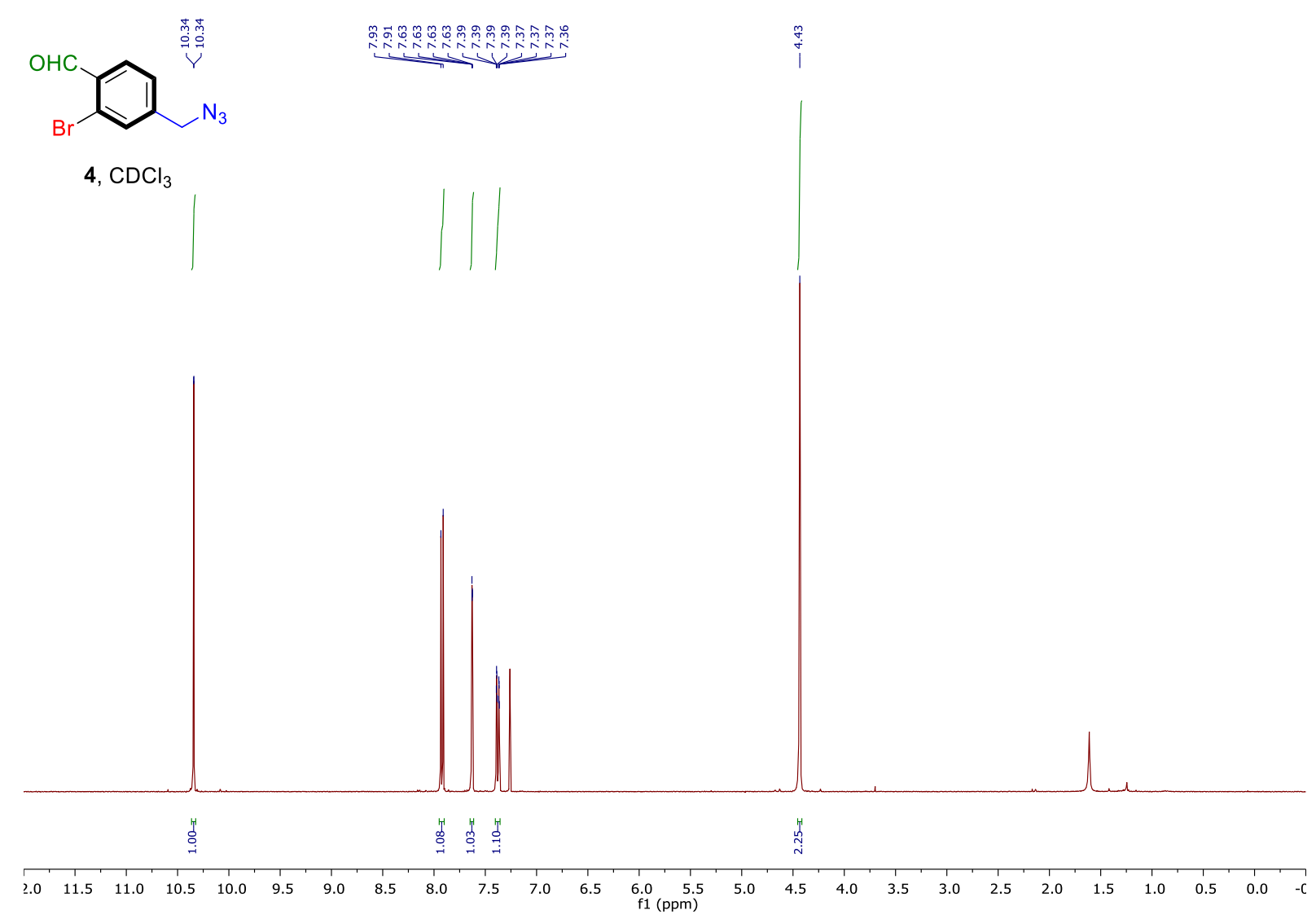

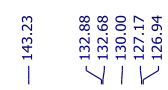

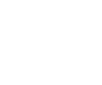

4, $\mathrm{CDCl}_{3}$

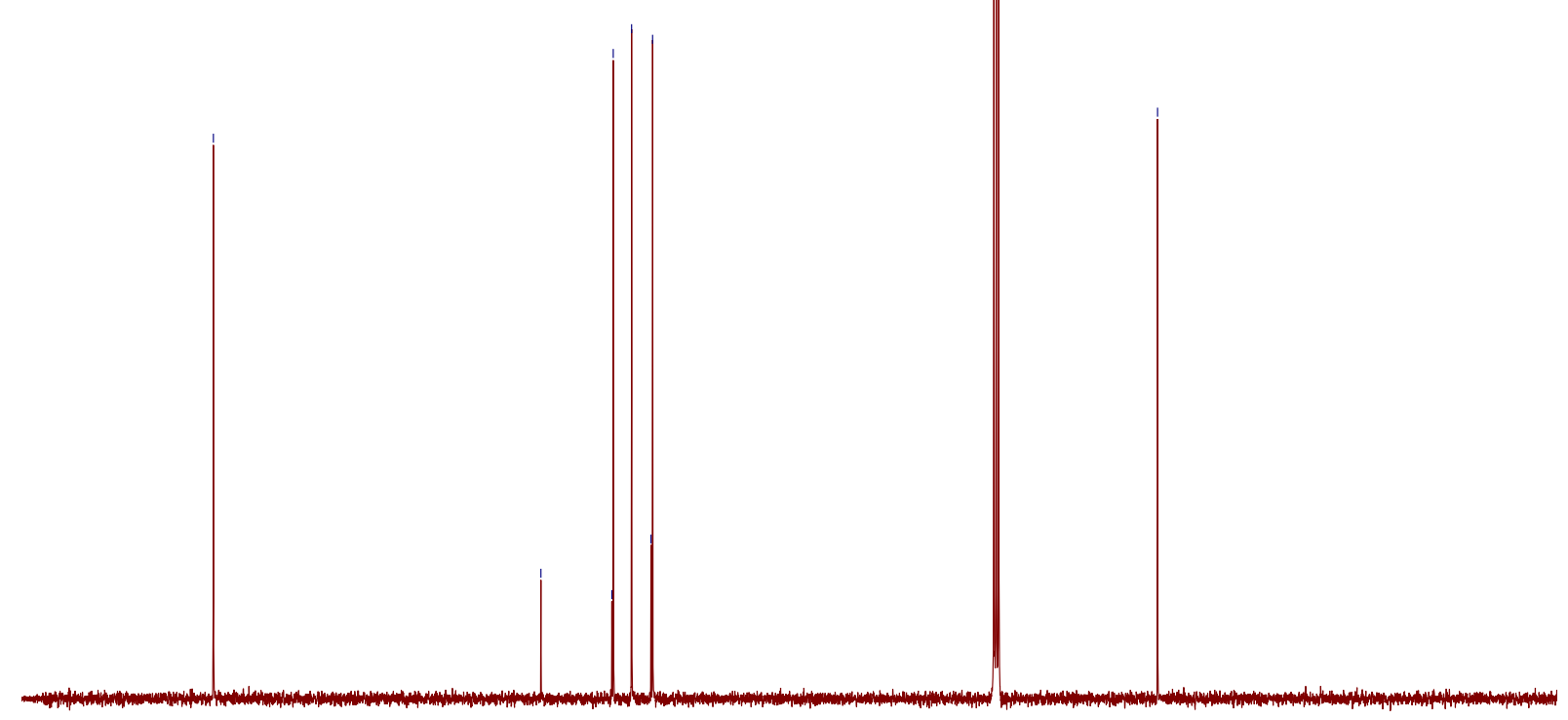

$\begin{array}{lllllllllll}210 & 200 & 190 & 180 & 170 & 160 & 150 & 140 & 130 & 120 & \begin{array}{l}110 \\ \mathrm{f} 1(\mathrm{ppm})\end{array}\end{array}$

90

80

$60 \quad 50$

$$
\text { ( }
$$



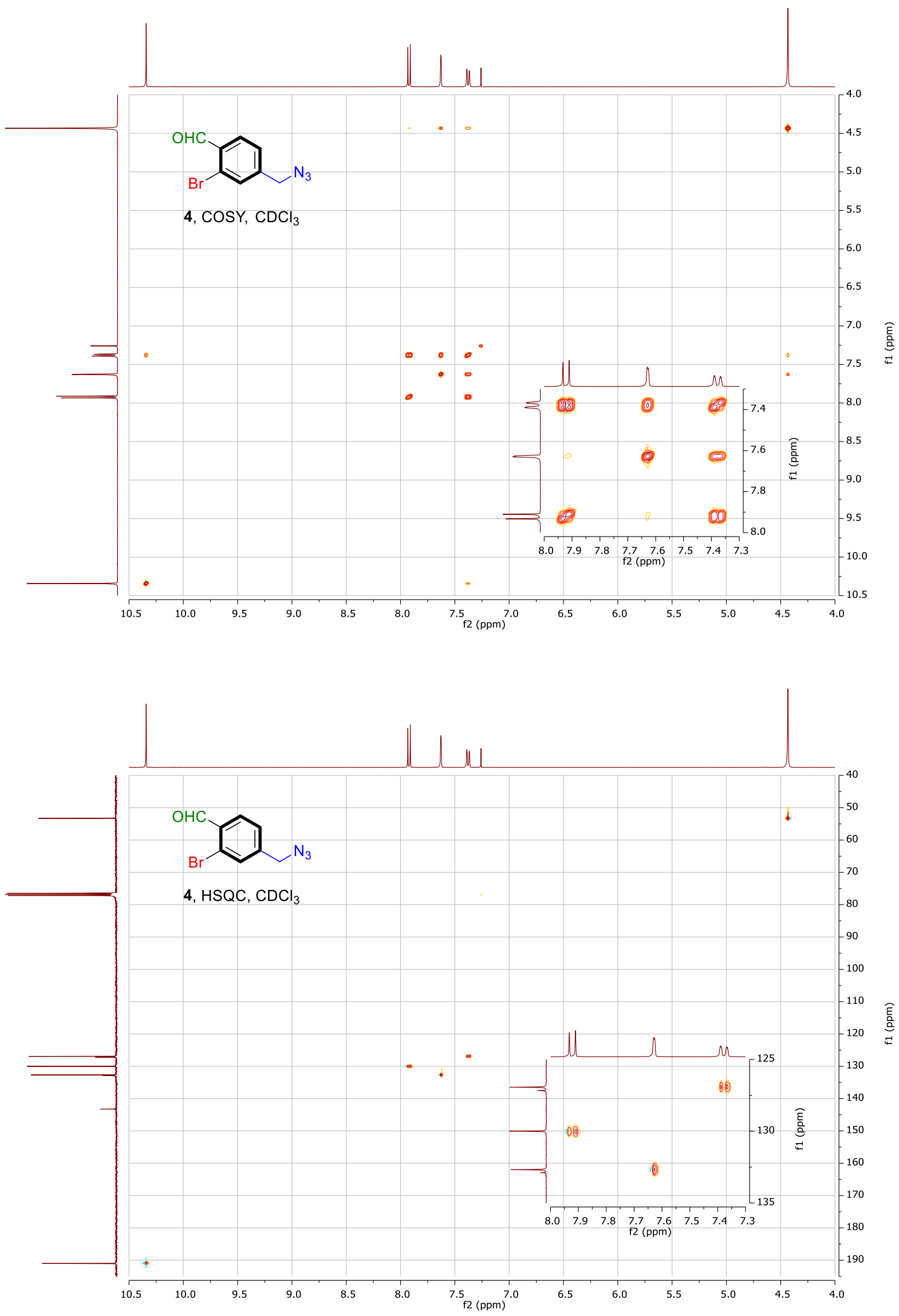

S88 


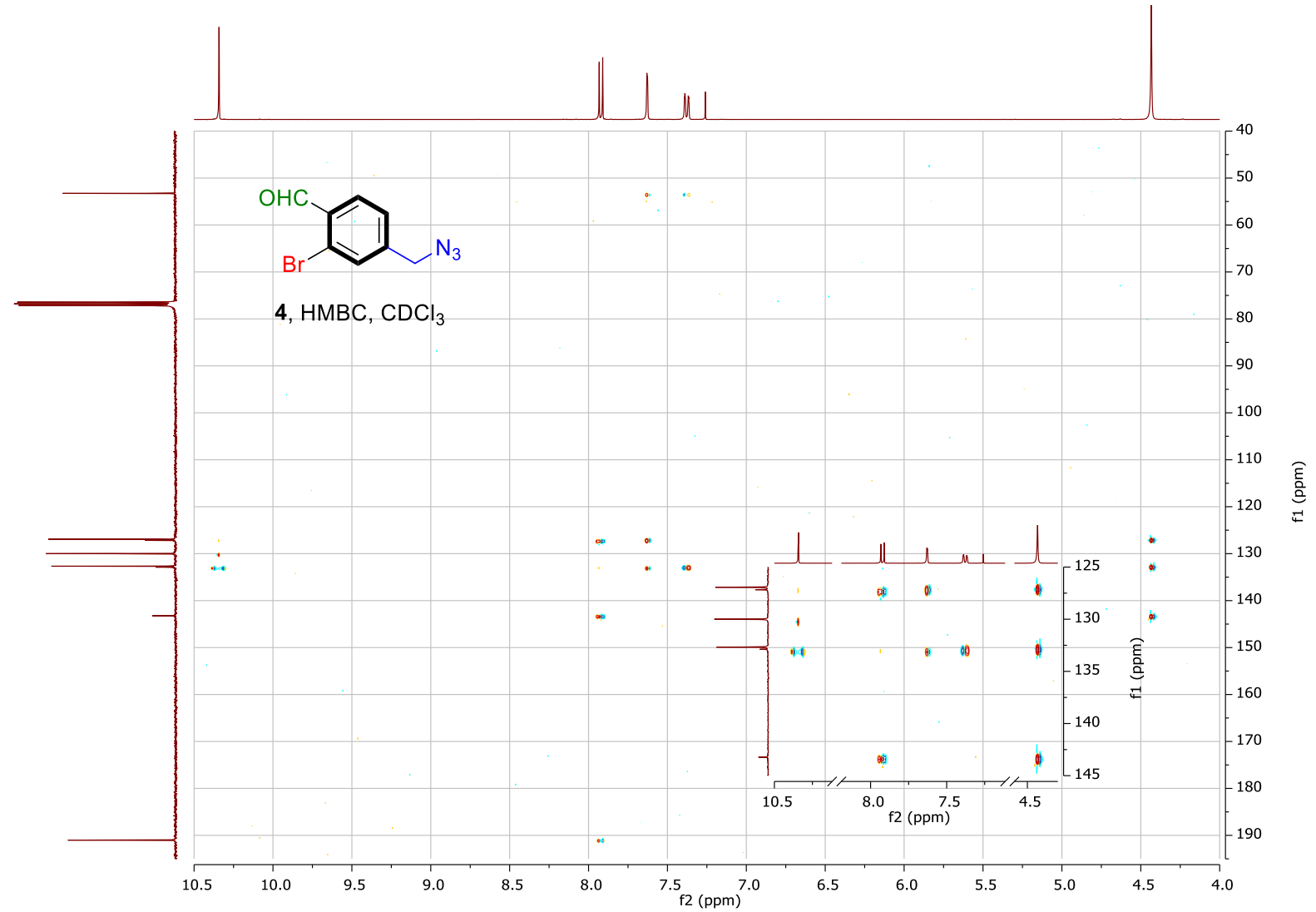



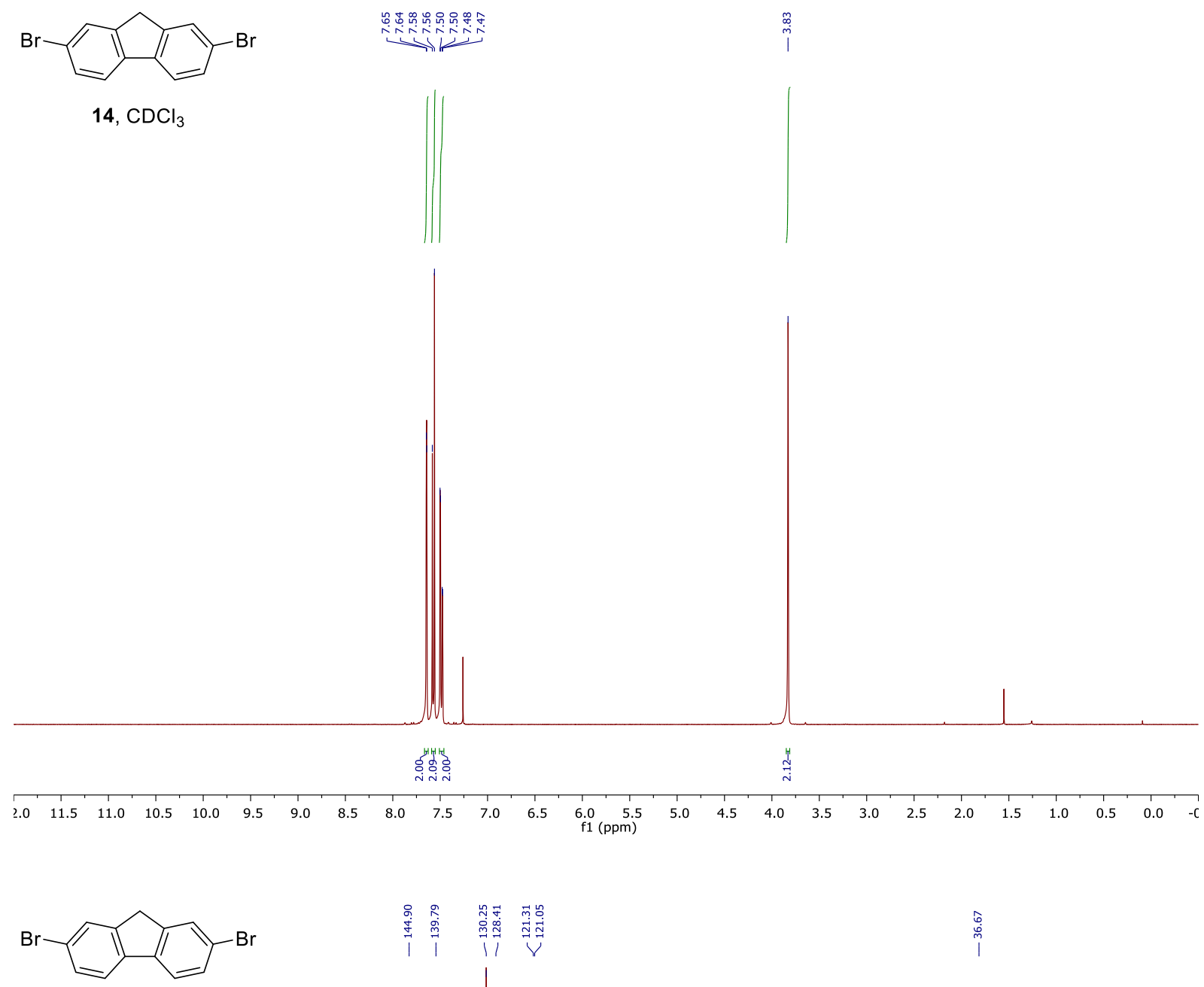

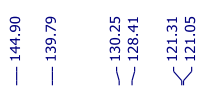

14, $\mathrm{CDCl}_{3}$

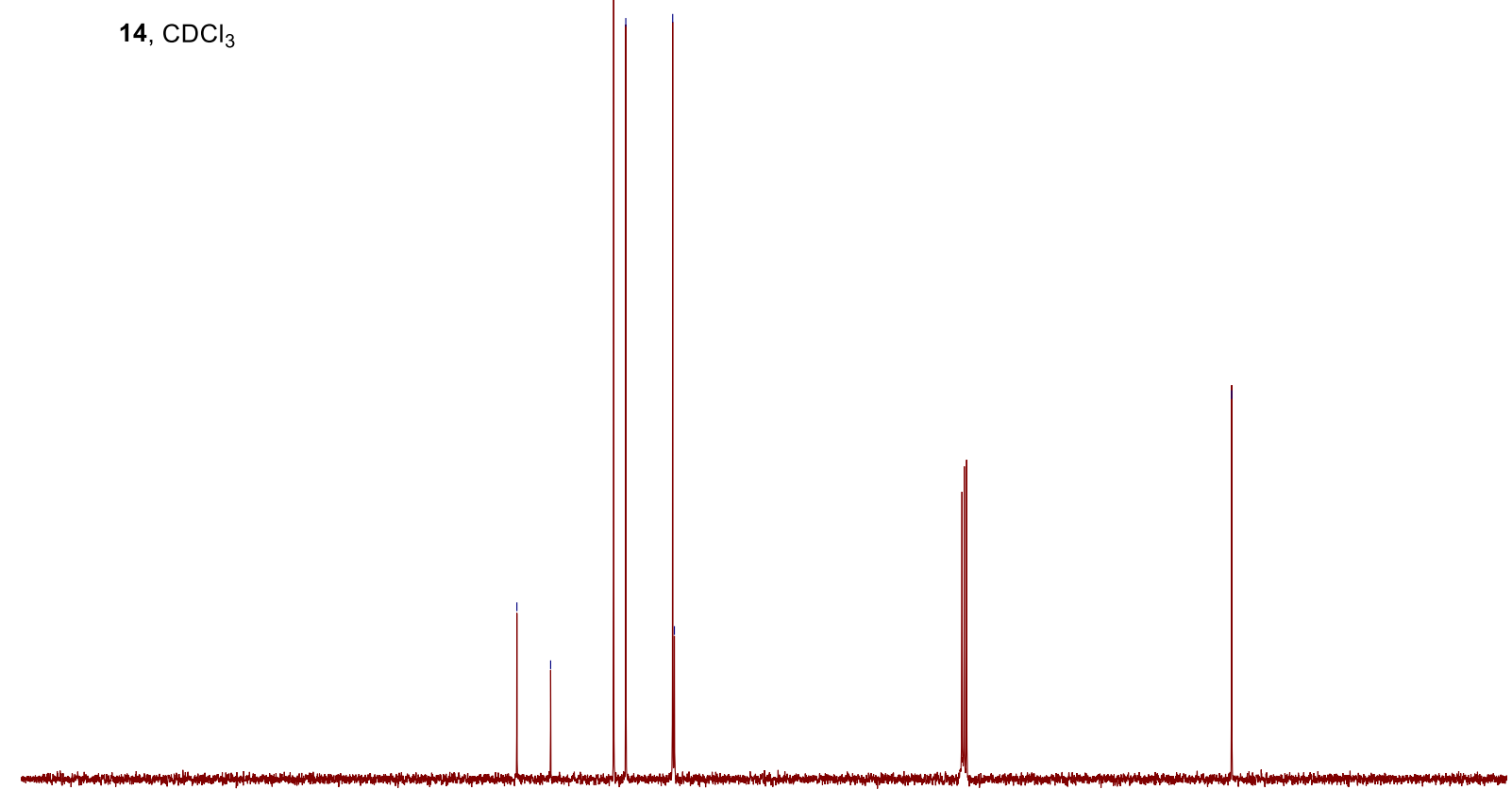

\begin{tabular}{lllllllllllllllllllllllll}
\hline 20 & 210 & 200 & 190 & 180 & 170 & 160 & 150 & 140 & 130 & 120 & $\underset{\mathrm{f} 1(\mathrm{ppm})}{110}$ & 100 & 90 & 80 & 70 & 60 & 50 & 40 & 30 & 20 & 10 & 0
\end{tabular} 


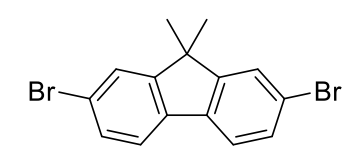

资

$\stackrel{f}{i}$

15, $\mathrm{CDCl}_{3}$
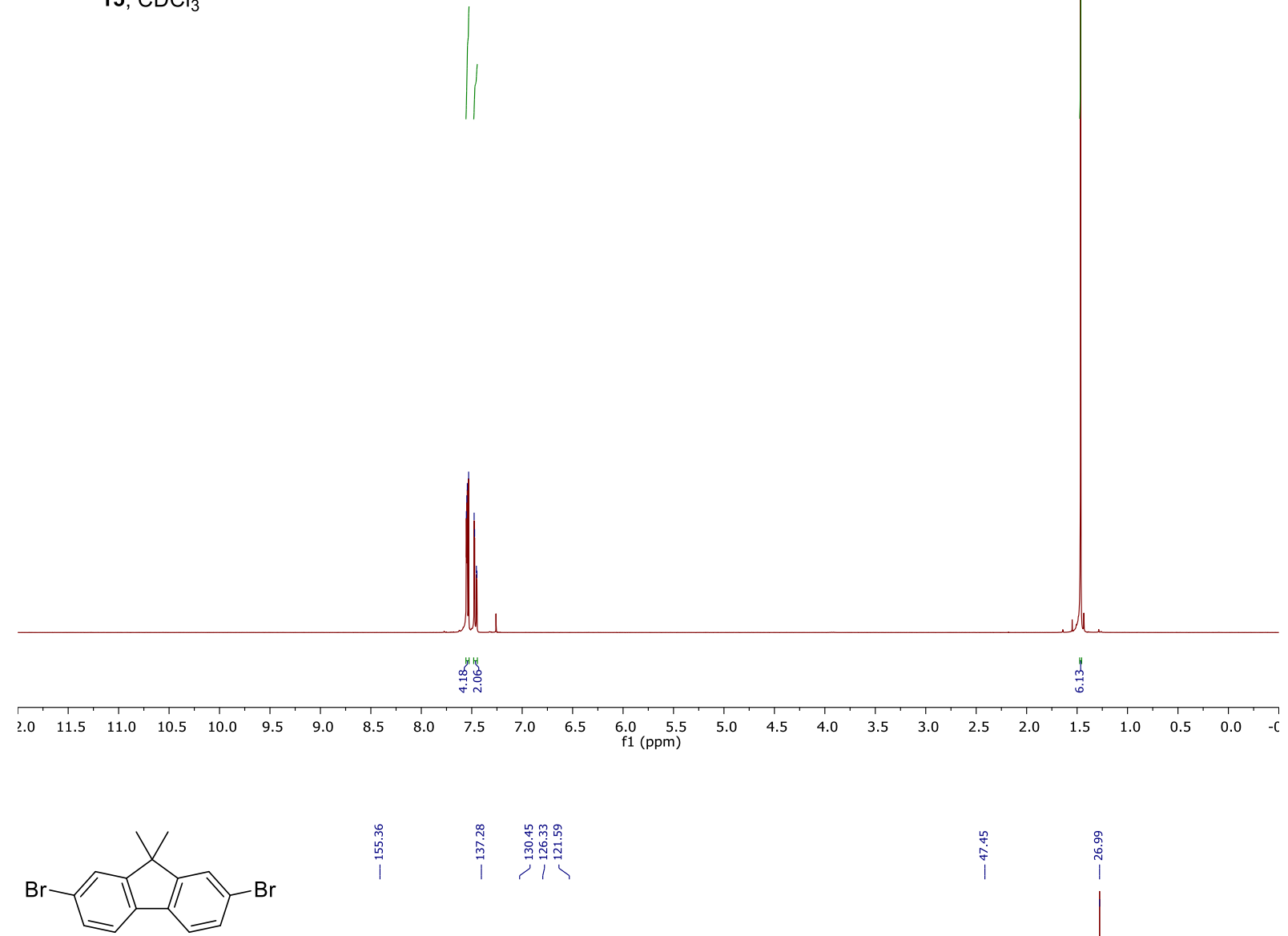

15, $\mathrm{CDCl}_{3}$

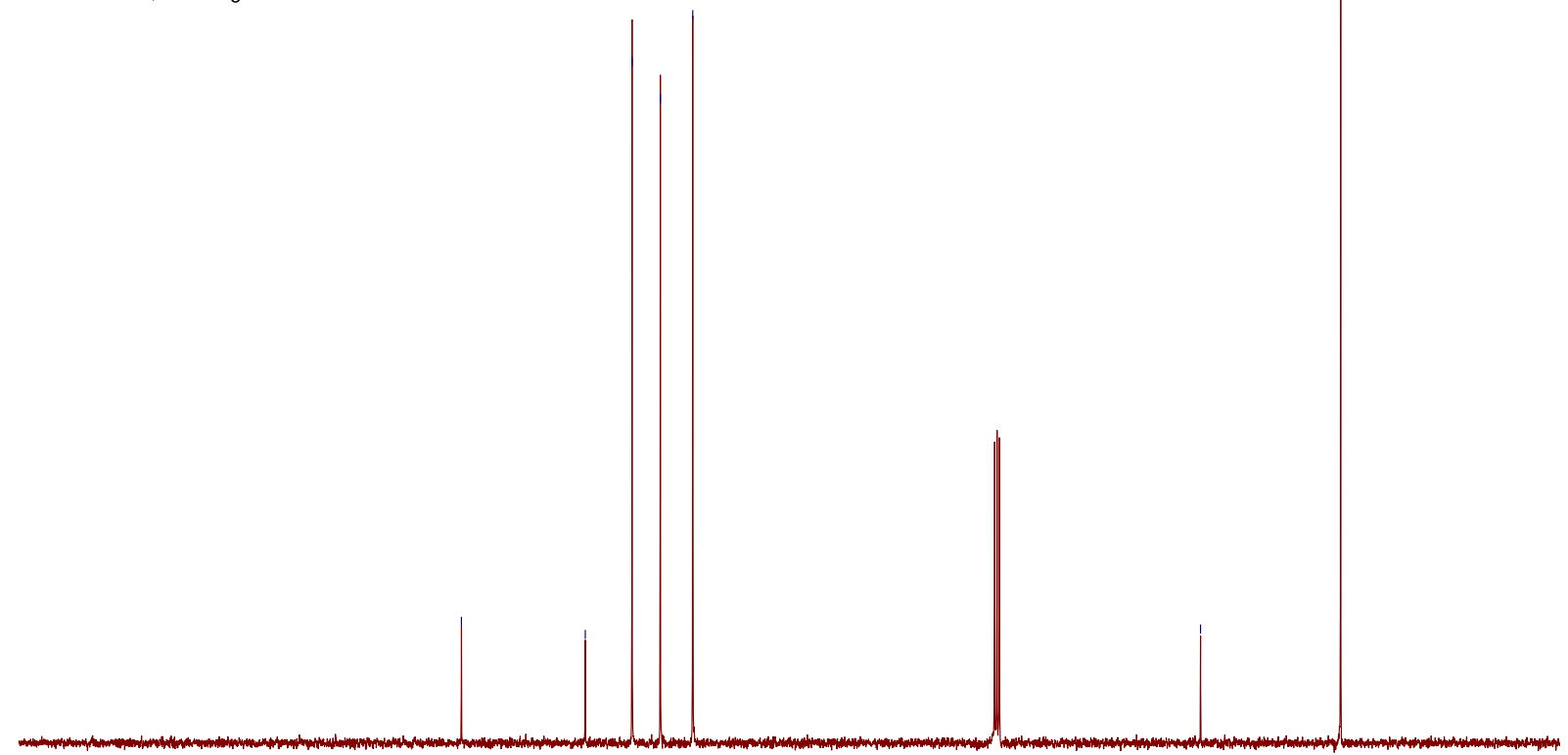

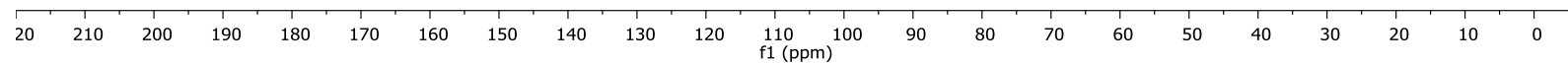




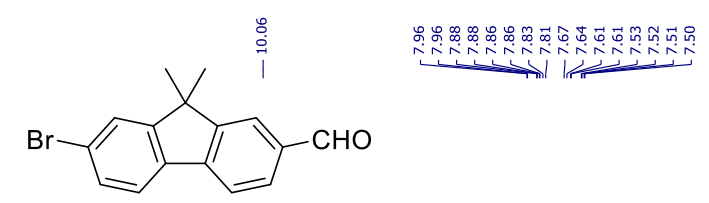

16, $\mathrm{CDCl}_{3}$
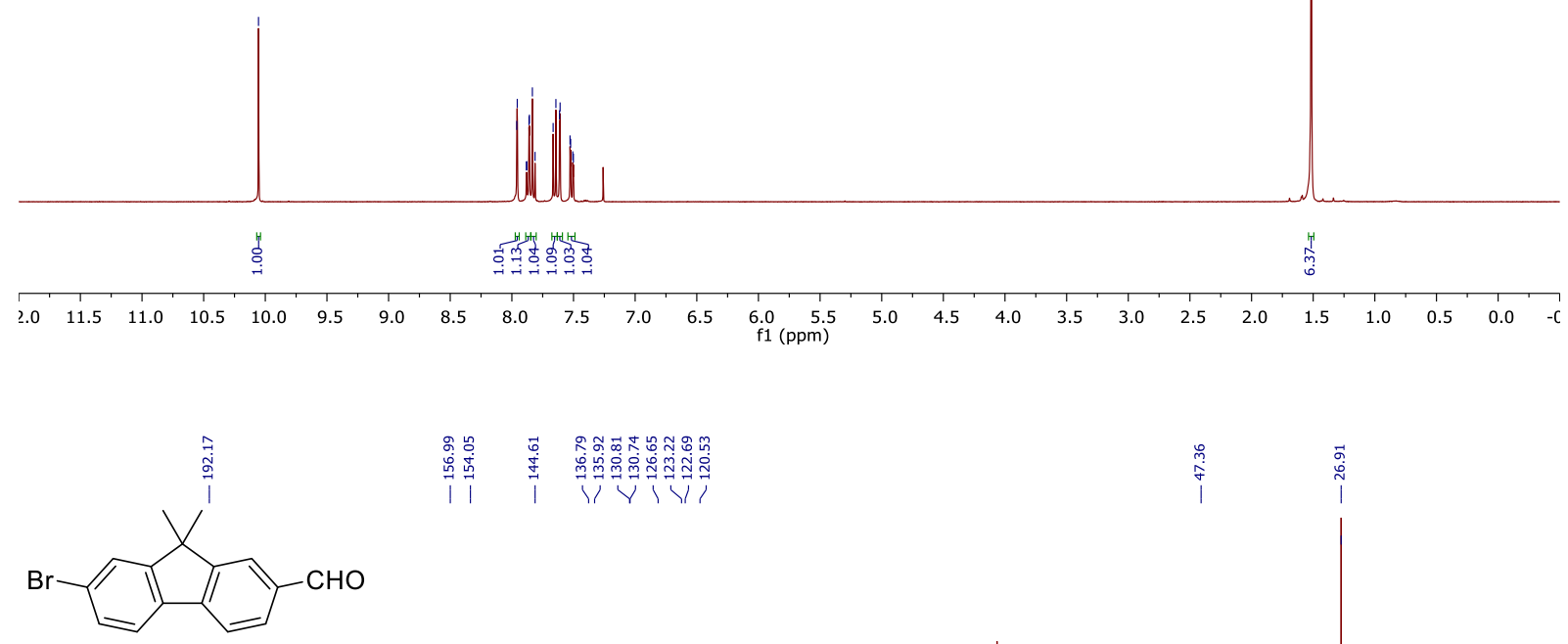

16, $\mathrm{CDCl}_{3}$

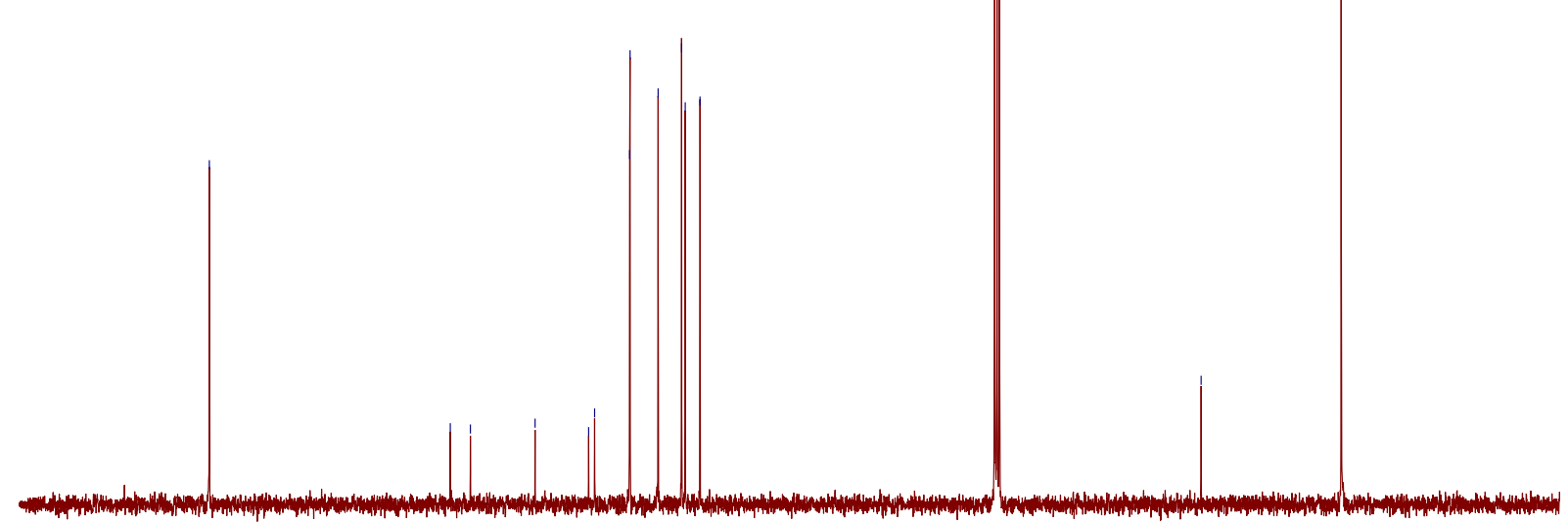

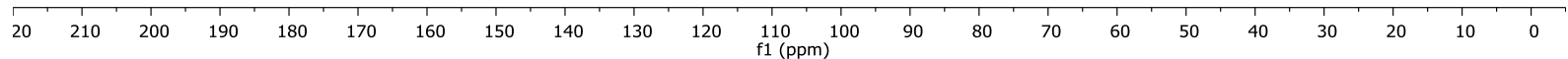



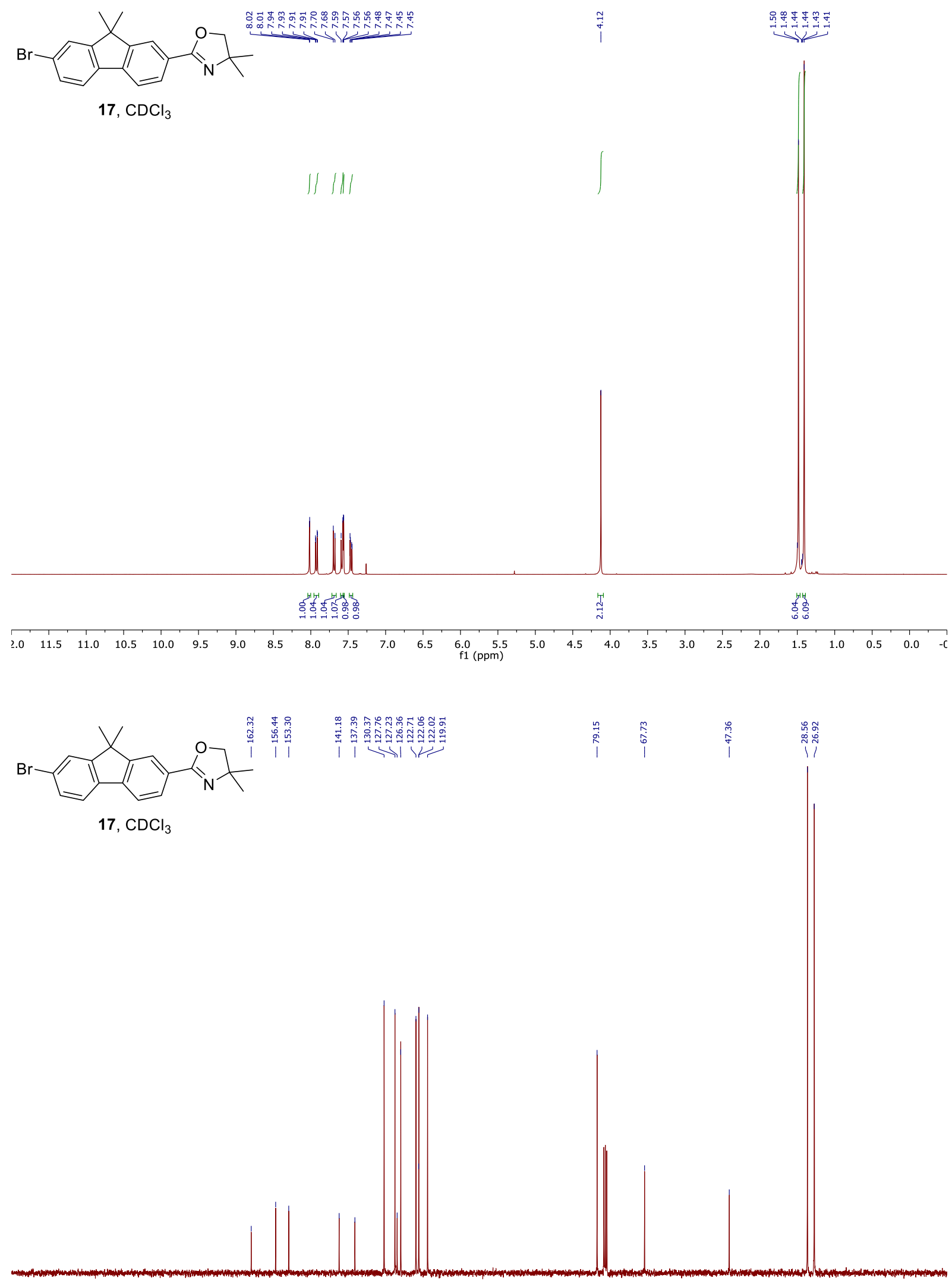

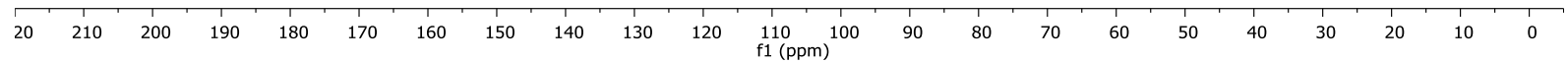



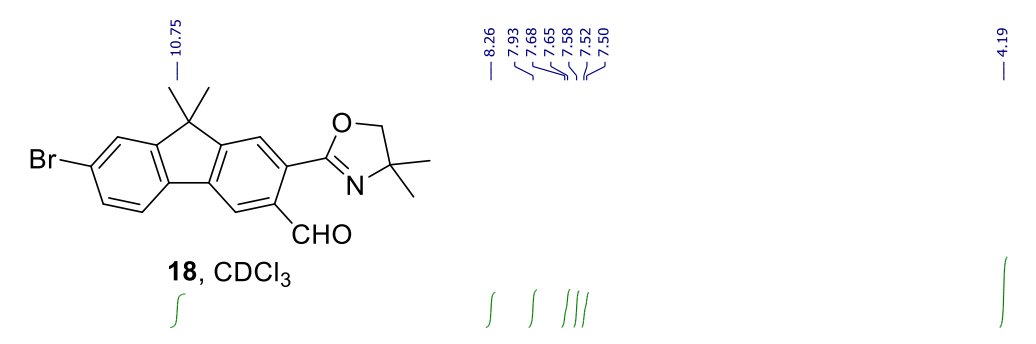

18, $\mathrm{CDCl}_{3}$
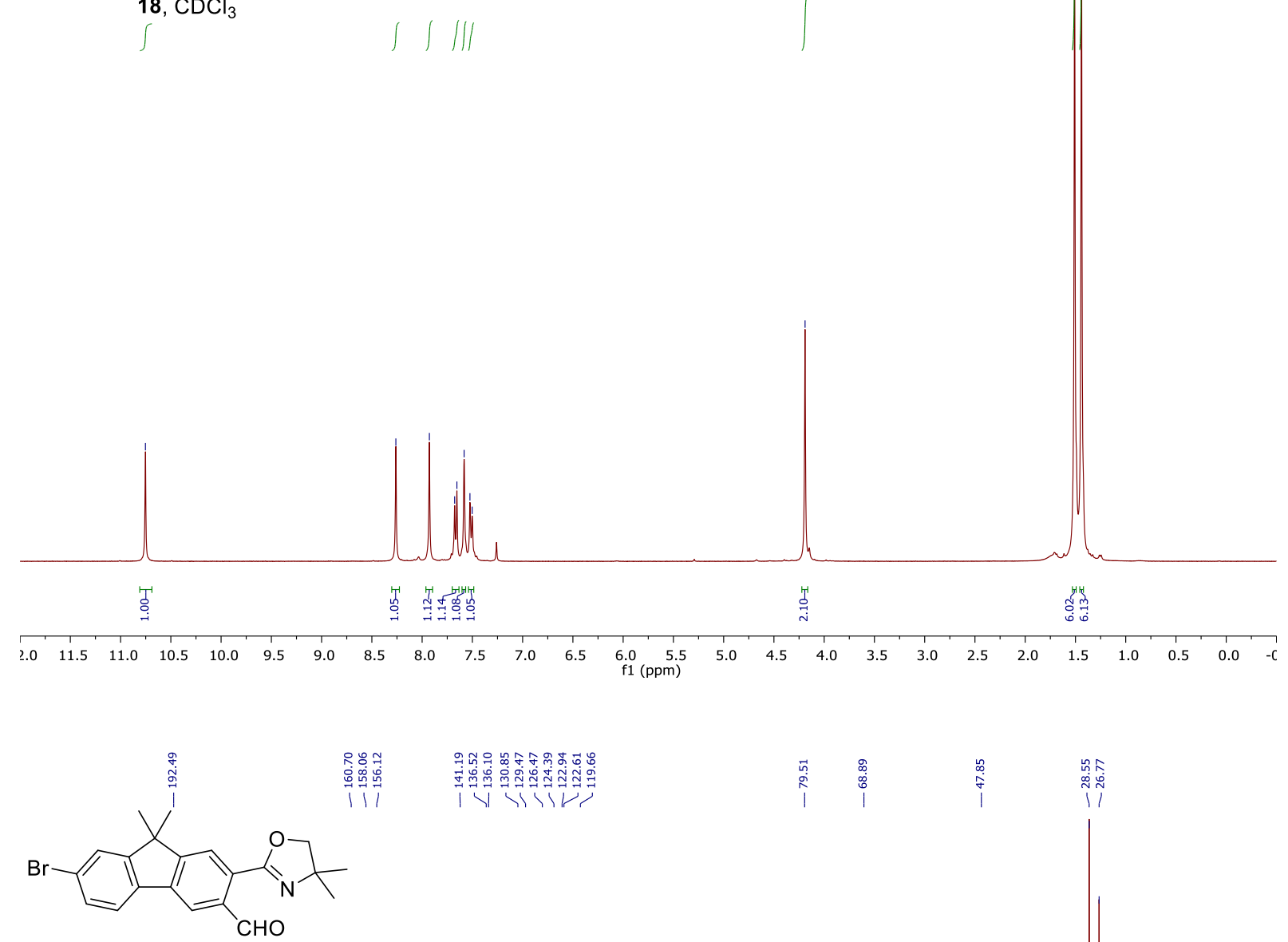

18, $\mathrm{CDCl}_{3}$

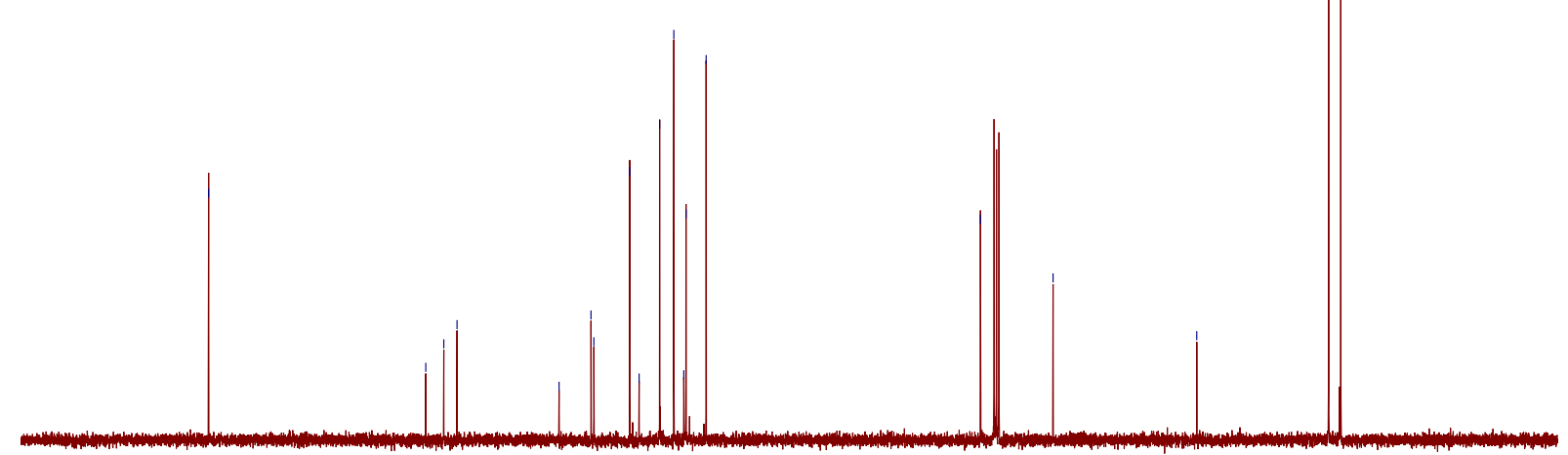

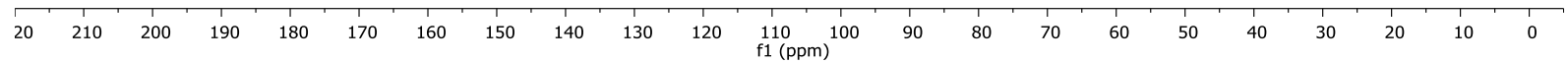



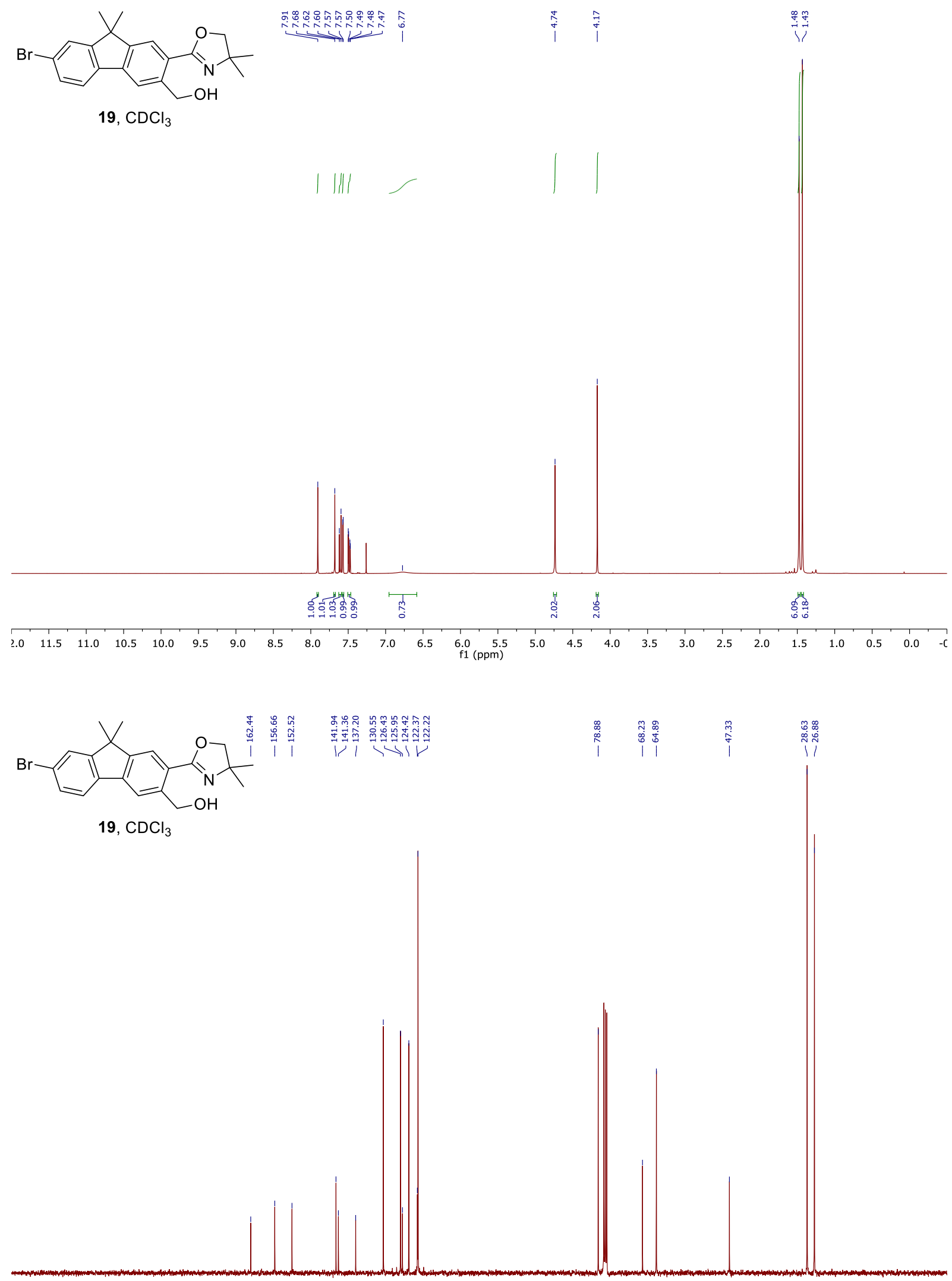

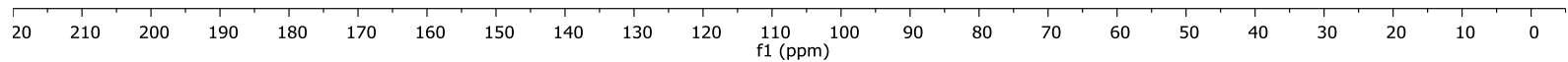



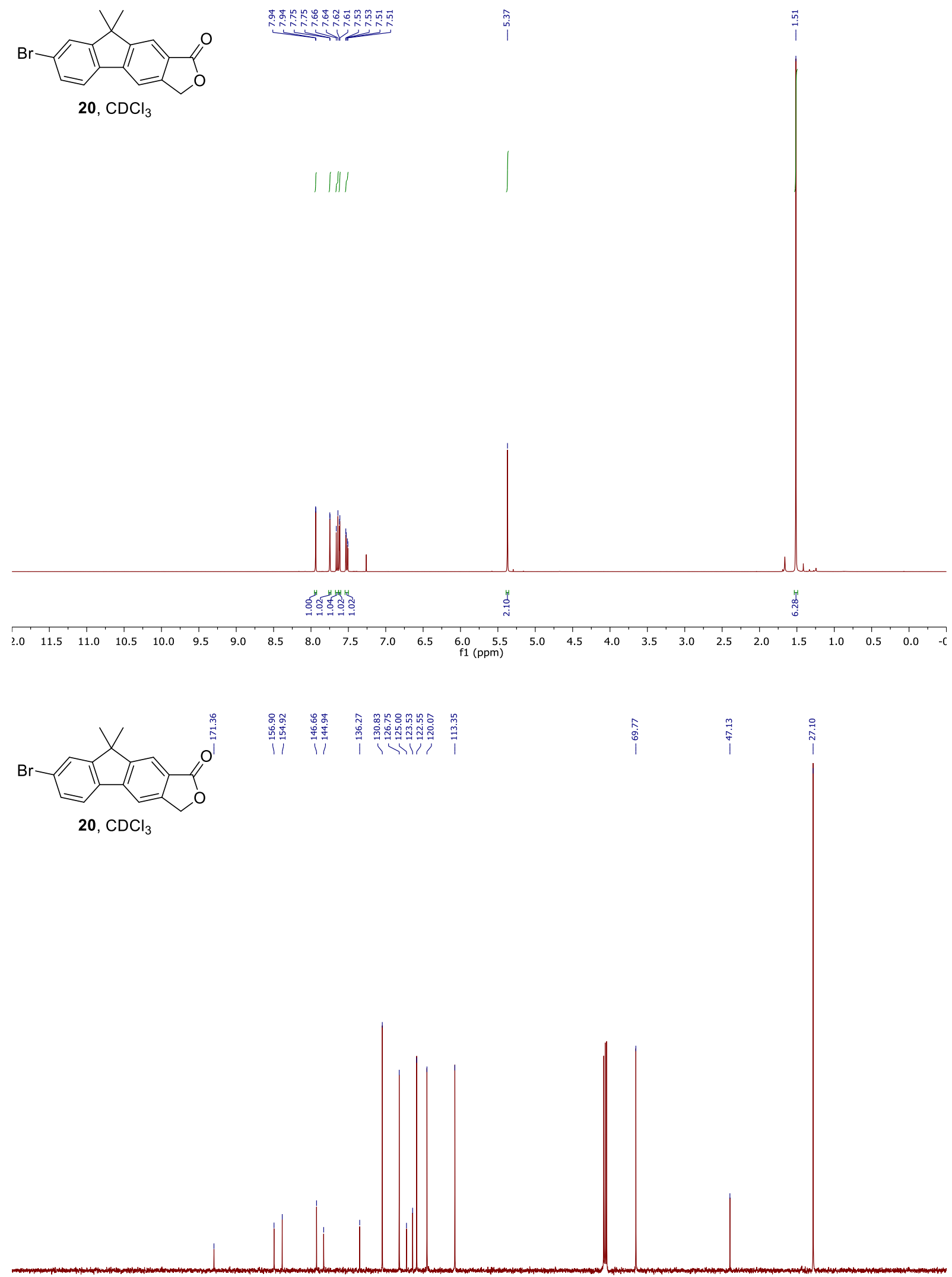

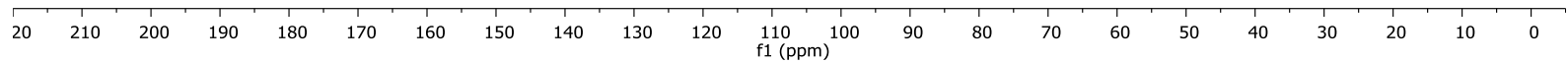



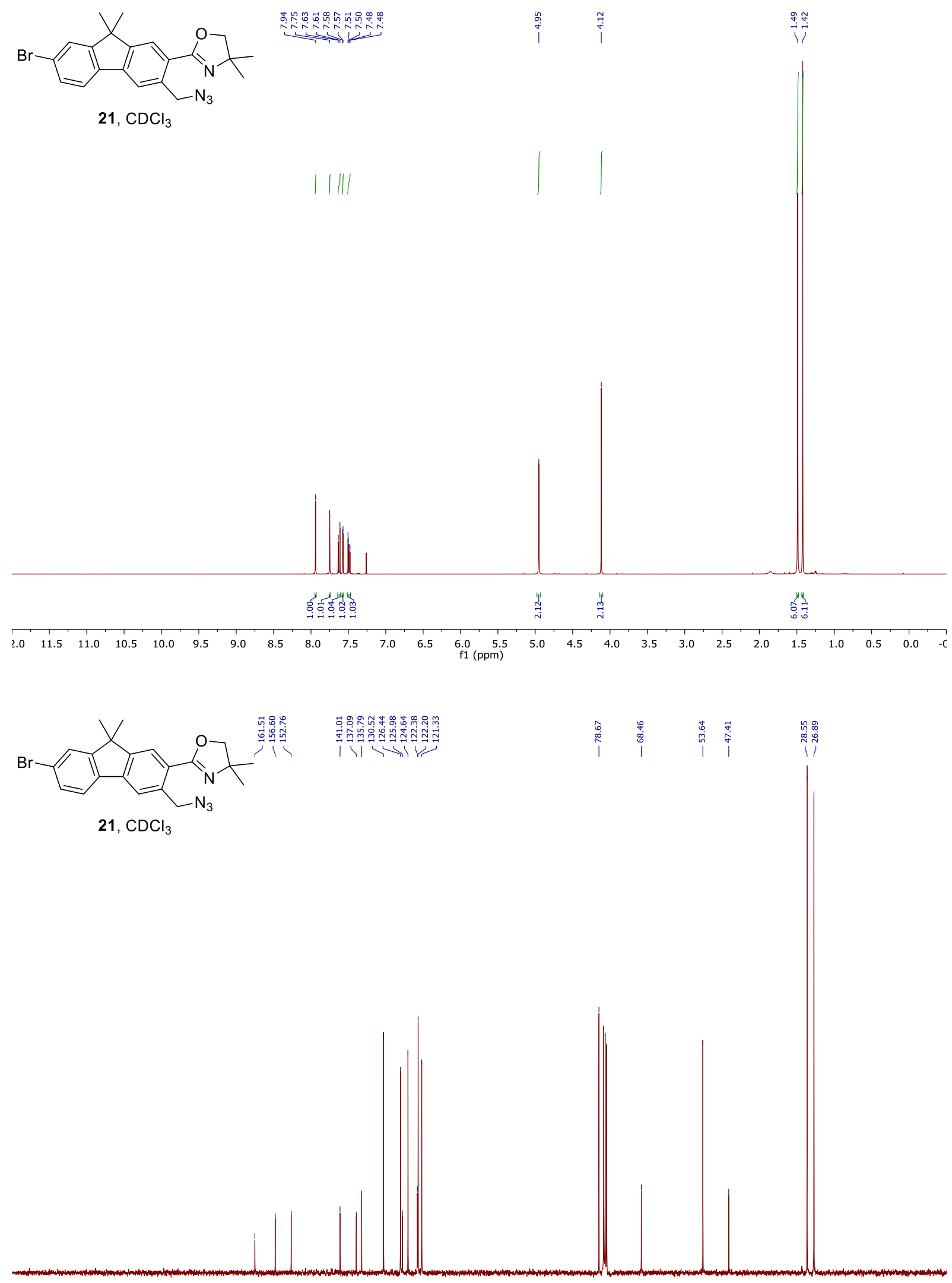

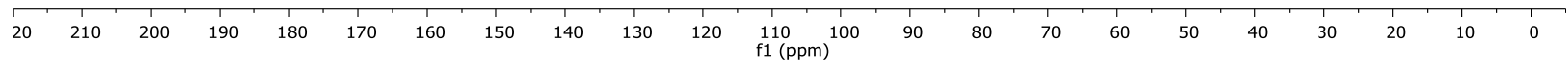




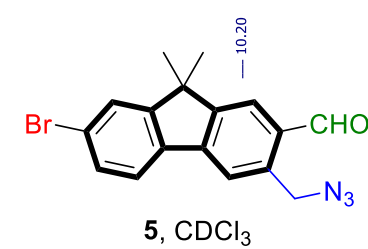

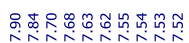

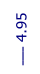

|I III
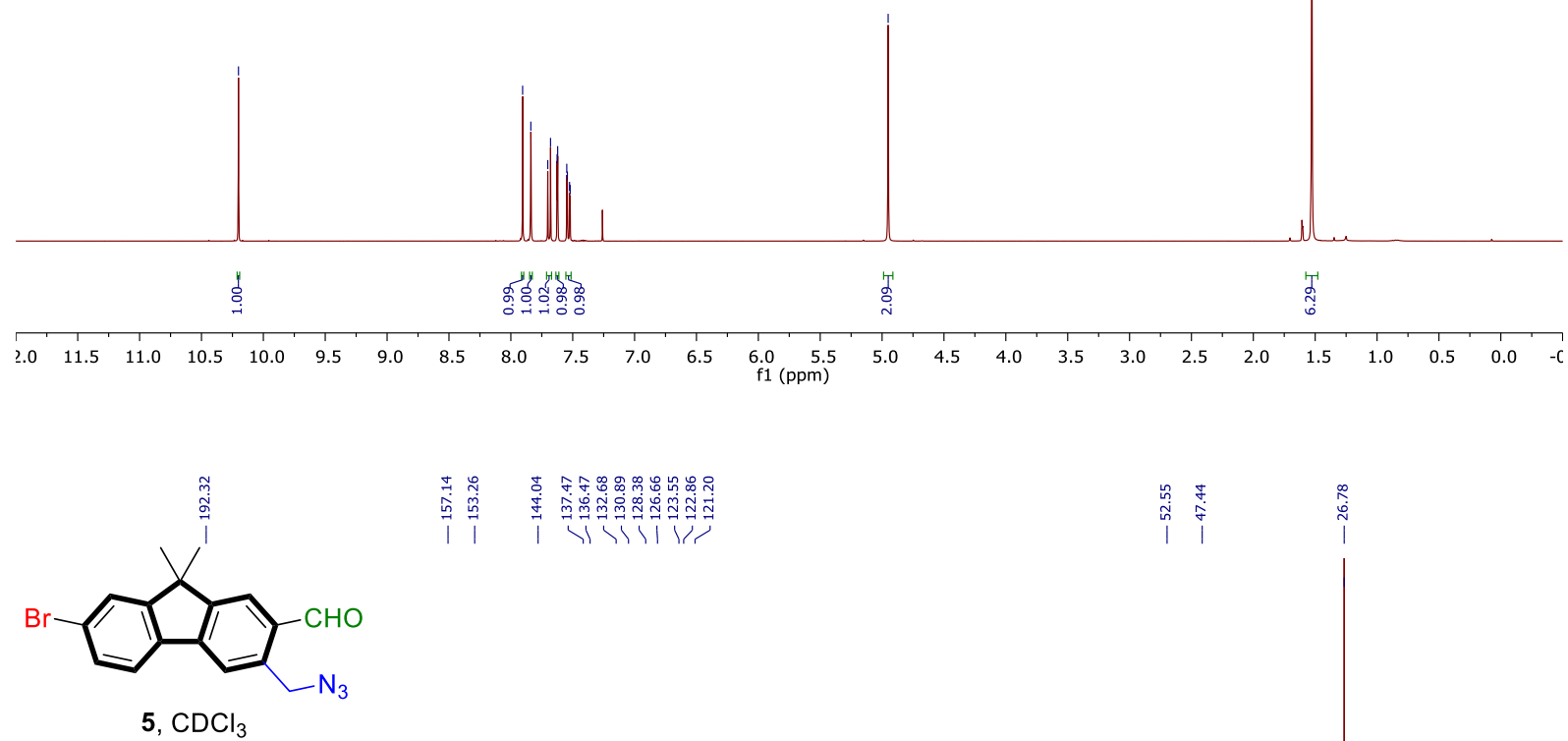

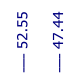

$\stackrel{\substack{0 \\ \stackrel{\infty}{i}}}{1}$

5, $\mathrm{CDCl}_{3}$

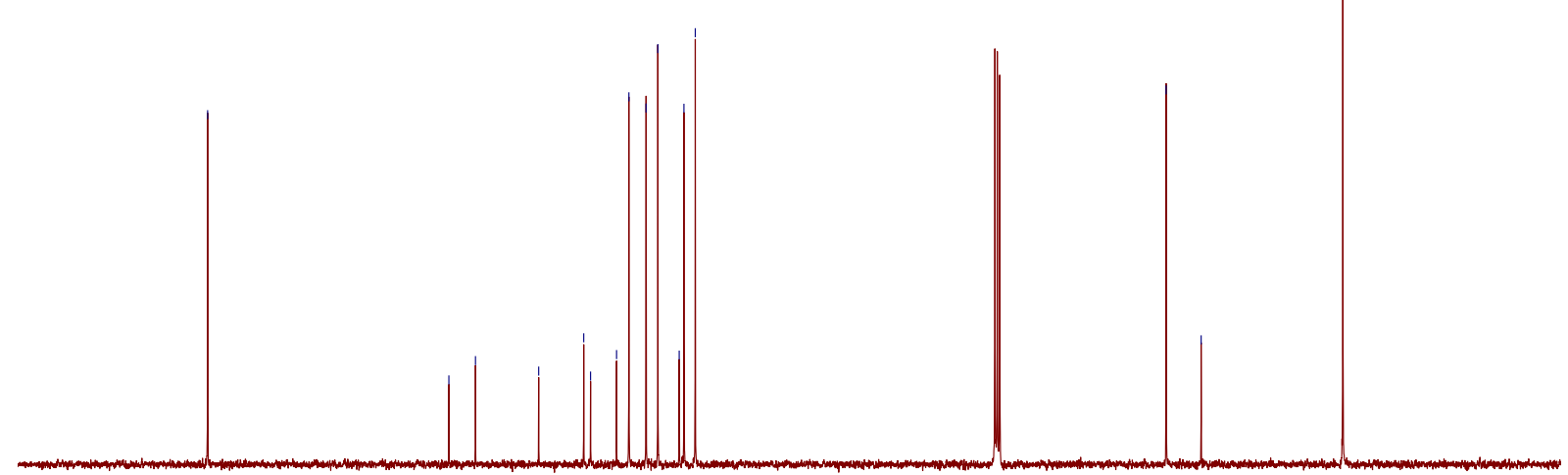

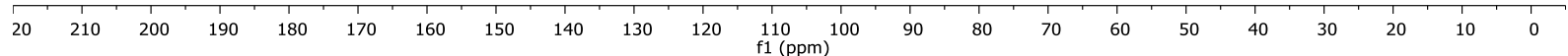



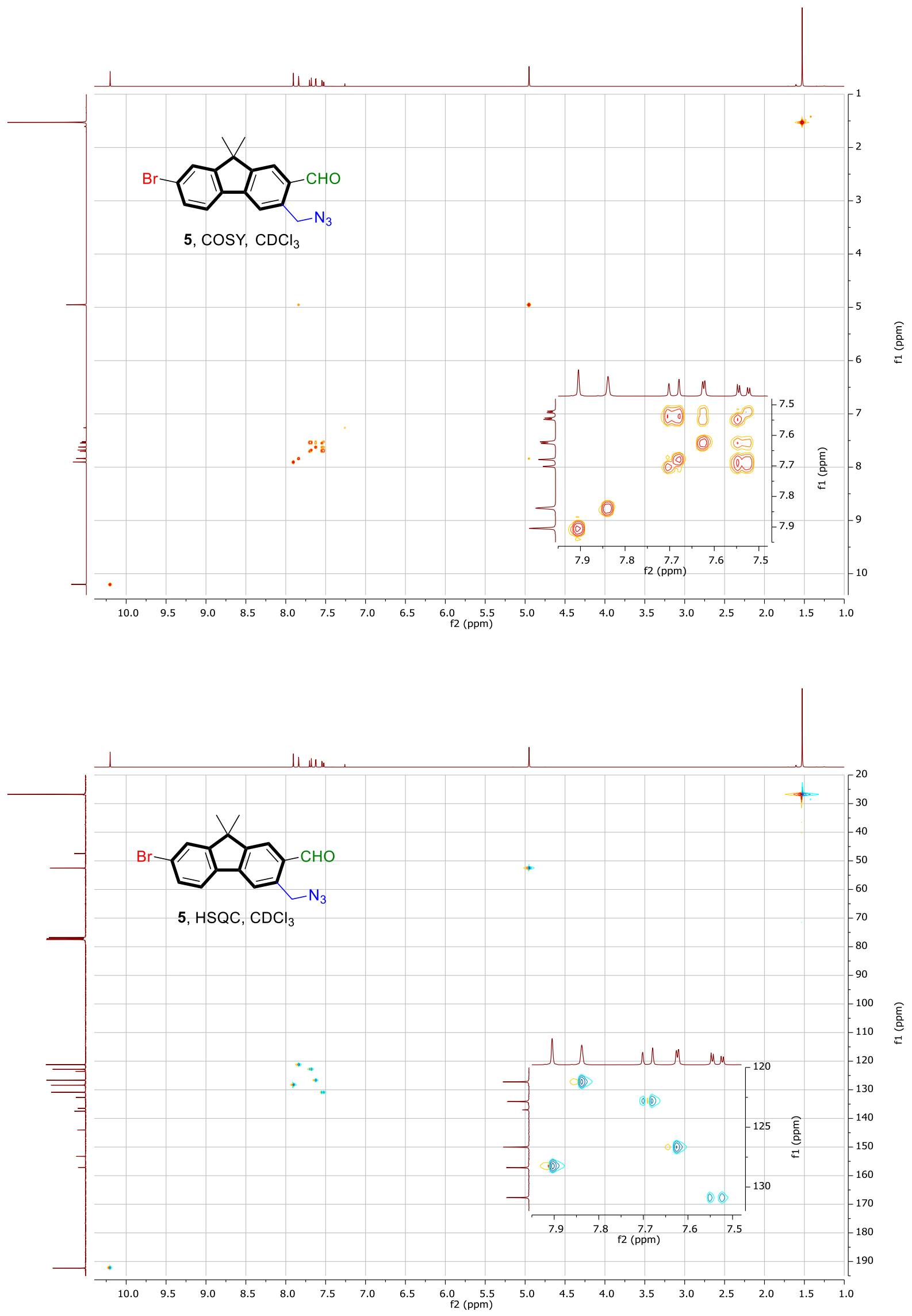


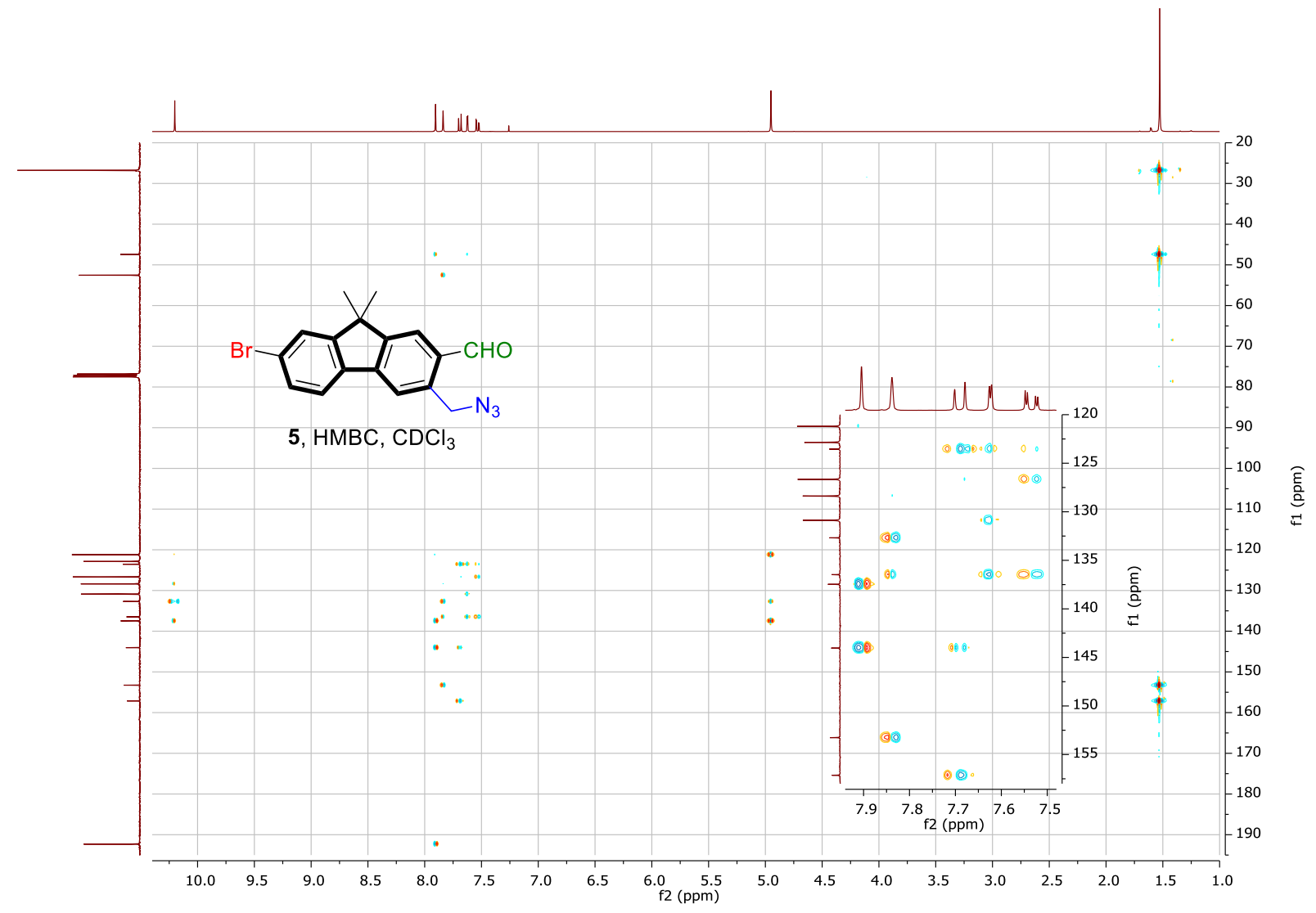




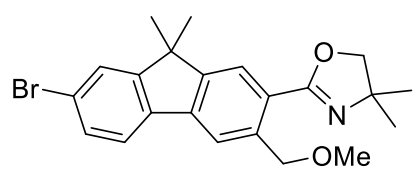

22, $\mathrm{CDCl}_{3}$

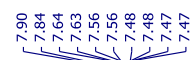

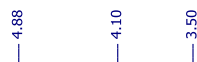

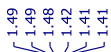

II III

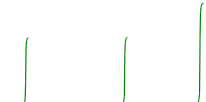

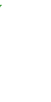



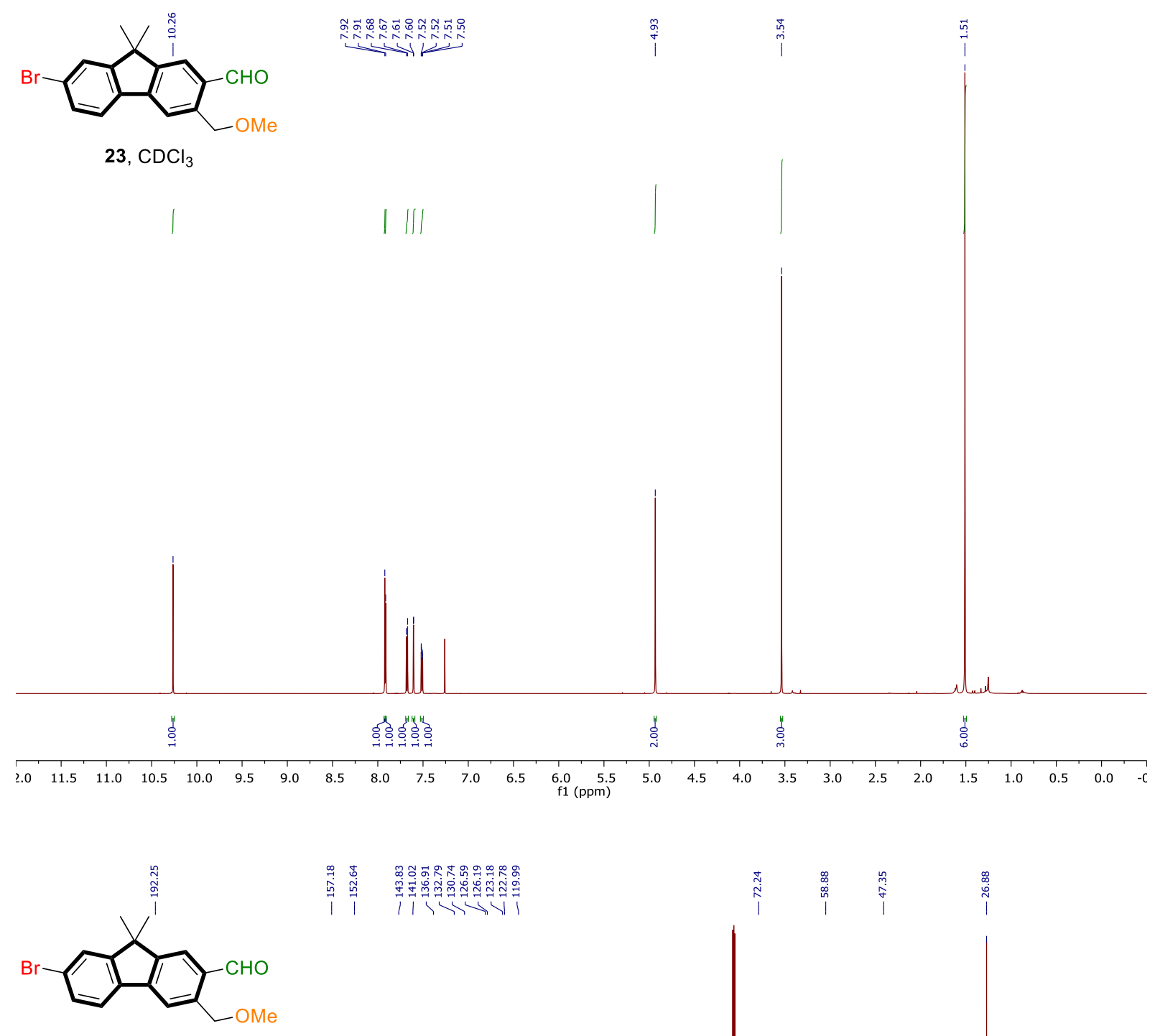

|

23, $\mathrm{CDCl}_{3}$

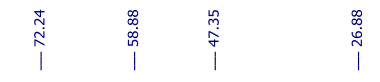




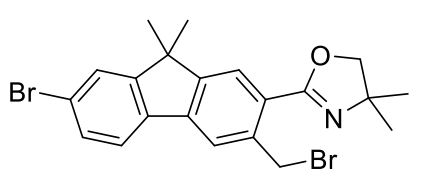

24, $\mathrm{CDCl}_{3}$

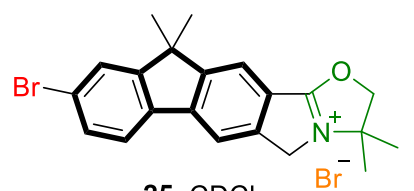

25, $\mathrm{CDCl}_{3}$

$\mathbf{2 4 : 2 5}=1.00: 0.43$
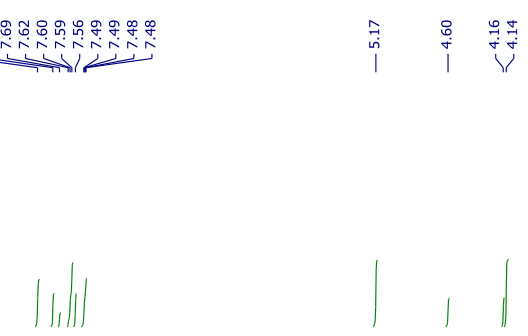

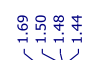

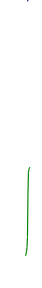

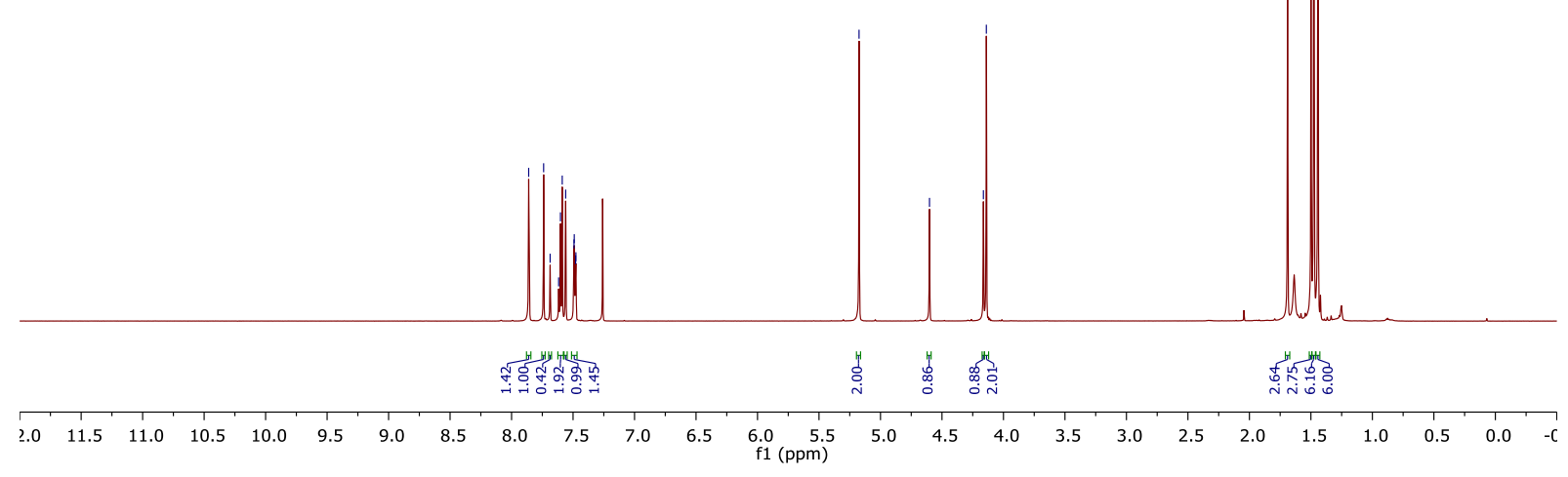

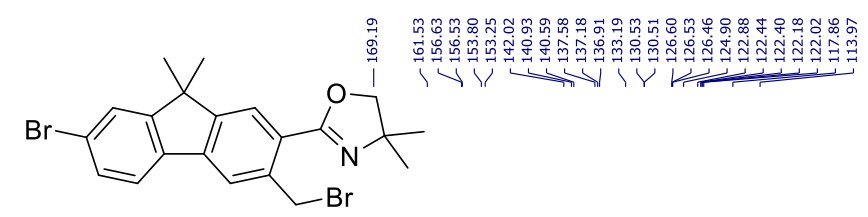

24, $\mathrm{CDCl}_{3}$

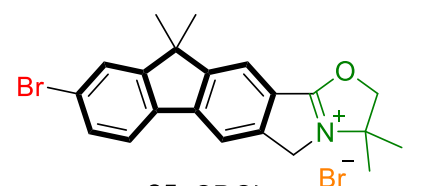

25, $\mathrm{CDCl}_{3}$

$\mathbf{2 4}: \mathbf{2 5}=1.00: 0.43$

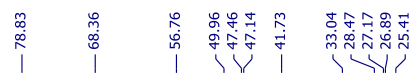




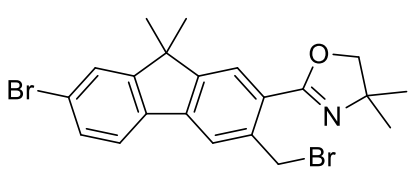

24, $\mathrm{CDCl}_{3}$

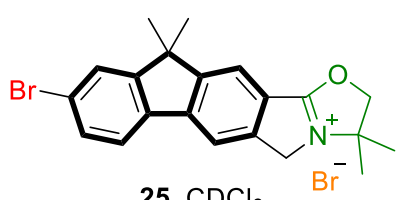

25, $\mathrm{CDCl}_{3}$

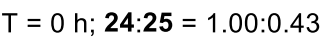

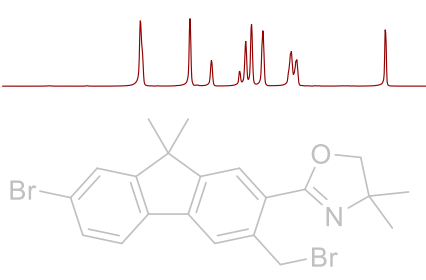

24, $\mathrm{CDCl}_{3}$

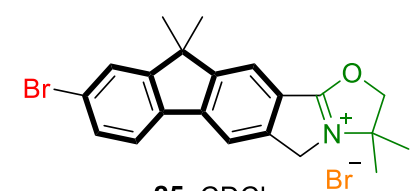

25, $\mathrm{CDCl}_{3}$

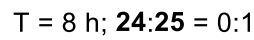

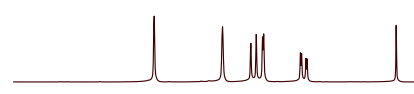

$\begin{array}{lllllllllllllllllllllllllllllllllllllllllllll}.2 & 8.1 & 8.0 & 7.9 & 7.8 & 7.7 & 7.6 & 7.5 & 7.4 & 7.3 & 7.2 & 7.1 & 5.3 & 5.2 & 5.1 & 5.0 & 4.9 & 4.8 & 4.7 & 4.6 & 4.5 & 4.4 & 4.3 & 4.2 & 4.1 & 4.0 & 3.9 & 1.9 & 1.8 & 1.7 & 1.6 & 1.5 & 1.4 & 1.3 & 1.2 & 1.1 & 1.0\end{array}$<smiles>CC1(C)COC(c2cc3c(cc2CBr)-c2ccc(Br)cc2C3(C)C)=N1</smiles>

24, $\mathrm{CDCl}_{3}$

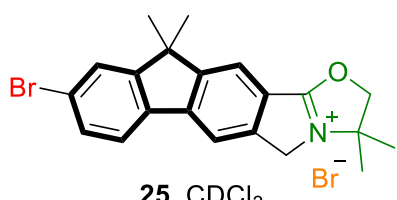

25, $\mathrm{CDCl}_{3}$

$\mathrm{T}=0 \mathrm{~h} ; \mathbf{2 4 : 2 5}=1.00: 0.43$

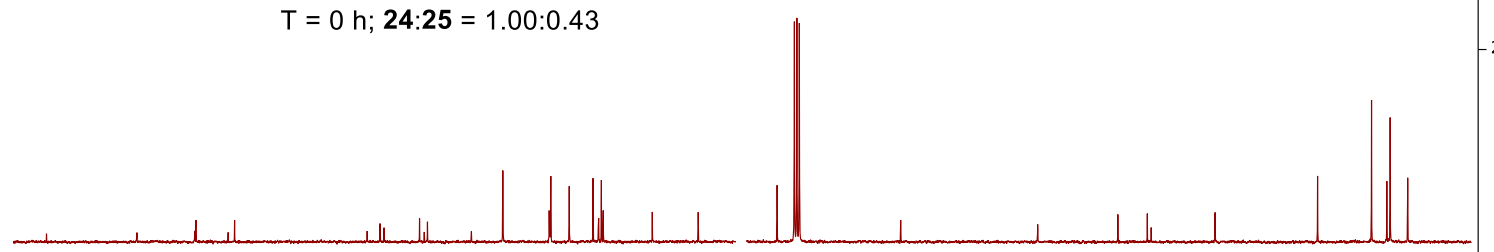

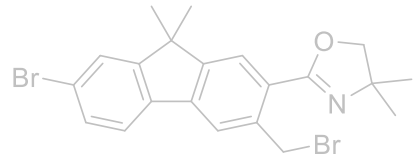

24, $\mathrm{CDCl}_{3}$

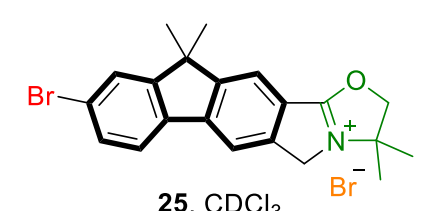

25, $\mathrm{CDCl}_{3}$

$T=8 \mathrm{~h} ; \mathbf{2 4 : 2 5}=0: 1$

$\begin{array}{llllllllllll}170 & 165 & 160 & 155 & 150 & 145 & 140 & 135 & 130 & 125 & 120 & 115 \\ \mathrm{f} 1(\mathrm{ppm})\end{array}$ 

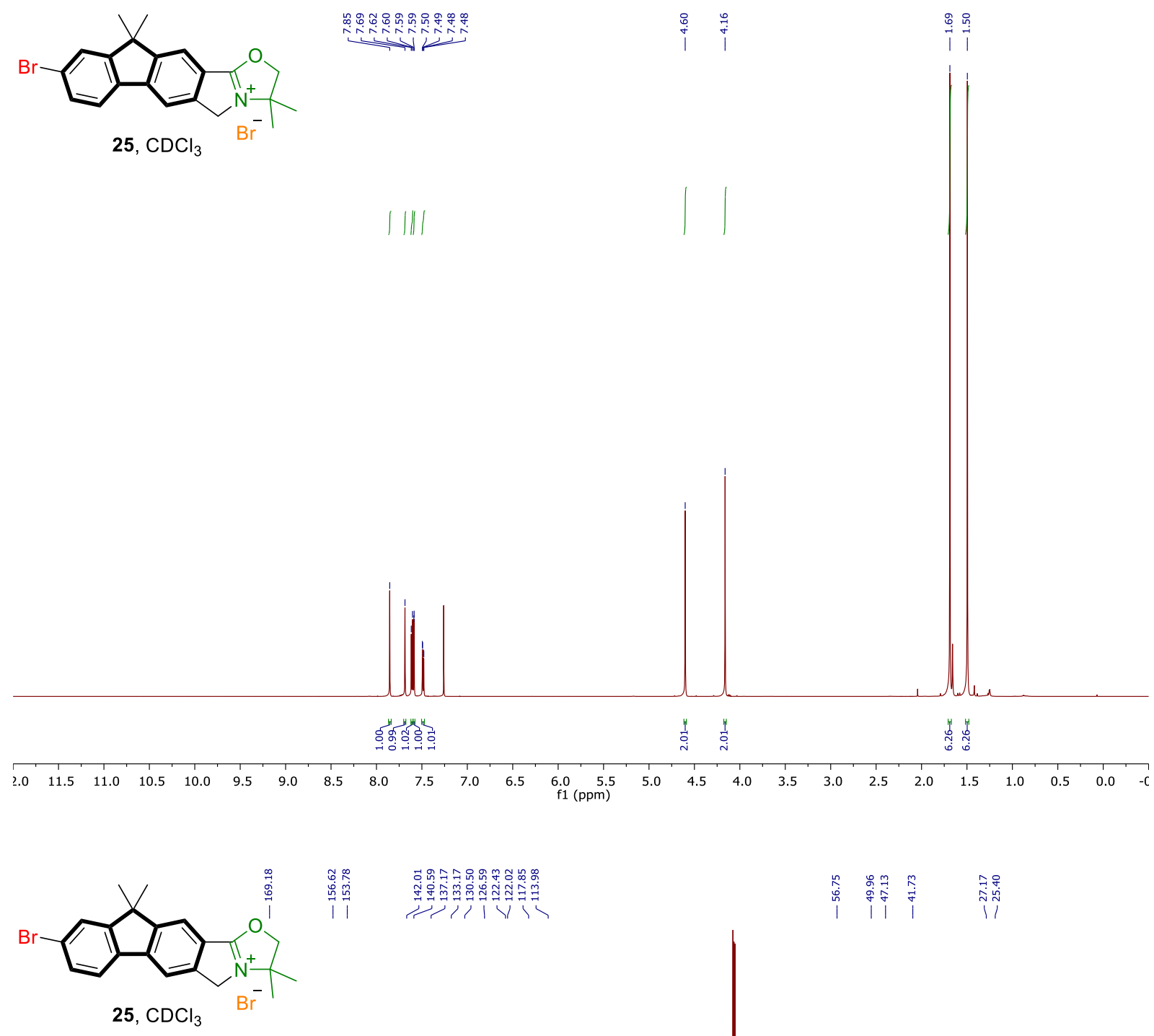

25, $\mathrm{CDCl}$ 

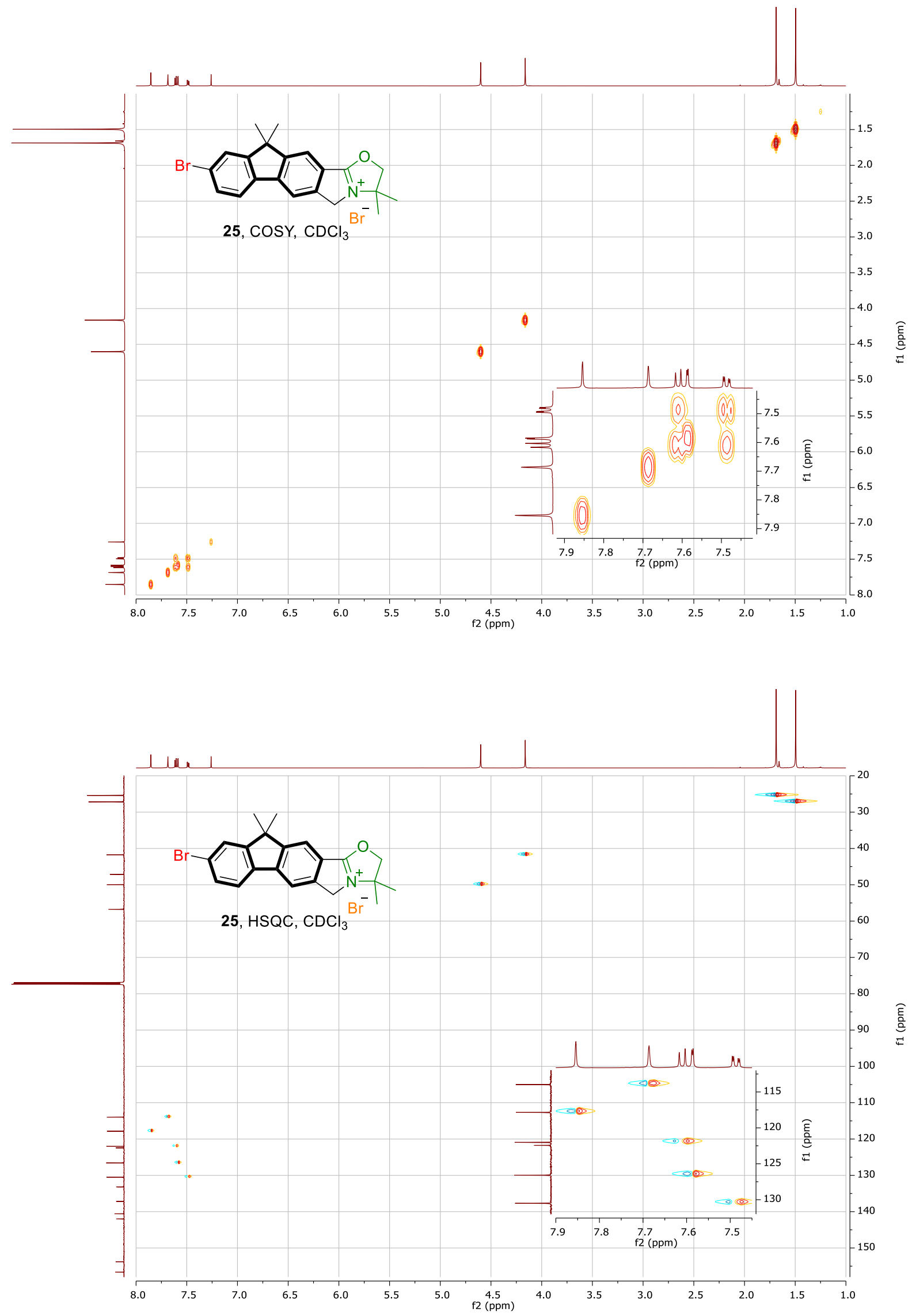

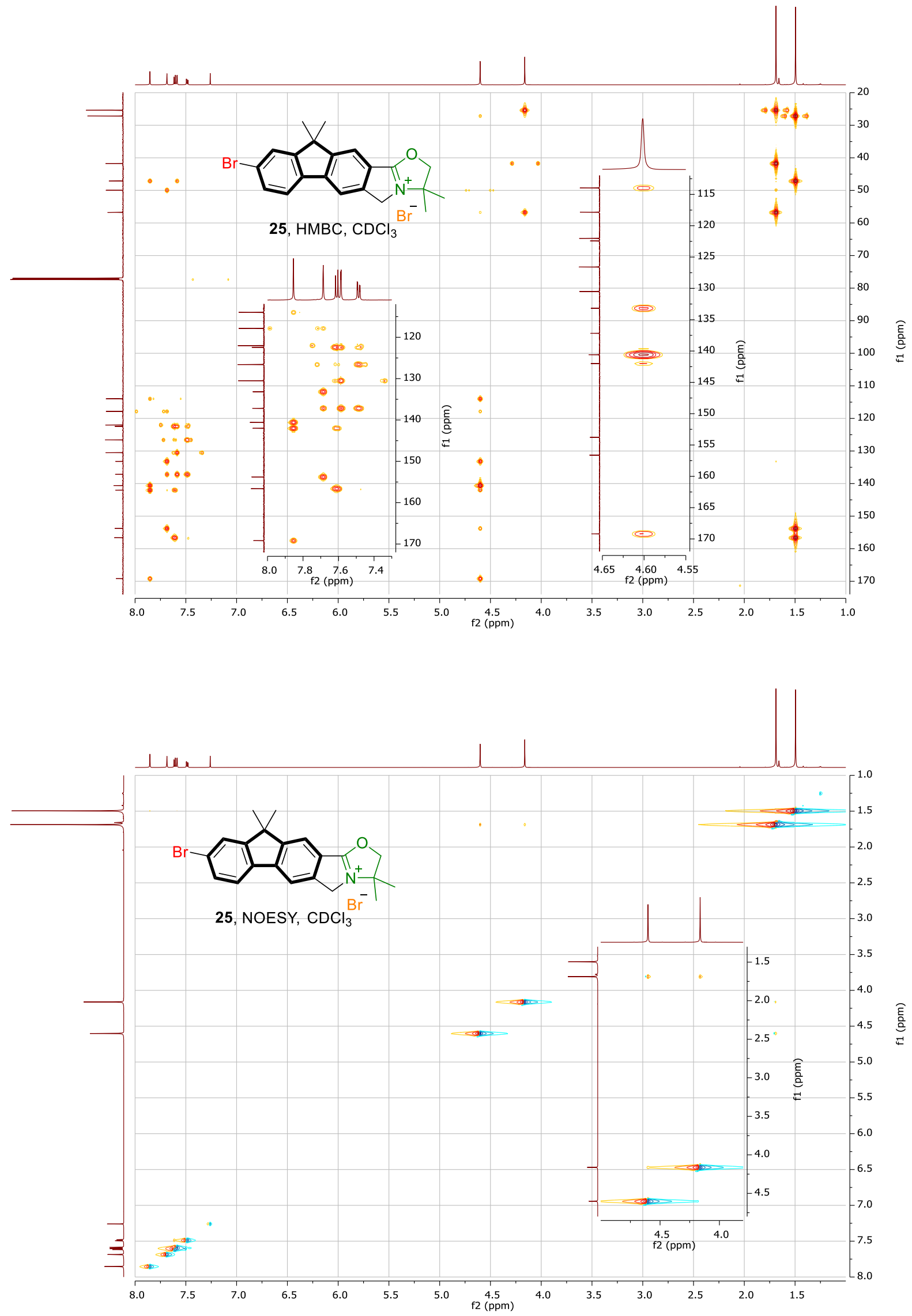

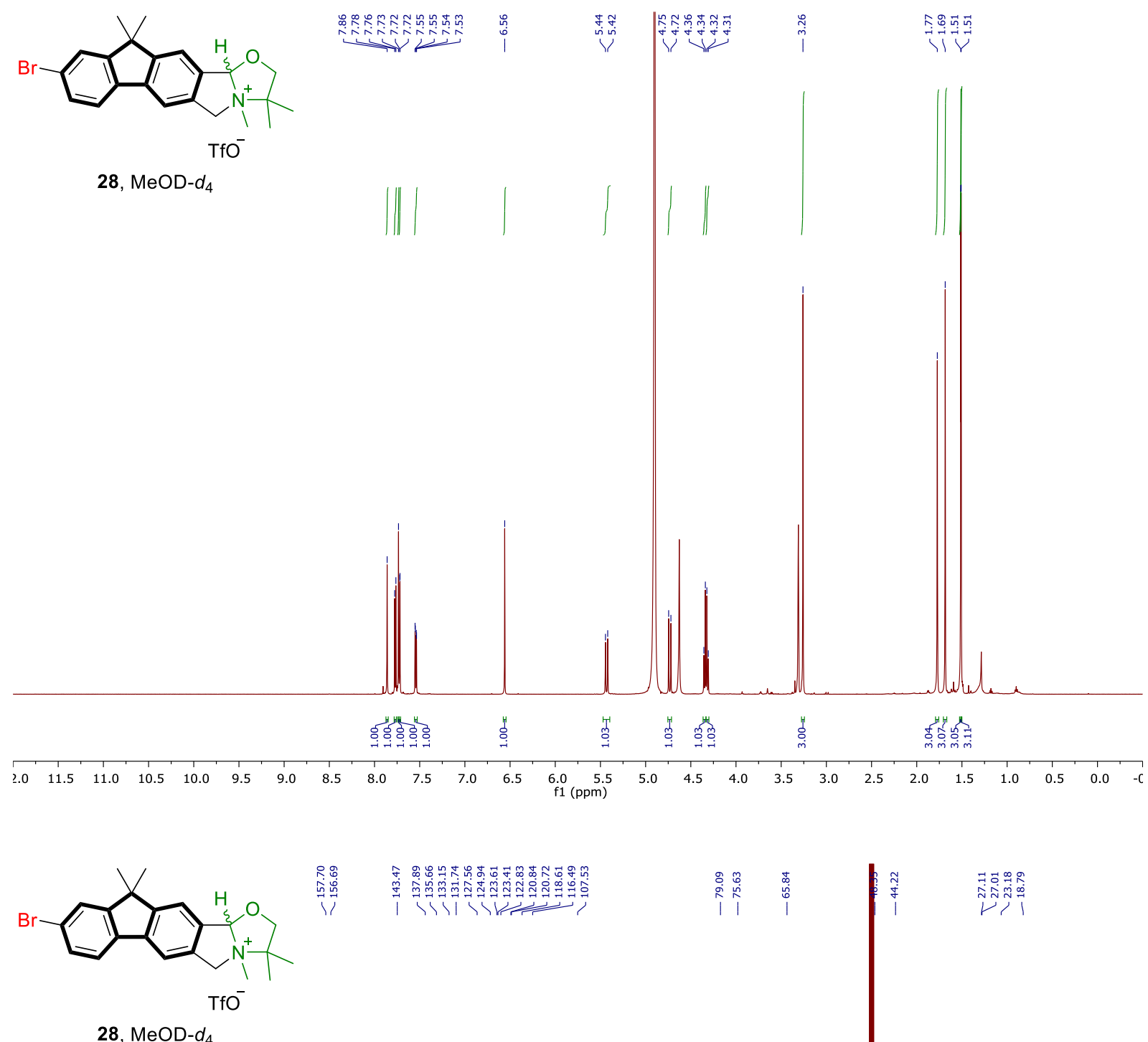

28, MeOD- $d_{4}$

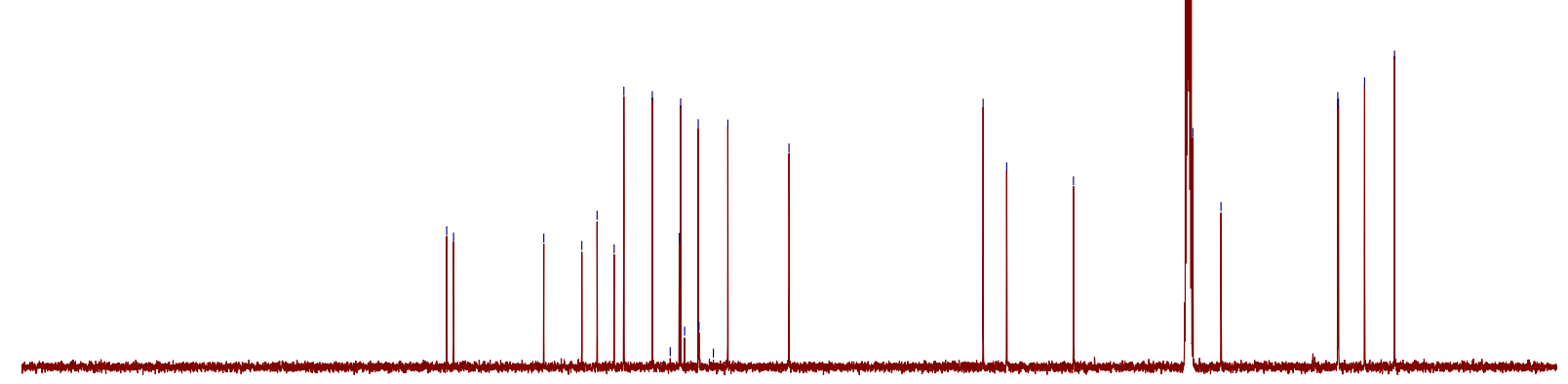

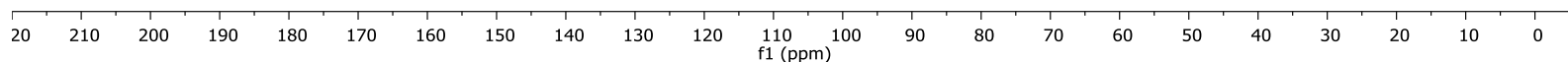




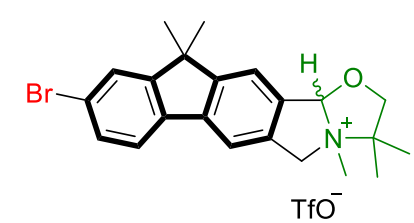

28, MeOD- $d_{4}$
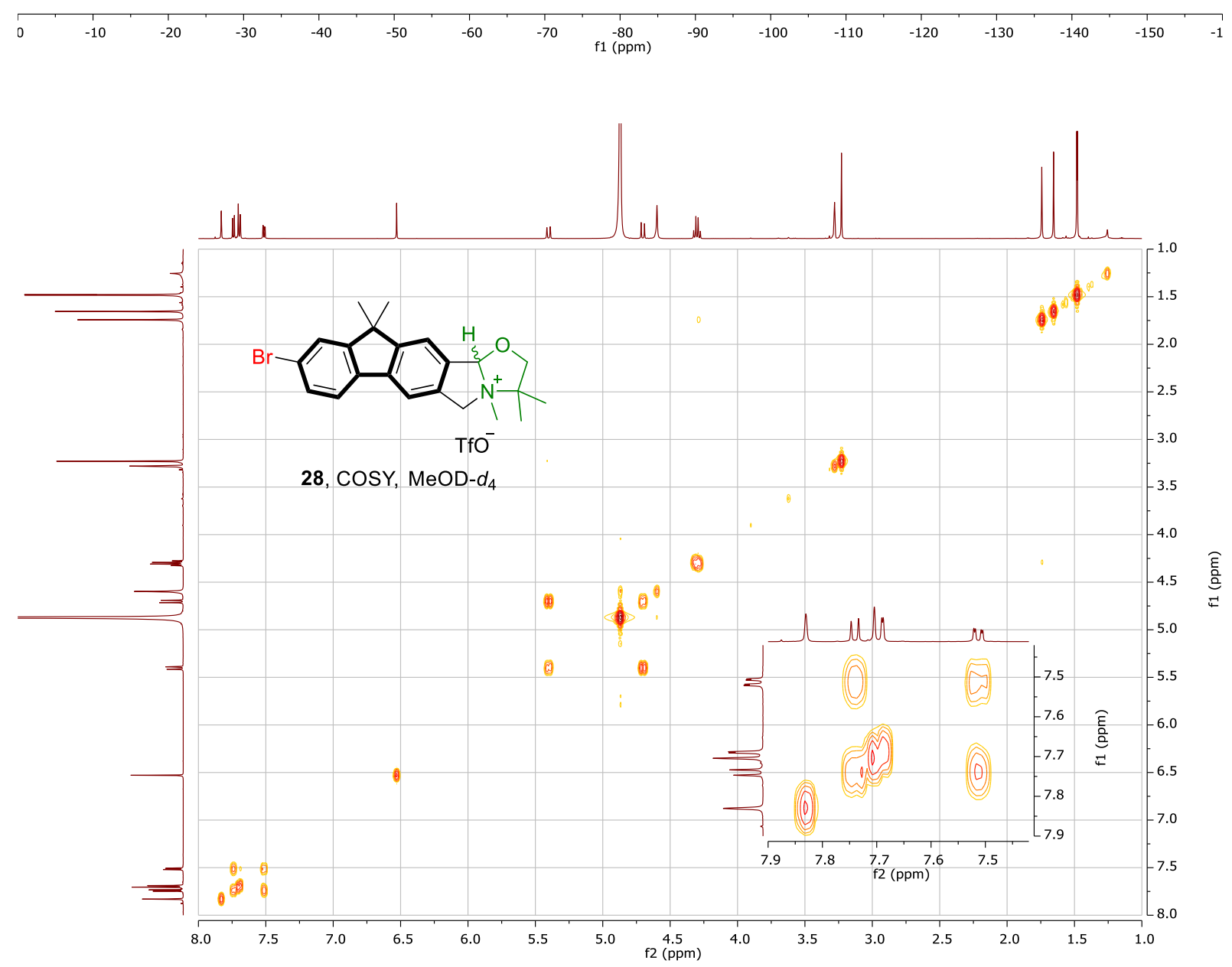

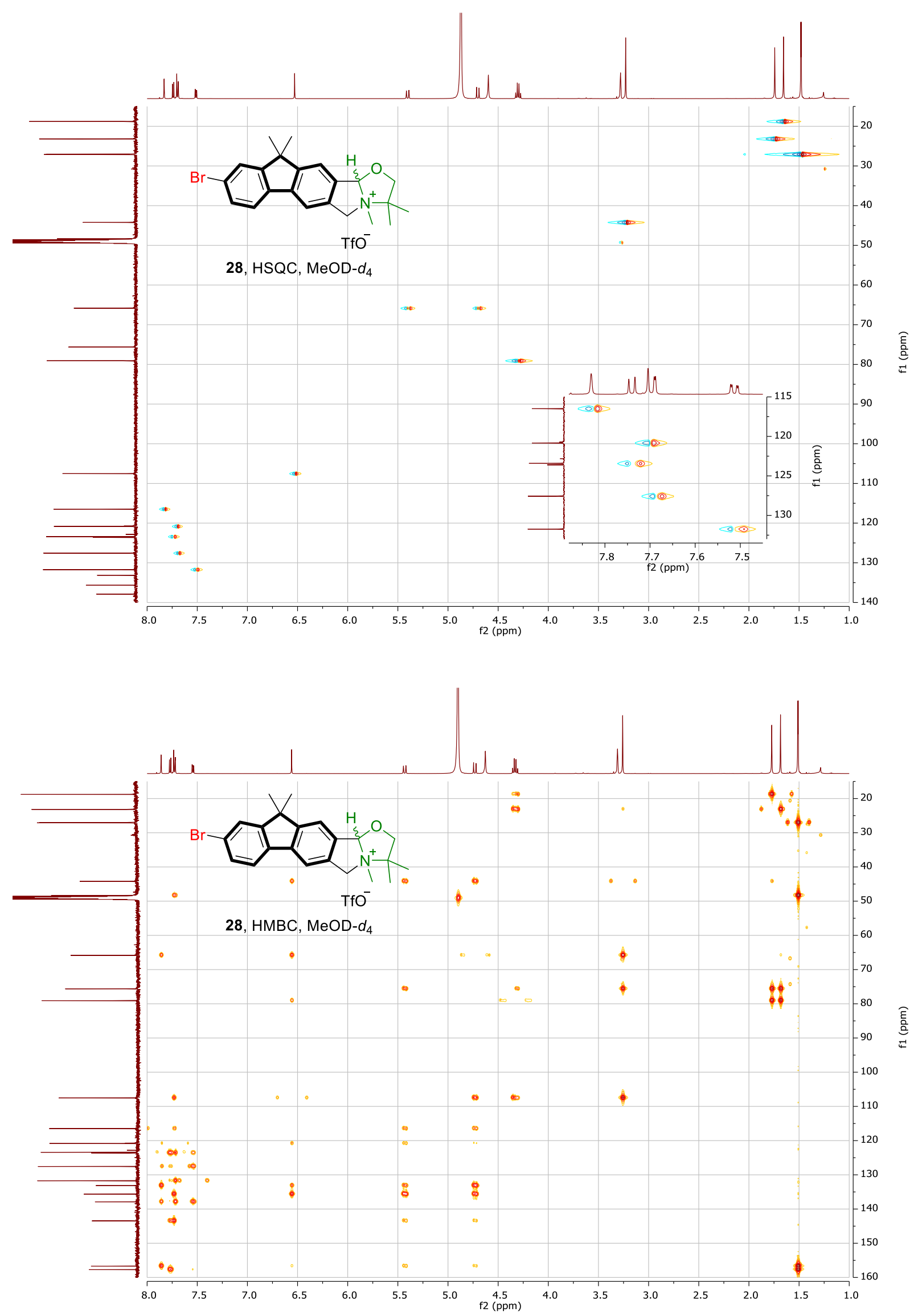


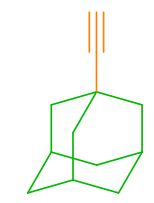

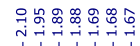

31, $\mathrm{CDCl}_{3}$
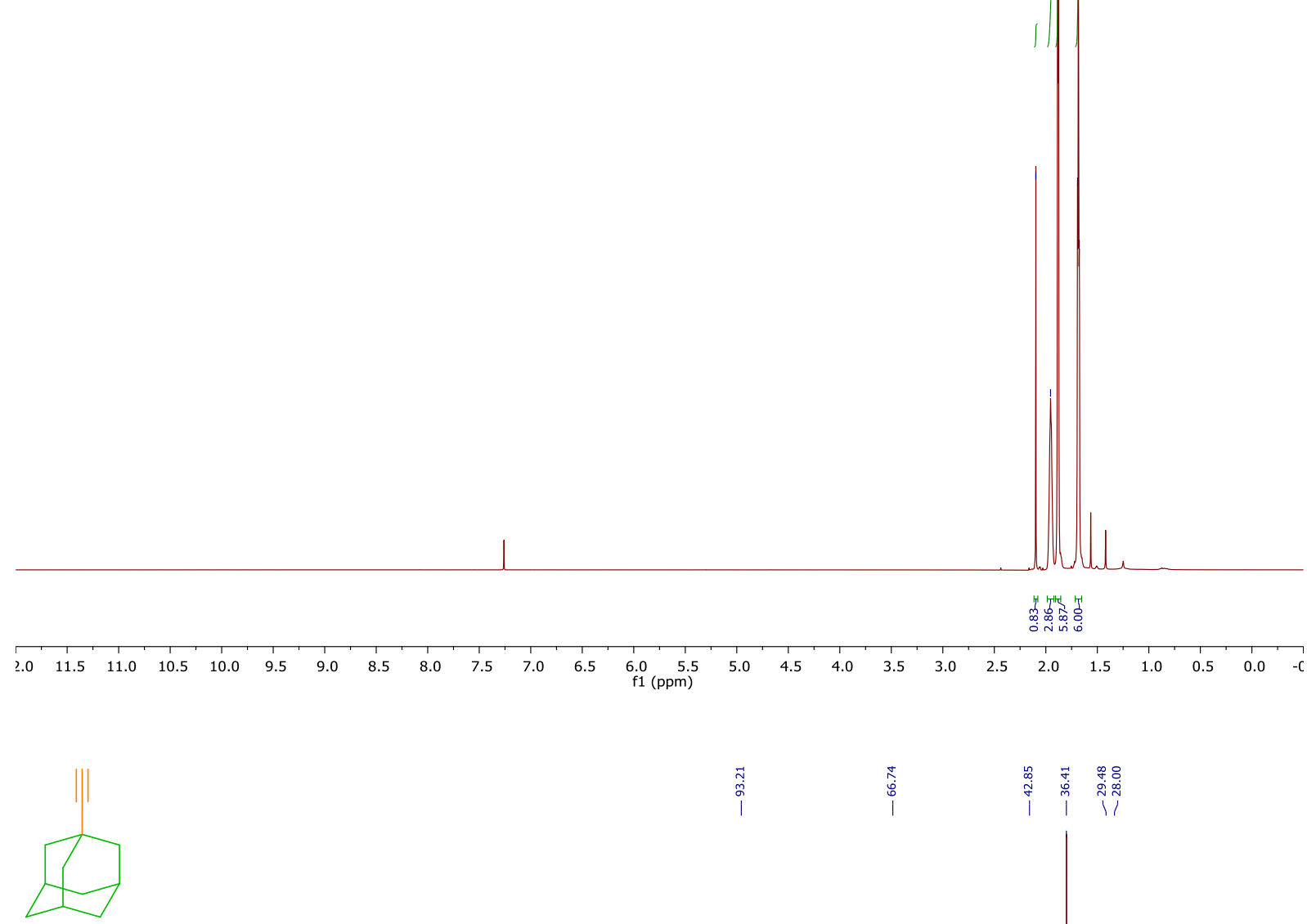

$\underset{\substack{n \\ \text { I }}}{\vec{N}}$

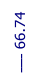

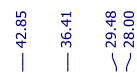

31, $\mathrm{CDCl}_{3}$

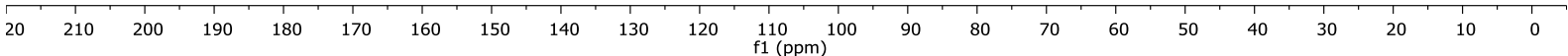



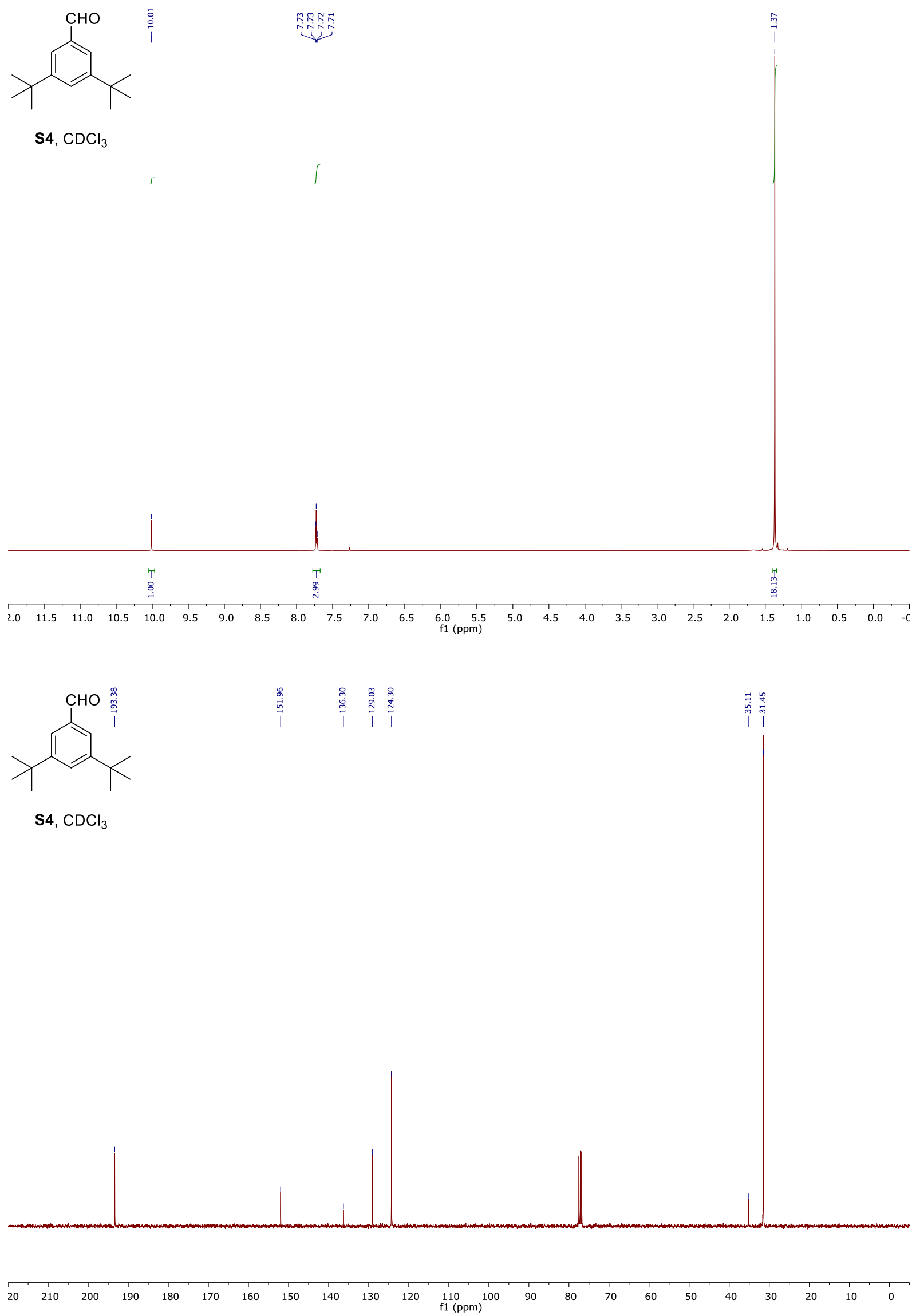

S112 


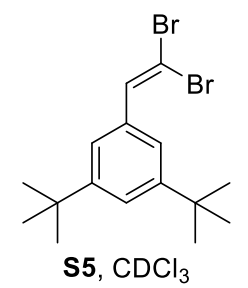

$\sqrt{\sqrt{n}}$

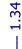

$\mathrm{S5}, \mathrm{CDCl}_{3}$

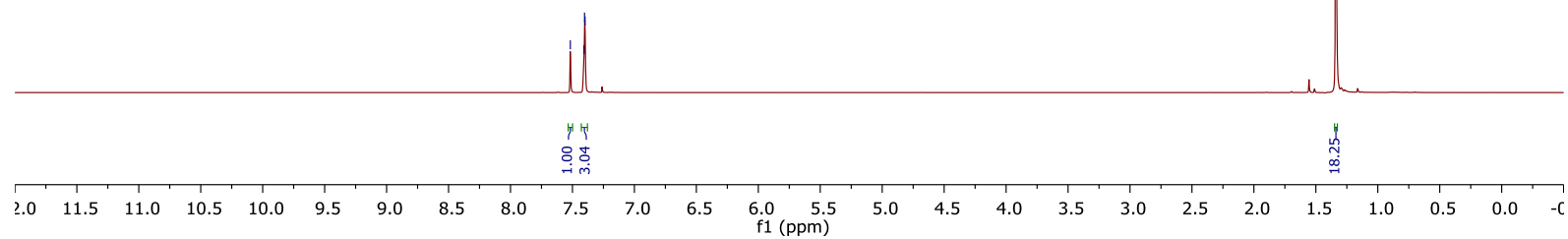<smiles>CC(C)(C)c1cc(C=C(Br)Br)cc(C(C)(C)C)c1</smiles>

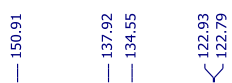

辠

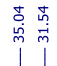

s5, $\mathrm{CDCl}_{3}$

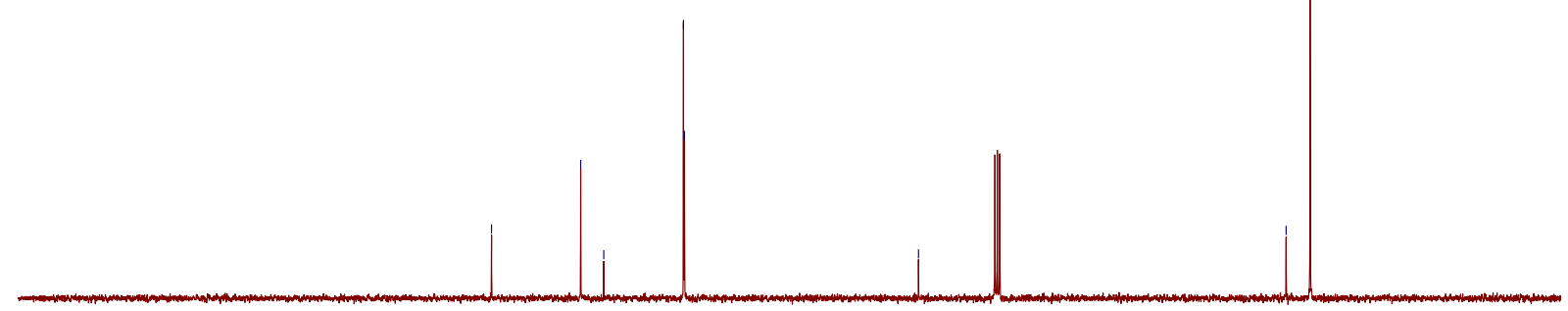

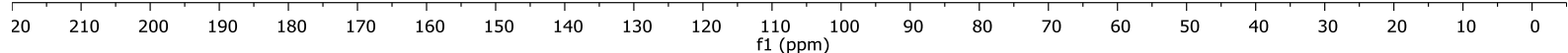




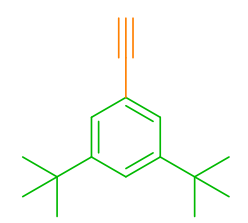

32, $\mathrm{CDCl}_{3}$
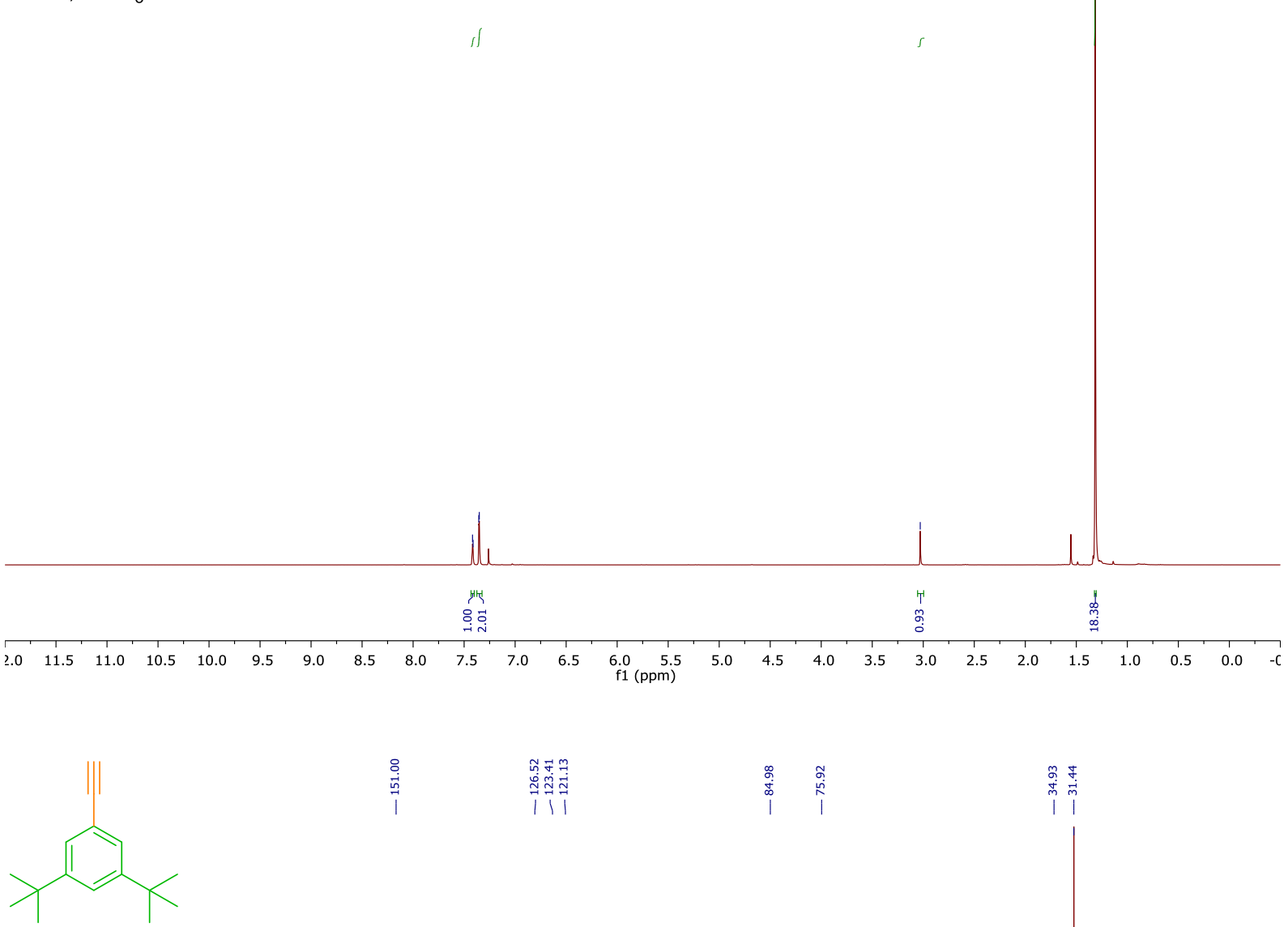

约

$\substack{\infty \\ 0 \\ 0}$
0
0

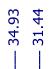

32, $\mathrm{CDCl}_{3}$

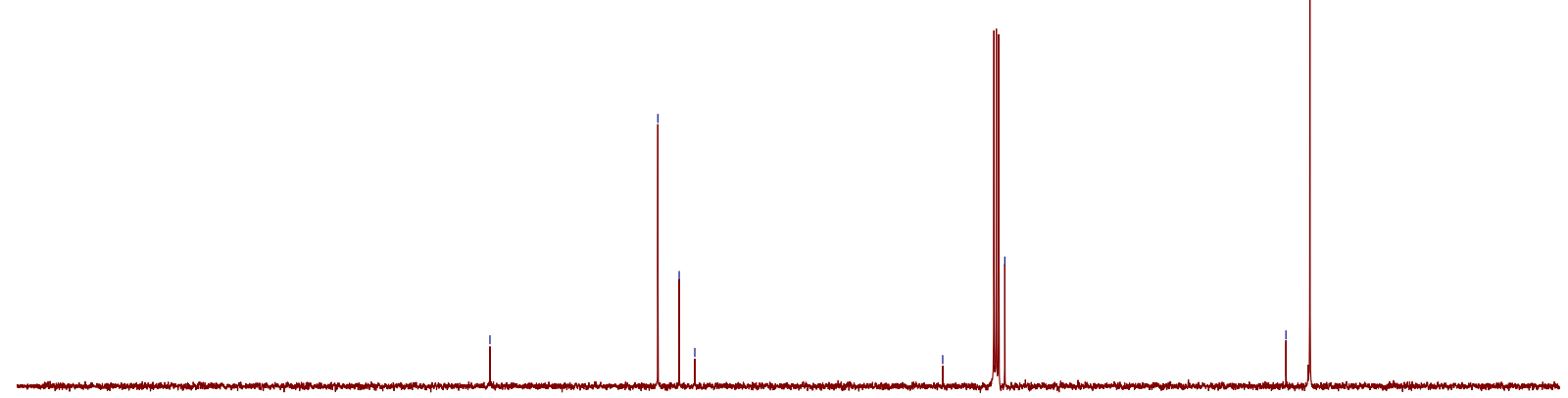

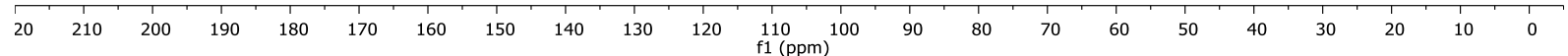



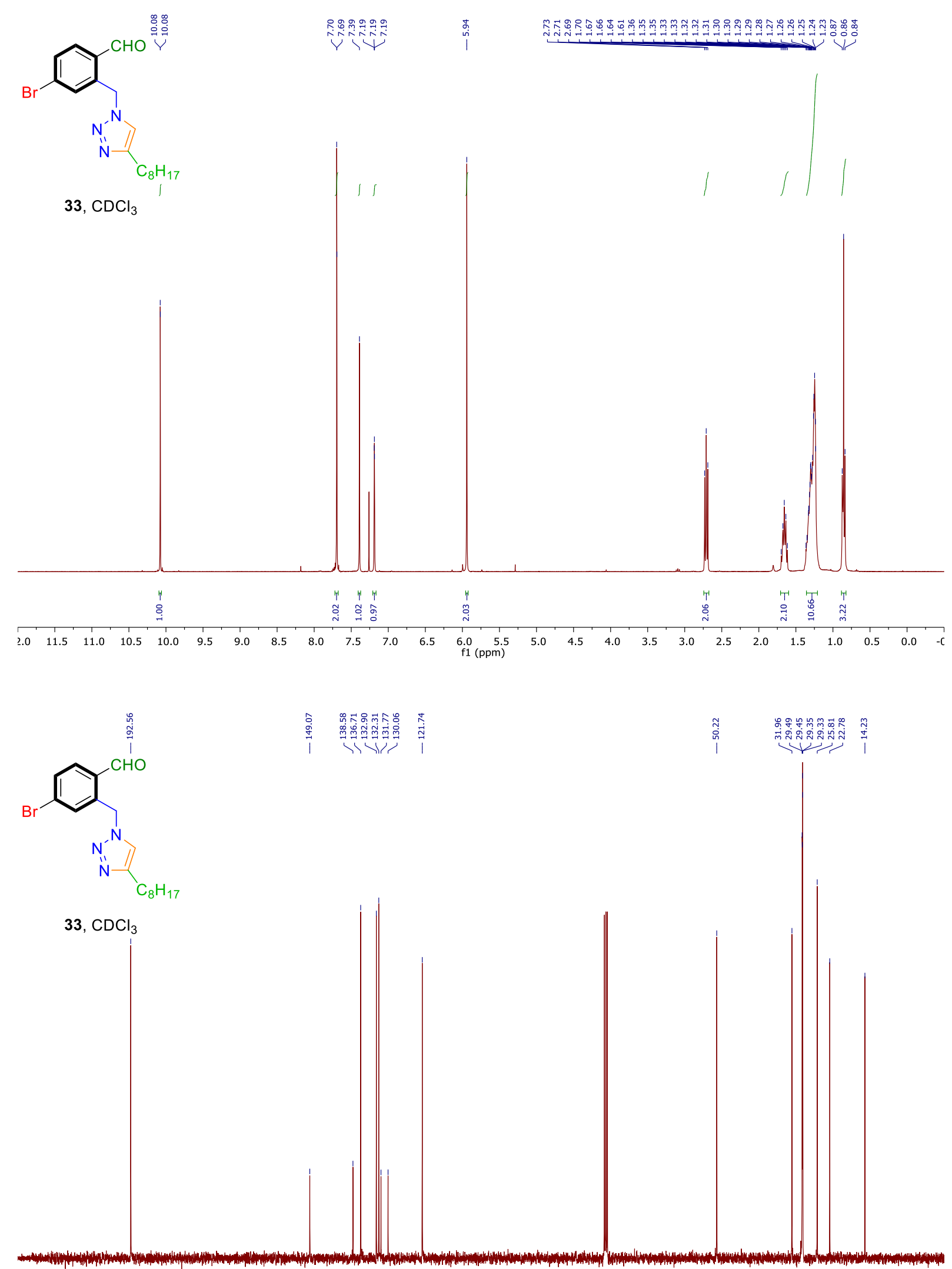

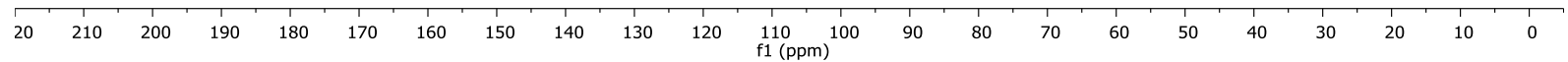




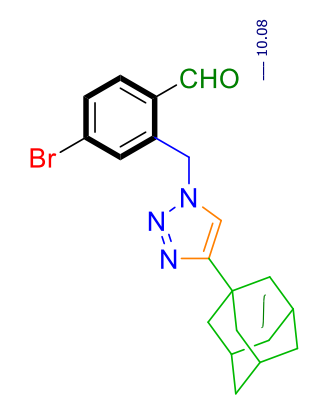

34, $\mathrm{CDCl}_{3}$

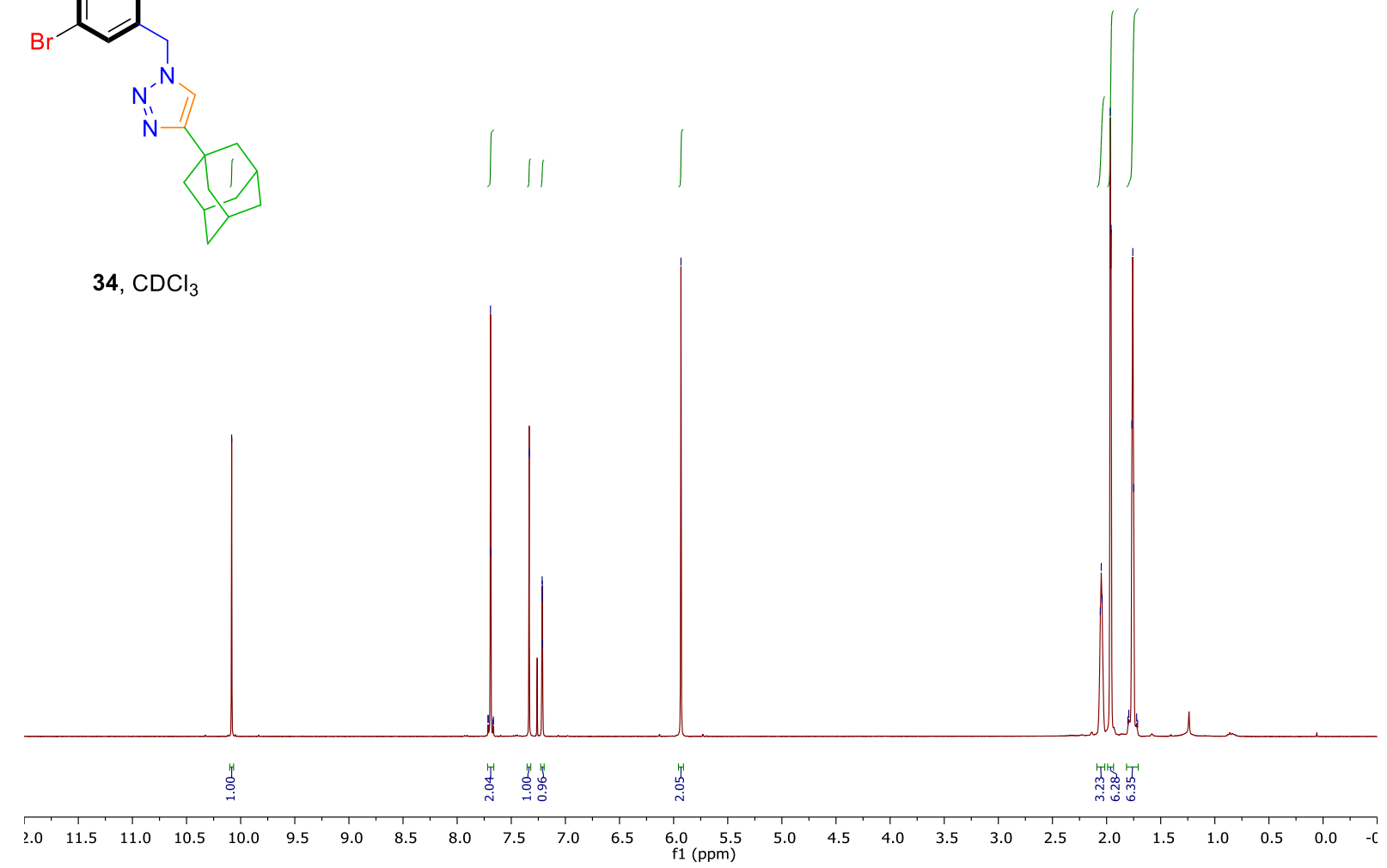

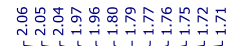

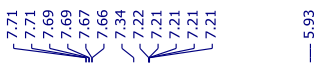
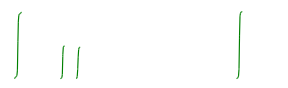

34, $\mathrm{CDCl}_{3}$

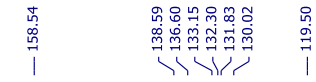
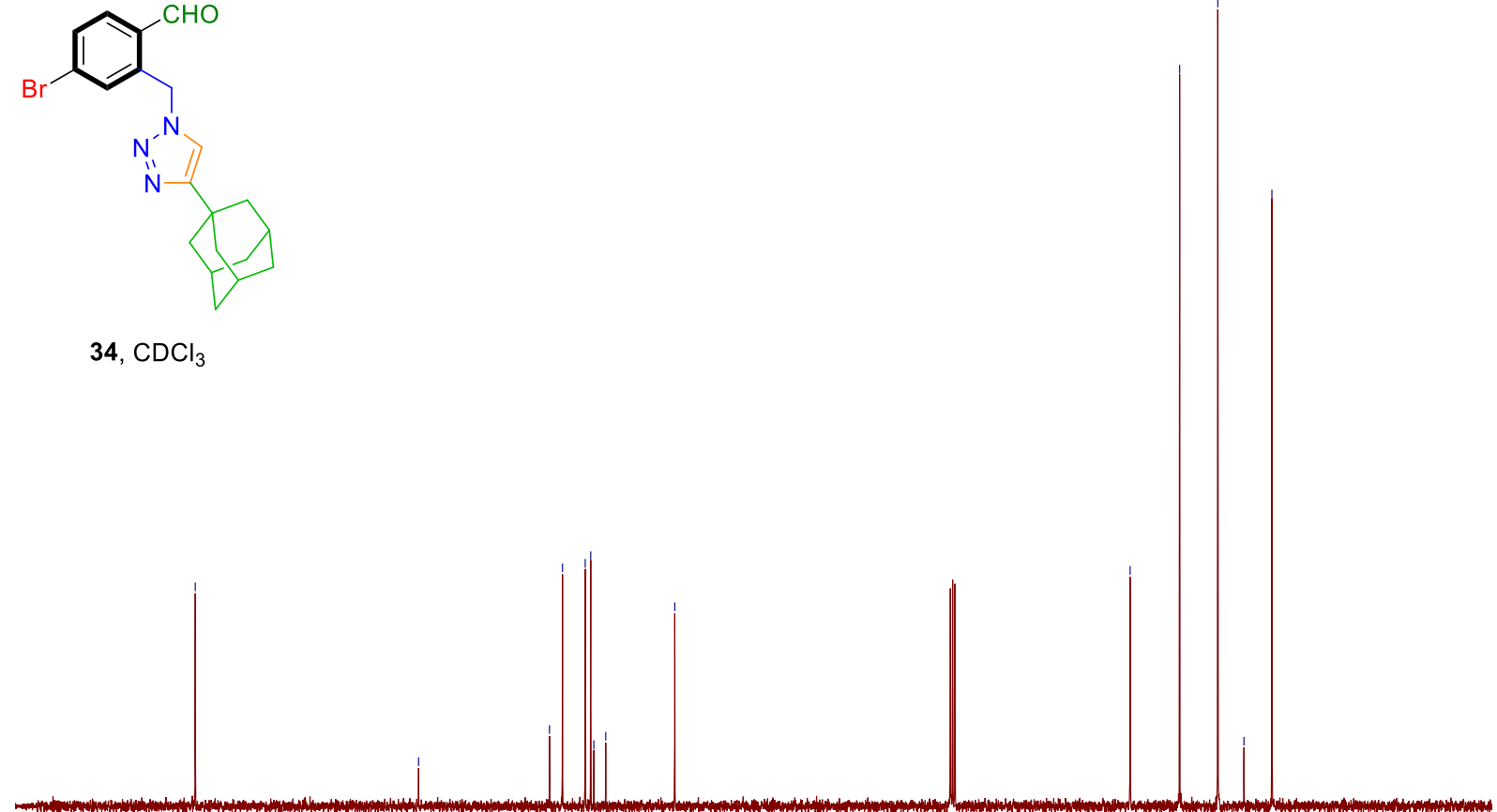

$\begin{array}{lllllllllllllllllllllll}1 & 210 & 200 & 190 & 180 & 170 & 160 & 150 & 140 & 130 & 120 & \underset{\mathrm{f} 1(\mathrm{ppm})}{110} & 100 & 90 & 80 & 70 & 60 & 50 & 40 & 30 & 20 & 10 & 0\end{array}$ 


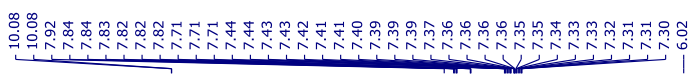
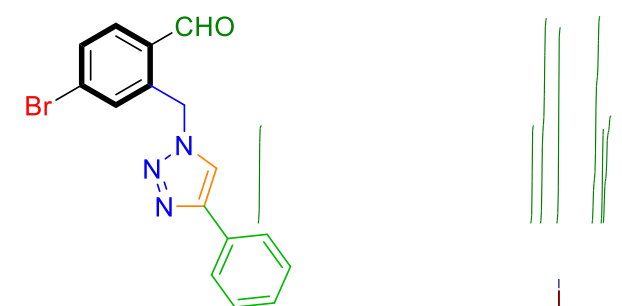

35, $\mathrm{CDCl}_{3}$
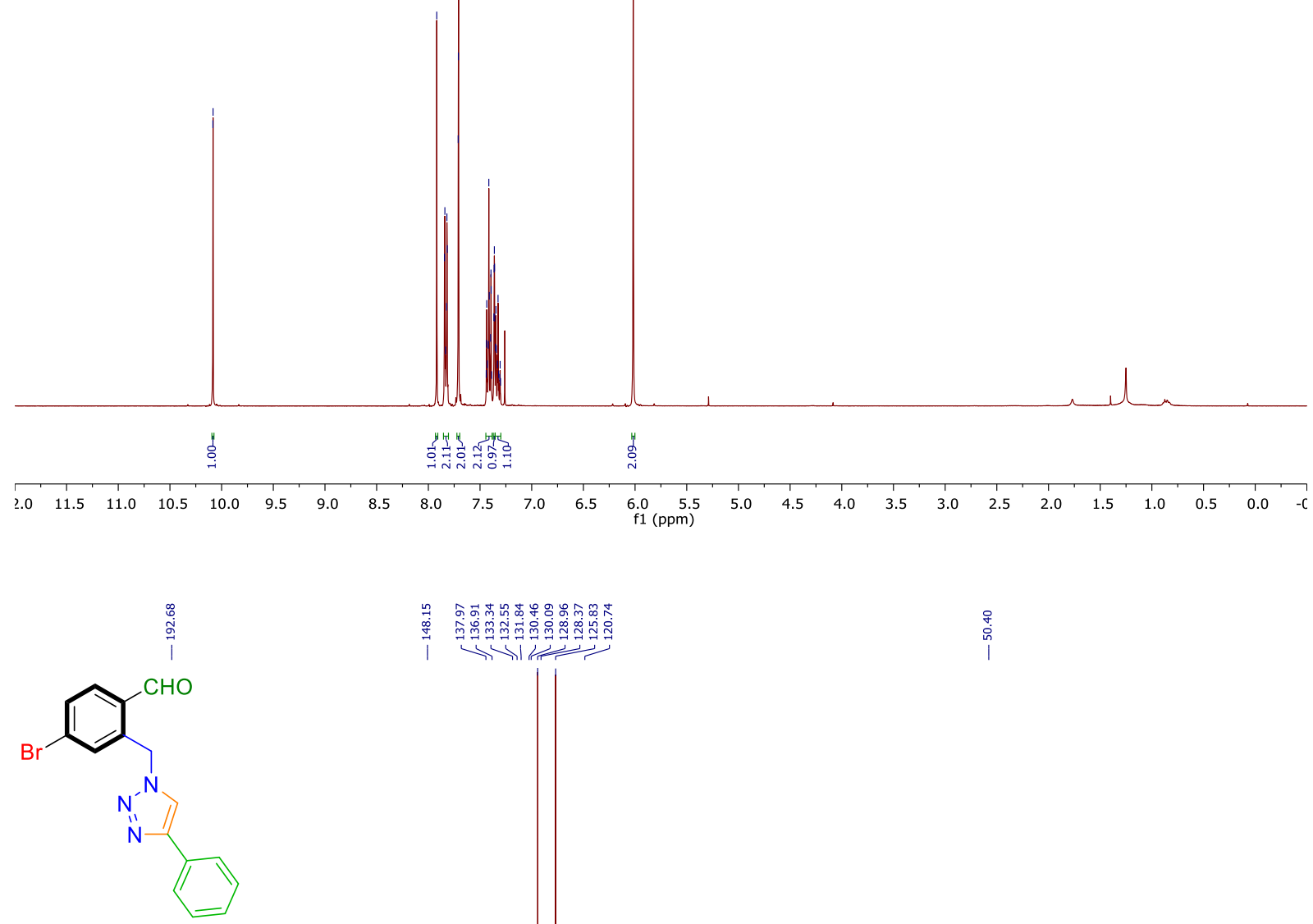

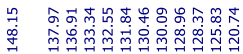

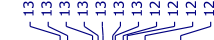

35, $\mathrm{CDCl}_{3}$

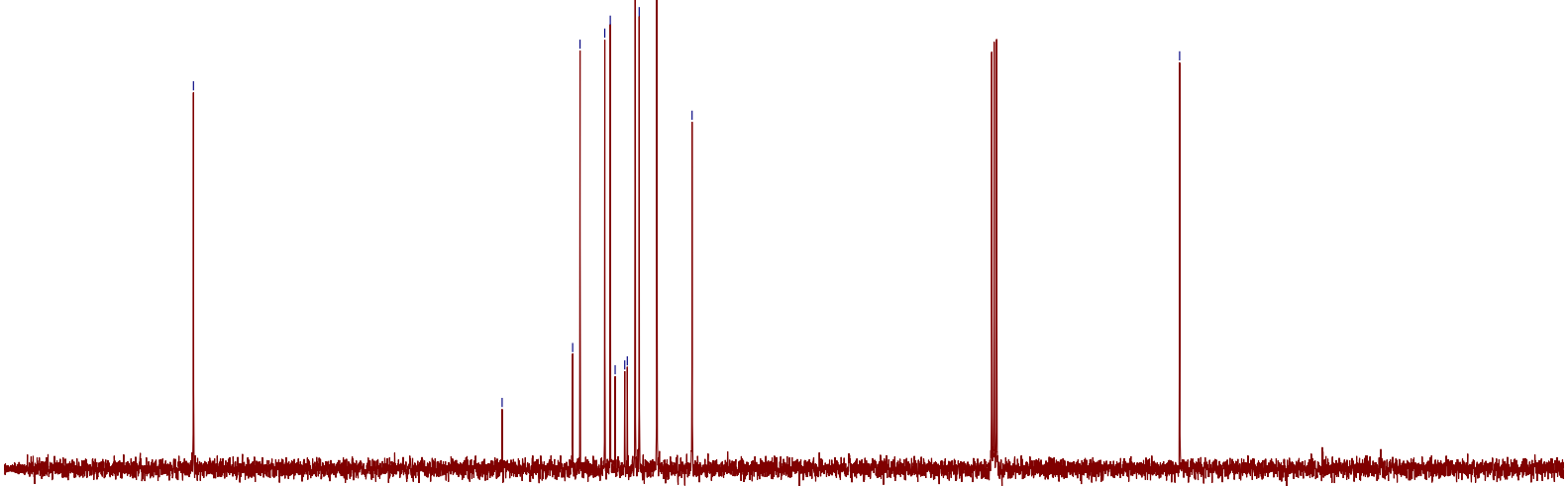

$\begin{array}{lllllllllllllllllllllllll}10 & 210 & 200 & 190 & 180 & 170 & 160 & 150 & 140 & 130 & 120 & \underset{f 1}{110}(\mathrm{ppm}) & 100 & 90 & 80 & 70 & 60 & 50 & 40 & 30 & 20 & 10 & 0\end{array}$ 


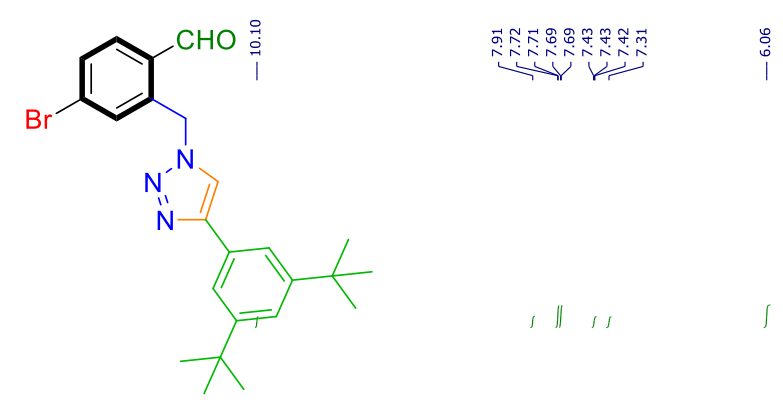

36, $\mathrm{CDCl}_{3}$
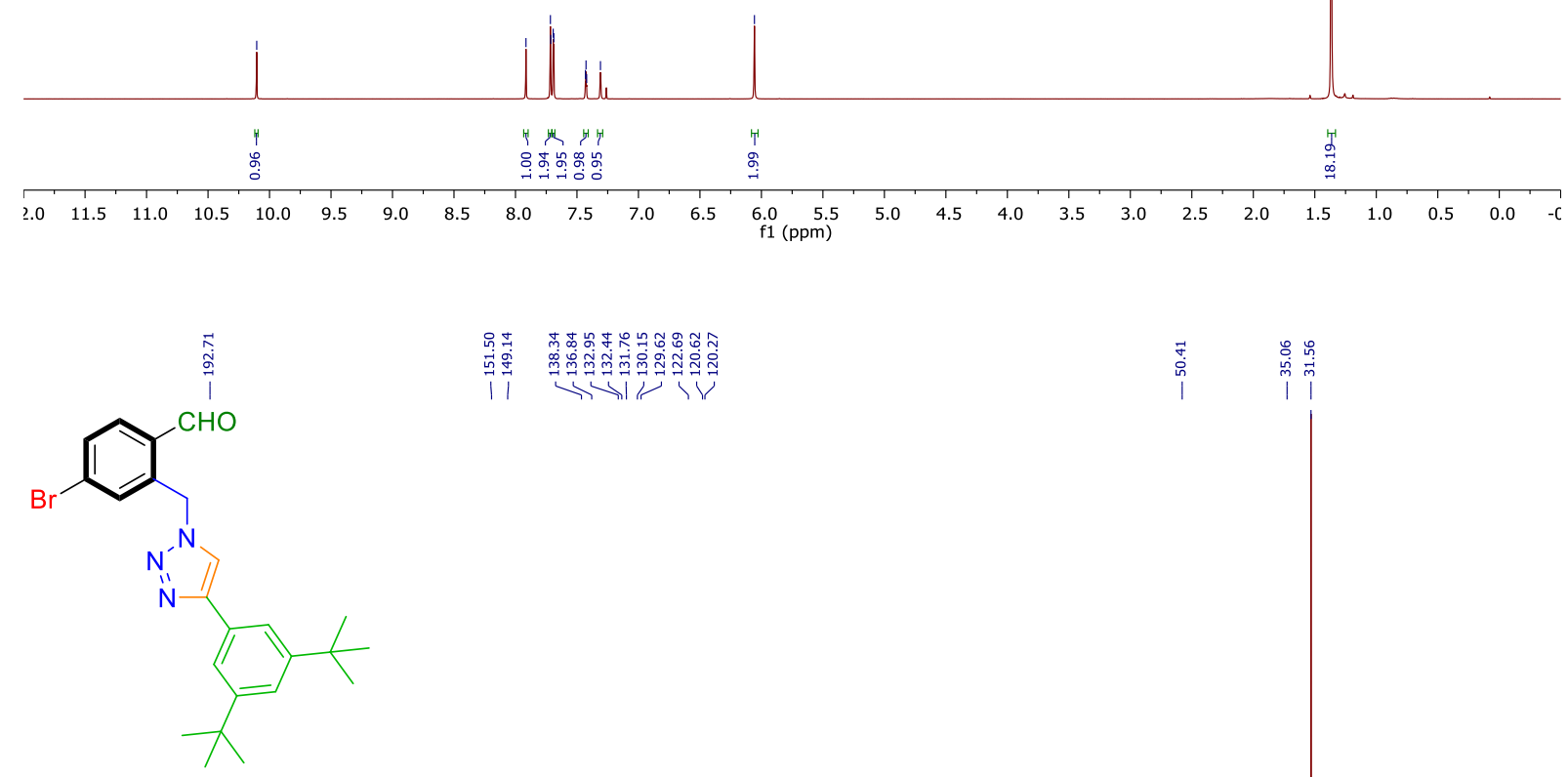

36, $\mathrm{CDCl}_{3}$

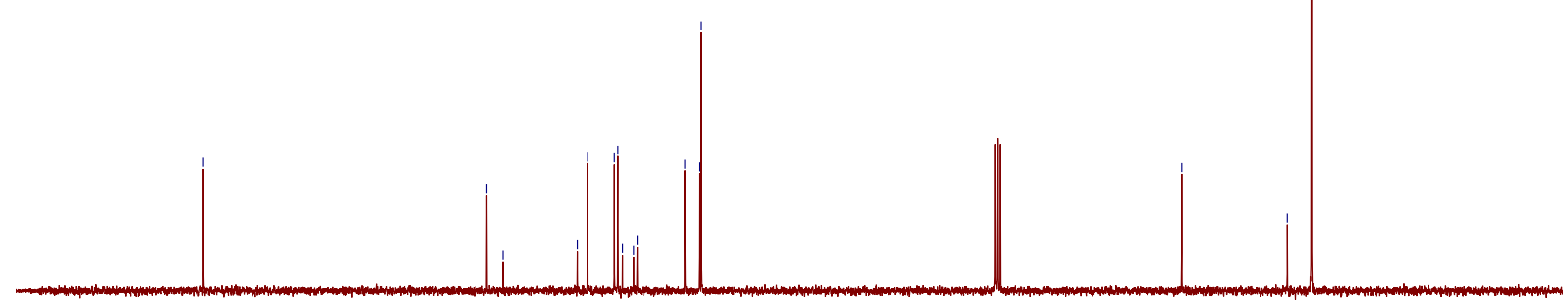

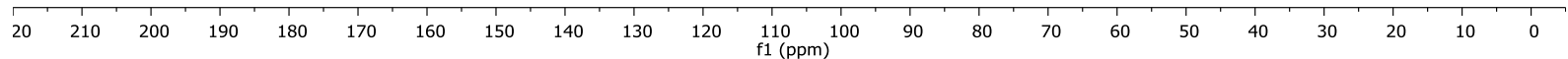




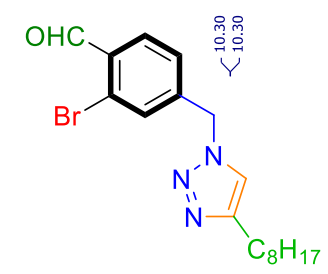

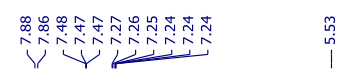

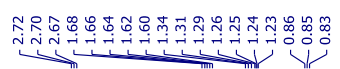

37, $\mathrm{CDCl}_{3}$
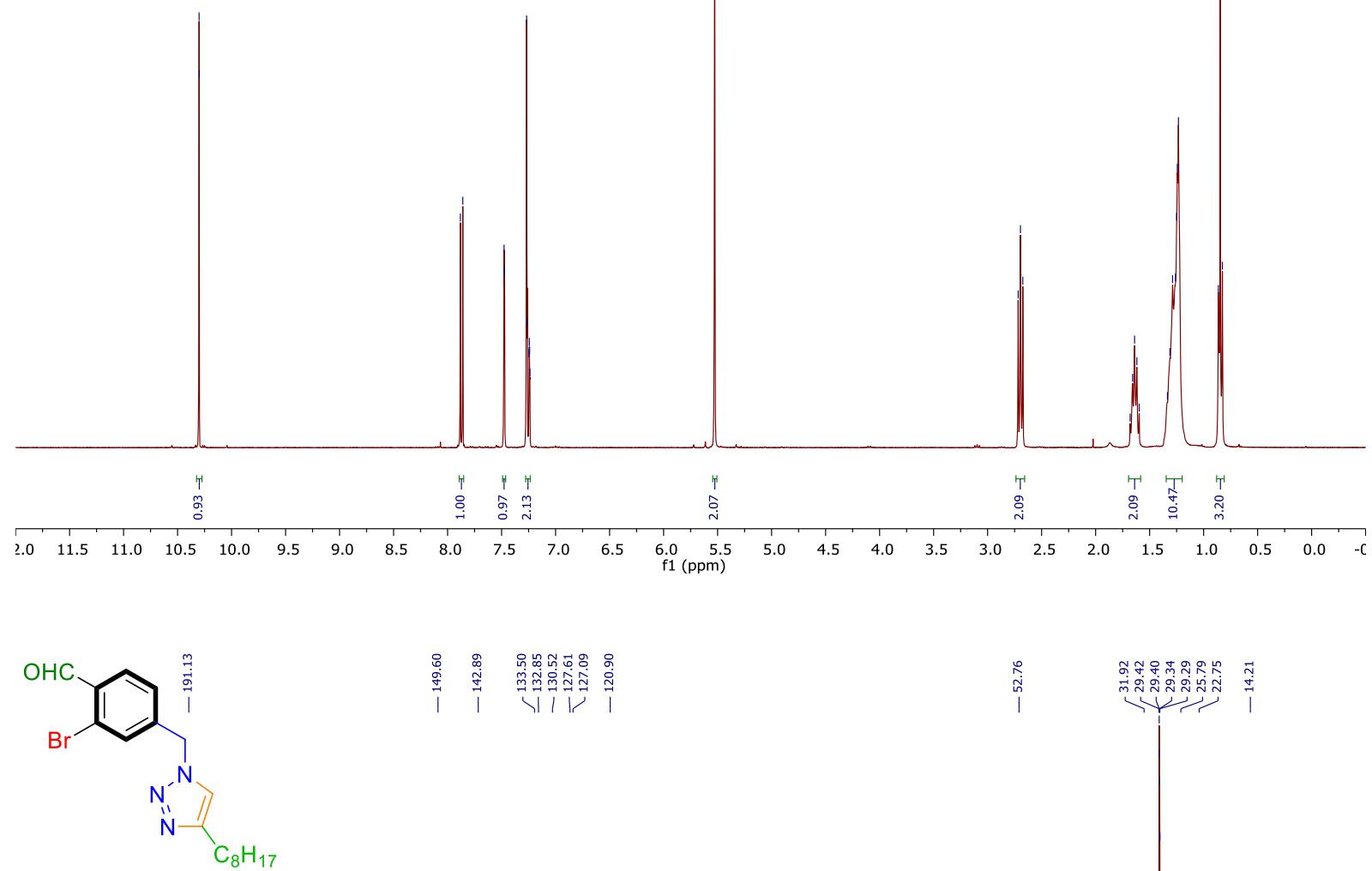

37, $\mathrm{CDCl}_{3}$

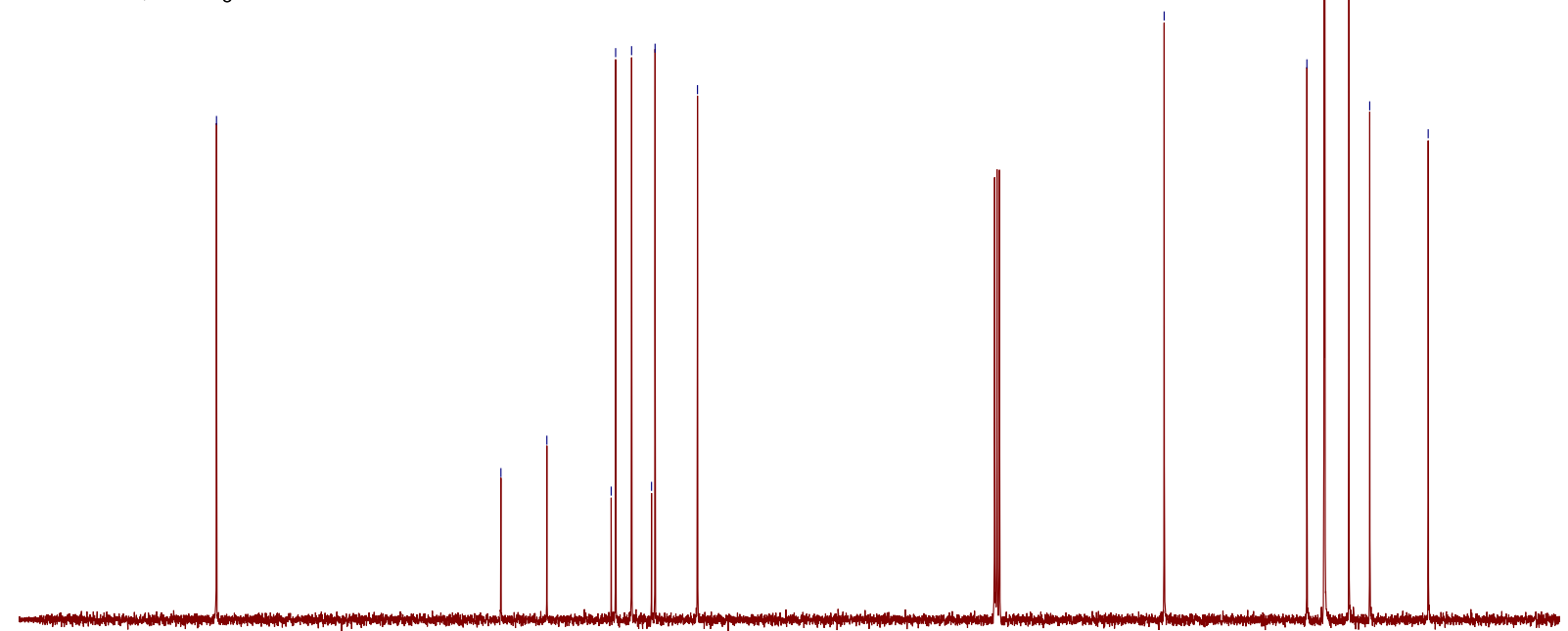

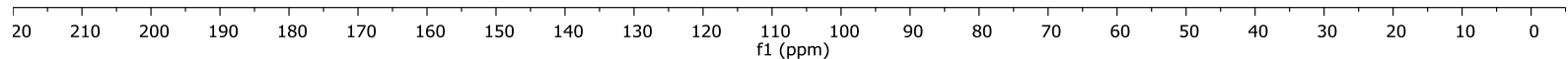



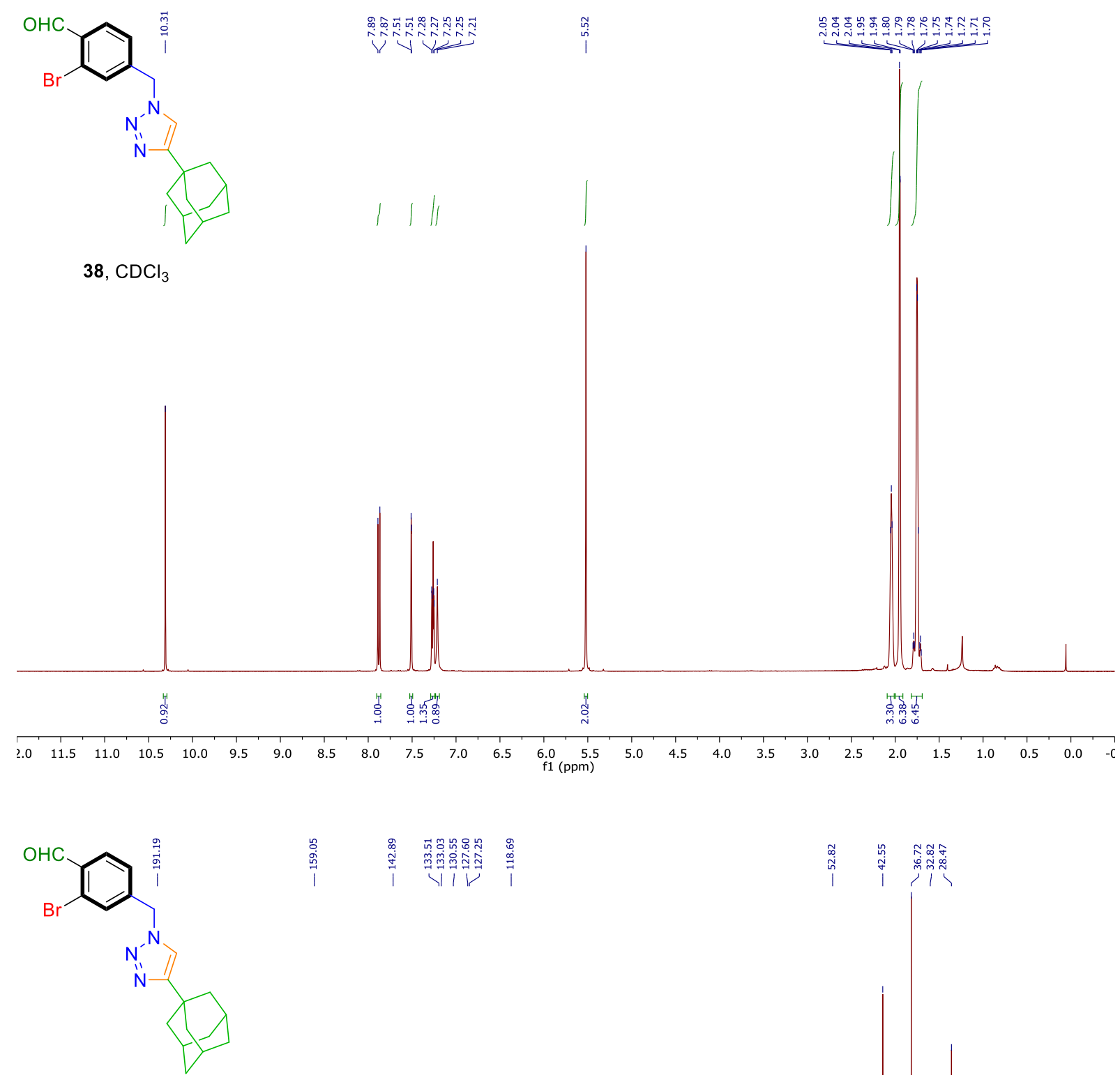

38, $\mathrm{CDCl}_{3}$

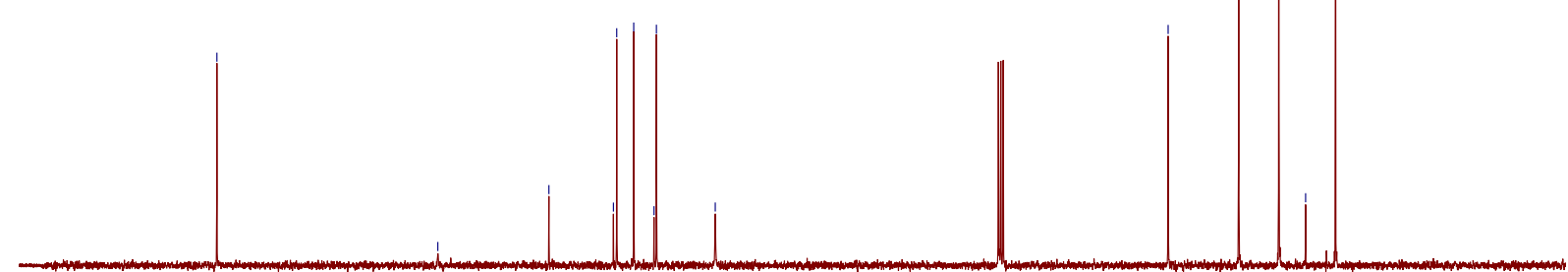

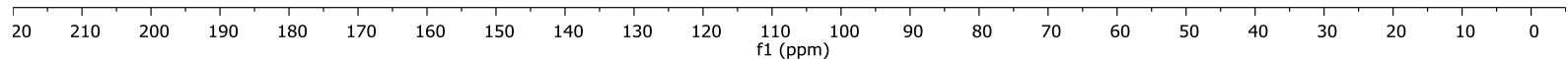




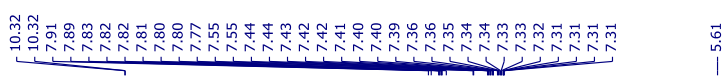
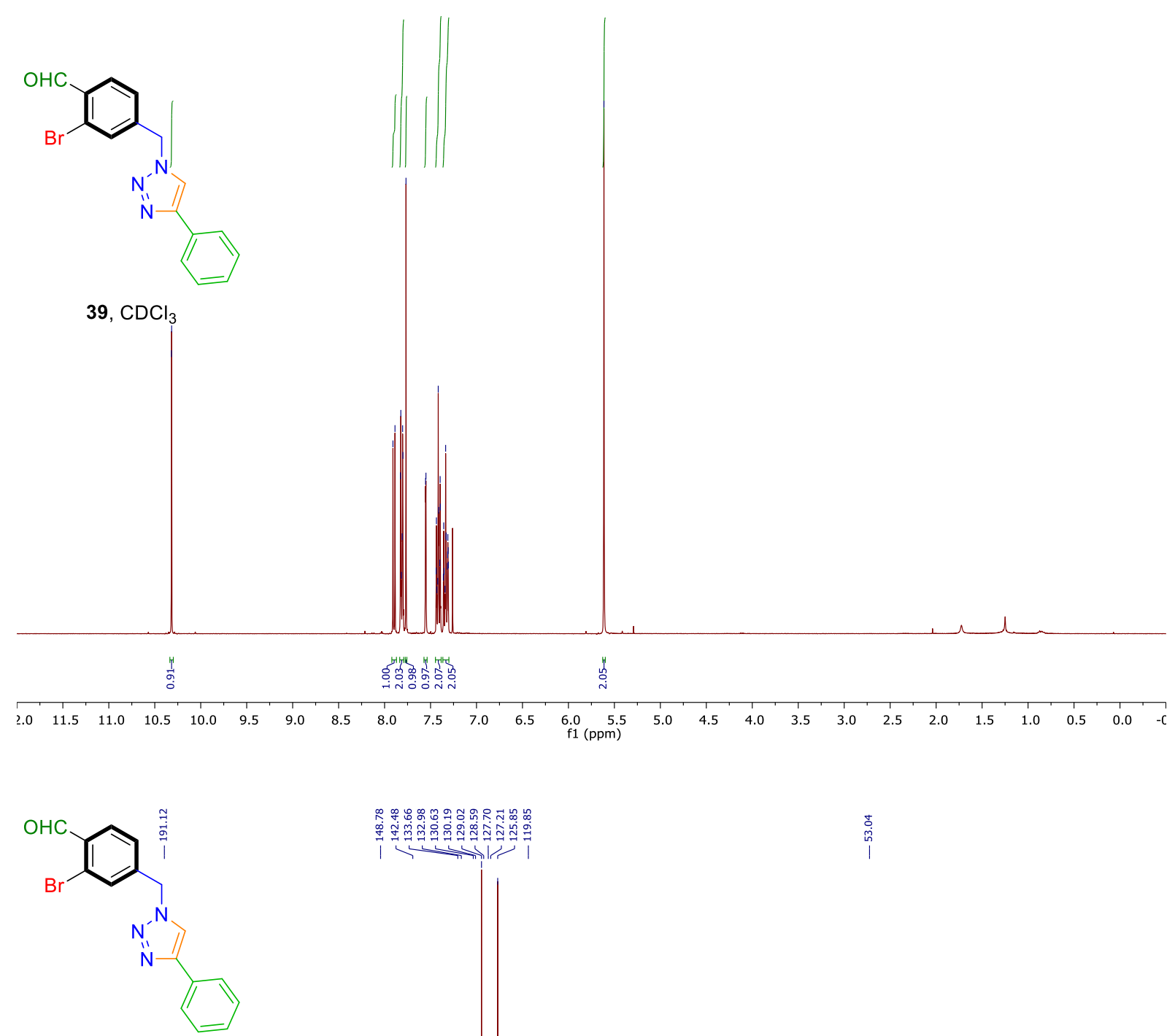

39, $\mathrm{CDCl}_{3}$

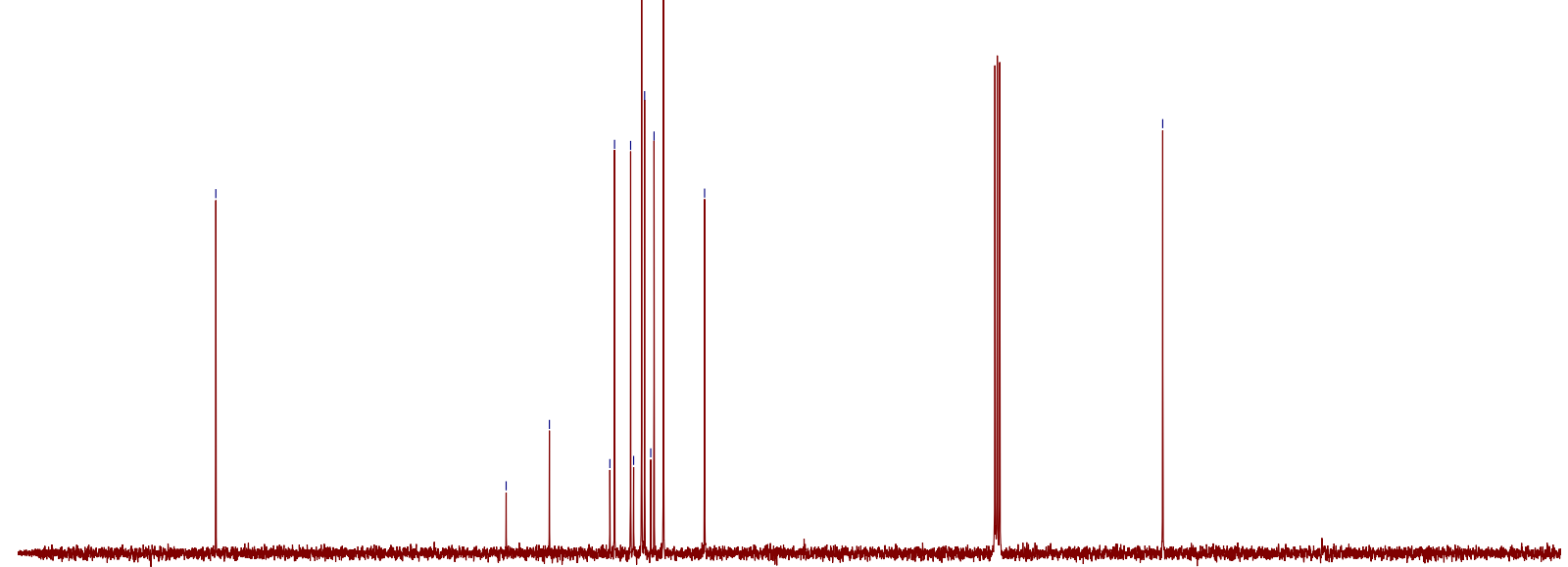

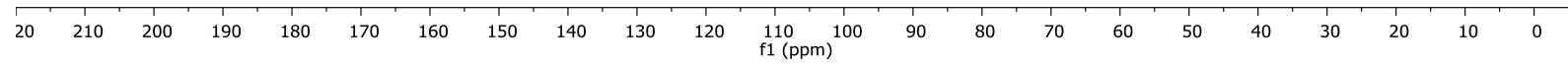



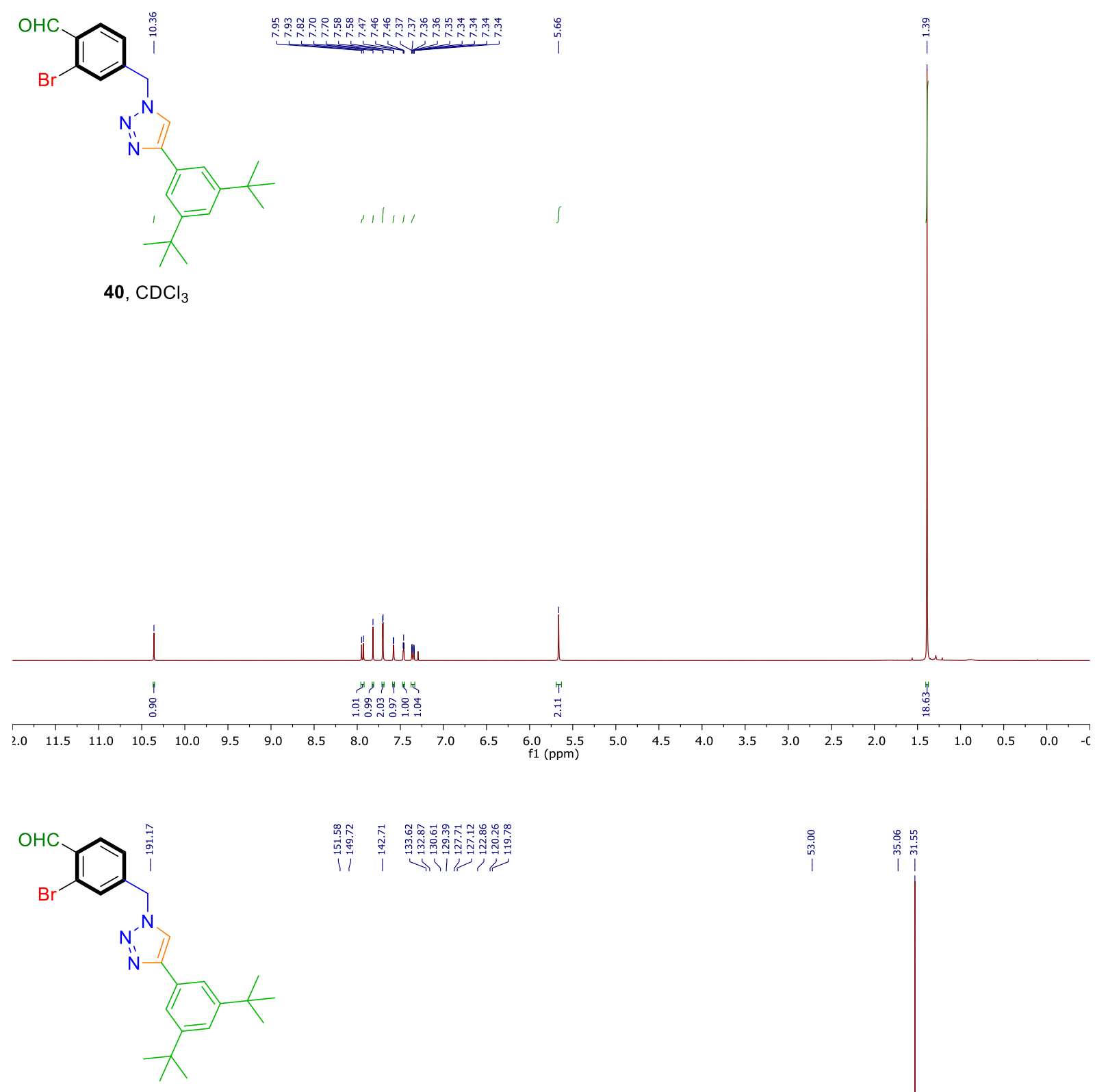

40, $\mathrm{CDCl}_{3}$

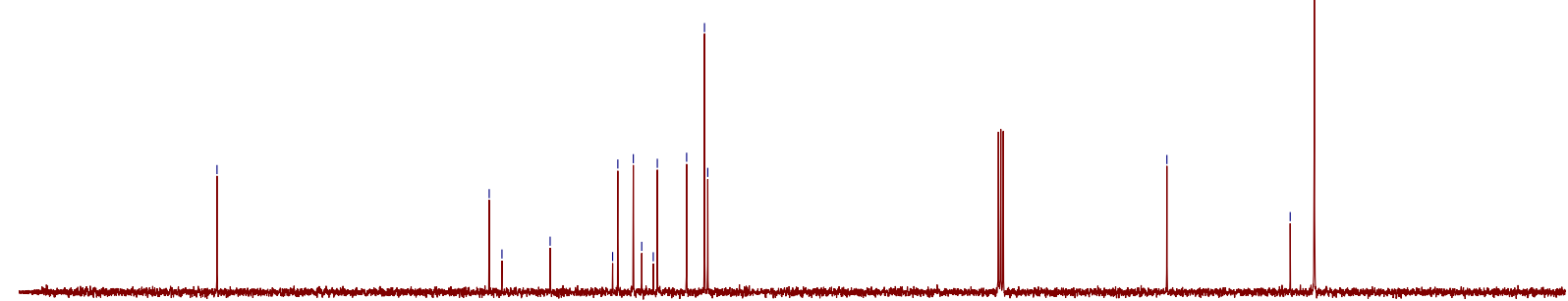

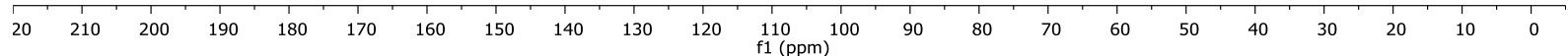



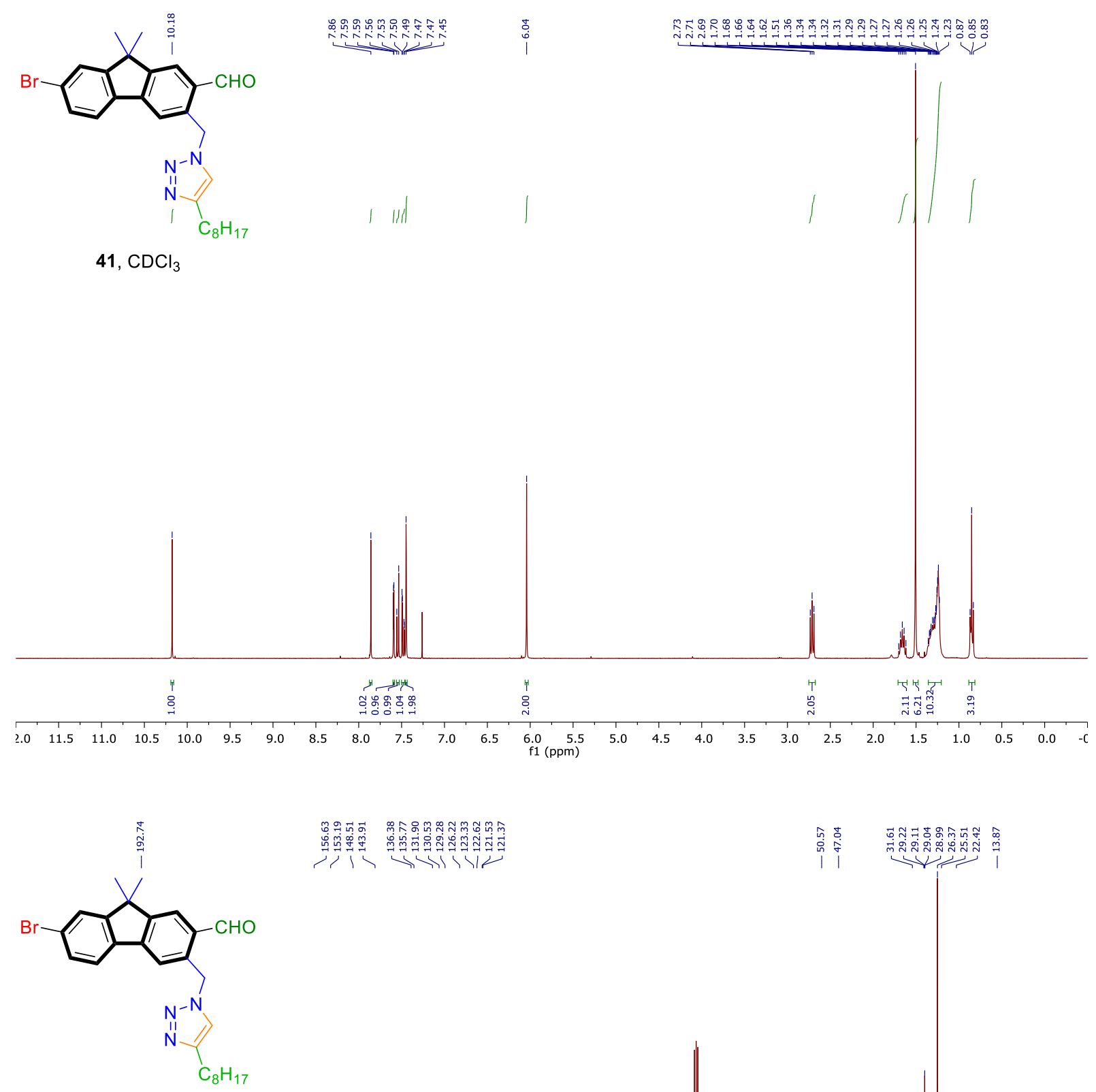

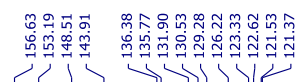

至

। I

41, $\mathrm{CDCl}_{3}$

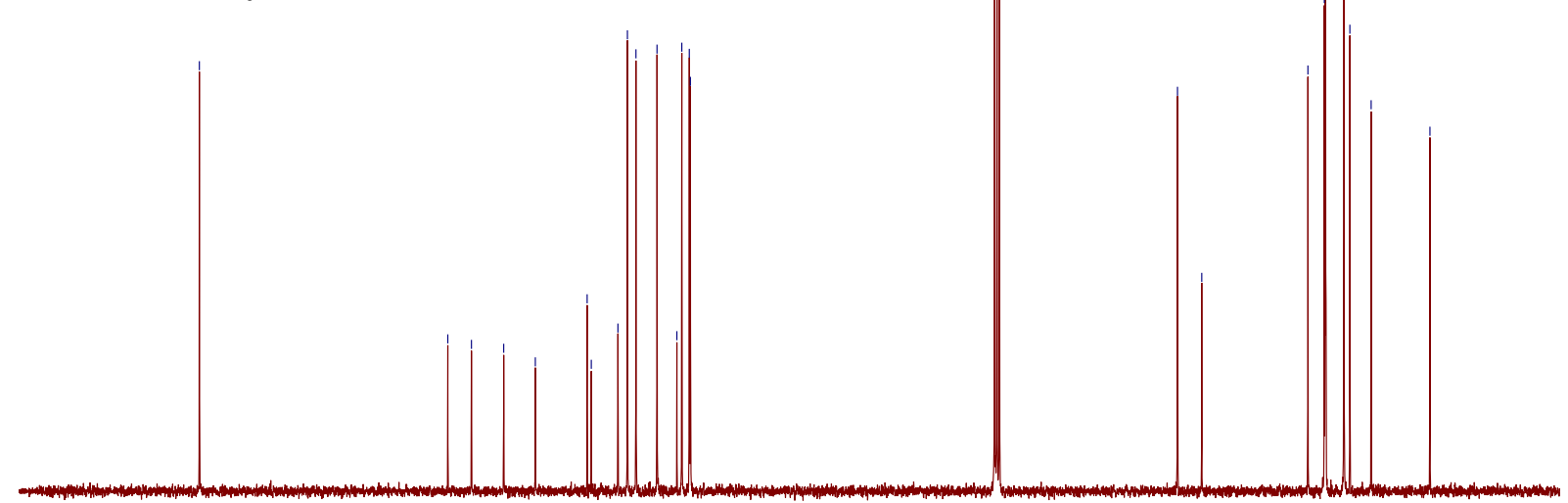

$\begin{array}{lllllllllllllllllllllll}210 & 200 & 190 & 180 & 170 & 160 & 150 & 140 & 130 & 120 & \begin{array}{l}110 \\ \mathrm{f} 1(\mathrm{ppm})\end{array} & 100 & 90 & 80 & 70 & 60 & 50 & 40 & 30 & 20 & 10 & 0\end{array}$ 

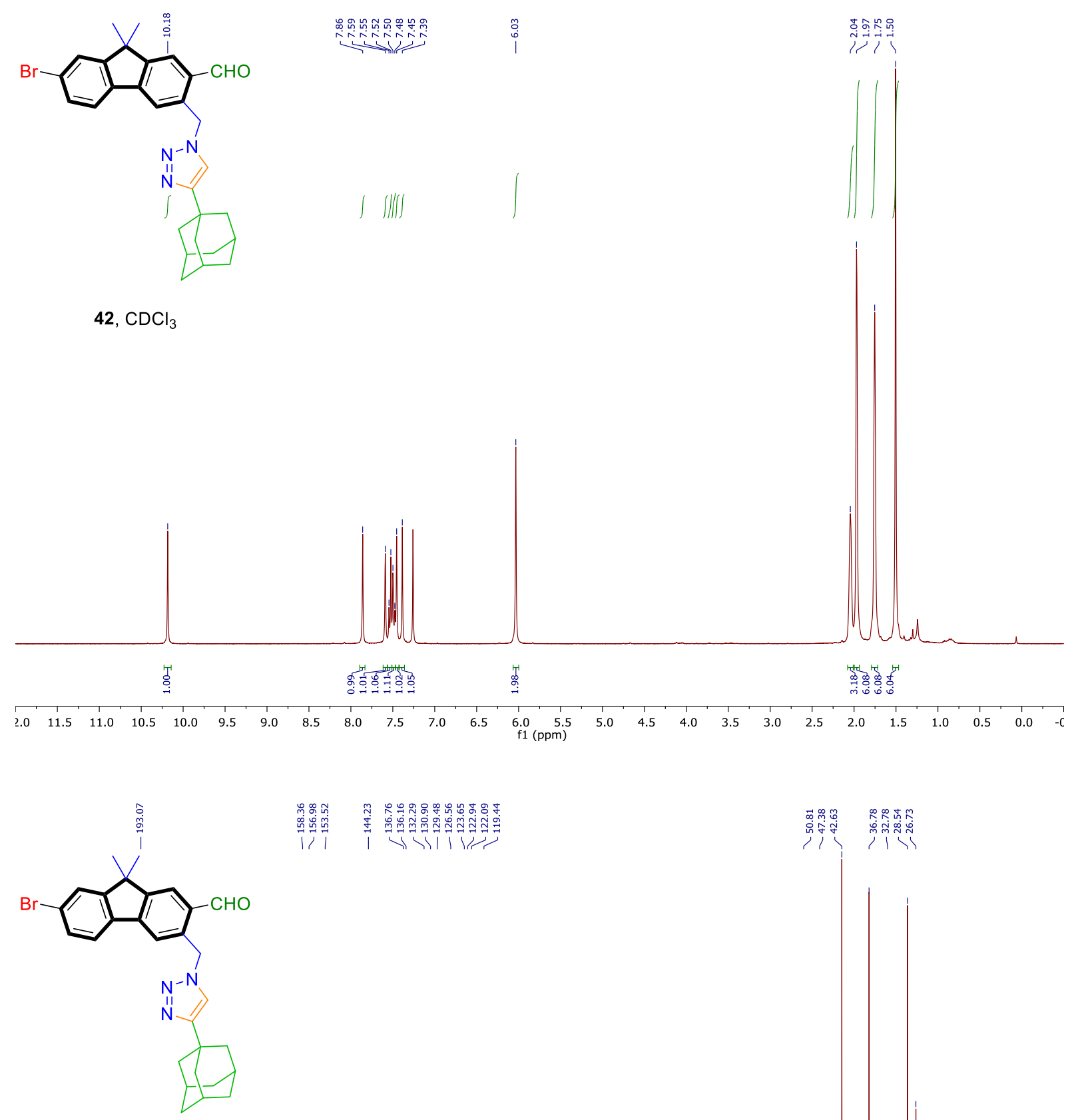

42, $\mathrm{CDCl}_{3}$

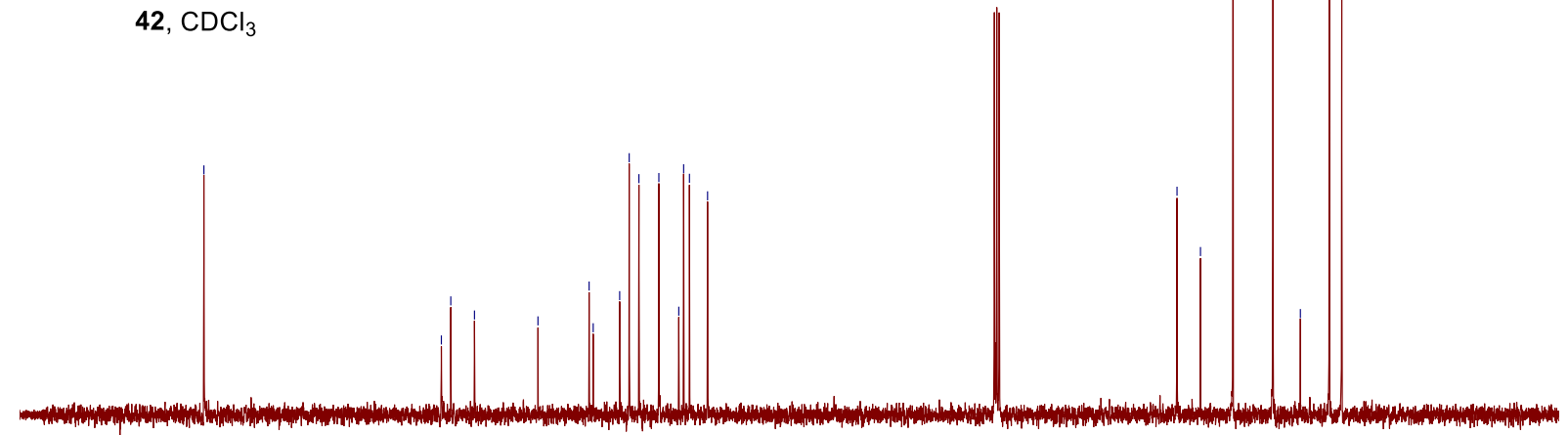

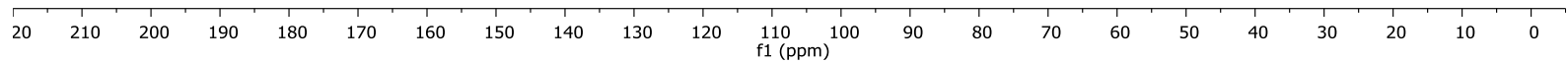




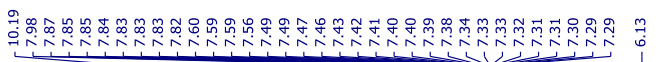
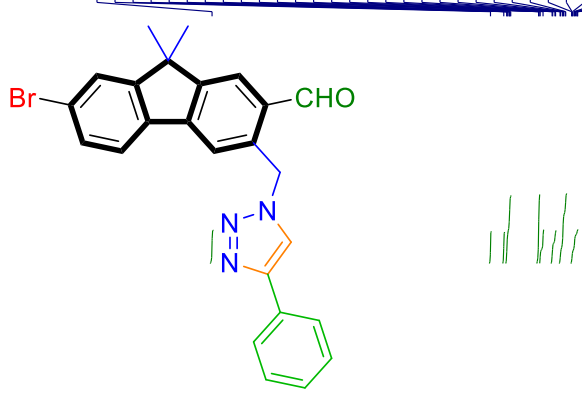

43, $\mathrm{CDCl}_{3}$
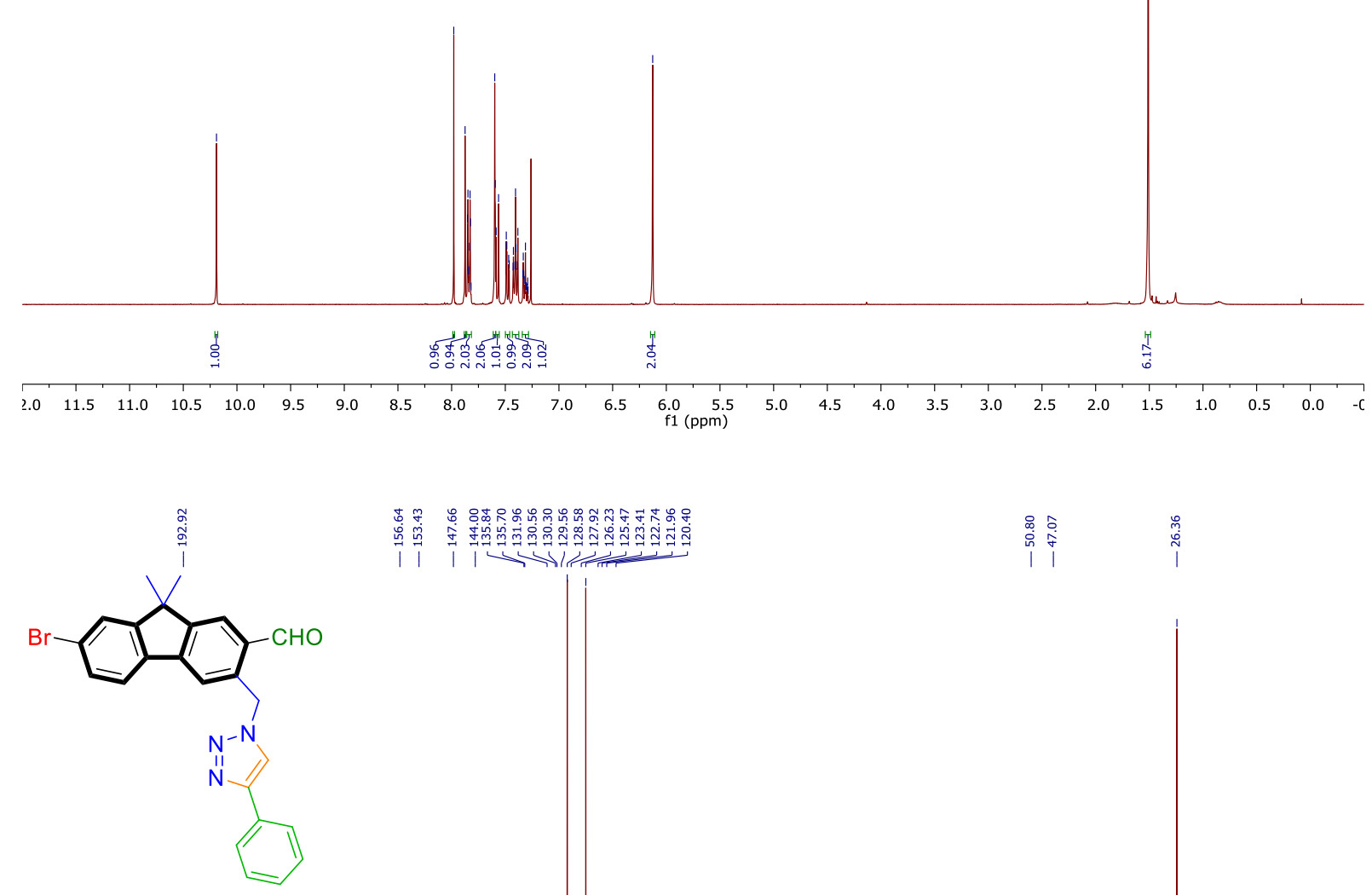

43, $\mathrm{CDCl}_{3}$

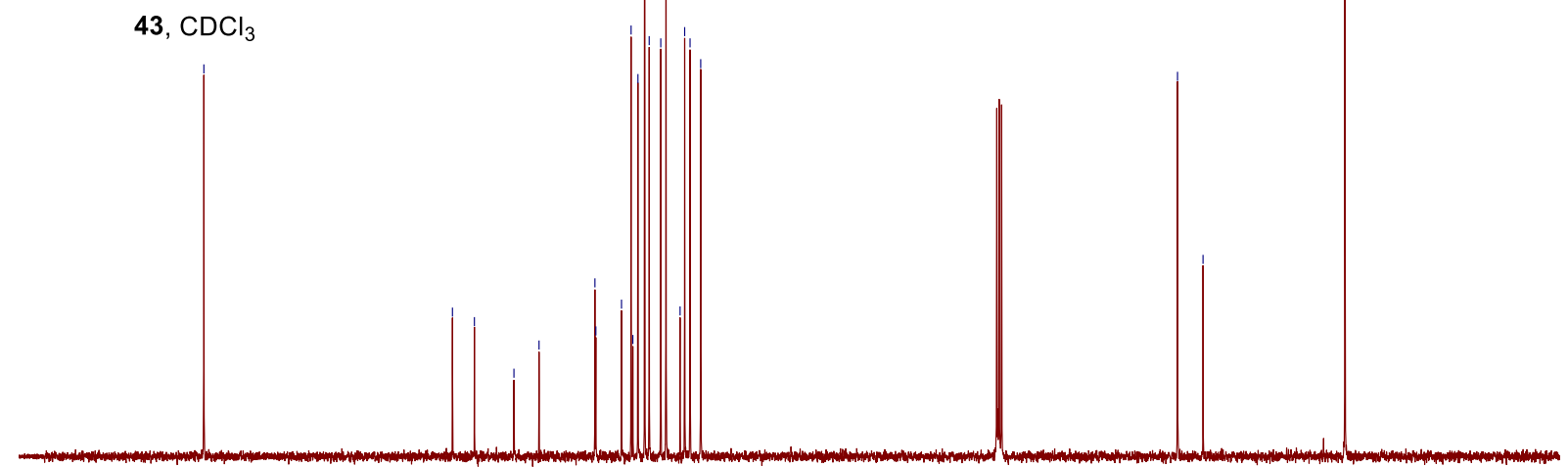

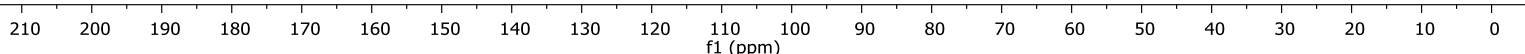



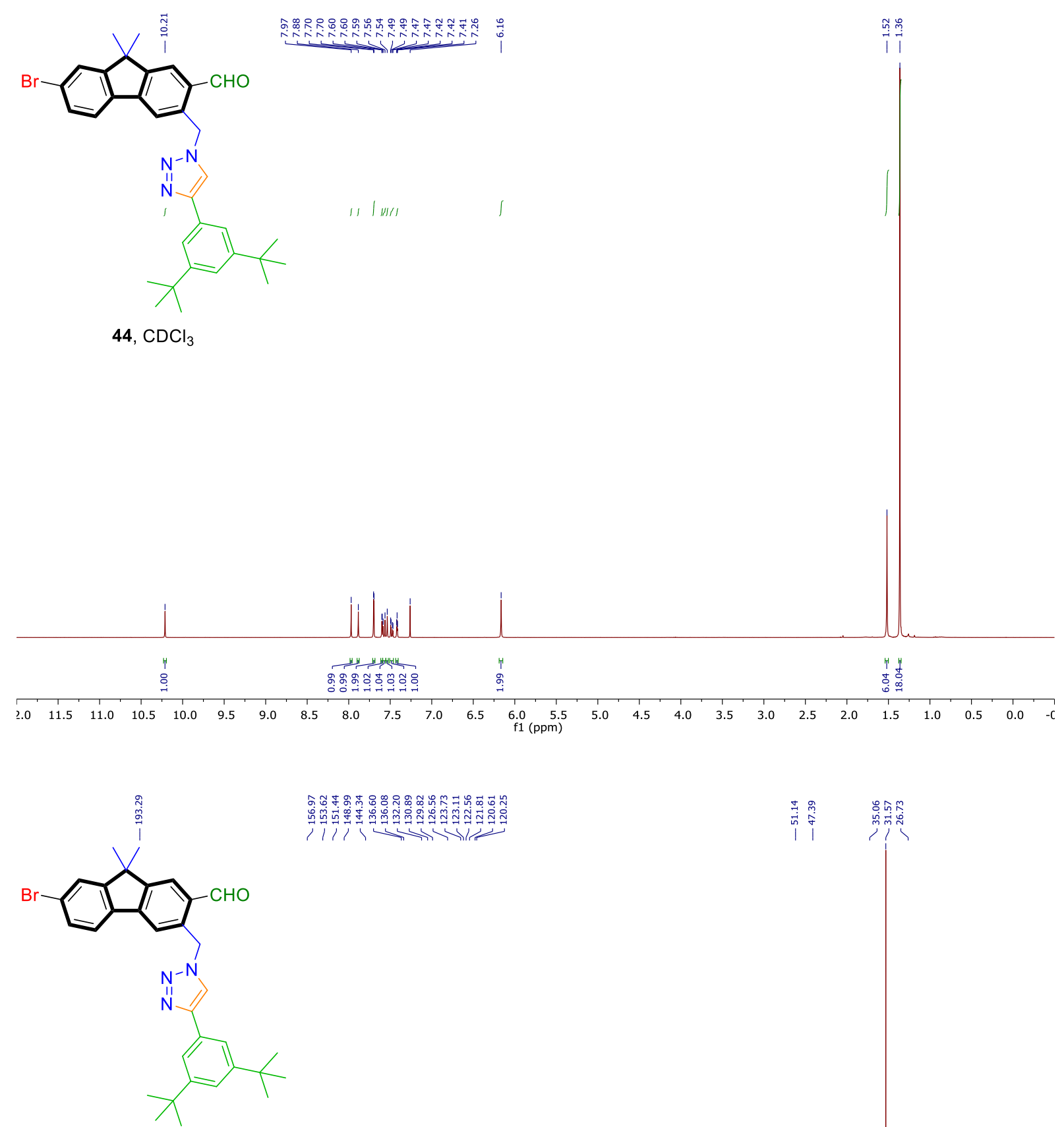

2
0

44, $\mathrm{CDCl}_{3}$

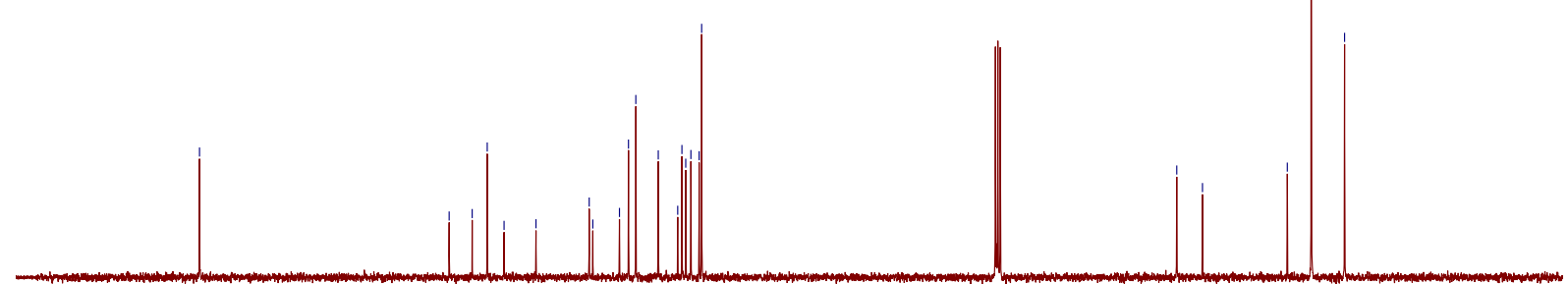

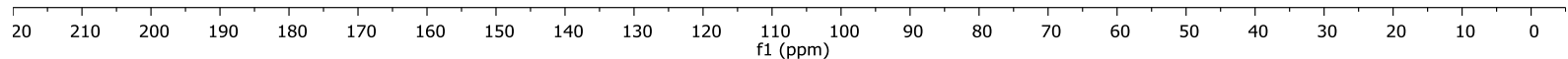



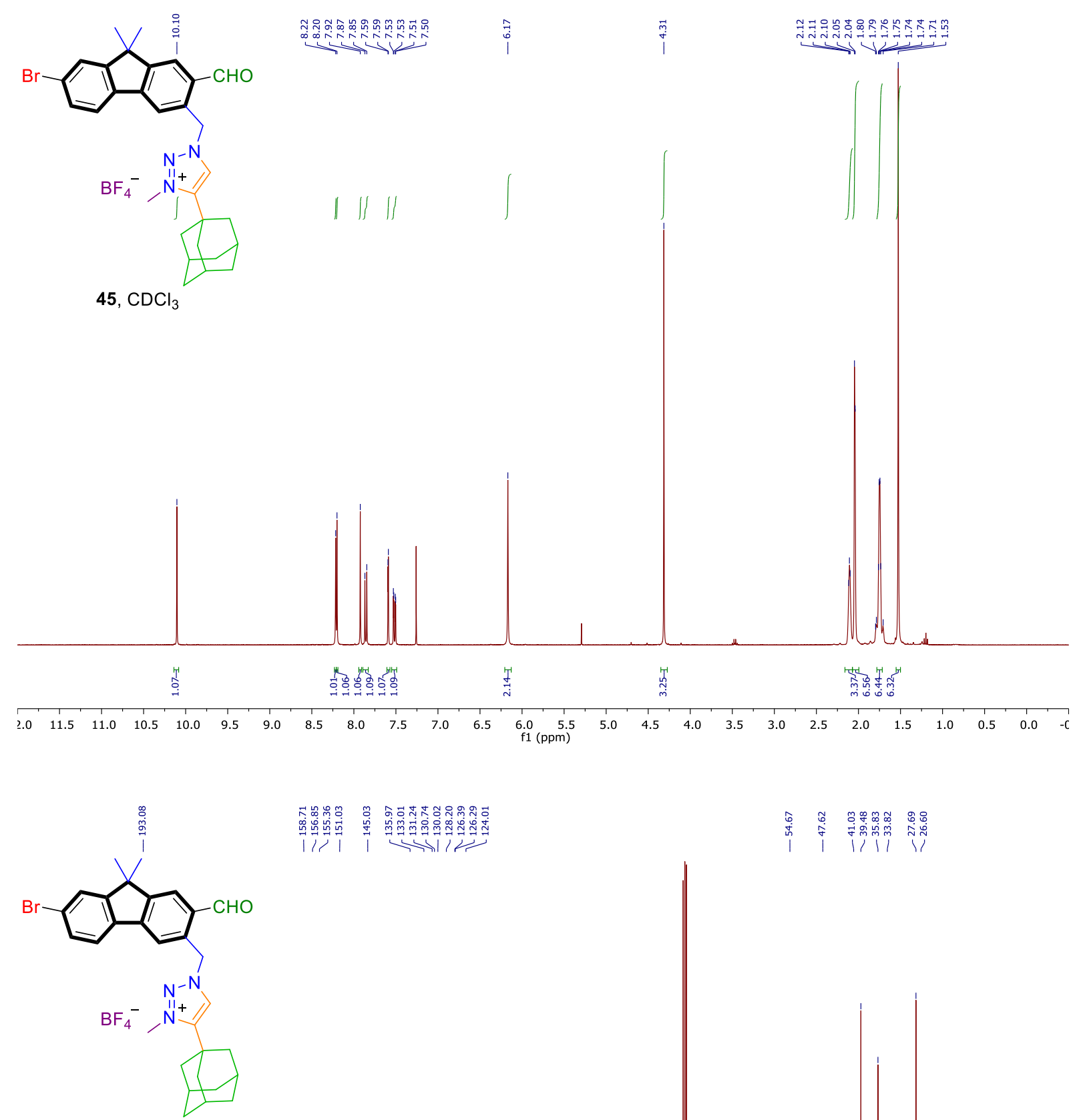

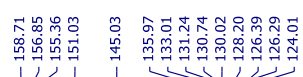

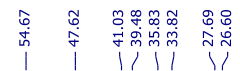

45, $\mathrm{CDCl}_{3}$

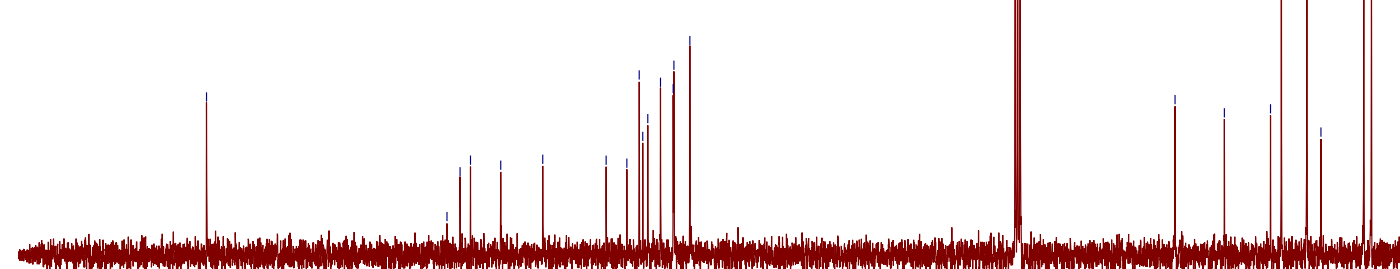

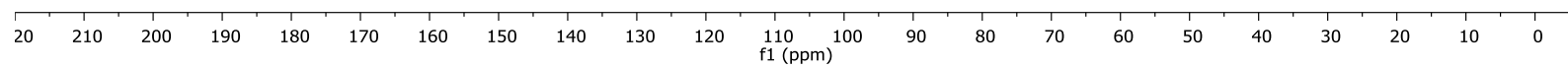


Other files

Graphical Abstract.TIF (1.17 MiB)

view on ChemRxiv - download file 